CARLOS NEHEMY MARMO

\title{
BIFURCAÇÕES EM PLLS DE TERCEIRA ORDEM EM REDES OWMS
}

\author{
Tese apresentada à Escola \\ Politécnica da Universidade de \\ São Paulo para obtenção do \\ Título de Doutor em Engenharia.
}

São Paulo

2008 
CARLOS NEHEMY MARMO

\section{BIFURCAÇÕES EM PLLS DE TERCEIRA ORDEM EM REDES OWMS}

Tese apresentada à Escola

Politécnica da Universidade de São Paulo para obtenção do Título de Doutor em Engenharia.

Área de Concentração:

Engenharia Elétrica

Orientador: José Roberto Castilho Piqueira

São Paulo 
Marmo, Carlos Nehemy

Bifurcações em PLLs de terceira ordem em redes OWMS / C. N. Marmo. -- São Paulo, 2008.

p.

Tese (Doutorado) - Escola Politécnica da Universidade de São Paulo. Departamento de Engenharia de Telecomunicações e Controle.

1.Equações diferenciais 2.Sistemas dinâmicos

I.Universidade

de São Paulo. Escola Politécnica. Departamento de Engenharia de Telecomunicações e Controle Il.t. 


\section{AGRADECIMENTOS}

Em primeiro lugar, esta pesquisa só foi possível graças ao total suporte do EPUSP (Escola Politécnica da Universidade de São Paulo), dos seus professores e pesquisadores e do apoio financeiro da CAPES (Coordenação de Aperfeiçoamento de Pessoal de Nível Superior).

Em segundo, gostaria de deixar aqui, os meus mais sinceros agradecimentos às seguintes pessoas, que contribuíram de forma mais decisiva para o doutorado que encerro com esta tese:

- Aos meus amigos Fernando Orsatti, Solange Kusaba, Danilo Pinseta e Élcio M. Silveira que muito me ajudaram de todas as formas que puderam, principalmente após o falecimento de papai, logo no início do doutorado. Tive sorte de conhecer e compartilhar muitos momentos com pessoas tão inteligentes e bondosas.

- À minha esposa, Elizete Perroni Marmo... É impossível de se expressar por palavras a gratidão por tudo o que faz por mim, todos os dias, todos os minutos. Ela é uma jóia rara. Eu te amo, Li.

- À minha mãe, Maria A. S. N. Marmo, que me ensinou quase tudo o que sei. Se tenho qualidades, certamente devo a ela.

- Ao meu primo, Alexandre Marmo, o irmão que não tive biologicamente. Sua preocupação, o amor e o carinho com que cuida de mim são impossíveis de se agradecer e retribuir. Não há como não admirar a competência, o bom humor, a garra e a suavidade com que conduz sua vida. È uma grande fonte de inspiração para qualquer um que o conheça.

- Aos meus tios Nicolau e Sônia Marmo que sempre foram um porto seguro. É uma honra poder compartilhar com eles, quaisquer minutos que passemos juntos.

- Aos meus sogros, Antônio e Antônia Perroni, que são como se fossem meus pais.

- Aos meus amigos, Harley Sato e Cláudio Brunoro, pelo apoio e compreensão principalmente nos últimos meses. 
- Ao meu orientador, José R. C. Piqueira. É claro que é de praxe que um estudante agradeça ao seu professor... Mas o professor Piqueira sempre esteve comigo, nos melhores e piores momentos, sem me abandonar e proporcionando todo o suporte e apoio que foi preciso. Não há palavras que possam agradecê-lo adequadamente. Só posso desejar que esta tese tenha ficado à altura de sua competência como professor, pesquisador e orientador. Muito obrigado mestre!

Finalmente, gostaria de dedicar esta tese a duas pessoas:

- Ao meu pai, Carlos Marmo. Que saudades... Muitas saudades!

- Ao meu filho de 7 meses, Pedro Perroni Marmo. Que alegria... Espero que um dia você tenha orgulho desta tese que o papai fez para você!

Carlos Nehemy Marmo 


\section{RESUMO}

Este trabalho apresenta um estudo qualitativo das equações diferenciais nãolineares que descrevem o sincronismo de fase nos PLLs de $3^{\mathrm{a}}$ ordem que compõem redes OWMS de topologia mista, Estrela Simples e Cadeia Simples. O objetivo é determinar, através da Teoria de Bifurcações, os valores ou relações entre os parâmetros constitutivos da rede que permitam a existência e a estabilidade do estado síncrono, quando são aplicadas, no oscilador mestre, duas funções de excitação muito comuns na prática: o degrau e a rampa de fase.

$\mathrm{Na}$ determinação da estabilidade dos pontos de equilíbrio, sob o ponto de vista de Lyapunov, a existência de pontos de equilíbrio não-hiperbólicos não permite uma aproximação linear e, nesses casos, é aplicado o Teorema da Variedade Central. Essa técnica de simplificação de sistemas dinâmicos permite fazer uma aproximação homeomórfica em torno desses pontos, preservando a orientação no espaço de fases e possibilitando determinar localmente suas estabilidades. 


\begin{abstract}
This work presents a qualitative study of the non-linear differential equations that describe the synchronous state in 3rd order PLLs that compose One-way masterslave time distribution networks with Single Star and Single Chain topologies. Using bifurcation theory, the dynamical behavior of third-order phase-locked loops employed to extract the syncronous state in each node is analyzed depending on constitutive node parameters when two usual inputs, the step and the ramp phase pertubations, are supposed to appear in the master node.

When parameter combinations result in non hyperbolic synchronous states, from Lyapunov point of view, the linear approximation does not provide any information about the local behavior of the system. In this case, the center manifold theorem permits the construction of an equivalent vector field representing the asymptotic behavior of the original system in the neighborhood of these points. Thus, the local stability can be determined.
\end{abstract}




\section{SUMÁRIO}

\section{LISTA DE FIGURAS}

I. Introdução. . . . . . . . . . . . . . . . . . . . . . . . . 1

II. Estudo de um PLL de $3^{\mathrm{a}}$ ordem isolado submetido a variações no sinal de entrada do tipo degrau de fase e rampa de fase . . . . . . . . . . . . 7

2.1 Obtenção da equação diferencial que descreve a dinâmica de um PLL de $3^{\text {a }}$ ordem isolado . . . . . . . . . . . . . . . . . . .

2.2 Estabilidade frente à variação do tipo degrau de fase no sinal de entrada . . . . . . . . . . . . . . . . . . . . 13

2.2.1 Análise Linear da estabilidade dos pontos de equilíbrio

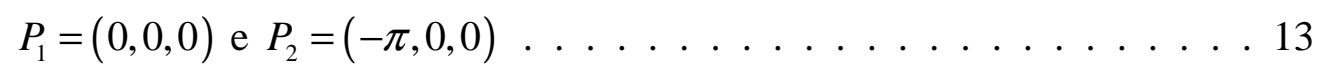

2.2.2 Análise da estabilidade de $P_{1}=(0,0,0)$ via TVC . . . . . . . . 24

2.2.3 Análise da estabilidade de $P_{2}=(-\pi, 0,0)$ via TVC . . . . . . . 19

2.3 Estabilidade frente à variação do tipo rampa de fase no sinal de entrada . . . . . . . . . . . . . . . . 2

2.3.1 Análise Linear da estabilidade de $P_{1}=\left(+\frac{\pi}{2}, 0,0\right) \ldots \ldots . \ldots .23$

2.3.2 Análise da estabilidade de $P_{1}=\left(+\frac{\pi}{2}, 0,0\right)$ via TVC . . . . . 32 
2.3.3 Análise Linear da estabilidade

de $P_{2,3}=\left(\arccos \left( \pm \sqrt{1-\left(\frac{\Omega}{\mu}\right)^{2}}\right), 0,0\right) \ldots \ldots \ldots \ldots$

2.3.4 Análise da estabilidade de $P=(0,0,0)$ via TVC . . . . . . . 32

III. Sistema dinâmico de um PLL de $3^{\mathrm{a}}$ ordem de uma rede

OWMS Estrela - Cadeia Simples . . . . . . . . . . . . . . . . 38

3.1 Classificação dos escravos quanto ao nível de

proximidade em relação ao mestre . . . . . . . . . . . . . . 38

3.2 Equação dinâmica de um escravo do nível I . . . . . . . . . . . . . . 39

3.3 Equação dinâmica de um escravo do nível II . . . . . . . . . . . . . . 41

3.4 Equação dinâmica de um escravo do nível III . . . . . . . . . . . . 44

3.5 Equação dinâmica de um escravo do nível VI . . . . . . . . . . . . .45

3.6 Equação dinâmica de um escravo do nível V . . . . . . . . . . . . . . 47

3.7 Equação dinâmica de um escravo qualquer . . . . . . . . . . . . . .47

3.8 Sistema dinâmico de nó escravo que não esteja

diretamente conectado ao mestre. . . . . . . . . . . . . . . . . . . .48

3.9 Sistema dinâmico para um mestre, um nó

escravo I e um nó escravo II . . . . . . . . . . . . . . . . . . . . . 51 
IV. Estudo da estabilidade de um nó escravo tipo II frente a uma variação do tipo degrau de fase no mestre . . . . . . . . . . . . . . . 54

4.1 Obtenção dos pontos de equilíbrio . . . . . . . . . . . . . . . . 54

\subsubsection{Análise Linear da estabilidade dos}

pontos de equilíbrio $P_{1}=(0,0,0,0,0,0)$,

$P_{2}=(-\pi, 0,0,0,0,0), P_{3}=(-\pi, 0,0,-\pi, 0,0) \mathrm{e}$

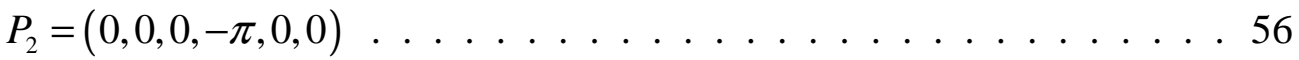

4.2.2 Análise da estabilidade de $P_{1}=(0,0,0,0,0,0)$ via TVC . . . . . 58

4.2.3 Análise da estabilidade de $P_{2}=(-\pi, 0,0,0,0,0)$ via TVC . . . . 65

4.2.4 Análise da estabilidade de $P_{3}=(-\pi, 0,0,-\pi, 0,0)$ via TVC . . . 72

4.2.5 Análise da estabilidade de $P_{4}=(0,0,0,-\pi, 0,0)$ via TVC . . . 80

V. Estudo da estabilidade de um nó escravo tipo II frente a uma variação do tipo rampa de fase no mestre . . . . . . . . . . . . . . 88

5.1 Obtenção dos pontos de equilíbrio . . . . . . . . . . . . . . . 88

5.2 Estudo do ponto $P=\left(+\frac{\pi}{2}, 0,0,-\pi, 0,0\right) \ldots \ldots \ldots \ldots \ldots \ldots \ldots \ldots \ldots$

5.2.1 Análise Linear da estabilidade. . . . . . . . . . . . . . . . . . . 91 
5.3 Estudo dos pontos

$$
P_{1,2}=\left(+\frac{\pi}{2}, 0,0,+\frac{\pi}{2}+\arccos \left[ \pm \sqrt{1-\left(\frac{\Omega}{\mu_{I I}}\right)^{2}}\right], 0,0\right) \ldots \ldots \ldots
$$

5.3.1 Análise Linear da estabilidade . . . . . . . . . . . . . . . . . 103

5.3.2 Análise via TVC . . . . . . . . . . . . . . . . 106

5.4 Estudo dos pontos

$$
P_{3,4}=\left(\arccos \left[ \pm \sqrt{1-\left(\frac{\Omega}{\mu_{I}}\right)^{2}}\right], 0,0,+\frac{\pi}{2}+\arccos \left[ \pm \sqrt{1-\left(\frac{\Omega}{\mu_{I}}\right)^{2}}\right], 0,0\right) \ldots .112
$$

5.4.1 Análise Linear da estabilidade . . . . . . . . . . . . . . . . 112

5.4 .2 Análise via TVC . . . . . . . . . . . . . . . . . 114

5.5 Estudo dos pontos

$$
P_{5,6}=\left(+\arccos \left[ \pm \sqrt{1-\left(\frac{\Omega}{\mu_{I}}\right)^{2}}\right], 0,0,+\arccos \left[ \pm \sqrt{1-\left(\frac{\Omega}{\mu_{I}}\right)^{2}}\right]+\arccos \left[ \pm \sqrt{1-\left(\frac{\Omega}{\mu_{I I}}\right)^{2}}\right], 0,0\right)
$$

5.5.1 Análise Linear da estabilidade . . . . . . . . . . . . . . . . . 128

5.5 .2 Análise via TVC . . . . . . . . . . . . . . . 130 
5.6 Estudo dos pontos

$$
\begin{array}{r}
P_{7,8}=\left(+\arccos \left[ \pm \sqrt{1-\left(\frac{\Omega}{\mu_{I}}\right)^{2}}\right], 0,0,+\arccos \left[ \pm \sqrt{1-\left(\frac{\Omega}{\mu_{I}}\right)^{2}}\right]+\arccos \left[\mp \sqrt{1-\left(\frac{\Omega}{\mu_{I I}}\right)^{2}}\right], 0,0\right) \\
\ldots \ldots \ldots \ldots \ldots \ldots \ldots \ldots \ldots \ldots \ldots \ldots \ldots \ldots \ldots \ldots \ldots \ldots \ldots \ldots \ldots \ldots
\end{array}
$$

5.6.1 Análise Linear da estabilidade . . . . . . . . . . . . . . . . 137

5.6 .2 Análise via TVC . . . . . . . . . . . . . . . . . . . 139

VI. Síntese dos resultados . . . . . . . . . . . . . . . . . . . . . . 146

6.1 Sincronismo para uma variação do tipo degrau de fase no sinal do mestre . . . . . . . . . . . . . . . . 146

6.1.2 Sincronismo de um nó tipo I . . . . . . . . . . . . . . . . . 146

6.1.3 Sincronismo de um nó tipo II . . . . . . . . . . . . . . . . . . 148

6.2 Sincronismo para uma variação do tipo rampa de fase no sinal do mestre . . . . . . . . . . . . . . . . . 153

6.2.1 Sincronismo de um nó tipo I . . . . . . . . . . . . . . . . 153

6.2.2 Sincronismo de um nó tipo II . . . . . . . . . . . . . . . 157

6.2.2.1 Estabilidade do ponto $\mathrm{P} \ldots \ldots$. . . . . . . . 158

6.2.2.2 Estabilidade do ponto $\mathrm{P}_{1,2} \ldots \ldots \ldots$. . . . . . 160 
6.2.2.3 Estabilidade do ponto $\mathrm{P}_{3,4} \ldots \ldots$. . . . . . . . . 161

6.2.2.4 Estabilidade do ponto $\mathrm{P}_{5,6} \ldots \ldots \ldots 2$

6.2.2.5 Estabilidade do ponto $\mathrm{P}_{7,8} \ldots \ldots$. . . . . . . . 162

VII. Conclusões . . . . . . . . . . . . . . . . . . . . . . . . . . . . . . . . . 164

REFERÊNCIAS BIBLIOGRÁFICAS. . . . . . . . . . . . . . . . . . 165 


\section{LISTA DE FIGURAS}

Figura 1 - Representação esquemática de uma rede OWMS genérica $\ldots$. . . . . .2

Figura 2.1 - Diagrama em blocos de um PLL . . . . . . . . . . . . .7

Figura 3.1 - Esquema simplificado dos níveis de proximidade dos escravos em relação ao mestre em redes OWMS Estrela - Cadeia Simples . . . . 38

Figura 3.2 - Diagrama em blocos de um escravo do nível $\mathrm{n}, \mathrm{n} \neq \mathrm{I}$, de uma rede OWMS. . . . . . . . . . . . . . . . . . . . . . . . . . . . 49

Figura 6.1 - Diagrama de bifurcações para P1. As setas indicam a evolução temporal de $\mathrm{x}(\mathrm{t})$, para um dado valor de e para uma dada condição inicial sobre a seta . . . . . . . . . . . . . . . . . . . . . . 146

Figura 6.2 - Diagrama de bifurcações para P2. As setas indicam a evolução temporal de $\mathrm{x}(\mathrm{t})$, para um dado valor de e para uma dada condição inicial sobre a seta . . . . . . . . . . . . . . . . . 146

Figura 6.3 - Retrato de fases em P1 e P2, para $\mu>0 \ldots \ldots \ldots \ldots$. . . . . . . . .

Figura 6.4 - Diagramas de bifurcações para $P_{1}$. As setas indicam a evolução temporal de $y(t) ; z(t)$, para um dado valor de $\mu_{I}$ e $\mu_{I I}$ e para uma dada condição inicial sobre a seta . . . . . . . . . . 149

Figura 6.6 - Diagramas de bifurcações para $\mathrm{P}_{2}$. As setas indicam a evolução temporal de $y(t) ; z(t)$, para um dado valor de $\mu_{I}$ e $\mu_{I I}$ e para uma dada condição inicial sobre a seta $\ldots \ldots \ldots \ldots$ 
Figura 6.7 - Diagramas de bifurcações para $P_{3}$. As setas indicam a evolução temporal de $\mathrm{y}(\mathrm{t}) ; \mathrm{z}(\mathrm{t})$, para um dado valor de $\mu_{I}$ e $\mu_{I I}$ e para uma dada condição inicial sobre a seta $\ldots . . . \ldots 151$

Figura 6.8 - Diagramas de bifurcações para $\mathrm{P}_{4}$. As setas indicam a evolução temporal de $\mathrm{y}(\mathrm{t}) ; \mathrm{z}(\mathrm{t})$, para um dado valor de $\mu_{I}$ e $\mu_{I I}$ e para uma dada condição inicial sobre a seta . . . . . . . . . . 152

Figura 6.9 - Diagrama de bifurcações para $\mathrm{P}_{1}$. As setas indicam a evolução temporal de $\mathrm{x}(\mathrm{t})$ para um dado valor de $(\mu,(a-c))$ e para uma dada condição inicial sobre a seta . . . . . . . . . . 154 Figura 6.10: Diagrama de bifurcações para o ponto $\mathrm{P}_{2,3}$. . . . . . . . . . 155 


\section{CAPÍTULO I}

\section{INTRODUÇÃO}

O estudo de Sistemas Dinâmicos tem sido ao longo dos tempos como um dos capítulos mais fascinantes do desenvolvimento da ciência e da tecnologia. O cientista francês Henri Poincaré (1854-1912) se destacou nessa área, na medida em que desenvolveu técnicas geométricas para o estudo qualitativo de equações diferenciais não-lineares. Nesse tipo de abordagem é possível obter conclusões qualitativas sobre a estabilidade dos pontos de equilíbrio de um sistema, através da análise de aproximações assintóticas. No entanto, informações quantitativas sobre o comportamento transiente do sistema são inevitavelmente perdidas nesse tipo de estudo (Monteiro, 2002).

Dentro do tema "sistemas dinâmicos", o estudo das redes de distribuição de sinais de tempo ocupa um lugar especial, devido a sua relevância cada vez maior na vida do ser humano. Uma rede de distribuição de sinais de tempo é composta por um determinado número de osciladores que são conectados segundo certa estratégia e que tem por finalidade executar a coordenação temporal entre os mesmos, denominada "sincronismo". Entende-se por sincronismo entre dois ou mais osciladores como sendo o estado em que a diferença entre suas frequiências torna-se nula, ou seja, suas diferenças de fases se mantêm constante. Seus osciladores, ou também chamados "nós", tanto podem ser fisicamente constituídos, como implementados em software (Orsatti, 2007). De acordo com a aplicação tecnológica ou o estudo científico em questão, diferentes arranjos de osciladores são possíveis. Entretanto, cada rede possui suas características próprias, sendo mais ou menos recomendada a esta ou àquela aplicação. Há diversas aplicações bastante usuais, podendo-se citar (Lindsey, 1985):

- Equipamentos eletrônicos que exijam controle automático de frequiência, tais como os televisores;

- Sistemas de Posicionamento Global, para fins de navegação e rastreamento;

- Processos de demodulação de sinais analógicos e digitais; 
- Estabelecimento de um sistema mundial de distribuição de sinais de tempo, propiciando as mais diversas aplicações, tais como aquelas relacionadas à coordenação de transportes ferroviários e marítimos;

- Sincronização de diversos relógios localizados em diferentes pontos de multiplexação em uma rede digital de comunicações;

- Controle e monitoração de desempenho em uma rede de controle de processos;

- Estabelecimento do sincronismo de um supercomputador constituído de múltiplos processadores.

Além dessas aplicações, outros estudos têm contribuído de forma bastante significativa para a compreensão de fenômenos específicos de outras áreas, como por exemplo, em biologia, podendo-se citar a modelagem de problemas relacionados ao sincronismo de osciladores biológicos (Bear, Connors e Paradiso, 2002; Lindsey, 1985; Marques e Menna-Barreto, 1997).

Dentre as redes utilizadas em engenharia, as redes Mestre-Escravo (MasterSlave - MS) se destacam no meio comercial, por apresentarem baixo custo e facilidade de implementação. Nas redes MS, considera-se que o nó mestre M é um oscilador extremamente preciso e independente, como por exemplo, um relógio atômico de Césio ou de Rubídio. Mais especificamente, as redes Mestre-Escravo estudadas neste trabalho são as redes OWMS, muito populares no meio técnico e comercial devido a sua simplicidade topológica. A designação OWMS ("One-Way Master-Slave”, do inglês, Rede Mestre-Escravo de Via Única) se deve ao fato de que a transmissão de sinais de tempo nessas redes ocorre num único sentido, isto é, sempre a partir do mestre.

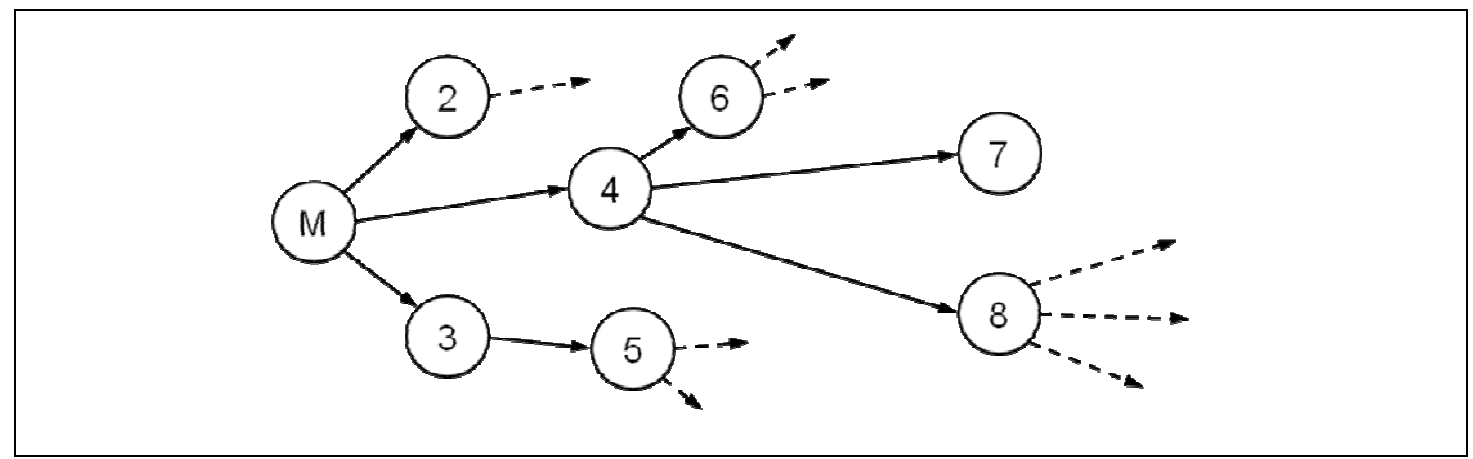

Fig. 1: Representação esquemática de uma rede OWMS genérica. 
No entanto, como é possível notar, o sincronismo nessas redes dependente essencialmente da integridade do mestre. Por este motivo, não são recomendadas em algumas aplicações, como por exemplo, para fins militares. Além disso, há estudos que demonstram que há uma tendência de propagação de perturbações pela hierarquia da rede, o que é certamente, uma desvantagem.

Além do mestre os dispositivos mais comumente utilizados para compor os demais nós da rede são os PLLs ou Malhas de Sincronismo de Fase ("Phase-locked Loop”). Como são controlados pelo mestre, estes nós são denominados escravos (Best, 1999; Gardner, 1980). Quando se estuda um PLL, há três perguntas iniciais que são muitas vezes complexas de serem respondidas:

- Um PLL não está sincronizado. Sob que condições ele sincroniza? Quanto tempo ele leva para sincronizar? Estas perguntas não serão respondidas por este trabalho de pesquisa;

- Um PLL está sincronizado. Em que condições ele permanece sincronizado? Para que isso seja possível, serão estabelecidas, neste trabalho, relações entre os parâmetros dos PLLs da rede e das funções de excitação propostas, que são o degrau e rampa de fase.

Os PLLs escolhidos para compor os osciladores escravos possuem duas características: em primeiro lugar são analógicos e em segundo, são de $3^{\text {a }}$ ordem. Apesar da crescente demanda por PLLs digitais, os PLLs analógicos ainda são muito utilizados, como por exemplo, em sintetizadores de frequiência (Margaris, 2004). Ainda, a escolha por PLLs de $3^{\mathrm{a}}$ ordem se deve às suas características próprias que são bastante interessantes em aplicações práticas, principalmente quando comparados aos PLLs de $1^{\mathrm{a}}$ ou $2^{\mathrm{a}}$ ordem. São características peculiares de um PLL de $3^{\mathrm{a}}$ ordem:

- Possuir maior atenuação dos termos de freqüência dupla na saída do detector de fases;

- Proporcionar melhor resposta a transientes, ou seja, uma captura mais rápida da freqüência de referência;

- Serem menos estáveis;

- Conseguir maior supressão de sinais espúrios. 
- Rastrear eficientemente mudanças de fase, acompanhando satisfatoriamente variações de fase em rampa, que é um dos objetos desta pesquisa.

Desconsiderou-se nesta pesquisa a influência dos termos de freqüência dupla, como é comum na literatura (Gardner, 1979; Piqueira, 1987). Piqueira e Monteiro (2003) demonstram a validade desta conduta para ganhos relativamente pequenos, sendo, portanto, possível de ser aqui adotada. Esta prerrogativa torna-se ainda mais aplicável a este estudo, devido a utilização de um filtro de $2^{\mathrm{a}}$ ordem que resulta em um PLL de $3^{\mathrm{a}}$ ordem.

Como este trabalho de pesquisa está voltado a um estudo qualitativo, isto é, verifica-se a estabilidade dos pontos de equilíbrio de um sistema dinâmico através da análise de aproximações assintóticas, informações sobre o comportamento transiente do sistema não podem, de modo geral, serem analisadas. Com a utilização de um PLL de $3^{\text {a }}$ ordem, a expectativa foi se esquivar desta preocupação.

Por outro lado, observa-se também que como todo PLL é um dispositivo prédisposto a apresentar não-linearidades, seu estudo sempre proporciona um rico material de pesquisa em Sistemas Dinâmicos. Em PLLs de $3^{\text {a }}$ ordem essa característica é ainda mais evidente.

O roteiro seguido para o estudo das condições de existência e estabilidade do sincronismo dos PLL deste trabalho foi:

$\left(1^{\circ}\right)$ Obter a equação ou o sistema de equações que descreve a dinâmica do PLL, composto basicamente de um detector de fases (considerado, por simplicidade, um multiplicador de sinais), um filtro de passa-baixa e um oscilador controlado por tensão. A escolha por um filtro de $2^{\mathrm{a}}$ ordem, genérico, com um zero e dois pólos, tem como objetivo alcançar uma boa solução de compromisso entre o comportamento em regime transitório e em regime estacionário (Ogata, 97). Atrasos de propagação de sinal não são aqui considerados por questão de simplicidade. As equações diferenciais obtidas são autônomas, não-lineares, não-homogêneas e de $3^{\text {a }}$ ordem;

$\left(2^{\circ}\right)$ Aplicar no oscilador mestre a função de excitação proposta, seja do tipo degrau, seja do tipo rampa de fase, obtendo a equação ou sistema de equações nessa situação; 
$\left(3^{\circ}\right)$ Reescrever a equação ou o sistema de equações diferenciais na forma de um sistema de equações no espaço de estados, a fim de se determinar os pontos equilíbrio, no sentido de Lyapunov. Quando, na análise linear, a parte real de um autovalor é negativa (positiva), o autovetor correspondente determina uma direção estável (instável). Caso todos os autovalores tenham parte real negativa, o ponto de equilíbrio é dito assintoticamente estável, no sentido de Lyapunov. Então, basta que pelo menos um autovalor tenha parte real positiva, para que o ponto de equilíbrio seja instável. Em ambos os casos, o ponto de equilíbrio é denominado hiperbólico. Para pontos hiperbólicos, o Teorema de Hartman-Grobman garante a equivalência topológica orbital entre o sistema original e o linearizado. Entretanto, se pelo menos um autovalor tiver parte real nula (ou mesmo for nulo), o ponto de equilíbrio é denominado não-hiperbólico, situação em que a análise linear falha na determinação da sua estabilidade. Assim, para pontos não-hiperbólicos, exige-se a aplicação de algum outro método para a determinação da estabilidade. Dentre aqueles existentes optou-se, neste trabalho, pelo Teorema da Variedade Central, que garante a existência de um homeomorfismo local que preserva a orientação no espaço de fases. O cálculo da assim chamada variedade central, tangente ao subespaço central, é possibilitado pela aplicação dos teoremas de Carr (Carr, 1981; Guckenheimer e Holmes, 1983; Fiedler-Ferrara e Prado, 1995). Através deles pode-se avaliar, via Teoria de Bifurcações, as mudanças na estabilidade estrutural do sistema em torno dos pontos de equilíbrio considerados, levando-se em conta as possíveis combinações dos valores dos parâmetros, tanto aqueles relativos ao PLL, quanto dos sinais de entrada e de saída (Wiggins, 1990; Guckenheimer e Holmes, 1983; Marmo, 2003);

A principal ferramenta utilizada para a manipulação de equações e objetos matemáticos foi o software Mathematica 6, que se mostrou muito eficiente neste tipo de análise. Este trabalho seria impossível sem este tipo de software.

No capítulo II, será estudada a estabilidade de um PLL de $3^{\text {a }}$ ordem, isolado, frente a variações na fase do sinal de entrada, tanto do tipo degrau, quanto rampa de fase. Os resultados que serão obtidos nessas duas análises serão relevantes para o estudo de nós específicos de uma rede OWMS. 
No capítulo III, procede-se a um estudo semelhante à primeira parte do capítulo II. Esse capítulo tem como finalidade obter as equações que descrevem o sincronismo de nós específicos de uma rede OWMS genérica, que serão classificados segundo a posição que ocupam em relação ao nó mestre.

No capitulo IV, serão realizados os estudos propriamente ditos sobre a estabilidade de um nó específico da rede, no caso de uma variação do tipo degrau de fase no mestre.

No capítulo V, o estudo do capítulo IV é realizado novamente, considerandose uma variação do tipo rampa de fase no mestre.

No capítulo VI, realiza-se uma síntese dos resultados deste trabalho de pesquisa tese, objetivando tomar conclusões comparativas entre as diferentes situações apresentadas.

No capítulo VII, procede-se a uma conclusão geral a respeito dos resultados apresentados no capítulo VI, evidenciando propostas para a continuidade deste trabalho. 
CAPÍTULO II

ESTUDO DE UM PLL DE 3 ${ }^{\text {a }}$ ORDEM ISOLADO SUBMETIDO A VARIAÇÕES NO SINAL DE ENTRADA DO TIPO DEGRAU DE FASE E RAMPA DE FASE

Neste capítulo estuda-se a estabilidade de um PLL de $3^{\text {a }}$ ordem, isolado, frente a variações na fase do sinal de entrada. Primeiramente, será obtida a equação diferencial que descreve a dinâmica do PLL. De posse dessa equação, será realizado o estudo da sua estabilidade frente a uma variação na fase do tipo degrau no seu sinal de entrada. Em seguida, repete-se o estudo para uma variação do tipo rampa de fase. Os resultados que serão obtidos nessas duas análises serão relevantes para o estudo de nós específicos de uma rede OWMS, o que ocorrerá nos próximos capítulos.

\subsection{OBTENÇÃO DA EQUAÇÃO DIFERENCIAL QUE DESCREVE A DINÂMICA DE UM PLL DE $3^{\text {a }}$ ORDEM ISOLADO}

O diagrama em blocos do PLL proposto neste trabalho pode ser esquematizado como segue (Piqueira, 1987):

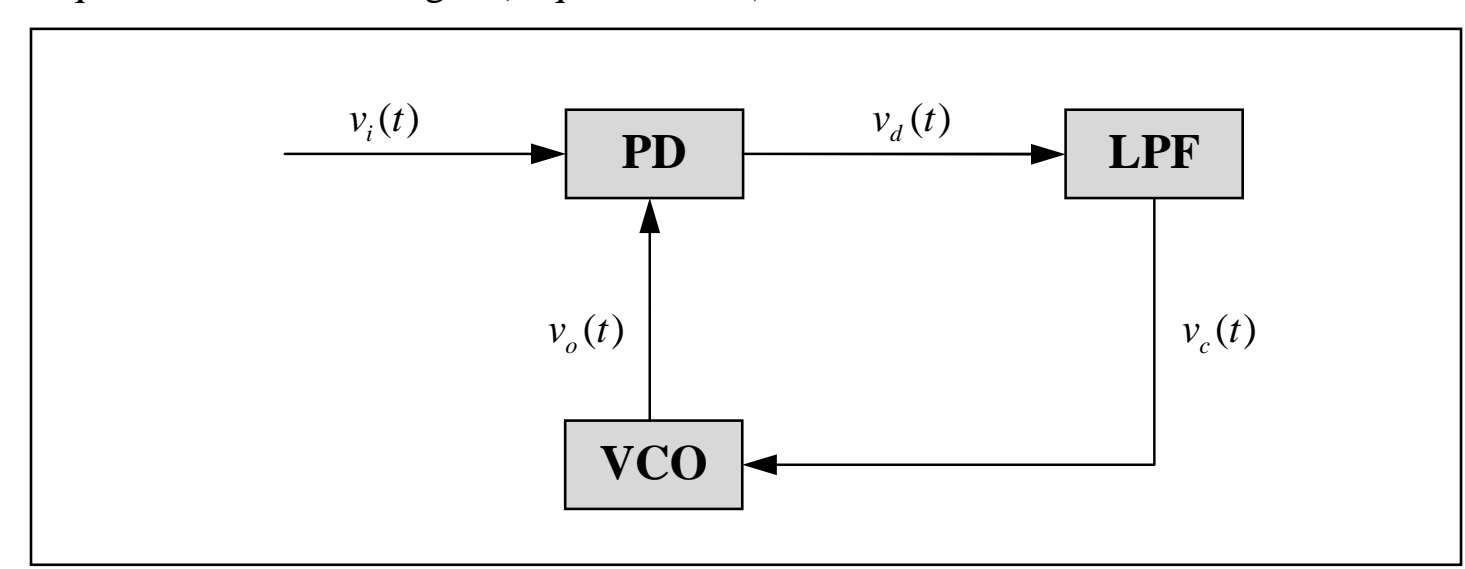

Fig. 2.1: Diagrama em blocos de um PLL.

Há três blocos indicados neste diagrama:

- Detector de Fases ou PD (do inglês, "Phase Detector”);

- Filtro Passa-Baixas ou LPF (do inglês, “Low-Pass Filter”);

- Oscilador Controlado por Tensão ou VCO (do inglês, "Voltage Controlled Oscillator”). 
O sinal de entrada $v_{i}(t)$ (do inglês, "in") é também denominado sinal externo ou de referência. Este sinal é considerado periódico e pode ser descrito pela seguinte equação:

$$
v_{i}(t)=V_{i} \cdot \operatorname{sen} \Phi_{i}(t)=V_{i} \cdot \operatorname{sen}\left[\omega_{0} \cdot t+\theta_{i}(t)\right]
$$

Em que:

- $\quad V_{i}$ é a amplitude do sinal, suposta constante e positiva;

- $\Phi_{i}(t)$ é a fase do sinal, variável no tempo;

- $\omega_{0}$ é a freqüência angular central do sinal que, por simplificação, é suposta constante no tempo, positiva e de mesmo valor para qualquer sinal de entrada que se considere;

- $\theta_{i}(t)$ é a mudança de fase do sinal, variável no tempo.

O sinal de saída $v_{o}(t)$ (em inglês, out) é também denominado sinal local. Este sinal é considerado periódico e pode ser descrito pela seguinte equação:

$$
v_{o}(t)=V_{o} \cdot \cos \Phi_{o}(t)=V_{o} \cdot \cos \left[\omega_{0} \cdot t+\theta_{o}(t)\right]
$$

Em que:

- $\quad V_{o}$ é a amplitude do sinal, suposta constante e positiva;

- $\Phi_{o}(t)$ é a fase do sinal, variável no tempo;

- $\omega_{0}$ é a frequiência angular central do sinal que, por simplificação, é suposta constante, positiva e idêntica à do sinal de entrada $v_{i}(t)$;

- $\theta_{o}(t)$ é a mudança de fase do sinal, variável no tempo.

Deve-se observar que os sinais são periódicos, mas são descritos por funções diferentes, seno e cosseno, ou seja, em quadratura, para que seja possível escrever o sinal $v_{d}(t)$ como a diferença das suas fases.

A cada instante, o PD compara a fase do sinal local com a fase do sinal externo. Esse elemento será, por simplicidade, considerado um multiplicador de sinais. Sendo assim, pode-se escrever a sua saída $v_{d}(t)$, que pode ser denominada na prática de Erro de Fase Dinâmico, como sendo:

$$
v_{d}(t)=K_{m} \cdot v_{i}(t) \cdot v_{o}(t)
$$


Em (2.3), $K_{m}$ é o ganho do PD ou também chamado de Fator de Multiplicação do PD.

Substituindo (2.1) e (2.2) em (2.3), tem-se:

$$
v_{d}(t)=K_{m} \cdot V_{i} \cdot \operatorname{sen}\left[\omega_{0} \cdot t+\theta_{i}(t)\right] \cdot V_{o} \cdot \cos \left[\omega_{0} \cdot t+\theta_{o}(t)\right]
$$

Por simplicidade algébrica, cada termo trigonométrico de (2.4) é desenvolvido em separado, como segue:

$$
\begin{aligned}
& \operatorname{sen}\left[\omega_{0} \cdot t+\theta_{i}(t)\right]=\operatorname{sen}\left(\omega_{0} \cdot t\right) \cdot \cos \theta_{i}(t)+\cos \left(\omega_{0} \cdot t\right) \cdot \operatorname{sen} \theta_{i}(t) \\
& \cos \left[\omega_{0} \cdot t+\theta_{o}(t)\right]=\cos \left(\omega_{0} \cdot t\right) \cdot \cos \theta_{o}(t)-\operatorname{sen}\left(\omega_{0} \cdot t\right) \cdot \operatorname{sen} \theta_{o}(t)
\end{aligned}
$$

Substituindo os termos (2.5) e (2.6) em (2.4), tem-se:

$$
\begin{aligned}
v_{d}(t)= & K_{m} \cdot V_{i} \cdot\left[\operatorname{sen}\left(\omega_{0} \cdot t\right) \cdot \cos \theta_{i}(t)+\cos \left(\omega_{0} \cdot t\right) \cdot \operatorname{sen} \theta_{i}(t)\right] \\
& \cdot V_{o} \cdot\left[\cos \left(\omega_{0} \cdot t\right) \cdot \cos \theta_{o}(t)-\operatorname{sen}\left(\omega_{0} \cdot t\right) \cdot \operatorname{sen} \theta_{o}(t)\right]
\end{aligned}
$$

Desenvolvendo a multiplicação dos fatores de (2.7) que estão entre colchetes, tem-se:

$$
\begin{aligned}
v_{d}(t)=K_{m} \cdot V_{i} \cdot V_{o} \cdot & {\left[\operatorname{sen}\left(\omega_{0} \cdot t\right) \cdot \cos \theta_{i}(t) \cdot \cos \left(\omega_{0} \cdot t\right) \cdot \cos \theta_{o}(t)+\right.} \\
+ & \cos \left(\omega_{0} \cdot t\right) \cdot \operatorname{sen} \theta_{i}(t) \cdot \cos \left(\omega_{0} \cdot t\right) \cdot \cos \theta_{o}(t)- \\
& -\operatorname{sen}\left(\omega_{0} \cdot t\right) \cdot \cos \theta_{i}(t) \cdot \operatorname{sen}\left(\omega_{0} \cdot t\right) \cdot \operatorname{sen} \theta_{o}(t)- \\
& \left.-\cos \left(\omega_{0} \cdot t\right) \cdot \operatorname{sen} \theta_{i}(t) \cdot \operatorname{sen}\left(\omega_{0} \cdot t\right) \cdot \operatorname{sen} \theta_{o}(t)\right]
\end{aligned}
$$

Simplificando (2.8), tem-se:

$$
\begin{aligned}
v_{d}(t)=K_{m} \cdot V_{i} \cdot V_{o} \cdot & {\left[\operatorname{sen}\left(\omega_{0} \cdot t\right) \cdot \cos \left(\omega_{0} \cdot t\right) \cdot \cos \theta_{i}(t) \cdot \cos \theta_{o}(t)+\right.} \\
+ & \cos ^{2}\left(\omega_{0} \cdot t\right) \cdot \operatorname{sen} \theta_{i}(t) \cdot \cos \theta_{o}(t)- \\
- & \operatorname{sen}^{2}\left(\omega_{0} \cdot t\right) \cdot \cos \theta_{i}(t) \cdot \operatorname{sen} \theta_{o}(t)- \\
& \left.-\operatorname{sen}\left(\omega_{0} \cdot t\right) \cdot \cos \left(\omega_{0} \cdot t\right) \cdot \operatorname{sen} \theta_{i}(t) \cdot \operatorname{sen} \theta_{o}(t)\right]
\end{aligned}
$$

Então, fazendo as devidas transformações trigonométricas em (2.9), tem-se: 


$$
\begin{aligned}
v_{d}(t)=K_{m} \cdot V_{i} \cdot V_{o} & \cdot\left\{\frac{\operatorname{sen}\left(2 \omega_{0} \cdot t\right)}{2} \cdot \cos \theta_{i}(t) \cdot \cos \theta_{o}(t)+\right. \\
& +\left[\frac{1}{2}+\frac{1}{2} \cos \left(2 \omega_{0} \cdot t\right)\right] \cdot \operatorname{sen} \theta_{i}(t) \cdot \cos \theta_{o}(t)- \\
& -\left[\frac{1}{2}-\frac{1}{2} \cos \left(2 \omega_{0} \cdot t\right)\right] \cdot \cos \theta_{i}(t) \cdot \operatorname{sen} \theta_{o}(t)- \\
& \left.-\frac{\operatorname{sen}\left(2 \omega_{0} \cdot t\right)}{2} \cdot \operatorname{sen} \theta_{i}(t) \cdot \operatorname{sen} \theta_{o}(t)\right\}
\end{aligned}
$$

Desenvolvendo a multiplicação dos fatores de (2.10) que estão entre colchetes, tem-se:

$$
\begin{aligned}
v_{d}(t)=\frac{K_{m} \cdot V_{i} \cdot V_{o}}{2} \cdot\left[\operatorname{sen}\left(2 \omega_{0} \cdot t\right) \cdot \cos \theta_{i}(t) \cdot \cos \theta_{o}(t)+\right. \\
\quad+\operatorname{sen} \theta_{i}(t) \cdot \cos \theta_{o}(t)+\cos \left(2 \omega_{0} \cdot t\right) \cdot \operatorname{sen} \theta_{i}(t) \cdot \cos \theta_{o}(t)- \\
\quad-\cos \theta_{i}(t) \cdot \operatorname{sen} \theta_{o}(t)+\cos \left(2 \omega_{0} \cdot t\right) \cdot \cos \theta_{i}(t) \cdot \operatorname{sen} \theta_{o}(t)- \\
\left.\quad-\operatorname{sen}\left(2 \omega_{0} \cdot t\right) \cdot \operatorname{sen} \theta_{i}(t) \cdot \operatorname{sen} \theta_{o}(t)\right]
\end{aligned}
$$

Simplificando (2.11):

$$
\begin{aligned}
v_{d}(t)=\frac{K_{m} \cdot V_{i} \cdot V_{o}}{2} & \\
& \cdot\left\{\operatorname{sen}\left(2 \omega_{0} \cdot t\right) \cdot\left[\cos \theta_{i}(t) \cdot \cos \theta_{o}(t)-\operatorname{sen} \theta_{i}(t) \cdot \operatorname{sen} \theta_{o}(t)\right]+\right. \\
& +\cos \left(2 \omega_{0} \cdot t\right) \cdot\left[\operatorname{sen} \theta_{i}(t) \cdot \cos \theta_{o}(t)+\cos \theta_{i}(t) \cdot \operatorname{sen} \theta_{o}(t)\right] \\
& \left.+\operatorname{sen} \theta_{i}(t) \cdot \cos \theta_{o}(t)-\cos \theta_{i}(t) \cdot \operatorname{sen} \theta_{o}(t)\right\}
\end{aligned}
$$

Neste estudo, assume-se, como é comum na literatura, que os termos de freqüência dupla de (2.12) podem ser eliminados pelo LPF (Gardner, 1979). Estudos mais recentes (Piqueira, Monteiro, 2003) demonstram a validade desta conduta para ganhos relativamente pequenos, o que é plenamente aplicável a este estudo. Então, desprezando-se o termo de freqüência dupla, a expressão (2.12) pode ser simplificada, obtendo-se:

$$
v_{d}(t)=\frac{K_{m} \cdot V_{i} \cdot V_{o}}{2} \cdot \operatorname{sen}\left(\theta_{i}(t)-\theta_{o}(t)\right)
$$


O sinal descrito em (2.13) será, então, aplicado ao filtro passa-baixas (LPF) a fim de se eliminar os componentes de alta freqüência.

Segundo a proposta deste trabalho, utiliza-se um LPF de $2^{\mathrm{a}}$ ordem, com dois pólos e um zero. Então, a função de transferência do filtro pode ser descrita por:

$$
F(s)=\frac{V_{c}(s)}{V_{d}(s)}=\frac{a \cdot s+1}{b \cdot s^{2}+c \cdot s+1}
$$

A utilização de um filtro de $2^{\mathrm{a}}$ ordem faz com que o PLL seja de $3^{\mathrm{a}}$ ordem, uma ordem a mais que o filtro.

Através da aplicação da inversa da transformada de Laplace em (2.14), com condições iniciais nulas, pode-se obter:

$$
b \cdot \ddot{v}_{c}(t)+c \cdot \dot{v}_{c}(t)+v_{c}(t)=a \cdot \dot{v}_{d}(t)+v_{d}(t)
$$

Por outro lado, a equação que descreve a dinâmica do VCO é dada por:

$$
\dot{\theta}_{o}(t)=K \cdot v_{c}(t), \text { ou seja, } v_{c}(t)=\frac{\dot{\theta}_{o}(t)}{K}
$$

Em (2.16), $K$ é o ganho do VCO.

Derivando (2.16) em relação ao tempo, obtém-se:

$$
\dot{v}_{c}(t)=\frac{\ddot{\theta}_{o}(t)}{K} \Rightarrow \ddot{v}_{c}(t)=\frac{\dddot{\theta}_{o}(t)}{K}
$$

Utilizando (2.17) em (2.15), tem-se:

$$
\frac{b}{K} \cdot \dddot{\theta}_{o}(t)+\frac{c}{K} \cdot \ddot{\theta}_{o}(t)+\frac{1}{K} \cdot \dot{\theta}_{o}(t)=a \cdot \dot{v}_{d}(t)+v_{d}(t)
$$

Antes de proceder ao desenvolvimento de (2.17) é necessário derivar (2.13) em relação ao tempo, obtendo:

$$
\dot{v}_{d}(t)=a \cdot \frac{K_{m} \cdot V_{i} \cdot V_{o}}{2} \cdot\left(\dot{\theta}_{i}(t)-\dot{\theta}_{o}(t)\right) \cdot \cos \left(\theta_{i}(t)-\theta_{o}(t)\right)
$$

Utilizando-se as expressões de (2.13) e (2.19) em (2.18), tem-se:

$$
\begin{aligned}
\frac{b}{K} \cdot \dddot{\theta}_{o}(t) & +\frac{c}{K} \cdot \ddot{\theta}_{o}(t)+\frac{1}{K} \cdot \dot{\theta}_{o}(t)= \\
= & a \cdot \frac{K_{m} \cdot V_{i} \cdot V_{o}}{2} \cdot\left(\dot{\theta}_{i}(t)-\dot{\theta}_{o}(t)\right) \cdot \cos \left(\theta_{i}(t)-\theta_{o}(t)\right)+ \\
& +\frac{K_{m} \cdot V_{i} \cdot V_{o}}{2} \cdot \operatorname{sen}\left(\theta_{i}(t)-\theta_{o}(t)\right)
\end{aligned}
$$


A seguir, para fins de simplificação, define-se o seguinte parâmetro:

$$
\mu=\frac{\Delta \cdot K_{m} \cdot V_{i} \cdot V_{o}}{2}
$$

Dessa forma, reescreve-se (2.20) como sendo:

$$
\begin{gathered}
b \cdot \dddot{\theta}_{o}(t)+c \cdot \ddot{\theta}_{o}(t)+\dot{\theta}_{o}(t)=a \cdot \mu \cdot\left(\dot{\theta}_{i}(t)-\dot{\theta}_{o}(t)\right) \cdot \cos \left(\theta_{i}(t)-\theta_{o}(t)\right)+ \\
+\mu \cdot \operatorname{sen}\left(\theta_{i}(t)-\theta_{o}(t)\right)
\end{gathered}
$$

Para descrever a estabilidade do nó, define-se o seguinte o erro de fase:

$$
\varphi(t) \stackrel{\Delta}{=} \Phi_{i}(t)-\Phi_{o}(t), \quad \varphi(t) \in[-\pi, \pi[
$$

Em (2.23), observa-se que $\Phi_{i}(t) \stackrel{\Delta}{=} \omega_{0} \cdot t+\theta_{i}(t)$ e $\Phi_{o}(t) \stackrel{\Delta}{=} \omega_{0} \cdot t+\theta_{o}(t)$. Ainda, que $\varphi(t)$ é a diferença de fase entre os sinais de entrada e de saída. Dessa forma, pode-se reescrever (2.23) como sendo:

$$
\varphi(t)=\theta_{i}(t)-\theta_{o}(t)
$$

Derivando (2.24) em relação ao tempo, obtém-se:

$$
\dot{\varphi}(t)=\dot{\theta}_{i}(t)-\dot{\theta}_{o}(t) \Rightarrow \ddot{\varphi}(t)=\ddot{\theta}_{i}(t)-\ddot{\theta}_{o}(t) \Rightarrow \dddot{\varphi}(t)=\dddot{\theta}_{i}(t)-\dddot{\theta}_{o}(t)
$$

Utilizando (2.24) e (2.25) em (2.22), obtém-se:

$$
\begin{aligned}
& b \cdot\left(\dddot{\theta}_{i}(t)-\dddot{\varphi}(t)\right)+c \cdot\left(\ddot{\theta}_{i}(t)-\ddot{\varphi}(t)\right)+\left(\dot{\theta}_{i}(t)-\dot{\varphi}(t)\right)= \\
& =a \cdot \mu \cdot \dot{\varphi}(t) \cdot \cos \varphi(t)+\mu \cdot \operatorname{sen} \varphi(t)
\end{aligned}
$$

Assim, pode-se finalmente escrever a equação que descreve a dinâmica do PLL considerado, como:

$$
\begin{gathered}
b \cdot \dddot{\varphi}(t)+c \cdot \ddot{\varphi}(t)+\dot{\varphi}(t)+a \cdot \mu \cdot \dot{\varphi}(t) \cdot \cos \varphi(t)+\mu \cdot \operatorname{sen} \varphi(t)= \\
=b \cdot \dddot{\theta}_{i}(t)+c \cdot \ddot{\theta}_{i}(t)+\dot{\theta}_{i}(t)
\end{gathered}
$$

A seguir, serão estudadas as estabilidades dos pontos de equilíbrio desse sistema dinâmico frente a variações no sinal de entrada. 


\subsection{ESTABILIDADE FRENTE À VARIAÇÃO DO TIPO DEGRAU DE FASE NO SINAL DE ENTRADA.}

Para variações $\theta_{i}(t)$ do tipo degrau de fase, pode-se escrever que:

$$
\dot{\theta}_{i}(t)=0 \Rightarrow \ddot{\theta}_{i}(t)=0 \Rightarrow \dddot{\theta}_{i}(t)=0
$$

Substituindo as entradas descritas em (2.28) na equação (2.27) que descreve a dinâmica do PLL, tem-se:

$$
b \cdot \dddot{\varphi}(t)+c \cdot \ddot{\varphi}(t)+\dot{\varphi}(t)+a \cdot \mu \cdot \dot{\varphi}(t) \cdot \cos \varphi(t)+\mu \cdot \operatorname{sen} \varphi(t)=0
$$

Para a obtenção do sistema de equações no espaço de estados são definidas três variáveis de estado: $x_{1}=\varphi, x_{2}=\dot{\varphi}$ e $x_{3}=\ddot{\varphi}$. Dessa forma, para $b \neq 0$, tem-se:

$$
\left\{\begin{array} { l } 
{ x _ { 1 } = \varphi } \\
{ x _ { 2 } = \dot { \varphi } } \\
{ x _ { 3 } = \ddot { \varphi } }
\end{array} \Rightarrow \left\{\begin{array}{l}
\dot{x}_{1}=x_{2} \\
\dot{x}_{2}=x_{3} \\
\dot{x}_{3}=-\frac{c}{b} x_{3}-\frac{1}{b} x_{2}-\frac{a}{b} \mu \cdot x_{2} \cdot \cos x_{1}-\frac{\mu}{b} \operatorname{sen} x_{1}
\end{array}\right.\right.
$$

Os pontos de equilíbrio de (2.30) podem ser obtidos como segue:

$$
\left\{\begin{array}{l}
\dot{x}_{1}=0 \Rightarrow x_{2}^{*}=0 \\
\dot{x}_{2}=0 \Rightarrow x_{3}^{*}=0 \\
\dot{x}_{3}=0 \quad \Rightarrow \quad-\frac{\mu}{b} \cdot \operatorname{sen} x_{1}^{*}=0 \Rightarrow\left\{\begin{array}{l}
x_{1}^{*}=0 \\
\text { ou } \\
x_{1}^{*}=-\pi
\end{array}\right.
\end{array}\right.
$$

Portanto, os pontos de equilíbrio de (2.30) são:

$$
P_{1}=(0,0,0) \text { e } P_{2}=(-\pi, 0,0)
$$

A estabilidade destes pontos será discutida nas próximas subseções.

\subsubsection{ANÁLISE LINEAR DA ESTABILIDADE DOS PONTOS DE} EQUILÍBRIO $P_{1}=(0,0,0)$ E $P_{2}=(-\pi, 0,0)$

A matriz Jacobiana de (2.30), calculada nos pontos de equilíbrio, é: 


$$
J_{P_{1,2}}=\left[\begin{array}{ccc}
0 & 1 & 0 \\
0 & 0 & 1 \\
\mp \frac{\mu}{b} & -\frac{1}{b} \mp \frac{\mu \cdot a}{b} & -\frac{c}{b}
\end{array}\right]
$$

Os autovalores de (2.32) são:

- $\lambda_{1}=-\frac{c}{3 b}+\frac{\psi}{3 \sqrt[3]{2} b}-\frac{\sqrt[3]{2}\left(-c^{2}+3 b+3 \mu \cdot a \cdot b\right)}{\psi}$

- $\quad \lambda_{2}=-\frac{c}{3 b} \pm \frac{(1-\sqrt{3} i) \psi}{6 \sqrt[3]{2} b} \mp \frac{(1+\sqrt{3} i)\left(-c^{2}+3 b+3 \mu \cdot a \cdot b\right)}{3 \sqrt[3]{4} \psi}$

- $\lambda_{3}=-\frac{c}{3 b} \pm \frac{(1+\sqrt{3} i) \psi}{6 \sqrt[3]{2} b} \mp \frac{(1-\sqrt{3} i)\left(-c^{2}+3 b+3 \mu \cdot a \cdot b\right)}{3 \sqrt[3]{4} \psi}$

Nas expressões dos autovalores de (2.33), considera-se que:

- $\psi=\sqrt[3]{\eta+\xi}$

- $\quad \eta=-2 c^{3}+9 b \cdot c \pm 9 \mu \cdot a \cdot b \cdot c \mp 27 \mu \cdot b^{2}$

- $\xi=\sqrt{4\left(-c^{2}+3 \cdot b \pm 3 \mu \cdot a \cdot b\right)^{3}+\eta^{2}}$.

O próximo passo na análise linear da estabilidade trata da obtenção da parte real dos autovalores. Verifica-se para quais combinações de parâmetros $(a, b, c, \mu)$ cada autovalor pode assumir parte real negativa, nula ou positiva. Observando as expressões dos autovalores em (2.33), nota-se que há uma dificuldade considerável em proceder a essa análise analiticamente. Um outro modo seria fazer uma análise numérica. Como este trabalho tem como objetivo fazer estudos analíticos que proporcionem conclusões gerais a respeito da estabilidade dos PLLs, esta opção não será levada em conta. A alternativa encontrada foi simplificar o sistema dinâmico, transformando o parâmetro $\mu$ em variável de estado através da inclusão de uma nova equação diferencial sem dinâmica, ou seja, $\dot{\mu}=0$. Assim, o sistema (2.30) passa a se apresentar como: 


$$
\left\{\begin{array}{l}
\dot{x}_{1}=x_{2} \\
\dot{x}_{2}=x_{3} \\
\dot{x}_{3}=-\frac{c}{b} x_{3}-\frac{1}{b} x_{2}-\frac{a}{b} \mu \cdot x_{2} \cdot \cos x_{1}-\frac{\mu}{b} \operatorname{sen} x_{1}
\end{array}\right.
$$

Então, a matriz Jacobiana de (2.34), calculada em $P_{1,2}$, é:

$$
J_{P_{1,2}}=\left[\begin{array}{rrr}
0 & 1 & 0 \\
0 & 0 & 1 \\
0 & -\frac{1}{b} & -\frac{c}{b}
\end{array}\right]
$$

Por simplicidade, a linha e a coluna de (2.35) correspondentes à nova variável $\mu$ estão sendo omitidas, uma vez que a sua introdução não alterou a dinâmica do sistema.

Assim, os autovalores de (2.35) são:

- $\quad \lambda_{1}=0$

- $\lambda_{2}=-\frac{\left(c+\sqrt{c^{2}-4 b}\right)}{2 b}$

- $\lambda_{3}=\frac{\left(\sqrt{c^{2}-4 b}-c\right)}{2 b}$

Através da análise dos autovalores de (2.35), pode-se verificar que:

- O autovalor $\lambda_{1}$ é nulo independentemente dos valores ou relações que possam assumir os parâmetros envolvidos.

- Os autovalores $\lambda_{2}$ e $\lambda_{3}$ são ou reais com parte real negativa, para $c^{2} \geq 4 b$, ou pares de complexos conjugados com parte real negativa, para $c^{2}<4 b$.

Portanto, graças à existência de um autovalor nulo, não é possível obter conclusões sobre a estabilidade de $\mathrm{P}$, através da análise linear. Para investigar o 
comportamento do sistema no subespaço central, será aplicado o Teorema da Variedade Central (TVC).

\subsubsection{ANÁLISE DA ESTABILIDADE DE $P_{1}=(0,0,0)$ VIA TVC}

Para a aplicação do TVC, faz-se necessária a expansão em série de Taylor das funções seno e co-seno em torno de $x_{1}^{*}=0$ :

$$
\operatorname{sen} x_{1} \approx x_{1}-\frac{x_{1}^{3}}{6}+\ldots \text { e } \cos x_{1} \approx 1-\frac{x_{1}^{2}}{2}+\ldots
$$

Com as expansões de (2.37), reescreve-se o sistema (2.34), já na forma matricial, ou seja, $\dot{X}=J_{P_{1,2}} \cdot X+F$ e $\dot{\mu}=0$, como segue:

$$
\begin{aligned}
& {\left[\begin{array}{l}
\dot{x}_{1} \\
\dot{x}_{2} \\
\dot{x}_{3}
\end{array}\right]=\left[\begin{array}{ccc}
0 & 1 & 0 \\
0 & 0 & 1 \\
0 & -\frac{1}{b} & -\frac{c}{b}
\end{array}\right]\left[\begin{array}{c}
x_{1} \\
x_{2} \\
x_{3}
\end{array}\right]+\left(\begin{array}{c}
0 \\
0 \\
-\frac{a \cdot \mu}{b} x_{2}\left(1-\frac{x_{1}^{2}}{2}\right)-\frac{\mu}{b}\left(x_{1}-\frac{x_{1}^{3}}{6}\right)
\end{array}\right)} \\
& \text { е } \dot{\mu}=0
\end{aligned}
$$

Conforme requerido pelo TVC, o sistema (2.38) precisa ser reescrito na forma canônica de Jordan. Para isso, define-se um novo conjunto de variáveis de estado $V=\left(v_{1}, v_{2}, v_{3}\right)^{T}$ de modo que $X=M \cdot V$, sendo $M$ uma base composta pelos autovetores de $J_{P_{1,2}}$ de (2.35). Dessa forma, é possível dizer que $V=\left[M^{-1} \cdot J_{P_{1,2}} \cdot M\right] \cdot V+M^{-1} \cdot F$, ou seja:

$$
\begin{aligned}
& {\left[\begin{array}{l}
\dot{v}_{1} \\
\dot{v}_{1} \\
\dot{v}_{3}
\end{array}\right]=\left[\begin{array}{ccc}
0 & 0 & 0 \\
0 & -\frac{\left(c_{i}+\sqrt{c_{i}^{2}-4 b_{i}}\right)}{2 b_{i}} & 0 \\
0 & 0 & \frac{\left(\sqrt{c_{i}^{2}-4 b_{i}}-c_{i}\right)}{2 b_{i}}
\end{array}\right] \cdot\left[\begin{array}{l}
v_{1} \\
v_{2} \\
v_{3}
\end{array}\right]+\left[\begin{array}{l}
f_{1} \\
f_{2} \\
f_{3}
\end{array}\right]} \\
& \text { e } \dot{\mu}=0
\end{aligned}
$$


Em (2.39), tem-se que:

- $f_{1}=\frac{1}{6} \mu\left\{\left[v_{1}+\frac{1}{2}(c \cdot \delta-2 b) v_{2}+(c \cdot \varepsilon-2 b) v_{3}\right]^{3}-6 v_{1}+\right.$
$+3(2 b-c \cdot \delta) v_{2}+3(2 b-c \cdot \varepsilon) v_{3}-$
$\left.-3 a\left(-\delta \cdot v_{2}-\varepsilon \cdot v_{3}\right)\left[1-\frac{1}{8}\left(2 v_{1}+(c \cdot \delta-2 b) v_{2}+(c \cdot \varepsilon-2 b) v_{3}\right)^{2}\right]\right\}$

- $f_{2}=\frac{\varepsilon \cdot f_{1}}{2 b \sqrt{c^{2}-4 b}}$

- $f_{3}=\frac{-\delta \cdot f_{1}}{2 b \sqrt{c^{2}-4 b}}$

- $\delta=c-\sqrt{c^{2}-4 b}$

- $\varepsilon=c+\sqrt{c^{2}-4 b}$

Então, de acordo com o Teorema da Variedade Central (Guckenheimer e Holmes, 1983; Wiggins, 1990; Monteiro, 2002), procura-se a variedade central $W^{c}$, que seja tangente ao subespaço central $E^{c}$ no ponto de equilíbrio $P_{1}$. Assim, para $x$ e $\mu$ suficientemente pequenos, tem-se:

$$
\begin{aligned}
W^{c}(0)=\{ & \left(v_{1}, v_{2}, v_{3}, \mu\right) \in \mathfrak{R}^{4} / v_{1}=x, v_{2}=h_{1}(x, \mu), v_{3}=h_{2}(x, \mu), \\
& \left.h_{j}(0,0)=0, D h_{j}(0,0)=0, j=1,2\right\}
\end{aligned}
$$

Considere-se então, os seguintes polinômios estimadores $h_{1}(x, \mu)$ e $h_{2}(x, \mu)$, compostos por monômios do segundo grau:

$$
\begin{cases}h_{1}(x, \mu)=\alpha_{1} \cdot x^{2}+\alpha_{2} \cdot x \cdot \mu+\alpha_{3} \cdot \mu^{2}, & \left(\alpha_{1}, \alpha_{2}, \alpha_{3}\right) \in \mathfrak{R} \\ h_{2}(x, \mu)=\beta_{1} \cdot x^{2}+\beta_{2} \cdot x \cdot \mu+\beta_{3} \cdot \mu^{2}, & \left(\beta_{1}, \beta_{2}, \beta_{3}\right) \in \mathfrak{R}\end{cases}
$$

Pelo teorema de Carr (Carr, 1981; Guckenheimer e Holmes, 1983; Wiggins, 1990; Monteiro, 2002), a variedade central $W^{c}$ deve satisfazer $N(h(x, \mu))=0$. Portanto, tem-se: 


$$
\left\{\begin{array}{r}
\frac{\partial h_{1}(x, \mu)}{\partial x}\left[\lambda_{1} \cdot x+f_{1}\left(x, h_{1}(x, \mu), h_{2}(x, \mu), \mu\right)\right]- \\
-\lambda_{2} \cdot h_{2}(x, \mu)-f_{2}\left(x, h_{1}(x, \mu), h_{2}(x, \mu), \mu\right)=0 \\
\begin{array}{rl}
\frac{\partial h_{2}(x, \mu)}{\partial x}\left[\lambda_{1} \cdot x+f_{1}\left(x, h_{1}(x, \mu), h_{2}(x, \mu), \mu\right)\right]- \\
-\lambda_{3} \cdot h_{2}(x, \mu)-f_{3}\left(x, h_{1}(x, \mu), h_{2}(x, \mu), \mu\right)=0
\end{array}
\end{array}\right.
$$

Utilizando (2.39) e (2.41) em (2.42), tem-se:

$$
\left\{\begin{array}{l}
\left(2 \alpha_{1} \cdot x+\alpha_{2} \cdot \mu\right) \cdot\left[0+f_{1}\right]-\lambda_{2} \cdot\left(\alpha_{1} \cdot x^{2}+\alpha_{2} \cdot x \cdot \mu+\alpha_{3} \cdot \mu^{2}\right)-f_{2}=0 \\
\left(2 \beta_{1} \cdot x+\beta_{2} \cdot \mu\right) \cdot\left[0+f_{1}\right]-\lambda_{3} \cdot\left(\beta_{1} \cdot x^{2}+\beta_{2} \cdot x \cdot \mu+\beta_{3} \cdot \mu^{2}\right)-f_{3}=0
\end{array}\right.
$$

Pela resolução de (2.43) é possível obter, igualando-se os termos de igual potência, os coeficientes de $h_{1}(x, \mu)$ e $h_{2}(x, \mu)$ :

$$
\begin{aligned}
& \left\{\begin{array}{l}
x^{2}: \quad \alpha_{1}=0 \\
\mu \cdot x: \quad \alpha_{2}=\frac{\sqrt{c^{2}-4 b}}{4 b-c^{2}} \\
\mu^{2}: \quad \alpha_{3}=0
\end{array}\right. \\
& \left\{\begin{array}{l}
x^{2}: \quad \beta_{1}=0 \\
\mu \cdot x: \quad \beta_{2}=\frac{\sqrt{c^{2}-4 b}}{c^{2}-4 b} \\
\mu^{2}: \quad \beta_{3}=0
\end{array} \Rightarrow h_{1}(x, \mu)=\frac{\sqrt{c^{2}-4 b}}{4 b-c^{2}} \cdot x \cdot \mu\right.
\end{aligned}
$$

Substituindo (2.44) e (2.45) no sistema (2.39), pode-se obter, desprezando os termos de ordem superior, o campo de vetores restrito à variedade central:

$$
\left\{\begin{array}{l}
\dot{x}=-\mu \cdot x \\
\dot{\mu}=0
\end{array}\right.
$$


Para obter o ponto de equilíbrio de (2.46), basta fazer:

$$
\begin{aligned}
& \dot{x}=-\mu \cdot x \\
& \dot{x}=0
\end{aligned} \Rightarrow x^{*}=0
$$

Verifica-se sua estabilidade derivando parcialmente (2.46) em relação à $x$ :

$$
\frac{\partial(\dot{x})}{\partial x}=-\mu
$$

De (2.48) conclui-se que:

- Para $\mu>0$, o ponto de equilíbrio é assintoticamente estável;

- Para $\mu<0$, o ponto de equilíbrio é instável.

\subsubsection{ANÁLISE DA ESTABILIDADE DE $P_{2}=(-\pi, 0,0)$ VIA TVC}

Da mesma forma que em (2.2.2), para a aplicação do TVC, faz-se necessária a expansão em série de Taylor das funções das funções seno e co-seno, em torno de $x_{1}^{*}=-\pi$ :

$$
\operatorname{sen} x_{1} \approx-\left(x_{1}+\pi\right)+\frac{\left(x_{1}+\pi\right)^{3}}{6}+\ldots \text { e } \cos x_{1} \approx-1+\frac{\left(x_{1}+\pi\right)^{2}}{2}+\ldots
$$

Entretanto, o TVC exige que o ponto de equilíbrio esteja na origem. Portanto, para transladar o sistema de coordenadas, considere-se a seguinte mudança de variáveis:

$$
\bar{x}_{1}=x_{1}+\pi, \bar{x}_{2}=x_{2} \text { e } \bar{x}_{3}=x_{3}
$$

Então, o sistema (2.34), já transladado para a origem e na forma matricial, pode ser reescrito como segue:

$$
\begin{aligned}
& {\left[\begin{array}{c}
\dot{\bar{x}}_{1} \\
\dot{\bar{x}}_{2} \\
\dot{\bar{x}}_{3}
\end{array}\right]=\left[\begin{array}{ccc}
0 & 1 & 0 \\
0 & 0 & 1 \\
0 & -\frac{1}{b} & -\frac{c}{b}
\end{array}\right]\left[\begin{array}{c}
0 \\
\bar{x}_{1} \\
\bar{x}_{2}
\end{array}\right]+\left(\begin{array}{c}
0 \\
-\frac{a \cdot \mu}{b} \bar{x}_{2}\left(-1+\frac{\bar{x}_{1}^{2}}{2}\right)-\frac{\mu}{b}\left(-\bar{x}_{1}+\frac{\bar{x}_{1}^{3}}{6}\right)
\end{array}\right)} \\
& \text { e } \dot{\mu}=0
\end{aligned}
$$

A expressão (2.52) pode ser representada por $\dot{\bar{X}}=J_{P_{1,2}} \cdot \bar{X}+\bar{F}$ e $\dot{\mu}=0$. 
Conforme requerido pelo TVC, o sistema (2.52) precisa ser reescrito na forma canônica de Jordan. Para isso, define-se um novo conjunto de variáveis de estado $V=\left(v_{1}, v_{2}, v_{3}\right)^{T}$ de modo que $\bar{X}=M \cdot V$, sendo $M$ uma base composta pelos autovetores de $J_{P_{1,2}}$ de (2.35). Dessa forma, é possível dizer que $V=\left[M^{-1} \cdot J_{P_{1,2}} \cdot M\right] \cdot V+M^{-1} \cdot \bar{F}$, ou seja:

$$
\left[\begin{array}{c}
\dot{v}_{1} \\
\dot{v}_{1} \\
\dot{v}_{3}
\end{array}\right]=\left[\begin{array}{ccc}
0 & 0 & 0 \\
0 & -\frac{\left(c_{i}+\sqrt{c_{i}^{2}-4 b_{i}}\right)}{2 b_{i}} & 0 \\
0 & 0 & \frac{\left(\sqrt{c_{i}^{2}-4 b_{i}}-c_{i}\right)}{2 b_{i}}
\end{array}\right] \cdot\left[\begin{array}{c}
v_{1} \\
v_{2} \\
v_{3}
\end{array}\right]+\left[\begin{array}{l}
\bar{f}_{1} \\
\overline{f_{2}} \\
\overline{f_{3}}
\end{array}\right]
$$

Em (2.53), tem-se que:

$$
\begin{aligned}
& \overline{f_{1}}=\mu\left\{-\frac{1}{48}\left[2 v_{1}+(c \cdot \delta-2 b) v_{2}+(c \cdot \varepsilon-2 b) v_{3}\right]^{3}+v_{1}+\right. \\
& +\frac{1}{2}(c \cdot \delta-2 b) v_{2}+\frac{1}{2}(c \cdot \varepsilon-2 b) v_{3}- \\
& \left.-\frac{1}{2} a\left(-\delta \cdot v_{2}-\varepsilon \cdot v_{3}\right)\left[\frac{1}{8}\left(2 v_{1}+(c \cdot \delta-2 b) v_{2}+(c \cdot \varepsilon-2 b) v_{3}\right)^{2}-1\right]\right\} \\
& \overline{f_{2}}=\frac{\varepsilon \cdot \overline{f_{1}}}{2 b \sqrt{c^{2}-4 b}} \\
& \overline{f_{3}}=\frac{-\delta \cdot \overline{f_{1}}}{2 b \sqrt{c^{2}-4 b}} \\
& \delta=c-\sqrt{c^{2}-4 b} \\
& \mathcal{E}=c+\sqrt{c^{2}-4 b}
\end{aligned}
$$

Da mesma forma que em (2.2.2), procura-se a variedade central $W^{c}$ que seja tangente ao subespaço central $E^{c}$ no ponto de equilíbrio $P_{2}$. Assim, para $x$ e $\mu$ suficientemente pequenos, tem-se: 


$$
\begin{aligned}
W^{c}(0)=\{ & \left(v_{1}, v_{2}, v_{3}, \mu\right) \in \mathfrak{R}^{4} / v_{1}=x, v_{2}=h_{1}(x, \mu), v_{3}=h_{2}(x, \mu), \\
& \left.h_{j}(0,0)=0, D h_{j}(0,0)=0, j=1,2\right\}
\end{aligned}
$$

Considere-se então, os seguintes polinômios estimadores $h_{1}(x, \mu)$ e $h_{2}(x, \mu)$, compostos por monômios do segundo grau:

$$
\begin{cases}h_{1}(x, \mu)=\alpha_{1} \cdot x^{2}+\alpha_{2} \cdot x \cdot \mu+\alpha_{3} \cdot \mu^{2}, & \left(\alpha_{1}, \alpha_{2}, \alpha_{3}\right) \in \mathfrak{R} \\ h_{2}(x, \mu)=\beta_{1} \cdot x^{2}+\beta_{2} \cdot x \cdot \mu+\beta_{3} \cdot \mu^{2}, & \left(\beta_{1}, \beta_{2}, \beta_{3}\right) \in \mathfrak{R}\end{cases}
$$

Pelo teorema de Carr (Carr, 1981; Guckenheimer e Holmes, 1983; Wiggins, 1990; Monteiro, 2002), a variedade central $W^{c}$ deve satisfazer $N(h(x, \mu))=0$. Portanto, tem-se:

$$
\left\{\begin{array}{r}
\frac{\partial h_{1}(x, \mu)}{\partial x}\left[\lambda_{1} \cdot x+\bar{f}_{1}\left(x, h_{1}(x, \mu), h_{2}(x, \mu), \mu\right)\right]- \\
\quad-\lambda_{2} \cdot h_{2}(x, \mu)-\bar{f}_{2}\left(x, h_{1}(x, \mu), h_{2}(x, \mu), \mu\right)=0 \\
\begin{array}{rl}
\frac{\partial h_{2}(x, \mu)}{\partial x}\left[\lambda_{1} \cdot x+\bar{f}_{1}\right. & \left.\left(x, h_{1}(x, \mu), h_{2}(x, \mu), \mu\right)\right]- \\
& -\lambda_{3} \cdot h_{2}(x, \mu)-\bar{f}_{3}\left(x, h_{1}(x, \mu), h_{2}(x, \mu), \mu\right)=0
\end{array}
\end{array}\right.
$$

Utilizando (2.53) e (2.55) em (2.56), tem-se:

$$
\begin{aligned}
& \left(2 \alpha_{1} \cdot x+\alpha_{2} \cdot \mu\right) \cdot\left[0+\bar{f}_{1}\right]-\lambda_{2} \cdot\left(\alpha_{1} \cdot x^{2}+\alpha_{2} \cdot x \cdot \mu+\alpha_{3} \cdot \mu^{2}\right)-\bar{f}_{2}=0 \\
& \left(2 \beta_{1} \cdot x+\beta_{2} \cdot \mu\right) \cdot\left[0+\tilde{f}_{1}\right]-\lambda_{3} \cdot\left(\beta_{1} \cdot x^{2}+\beta_{2} \cdot x \cdot \mu+\beta_{3} \cdot \mu^{2}\right)-\overline{f_{3}}=0
\end{aligned}
$$

Pela resolução de (2.57) é possível obter, igualando-se os termos de igual potência, os coeficientes de $h_{1}(x, \mu)$ e $h_{2}(x, \mu)$ :

$$
\left\{\begin{array}{l}
x^{2}: \quad \alpha_{1}=0 \\
\mu \cdot x: \quad \alpha_{2}=\frac{\sqrt{c^{2}-4 b}}{c^{2}-4 b} \\
\mu^{2}: \quad \alpha_{3}=0
\end{array} \Rightarrow h_{1}(x, \mu)=\frac{\sqrt{c^{2}-4 b}}{c^{2}-4 b} \cdot x \cdot \mu\right.
$$




$$
\left\{\begin{array}{l}
x^{2}: \quad \beta_{1}=0 \\
\mu \cdot x: \quad \beta_{2}=\frac{\sqrt{c^{2}-4 b}}{4 b-c^{2}} \quad \Rightarrow \quad h_{2}(x, \mu)=\frac{\sqrt{c^{2}-4 b}}{4 b-c^{2}} \cdot x \cdot \mu \\
\mu^{2}: \quad \beta_{3}=0
\end{array}\right.
$$

Substituindo (2.58) e (2.59) no sistema (2.53), pode-se obter, desprezando os termos de ordem superior, o campo de vetores restrito à variedade central:

$$
\left\{\begin{array}{l}
\dot{x}=+\mu \cdot x \\
\dot{\mu}=0
\end{array}\right.
$$

Para obter o ponto de equilíbrio de (2.60), basta fazer:

$$
\begin{aligned}
& \dot{x}=+\mu \cdot x \\
& \dot{x}=0
\end{aligned} \Rightarrow x^{*}=0
$$

Verifica-se sua estabilidade derivando parcialmente (2.60) em relação à $x$ :

$$
\frac{\partial(\dot{x})}{\partial x}=+\mu
$$

De (2.62) conclui-se que:

- Para $\mu>0$, o ponto de equilíbrio é instável;

- Para $\mu<0$, o ponto de equilíbrio é assintoticamente estável.

\subsection{ESTABILIDADE FRENTE À VARIAÇÃO DO TIPO RAMPA DE FASE NO SINAL DE ENTRADA.}

Para variações $\theta_{i}(t)$ do tipo rampa de fase, pode-se escrever:

$$
\theta_{i}(t)=\Omega \cdot t+\theta_{0} \Rightarrow \dot{\theta}_{i}(t)=\Omega \Rightarrow \ddot{\theta}_{i}(t)=0 \Rightarrow \dddot{\theta}_{i}(t)=0
$$

Substituindo as entradas descritas em (2.64) na equação (2.27) que descreve a dinâmica do PLL, tem-se:

$$
b \cdot \dddot{\varphi}(t)+c \cdot \ddot{\varphi}(t)+\dot{\varphi}(t)+a \cdot \mu \cdot \dot{\varphi}(t) \cdot \cos \varphi(t)+\mu \cdot \operatorname{sen} \varphi(t)=\Omega
$$

Para a obtenção do sistema de equações no espaço de estados são definidas três variáveis de estado $x_{1}=\varphi, x_{2}=\dot{\varphi}$ e $x_{3}=\ddot{\varphi}$. Dessa forma, para $b \neq 0$, tem-se: 


$$
\left\{\begin{array} { l } 
{ x _ { 1 } = \varphi } \\
{ x _ { 2 } = \dot { \varphi } } \\
{ x _ { 3 } = \ddot { \varphi } }
\end{array} \Rightarrow \left\{\begin{array}{l}
\dot{x}_{1}=x_{2} \\
\dot{x}_{2}=x_{3} \\
\dot{x}_{3}=\frac{\Omega}{b}-\frac{c}{b} x_{3}-\frac{1}{b} x_{2}-\frac{a}{b} \mu \cdot x_{2} \cdot \cos x_{1}-\frac{\mu}{b} \operatorname{sen} x_{1}
\end{array}\right.\right.
$$

Os pontos de equilíbrio de (2.66) podem ser obtidos como segue:

$$
\begin{aligned}
& \left\{\begin{array}{l}
\dot{x}_{1}=0 \Rightarrow x_{2}^{*}=0 \\
\dot{x}_{2}=0 \Rightarrow x_{3}^{*}=0 \\
\dot{x}_{3}=0 \Rightarrow \frac{\Omega}{b}-\frac{\mu}{b} \cdot \operatorname{sen} x_{1}^{*}=0 \Rightarrow \operatorname{sen} x_{1}^{*}=\frac{\Omega}{\mu}, \mu \neq 0
\end{array}\right. \\
& \therefore\left\{\begin{array}{l}
x_{1}^{*}=+\frac{\pi}{2}, \text { se } \Omega=\mu \\
\text { ou } \\
x_{1}^{*}=\arccos \left[ \pm \sqrt{1-\left(\frac{\Omega}{\mu}\right)^{2}}\right], \text { se } \Omega<\mu
\end{array}\right.
\end{aligned}
$$

Portanto, os pontos de equilíbrio são:

- Se $\Omega=\mu$, então $P_{1}=\left(+\frac{\pi}{2}, 0,0\right)$

- Se $\Omega<\mu$, então $P_{2,3}=\left(\arccos \left( \pm \sqrt{1-\left(\frac{\Omega}{\mu}\right)^{2}}\right), 0,0\right)$

A estabilidade destes pontos será discutida nas próximas subseções.

2.3.1 ANÁLISE LINEAR DA ESTABILIDADE DE $P_{1}=\left(+\frac{\pi}{2}, 0,0\right)$

A matriz Jacobiana de (2.66), calculada no ponto de equilíbrio $P_{1}=\left(+\frac{\pi}{2}, 0,0\right)$, é: 


$$
J_{P_{1}}=\left[\begin{array}{rrr}
0 & 1 & 0 \\
0 & 0 & 1 \\
0 & -\frac{1}{b} & -\frac{c}{b}
\end{array}\right]
$$

Assim, os autovalores de $J_{P_{1}}$ são:

- $\lambda_{1}=0$

- $\lambda_{2}=-\frac{\left(c+\sqrt{c^{2}-4 b}\right)}{2 b}$

- $\lambda_{3}=\frac{\left(\sqrt{c^{2}-4 b}-c\right)}{2 b}$

Através da análise idêntica a ocorrida em (2.36), pode-se verificar que:

- $\mathrm{O}$ autovalor $\lambda_{1}$ é nulo, independentemente dos valores ou relações que possam assumir os parâmetros envolvidos.

- Os autovalores $\lambda_{2}$ e $\lambda_{3}$ são ou reais com parte real negativa, para $c^{2} \geq 4 b$, ou pares de complexos conjugados com parte real negativa, para $c^{2}<4 b$.

Portanto, graças à existência de um autovalor nulo, não é possível obter conclusões sobre a estabilidade de $P_{1}$, através da análise linear. Para investigar o comportamento do sistema no subespaço central, será aplicado o Teorema da Variedade Central (TVC) na seção seguinte.

\subsubsection{ANÁLISE DA ESTABILIDADE DE $P_{1}=\left(+\frac{\pi}{2}, 0,0\right)$ VIA TVC}

Para a aplicação do TVC, faz-se necessária a expansão em série de Taylor das funções seno e co-seno em torno de $x_{1}^{*}=+\frac{\pi}{2}:$ sen $x_{1} \approx 1-\frac{1}{2}\left(x_{1}-\frac{\pi}{2}\right)^{2}+\ldots$ e $\cos x_{1} \approx-\left(x_{1}-\frac{\pi}{2}\right)+\ldots$ Dessa forma, reescreve-se o sistema (2.66) na forma matricial como segue: 


$$
\left[\begin{array}{l}
\dot{x}_{1} \\
\dot{x}_{2} \\
\dot{x}_{3}
\end{array}\right]=\left[\begin{array}{ccc}
0 & 1 & 0 \\
0 & 0 & 1 \\
0 & -\frac{1}{b} & -\frac{c}{b}
\end{array}\right]\left[\begin{array}{l}
x_{1} \\
x_{2} \\
x_{3}
\end{array}\right]+\left(\begin{array}{c}
0 \\
0 \\
\frac{a \cdot \mu}{b} x_{2}\left(x_{1}-\frac{\pi}{2}\right)+\frac{\mu}{2 b}\left(x_{1}-\frac{\pi}{2}\right)^{2}
\end{array}\right)
$$

A expressão (2.72) pode ser representada por $\dot{X}=J_{P_{1,2}} \cdot X+F$.

Como o TVC exige que o ponto de equilíbrio esteja na origem, é preciso transladar o sistema de coordenadas. Assim, considere-se a seguinte mudança de variáveis:

$$
\bar{x}_{1}=x_{1}-\frac{\pi}{2}, \bar{x}_{2}=x_{2} \text { e } \bar{x}_{3}=x_{3}
$$

Então, o sistema (2.71), já transladado para a origem e na forma matricial, pode ser reescrito como segue:

$$
\left[\begin{array}{c}
\dot{\bar{x}}_{1} \\
\dot{\bar{x}}_{2} \\
\dot{\bar{x}}_{3}
\end{array}\right]=\left[\begin{array}{ccc}
0 & 1 & 0 \\
0 & 0 & 1 \\
0 & -\frac{1}{b} & -\frac{c}{b}
\end{array}\right]\left[\begin{array}{c}
\bar{x}_{1} \\
\bar{x}_{2} \\
\bar{x}_{3}
\end{array}\right]+\left(\begin{array}{c}
0 \\
0 \\
\frac{a \cdot \mu}{b} \bar{x}_{2} \bar{x}_{1}+\frac{\mu}{2 b} \bar{x}_{1}^{2}
\end{array}\right)
$$

A expressão (2.73) pode ser representada por $\dot{\bar{X}}=J_{P_{1,2}} \cdot \bar{X}+\bar{F}$.

Conforme requerido pelo TVC, o sistema (2.73) precisa ser reescrito na forma canônica de Jordan. Para isso, define-se um novo conjunto de variáveis de estado $V=\left(v_{1}, v_{2}, v_{3}\right)^{T}$ de modo que $\bar{X}=M \cdot V$, sendo $M$ uma base composta pelos autovetores de $J_{P_{1,2}}$ de (2.69). Dessa forma, é possível dizer que $V=\left[M^{-1} \cdot J_{P_{1,2}} \cdot M\right] \cdot V+M^{-1} \cdot \bar{F}$, ou seja: 


$$
\left[\begin{array}{c}
\dot{v}_{1} \\
\dot{v}_{1} \\
\dot{v}_{3}
\end{array}\right]=\left[\begin{array}{ccc}
0 & 0 & 0 \\
0 & -\frac{\left(c+\sqrt{c^{2}-4 b}\right)}{2 b} & 0 \\
0 & 0 & \frac{\left(\sqrt{c^{2}-4 b}-c\right)}{2 b}
\end{array}\right] \cdot\left[\begin{array}{c}
v_{1} \\
v_{2} \\
v_{3}
\end{array}\right]+\left[\begin{array}{c}
\overline{f_{1}} \\
\overline{f_{2}} \\
\overline{f_{3}}
\end{array}\right]
$$

Sendo que em (2.74), tem-se:

$$
\begin{aligned}
& \bar{f}_{1}=\frac{1}{8} \mu\left[2 v_{1}+(c \cdot \delta-2 b) v_{2}+(c \cdot \varepsilon-2 b) v_{3}\right] \cdot\left\{2 v_{1}+\right. \\
& \left.+[-2 b-(c-2 a)(-c \cdot \delta-2 b)] v_{2}+[(c-2 a)(c \cdot \varepsilon-2 b)] v_{3}\right\} \\
& \bar{f}_{2}=\frac{\varepsilon \cdot \bar{f}_{1}}{2 b \sqrt{c^{2}-4 b}} \\
& \bar{f}_{3}=\frac{-\delta \cdot \overline{f_{1}}}{2 b \sqrt{c^{2}-4 b}} \\
& \delta=c-\sqrt{c^{2}-4 b} \\
& \varepsilon=c+\sqrt{c^{2}-4 b}
\end{aligned}
$$

Da mesma forma que em (2.2.2) e (2.2.3), procura-se a variedade central $W^{c}$ que seja tangente ao subespaço central $E^{c}$ no ponto de equilíbrio $P_{2}$. Assim, para $x$ e $\mu$ suficientemente pequenos, tem-se:

$$
\begin{aligned}
W^{c}(0)=\{ & \left(v_{1}, v_{2}, v_{3}\right) \in \mathfrak{R}^{3} / v_{1}=x, v_{2}=h_{1}(x), v_{3}=h_{2}(x), \\
& \left.h_{j}(0)=0, D h_{j}(0)=0, j=1,2\right\}
\end{aligned}
$$

Considere-se então, os seguintes polinômios estimadores $h_{1}(x)$ e $h_{2}(x)$ :

$$
\begin{cases}h_{1}(x)=\alpha_{1} \cdot x^{3}+\alpha_{2} \cdot x^{2}, & \left(\alpha_{1}, \alpha_{2}\right) \in \Re \\ h_{2}(x)=\beta_{1} \cdot x^{3}+\beta_{2} \cdot x^{2}, & \left(\beta_{1}, \beta_{2}\right) \in \Re\end{cases}
$$

Pelo teorema de Carr (Carr, 1981; Guckenheimer e Holmes, 1983; Wiggins, 1990; Monteiro, 2002), a variedade central $W^{c}$ deve satisfazer $N(h(x, \mu))=0$. Portanto, tem-se: 


$$
\left\{\begin{array}{l}
\frac{\partial h_{1}(x)}{\partial x}\left[\lambda_{1} \cdot x+f_{1}\left(x, h_{1}(x), h_{2}(x)\right)\right]-\lambda_{2} \cdot h_{2}(x)-f_{2}\left(x, h_{1}(x), h_{2}(x)\right)=0 \\
\frac{\partial h_{2}(x)}{\partial x}\left[\lambda_{1} \cdot x+f_{1}\left(x, h_{1}(x), h_{2}(x)\right)\right]-\lambda_{3} \cdot h_{2}(x)-f_{3}\left(x, h_{1}(x), h_{2}(x)\right)=0
\end{array}\right.
$$

Utilizando (2.74) e (2.76) em (2.77), tem-se:

$$
\begin{aligned}
& \left(3 \alpha_{1} \cdot x^{2}+2 \alpha_{2} \cdot x\right) \cdot\left[0+\bar{f}_{1}\right]-\lambda_{2} \cdot\left(\alpha_{1} \cdot x^{3}+\alpha_{2} \cdot x^{2}\right)-\bar{f}_{2}=0 \\
& \left(3 \beta_{1} \cdot x^{2}+2 \beta_{2} \cdot x\right) \cdot\left[0+\overline{f_{1}}\right]-\lambda_{3} \cdot\left(\beta_{1} \cdot x^{3}+\beta_{2} \cdot x^{2}\right)-\bar{f}_{3}=0
\end{aligned}
$$

Pela resolução de (2.78) é possível obter, igualando-se os termos de igual potência, os coeficientes de $h_{1}(x)$ e $h_{2}(x)$ :

$$
\begin{aligned}
& \left\{\begin{array}{l}
x^{3}: \quad \alpha_{1}=\frac{\mu^{2} \cdot\left(-c^{2}+4 b-2 a \sqrt{c^{2}-4 b}+3 c \sqrt{c^{2}-4 b}\right)}{4\left(4 b-c^{2}\right)} \\
x^{2}: \quad \alpha_{2}=-\frac{\mu \sqrt{c^{2}-4 b}}{2\left(4 b-c^{2}\right)}
\end{array}\right. \\
& \therefore h_{1}(x)=\frac{\mu^{2} \cdot\left(-c^{2}+4 b-2 a \sqrt{c^{2}-4 b}+3 c \sqrt{c^{2}-4 b}\right)}{4\left(4 b-c^{2}\right)} \cdot x^{3}-\frac{\mu \sqrt{c^{2}-4 b}}{2\left(4 b-c^{2}\right)} \cdot x^{2} \\
& \left\{\begin{array}{l}
x^{3}: \quad \beta_{1}=\frac{\mu^{2} \cdot\left(-c^{2}+4 b+2 a \sqrt{c^{2}-4 b}-3 c \sqrt{c^{2}-4 b}\right)}{4\left(4 b-c^{2}\right)} \\
x^{2}: \quad \beta_{2}=\frac{\mu \sqrt{c^{2}-4 b}}{2\left(4 b-c^{2}\right)}
\end{array}\right. \\
& \therefore h_{2}(x)=\frac{\mu^{2} \cdot\left(-c^{2}+4 b+2 a \sqrt{c^{2}-4 b}-3 c \sqrt{c^{2}-4 b}\right)}{4\left(4 b-c^{2}\right)} \cdot x^{3}+\frac{\mu \sqrt{c^{2}-4 b}}{2\left(4 b-c^{2}\right)} \cdot x^{2}
\end{aligned}
$$

Substituindo (2.79) e (2.80) no sistema (2.74), pode-se obter, desprezando os termos de ordem superior, o campo de vetores restrito à variedade central:

$$
\dot{x}=\frac{\mu^{2}(a-c)}{2} x^{3}+\frac{\mu}{2} x^{2}
$$

Para obter os pontos de equilíbrio, basta fazer $\dot{x}=0$ em (2.81): 


$$
\begin{aligned}
& \dot{x}=\frac{\mu^{2}(a-c)}{2} x^{3}+\frac{\mu}{2} x^{2} \\
& \dot{x}=0
\end{aligned} \Rightarrow\left\{\begin{array}{l}
x_{1}^{*}=-\frac{1}{(a-c) \mu}, a \neq c, \mu \neq 0 \\
x_{2}^{*}=x_{3}^{*}=0
\end{array}\right.
$$

Verifica-se suas estabilidades derivando parcialmente (2.81) em relação à $x$ :

$$
\frac{\partial \dot{x}}{\partial x}=+\mu \cdot x+\frac{3 \mu^{2}(a-c) x^{2}}{2}
$$

De (2.82) em (2.81), tem-se que:

$$
\left.\frac{\partial \dot{x}}{\partial x}\right|_{x_{1}^{*}}=+\frac{1}{2(a-c)} \text { e }\left.\frac{\partial \dot{x}}{\partial x}\right|_{x_{2,3}^{*}}=0
$$

De (2.84), conclui-se que:

- Para $a-c>0$, o ponto de equilíbrio $x_{1}^{*}$ é instável;

- Para $a-c<0$, o ponto de equilíbrio $x_{1}^{*}$ é assintoticamente estável.

$$
P_{2,3}=\left(\arccos \left( \pm \sqrt{1-\left(\frac{\Omega}{\mu}\right)^{2}}\right), 0,0\right)
$$

A matriz Jacobiana de (2.66), calculada nos pontos de equilíbrio $P_{2,3}=\left(\arccos \left( \pm \sqrt{1-\left(\frac{\Omega}{\mu}\right)^{2}}\right), 0,0\right)$,é:

$$
J_{P_{2,3}}=\left(\begin{array}{ccc}
0 & 1 & 0 \\
0 & 0 & 1 \\
-\frac{\mu}{b}\left[ \pm \sqrt{1-\left(\frac{\Omega}{\mu}\right)^{2}}\right] & -\frac{1}{b}-\frac{\mu \cdot a}{b}\left[ \pm \sqrt{1-\left(\frac{\Omega}{\mu}\right)^{2}}\right] & -\frac{c}{b}
\end{array}\right)
$$


Assim, os autovalores de $J_{P_{2,3}}$ são:

- $\lambda_{1}=-\frac{c}{3 b}+\frac{\psi}{3 \sqrt[3]{2} \cdot b}-\frac{\sqrt[3]{2}\left(-c^{2}+3 b+3 \gamma \cdot a \cdot b\right)}{3 b \cdot \psi}$

- $\lambda_{2}=-\frac{c}{3 b}-\frac{(1-\sqrt{3} i) \psi}{6 \sqrt[3]{2} \cdot b}+\frac{(1+\sqrt{3} i)\left(-c^{2}+3 b+3 \gamma \cdot a \cdot b\right)}{3 \sqrt[3]{4} \cdot b \cdot \psi}$

- $\lambda_{3}=-\frac{c}{3 b}+\frac{(1+\sqrt{3} i) \psi}{6 \sqrt[3]{2} \cdot b}+\frac{(1-\sqrt{3} i)\left(-c^{2}+3 b+3 \gamma \cdot a \cdot b\right)}{3 \sqrt[3]{4} \cdot b \cdot \psi}$

Nas expressões dos autovalores de (2.87), considera-se que:

- $\psi=\sqrt[3]{\eta+\xi}$

- $\quad \eta=-2 c^{3}+9 b \cdot c+9 \gamma \cdot a \cdot b \cdot c+27 \gamma \cdot b^{2}$

- $\xi=\sqrt{4\left(-c^{2}+3 \cdot b+3 \gamma \cdot a \cdot b\right)^{3}+\eta^{2}}$;

$\gamma=\mu\left[ \pm \sqrt{1-\left(\frac{\Omega}{\mu}\right)^{2}}\right]$

O próximo passo na análise linear da estabilidade trata da obtenção da parte real dos autovalores. Deve-se verificar para quais combinações de parâmetros $(a, b$, $c, \gamma)$ cada autovalor pode assumir parte real negativa, nula ou positiva. Observandose as expressões dos autovalores em (2.87), percebe-se que haveria uma dificuldade considerável em proceder a essa análise analiticamente. Mais uma vez, a alternativa encontrada foi simplificar o sistema dinâmico, transformando o parâmetro $\gamma \mathrm{em}$ variável de estado através da inclusão de uma nova equação diferencial sem dinâmica, ou seja, $\dot{\gamma}=0$. Antes, porém, algumas mudanças no sistema (2.61) serão feitas. Em primeiro lugar, faz-se a expansão em série de Taylor das funções seno e co-seno, em torno de $x_{1}^{*}=\arccos \left[ \pm \sqrt{1-\left(\frac{\Omega}{\mu}\right)^{2}}\right]$

$$
\begin{aligned}
& \operatorname{sen} x_{1} \approx \operatorname{sen} \rho+\cos \rho \cdot\left(x_{1}-\rho\right)-\frac{1}{2} \operatorname{sen} \rho \cdot\left(x_{1}-\rho\right)^{2}+\ldots \mathrm{e} \\
& \cos x_{1} \approx \cos \rho-\operatorname{sen} \rho \cdot\left(x_{1}-\rho\right)+\ldots
\end{aligned}
$$


Sendo que em (.+88), define-se $\rho=\arccos \left[ \pm \sqrt{1-\left(\frac{\Omega}{\mu}\right)^{2}}\right]$.

Em segundo lugar, faz-se a translação do sistema de coordenadas para a origem, considerando-se a seguinte mudança de variáveis:

$$
\bar{x}_{1}=x_{1}-\rho, \bar{x}_{2}=x_{2} \text { e } \bar{x}_{3}=x_{3}
$$

Então, o sistema (2.80), transladado para a origem, pode ser reescrito como segue:

$$
\left\{\begin{array}{l}
\dot{\bar{x}}_{1}=\bar{x}_{2} \\
\dot{\bar{x}}_{2}=\bar{x}_{3} \\
\dot{\bar{x}}_{3}=-\frac{c}{b} \bar{x}_{3}-\frac{1}{b} \bar{x}_{2}-\frac{a}{b} \gamma \cdot \bar{x}_{2}+\frac{\mu}{b} \Omega \cdot \bar{x}_{1} \cdot \bar{x}_{2}-\frac{1}{b} \gamma \cdot \bar{x}_{1}+\frac{1}{2 b} \Omega \cdot \bar{x}_{1}^{2}
\end{array}\right.
$$

Então, transforma-se o parâmetro $\gamma=\mu\left[ \pm \sqrt{1-\left(\frac{\Omega}{\mu}\right)^{2}}\right]$ em variável de estado.

Assim, o sistema (2.90) fica:

$$
\left\{\begin{array}{l}
\dot{\bar{x}}_{1}=\bar{x}_{2} \\
\dot{\bar{x}}_{2}=\bar{x}_{3} \\
\dot{\bar{x}}_{3}=-\frac{c}{b} \bar{x}_{3}-\frac{1}{b} \bar{x}_{2}-\frac{a}{b} \gamma \cdot \bar{x}_{2}+\frac{\mu}{b} \Omega \cdot \bar{x}_{1} \cdot \bar{x}_{2}-\frac{1}{b} \gamma \cdot \bar{x}_{1}+\frac{1}{2 b} \Omega \cdot \bar{x}_{1}^{2} \\
\mathrm{e} \quad \dot{\gamma}=0
\end{array}\right.
$$

Os pontos de equilíbrio de (2.91) podem ser obtidos como segue: 


$$
\left\{\begin{array}{l}
\dot{\bar{x}}_{1}=0 \Rightarrow \bar{x}_{3}^{*}=0 \\
\dot{\bar{x}}_{2}=0 \Rightarrow \bar{x}_{3}^{*}=0 \\
\dot{\bar{x}}_{3}=0 \Rightarrow \bar{x}_{1}^{*}=0
\end{array}\right.
$$

Por simplificação, omitem-se referências à nova variável $\gamma$. O novo ponto de equilíbrio, tanto para $\gamma=\mu\left[+\sqrt{1-\left(\frac{\Omega}{\mu}\right)^{2}}\right]$, quanto para $\gamma=\mu\left[-\sqrt{1-\left(\frac{\Omega}{\mu}\right)^{2}}\right]$, é $P=(0,0,0)$.

A matriz Jacobiana, calculada no ponto de equilíbrio $P=(0,0,0)$ de $(2.91)$, é:

$$
J_{P}=\left[\begin{array}{rrr}
0 & 1 & 0 \\
0 & 0 & 1 \\
0 & -\frac{1}{b} & -\frac{c}{b}
\end{array}\right]
$$

Assim, os autovalores de $J_{P}$ são:

- $\lambda_{1}=0$

$$
\begin{aligned}
&- \lambda_{2}=-\frac{\left(c+\sqrt{c^{2}-4 b}\right)}{2 b} \\
& \text { - } \lambda_{3}=\frac{\left(\sqrt{c^{2}-4 b}-c\right)}{2 b}
\end{aligned}
$$

Através da análise idêntica a ocorrida em (2.36) e (2.70), pode-se verificar que:

- O autovalor $\lambda_{1}$ é nulo, independentemente dos valores ou relações que possam assumir os parâmetros envolvidos. 
- Os autovalores $\lambda_{2}$ e $\lambda_{3}$ são ou reais com parte real negativa, para $c^{2} \geq 4 b$, ou pares de complexos conjugados com parte real negativa, para $c^{2}<4 b$.

Portanto, graças à existência de um autovalor nulo, não é possível obter conclusões sobre a estabilidade de $P$, através da análise linear. Para investigar o comportamento do sistema no subespaço central, será aplicado o Teorema da Variedade Central (TVC) na subseção seguinte.

\subsubsection{ANÁLISE DA ESTABILIDADE DE $P=(0,0,0)$ VIA TVC}

Primeiramente, reescreve-se o sistema (2.91) na forma matricial, como segue:

$$
\begin{aligned}
& {\left[\begin{array}{c}
\dot{\bar{x}}_{1} \\
\dot{\bar{x}}_{2} \\
\dot{\bar{x}}_{3}
\end{array}\right]=\left[\begin{array}{ccc}
0 & 1 & 0 \\
0 & 0 & 1 \\
0 & -\frac{1}{b} & -\frac{c}{b}
\end{array}\right]\left[\begin{array}{c}
\bar{x}_{1} \\
\bar{x}_{2} \\
\bar{x}_{3}
\end{array}\right]+\left[\begin{array}{c}
0 \\
0 \\
-\frac{a}{b} \gamma \cdot \bar{x}_{2}+\frac{\mu}{b} \Omega \cdot \bar{x}_{1} \cdot \bar{x}_{2}-\frac{1}{b} \gamma \cdot \bar{x}_{1}+\frac{1}{2 b} \Omega \cdot \bar{x}_{1}^{2}
\end{array}\right]} \\
& \text { e } \quad \dot{\gamma}=0
\end{aligned}
$$

A expressão (2.95) pode ser representada por $\dot{\bar{X}}=J_{P} \cdot \bar{X}+\bar{F}$ e $\dot{\gamma}=0$.

Como $\gamma=\mu\left[ \pm \sqrt{1-\left(\frac{\Omega}{\mu}\right)^{2}}\right]$, ou seja, $\gamma= \pm \sqrt{\mu^{2}-\Omega^{2}}$, pode-se definir o seguinte parâmetro k:

$$
k=\sqrt{m^{2}-1} \text {, sendo } \mu=m \cdot \Omega, \forall m \in \mathbb{R}
$$

Dessa forma, pode-se reescrever a expressão de $\gamma$ :

$$
\gamma= \pm \sqrt{\mu^{2}-\Omega^{2}}= \pm \Omega \sqrt{m^{2}-1}= \pm \Omega \cdot k
$$

Então, a nova variável a ser utilizada no sistema de equações no espaço de estados é $\Omega$, substituindo-se $\gamma$ por $\gamma= \pm \Omega \cdot k$ e a equação $\dot{\gamma}=0$ por $\dot{\Omega}=0$.

Conforme requerido pelo TVC, o sistema (2.95) precisa ser reescrito na forma canônica de Jordan. Para isso, define-se um novo conjunto de variáveis de estado $V=\left(v_{1}, v_{2}, v_{3}\right)^{T}$ de modo que $\bar{X}=M \cdot V$, sendo $\mathrm{M}$ uma base composta pelos 
autovetores de $J_{P}$. Assim, é possível dizer que $V=\left[M^{-1} \cdot J_{P_{1,2}} \cdot M\right] \cdot V+M^{-1} \cdot \bar{F} \mathrm{e}$ $\dot{\Omega}=0$, ou seja:

$$
\begin{aligned}
& {\left[\begin{array}{l}
\dot{v}_{1} \\
\dot{v}_{1} \\
\dot{v}_{3}
\end{array}\right]=\left[\begin{array}{ccc}
0 & 0 & 0 \\
0 & -\frac{\left(c_{i}+\sqrt{c_{i}^{2}-4 b_{i}}\right)}{2 b_{i}} & 0 \\
0 & 0 & \frac{\left(\sqrt{c_{i}^{2}-4 b_{i}}-c_{i}\right)}{2 b_{i}}
\end{array}\right] \cdot\left[\begin{array}{c}
v_{1} \\
v_{2} \\
v_{3}
\end{array}\right]+\left[\begin{array}{l}
\bar{f}_{1} \\
\overline{f_{2}} \\
\overline{f_{3}}
\end{array}\right]} \\
& \text { e } \dot{\Omega}=0
\end{aligned}
$$

Sendo que em (2.96), tem-se:

$$
\left[\begin{array}{l}
f_{1} \\
f_{2} \\
f_{3} \\
f_{4} \\
f_{5} \\
f_{6}
\end{array}\right]=M^{-1} \cdot \bar{F}
$$

Por questão de simplicidade, optou-se por omitir os termos não-lineares $f_{1}$, $f_{2}, f_{3}, f_{4}, f_{5}$ e $f_{6}$ devido a sua extensão.

Então, de acordo com o Teorema da Variedade Central (Carr, 1981; Guckenheimer e Holmes, 1983; Wiggins, 1990; Monteiro, 2002), procura-se a variedade central $W^{c}$ que seja tangente ao subespaço central $E^{c}$ no ponto de equilíbrio $P$. Assim, para $x$ e $\Omega$ suficientemente pequenos, tem-se:

$$
\begin{aligned}
W^{c}(0)=\{ & \left(v_{1}, v_{2}, v_{3}, \Omega\right) \in \mathfrak{R}^{4} / v_{1}=x, v_{2}=h_{1}(x, \Omega), v_{3}=h_{2}(x, \Omega), \\
& \left.h_{j}(0,0)=0, D h_{j}(0,0)=0, j=1,2\right\}
\end{aligned}
$$

Considere-se então, os seguintes polinômios estimadores $h_{1}(x, \Omega)$ e $h_{2}(x, \Omega)$, compostos por monômios do terceiro grau:

$$
\begin{cases}h_{1}(x, \Omega)=\alpha_{1} \cdot x^{2}+\alpha_{2} \cdot x \cdot \Omega+\alpha_{3} \cdot \Omega^{2}, & \left(\alpha_{1}, \alpha_{2}, \alpha_{3}\right) \in \mathfrak{R} \\ h_{2}(x, \Omega)=\beta_{1} \cdot x^{2}+\beta_{2} \cdot x \cdot \Omega+\beta_{3} \cdot \Omega^{2}, & \left(\beta_{1}, \beta_{2}, \beta_{3}\right) \in \Re\end{cases}
$$


Pelo Teorema de Carr (Carr, 1981; Guckenheimer e Holmes, 1983; Wiggins, 1990; Monteiro, 2002), a variedade central deve satisfazer $N(h(x, \Omega))=0$, ou seja:

$$
\left\{\begin{array}{r}
\frac{\partial h_{1}(x, \Omega)}{\partial x}\left[\lambda_{1} \cdot x+\bar{f}_{1}\left(x, h_{1}(x, \Omega), h_{2}(x, \Omega), \Omega\right)\right]- \\
\quad-\lambda_{2} \cdot h_{2}(x, \Omega)-\bar{f}_{2}\left(x, h_{1}(x, \Omega), h_{2}(x, \Omega), \Omega\right)=0 \\
\\
\begin{array}{rl}
\frac{\partial h_{2}(x, \Omega)}{\partial x}\left[\lambda_{1} \cdot x+\bar{f}_{1}\left(x, h_{1}(x, \Omega), h_{2}(x, \Omega), \Omega\right)\right]- \\
-\lambda_{3} \cdot h_{2}(x, \Omega)-\bar{f}_{3}\left(x, h_{1}(x, \Omega), h_{2}(x, \Omega), \Omega\right)=0
\end{array}
\end{array}\right.
$$

Utilizando (2.96) e (2.98) em (2.99), tem-se:

$$
\begin{aligned}
& \left(2 \alpha_{1} \cdot x+\alpha_{2} \cdot x \cdot \gamma\right) \cdot\left[0+\bar{f}_{1}\right]-\lambda_{2} \cdot\left(\alpha_{1} \cdot x^{2}+\alpha_{2} \cdot x \cdot \Omega+\alpha_{3} \cdot \Omega^{2}\right)-\bar{f}_{2}=0 \\
& \left(2 \beta_{1} \cdot x+\beta_{2} \cdot x \cdot \Omega\right) \cdot\left[0+\bar{f}_{1}\right]-\lambda_{3} \cdot\left(\beta_{1} \cdot x^{2}+\beta_{2} \cdot x \cdot \Omega+\beta_{3} \cdot \Omega^{2}\right)-\bar{f}_{3}=0
\end{aligned}
$$

Pela resolução de (2.100) igualando-se os termos de igual potência, os coeficientes de $h_{1}(x, \Omega)$ e $h_{2}(x, \Omega)$ :

$$
\begin{aligned}
& \left\{\begin{array}{l}
x^{2}: \quad \alpha_{1}=0 \\
\Omega \cdot x: \quad \alpha_{2}= \pm \frac{\sqrt{c^{2}-4 b}}{4 b-c^{2}} k \Rightarrow h_{1}(x, \Omega)= \pm \frac{\sqrt{c^{2}-4 b}}{4 b-c^{2}} k \cdot \Omega \cdot x \\
\Omega^{2}: \quad \alpha_{3}=0
\end{array}\right. \\
& \left\{\begin{array}{l}
x^{2}: \quad \beta_{1}=0 \\
\Omega \cdot x: \quad \beta_{2}= \pm \frac{\sqrt{c^{2}-4 b}}{c^{2}-4 b} k \Rightarrow h_{2}(x, \gamma)= \pm \frac{\sqrt{c^{2}-4 b}}{c^{2}-4 b} k \cdot \Omega \cdot x \\
\Omega^{2}: \quad \beta_{3}=0
\end{array}\right.
\end{aligned}
$$

Substituindo (2.101) e (2.102) no sistema (2.96) pode-se obter, desprezando os termos de ordem superior, o campo de vetores restrito à variedade central: 


$$
\left\{\begin{array}{l}
\dot{x}=\frac{1}{2} x \cdot \Omega\left(x+k\left(c(c-2 a) k \cdot x \cdot \Omega^{2}+2(a-c)(k \mp x) \Omega \mp 2\right)\right) \\
\dot{\Omega}=0
\end{array}\right.
$$

Para obter os pontos de equilíbrio de (2.103), basta fazer:

$$
\dot{x}=0 \Rightarrow x_{1}^{*}=0 \text { e } x_{2}{ }^{*}=\frac{k}{(c-2 a) k \cdot \Omega \pm 1}+\frac{k}{c \cdot k \cdot \Omega \pm 1}
$$

Verifica-se as estabilidades dos pontos de equilíbrio de (2.104) derivando parcialmente (2.103) em relação à $x$ :

$$
\frac{\partial(\dot{x})}{\partial x}=\Omega\left(c(c-2 a) k^{2} \cdot x \cdot \Omega^{2}+(a-c)(k \mp 2 x) \Omega \mp k+x\right)
$$

Portanto:

$$
\begin{aligned}
& \left.\frac{\partial(\dot{x})}{\partial x}\right|_{x_{1}^{*}}=k \cdot \Omega((a-c) k \cdot \Omega \mp 1) \\
& \left.\frac{\partial(\dot{x})}{\partial x}\right|_{x_{2,3}^{*}}= \pm k \cdot \Omega((a-c) k \cdot \Omega+1)
\end{aligned}
$$

De (2.106) conclui-se que:

- $\quad \operatorname{Em} x_{1}^{*}$

Para $\gamma=+\Omega \cdot k$, tem-se que $\operatorname{Re}\left[\left.\frac{\partial(\dot{x})}{\partial x}\right|_{x_{1}^{*}}\right]<0 \Leftrightarrow$

$$
\begin{aligned}
& \left(\Omega<0 \wedge c>a \wedge k>\frac{1}{(a-c) \Omega}\right) \vee \\
& \vee\left(\Omega>0 \wedge\left(\left(c<a \wedge 0<k<\frac{1}{(a-c) \Omega}\right) \vee(c \geq a \wedge k>0)\right)\right.
\end{aligned}
$$

Para $\gamma=+\Omega \cdot k$, tem-se que $\operatorname{Re}\left[\left.\frac{\partial(\dot{x})}{\partial x}\right|_{x_{1}^{*}}\right]>0 \Leftrightarrow$ 


$$
\begin{aligned}
& \left(\Omega<0 \wedge\left((c \leq a \wedge k>0) \vee\left(c>a \wedge 0<k<\frac{1}{(a-c) \Omega}\right)\right)\right) \vee \\
& \vee\left(\Omega>0 \wedge c<a \wedge k>\frac{1}{(a-c) \Omega}\right)
\end{aligned}
$$

Para $\gamma=-\Omega \cdot k$, tem-se que $\operatorname{Re}\left[\left.\frac{\partial(\dot{x})}{\partial x}\right|_{x_{1}^{*}}\right]<0 \Leftrightarrow$

$$
\begin{aligned}
& \left(\Omega<0 \wedge\left(\left(c<a \wedge 0<k<-\frac{1}{(a-c) \Omega}\right) \vee(c \geq a \wedge k>0)\right)\right) \vee \\
& \left(\Omega<0 \wedge c>a \wedge k>-\frac{1}{(a-c) \Omega}\right) \vee
\end{aligned}
$$

Para $\gamma=-\Omega \cdot k$, tem-se que $\operatorname{Re}\left[\left.\frac{\partial(\dot{x})}{\partial x}\right|_{x_{1}^{*}}\right]>0 \Leftrightarrow$

$$
\begin{aligned}
& \left(\Omega<0 \wedge c<a \wedge k>-\frac{1}{(a-c) \Omega}\right) \vee \\
& \vee\left(\Omega>0 \wedge\left((c \leq a \wedge k>0) \vee\left(c>a \wedge 0<k<-\frac{1}{(a-c) \Omega}\right)\right)\right)
\end{aligned}
$$

- $\operatorname{Em} x_{2}{ }^{*}$

Para $\gamma=+\Omega \cdot k$, tem-se que $\operatorname{Re}\left[\left.\frac{\partial(\dot{x})}{\partial x}\right|_{x_{2}^{*}}\right]<0 \Leftrightarrow$

$$
\begin{aligned}
& \left(\Omega<0 \wedge\left((c \leq a \wedge k>0) \vee\left(c>a \wedge 0<k<\frac{1}{(a-c) \Omega}\right)\right)\right) \vee \\
& \vee\left(\Omega>0 \wedge c<a \wedge k>\frac{1}{(a-c) \Omega}\right)
\end{aligned}
$$


Para $\gamma=+\Omega \cdot k$, tem-se que $\operatorname{Re}\left[\left.\frac{\partial(\dot{x})}{\partial x}\right|_{x_{1}^{*}}\right]>0 \Leftrightarrow$

$$
\begin{aligned}
& \left(\Omega<0 \wedge c>a \wedge k>\frac{1}{(a-c) \Omega}\right) \vee \\
& \vee\left(\Omega>0 \wedge\left(\left(c<a \wedge 0<k<\frac{1}{(a-c) \Omega}\right) \vee(c \geq a \wedge k>0)\right)\right)
\end{aligned}
$$

Para $\gamma=-\Omega \cdot k$, tem-se que $\operatorname{Re}\left[\left.\frac{\partial(\dot{x})}{\partial x}\right|_{x_{2}^{*}}\right]<0 \Leftrightarrow$

$$
\begin{aligned}
& \left(\Omega<0 \wedge c<a \wedge k>-\frac{1}{(a-c) \Omega}\right) \vee \\
& \vee\left(\Omega>0 \wedge\left((c \leq a \wedge k>0) \vee\left(c>a \wedge 0<k<-\frac{1}{(a-c) \Omega}\right)\right)\right)
\end{aligned}
$$

Para $\gamma=-\Omega \cdot k$, tem-se que $\operatorname{Re}\left[\left.\frac{\partial(\dot{x})}{\partial x}\right|_{x_{1}^{*}}\right]>0 \Leftrightarrow$

$$
\begin{aligned}
& \left(\Omega<0 \wedge\left(\left(c<a \wedge 0<k<-\frac{1}{(a-c) \Omega}\right) \vee(c \geq a \wedge k>0)\right)\right) \vee \\
& \left(\Omega>0 \wedge c>a \wedge k>-\frac{1}{(a-c) \Omega}\right) \vee
\end{aligned}
$$

No capítulo VI serão realizadas as devidas conclusões a respeito das expressões obtidas em (2.107) e (2.108), que tratam da estabilidade dos pontos de (2.104).

No próximo capítulo, o estudo realizado no capítulo II é estendido para um PLL de uma rede OWMS Estrela - Cadeia Simples. 


\section{CAPÍTULO III}

\section{SISTEMA DINÂMICO DE UM PLL DE $3^{\text {a }}$ ORDEM DE UMA REDE OWMS ESTRELA - CADEIA SIMPLES.}

No capítulo II foi realizado o estudo de um PLL de $3^{\mathrm{a}}$ ordem isolado, isto é, sem a preocupação com a origem do sinal de entrada. Entretanto, para PLLs que compõem um nó escravo de uma rede OWMS Estrela-Cadeia Simples, este sinal tanto pode ser proveniente do mestre como de outro escravo. Por isso, o objetivo deste capítulo é realizar um estudo análogo àquele do capítulo II, segundo a consideração que o PLL em análise ocupa uma posição específica em uma rede OWMS Estrela - Cadeia Simples. Na primeira seção, os escravos serão classificados segundo a posição que ocupam na rede. Nas seções seguintes serão obtidas as equações ou sistema de equações que descrevem o sincronismo de um escravo qualquer.

\subsection{CLASSIFICAÇÃO DOS ESCRAVOS QUANTO AO NÍVEL DE PROXIMIDADE EM RELAÇÃO AO MESTRE}

Nesta seção, a proposta é classificar um nó escravo da rede segundo o número de conexões existentes entre este nó e o mestre. Então, serão assim denominados escravos do nível I, àqueles que estejam diretamente conectados ao nó mestre, ou seja, quando há somente uma conexão entre estes dois nós. Qualquer nó da rede que esteja conectado a um escravo do nível I será denominado escravo do nível II, uma vez que haverá duas conexões entre este e o mestre. A figura 3.1 ilustra a idéia:

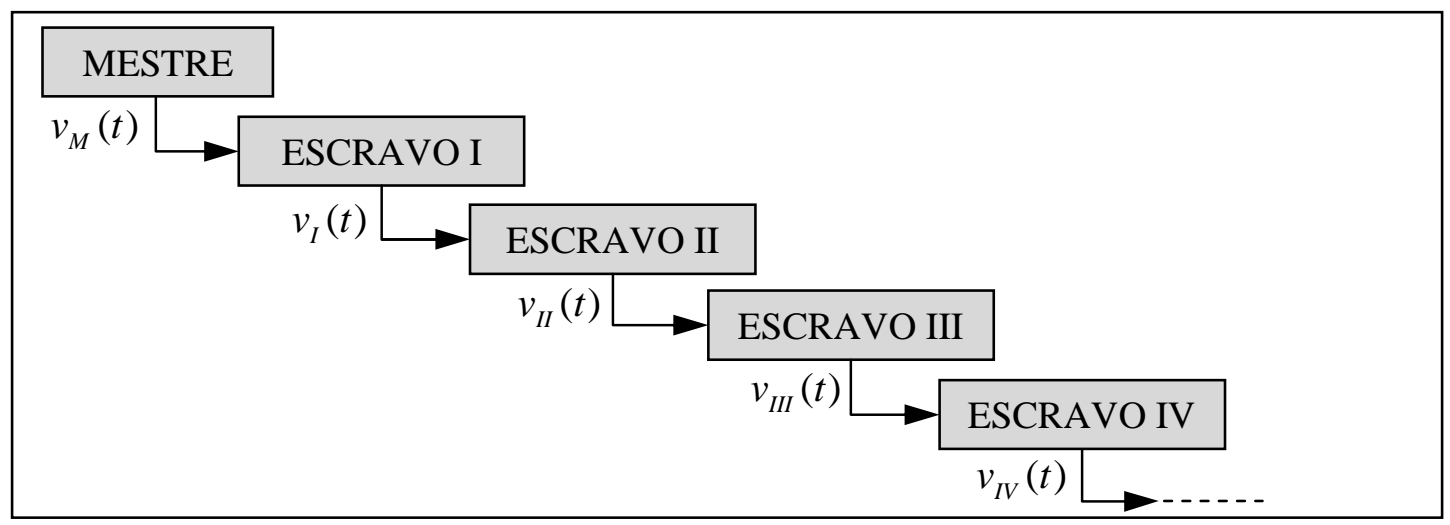

Fig. 3.1: Esquema simplificado dos níveis de proximidade dos escravos em relação ao mestre em redes OWMS Estrela - Cadeia Simples. 
Nesse esquema estão definidas as mesmas grandezas físicas da seção 2.1 do capítulo II, particularizadas para cada nó segundo seu índice. Assim, para o esquema da figura (3.1), considera-se que $v_{M}(t)$ é o sinal de sai do mestre com destino a um escravo I, $v_{I}(t)$ é o sinal de sai de um escravo I com destino a um escravo II, e assim por diante.

A seguir, será obtida a equação que descreve a dinâmica de um escravo do nível I.

\subsection{EQUAÇÃO DINÂMICA DE UM ESCRAVO DO NÍVEL I}

O sinal proveniente do mestre é considerado periódico, sendo descrito pela seguinte equação:

$$
v_{M}(t)=V_{M} \cdot \operatorname{sen} \Phi_{M}(t)=V_{M} \cdot \operatorname{sen}\left[\omega_{0} \cdot t+\theta_{M}(t)\right]
$$

Em (3.1), tem-se que:

- $V_{M}$ é a amplitude do sinal do mestre, suposta constante e positiva;

- $\Phi_{M}(t)$ é a fase do sinal do mestre;

- $\omega_{0}$ é a freqüência angular central do sinal do mestre, suposta constante e positiva;

- $\theta_{M}(t)$ é a mudança de fase do sinal do mestre.

O sinal gerado pelo VCO do escravo do nível I também é do tipo periódico, sendo descrito pela seguinte equação:

$$
v_{I}(t)=V_{I} \cdot \cos \Phi_{I}(t)=V_{I} \cdot \cos \left[\omega_{0} \cdot t+\theta_{I}(t)\right]
$$

Relembrando que em (3.2), tem-se:

- $V_{I}$ é a amplitude do sinal do escravo do nível I, suposta constante e positiva;

- $\Phi_{I}(t)$ é a fase do sinal do escravo do nível I;

- $\omega_{0}$ é a frequiência angular central do sinal do escravo do nível I, suposta constante, positiva e idêntica à do sinal do mestre;

- $\theta_{I}(t)$ é a mudança de fase do sinal do escravo do nível I. 
Assumindo as mesmas hipóteses do capítulo 2, seção 2.1, pode-se obter a expressão do erro de fase dinâmico relativo ao escravo do nível I:

$$
v_{d, I}(t)=\frac{K_{m, I} \cdot V_{M} \cdot V_{I}}{2} \cdot \operatorname{sen}\left(\theta_{M}(t)-\theta_{I}(t)\right)
$$

Sendo que em (3.3), $K_{m, I}$ é o ganho do PD do escravo do nível I.

Para um escravo I, considera-se um LPF com a seguinte função de transferência:

$$
F_{I}(s)=\frac{V_{c, I}(s)}{V_{d, I}(s)}=\frac{a_{I} \cdot s+1}{b_{I} \cdot s^{2}+c_{I} \cdot s+1}
$$

Ainda, a equação que descreve a dinâmica do VCO deste escravo é dada por:

$$
v_{c, I}(t)=\frac{\dot{\theta}_{I}(t)}{K_{I}}
$$

Sendo que em (3.5), $K_{I}$ é o ganho do VCO do escravo do nível I.

Para descrever a estabilidade do nó considerado, define-se o seguinte o erro de fase:

$$
\varphi_{M I}(t) \stackrel{\Delta}{=} \Phi_{M}(t)-\Phi_{I}(t), \quad \varphi_{M I}(t) \in[-\pi, \pi[
$$

Assumindo as mesmas hipóteses do capítulo II, seção 2.1, é possível escrever a equação que descreve a dinâmica do PLL considerado como:

$$
\begin{gathered}
b_{I} \cdot \dddot{\varphi}_{M, I}(t)+c_{I} \cdot \ddot{\varphi}_{M, I}(t)+\dot{\varphi}_{M, I}(t)+a_{I} \cdot \mu_{I} \cdot \dot{\varphi}_{M, I}(t) \cdot \cos \varphi_{M, I}(t)+\mu_{I} \cdot \operatorname{sen} \varphi_{M, I}(t)= \\
=b_{I} \cdot \dddot{\theta}_{M}(t)+c_{I} \cdot \ddot{\theta}_{M}(t)+\dot{\theta}_{M}(t)
\end{gathered}
$$

Sendo que em (3.7), o seguinte parâmetro foi definido:

$$
\mu_{I} \stackrel{\Delta}{=} \frac{K_{I} \cdot K_{m, I} \cdot V_{M} \cdot V_{I}}{2}
$$

Note-se que a equação (3.7) é idêntica, a menos dos índices, à equação (2.27) que foi obtida no estudo de um PLL isolado. Logo, todos os resultados para um escravo do nível I são idênticos àqueles que já foram apresentados no capítulo 2, ou seja, não é necessário proceder ao seu estudo.

A seguir, será obtida a equação que descreve a dinâmica de um escravo do nível II. 


\subsection{EQUAÇÃO DINÂMICA DE UM ESCRAVO DO NÍVEL II}

Em um escravo do nível II, o sinal de entrada $v_{I}(t)$ é proveniente do VCO de um escravo do nível I, isto é, diretamente conectado ao mestre. Este sinal é considerado periódico, sendo descrito pela equação (3.2).

$\mathrm{O}$ sinal que é gerado pelo VCO do escravo II, $v_{I I}(t)$, também é considerado periódico. Ele pode descrito pela seguinte equação:

$$
v_{I I}(t)=-V_{I I} \cdot \operatorname{sen} \Phi_{I I}(t)=-V_{I I} \cdot \operatorname{sen}\left[\omega_{0} \cdot t+\theta_{I I}(t)\right]
$$

Em que:

- $\quad V_{I I}$ é a amplitude do sinal, constante e positiva;

- $\Phi_{I I}(t)$ é a fase do sinal, variável no tempo;

- $\omega_{0}$ é a freqüência angular central do sinal, suposta constante, positiva e aqui considerada como sendo idêntica à do sinal de entrada $v_{I}(t)$;

- $\theta_{I I}(t)$ é a mudança de fase, variável no tempo.

É importante destacar que toda comparação de sinais em um determinado PD é realizada para sinais em quadratura. Desse modo, como o sinal $v_{I}(t)$ em (3.2) é descrito como função do cosseno da sua fase, o sinal $v_{I I}(t)$ em (3.9) é descrito como função do seno da sua fase, mas com sinal negativo. Sendo assim, pode-se escrever a saída do PD do escravo II como sendo:

$$
v_{d, I I}(t)=K_{m, I I} \cdot v_{I}(t) \cdot v_{I I}(t)
$$

Sendo que em (3.10), $K_{m, I I}$ é o ganho do PD.

Desse modo, substituindo (3.2) e (3.9) em (3.10), tem-se:

$$
v_{d, I I}(t)=-K_{m, I I} \cdot V_{I} \cdot \cos \left[\omega_{0} \cdot t+\theta_{I}(t)\right] \cdot V_{I I} \cdot \operatorname{sen}\left[\omega_{0} \cdot t+\theta_{I I}(t)\right]
$$

Desenvolvendo os termos trigonométricos da equação (3.11), tem-se:

$$
\begin{gathered}
v_{d, I I}(t)=-K_{m, I I} \cdot V_{I} \cdot\left[\cos \left(\omega_{0} \cdot t\right) \cdot \cos \theta_{I}(t)-\operatorname{sen}\left(\omega_{0} \cdot t\right) \cdot \operatorname{sen} \theta_{I}(t)\right] \\
\cdot V_{I I} \cdot\left[\operatorname{sen}\left(\omega_{0} \cdot t\right) \cdot \cos \theta_{I I}(t)+\cos \left(\omega_{0} \cdot t\right) \cdot \operatorname{sen} \theta_{I I}(t)\right]
\end{gathered}
$$

Multiplicando os fatores de (3.12) que estão entre colchetes, tem-se: 


$$
\begin{aligned}
v_{d, I I}(t)=-K_{m, I I} \cdot & V_{I} \cdot V_{I I} \cdot\left[\operatorname{sen}\left(\omega_{0} \cdot t\right) \cdot \cos \theta_{I I}(t) \cdot \cos \left(\omega_{0} \cdot t\right) \cdot \cos \theta_{I}(t)+\right. \\
& +\cos \left(\omega_{0} \cdot t\right) \cdot \operatorname{sen} \theta_{I I}(t) \cdot \cos \left(\omega_{0} \cdot t\right) \cdot \cos \theta_{I}(t)- \\
& -\operatorname{sen}\left(\omega_{0} \cdot t\right) \cdot \cos \theta_{I I}(t) \cdot \operatorname{sen}\left(\omega_{0} \cdot t\right) \cdot \operatorname{sen} \theta_{I}(t)- \\
& \left.-\cos \left(\omega_{0} \cdot t\right) \cdot \operatorname{sen} \theta_{I I}(t) \cdot \operatorname{sen}\left(\omega_{0} \cdot t\right) \cdot \operatorname{sen} \theta_{I}(t)\right]
\end{aligned}
$$

Ou seja:

$$
\begin{aligned}
& v_{d, I I}(t)=-K_{m, I I} \cdot V_{I} \cdot V_{I I} \cdot\left[\operatorname{sen}\left(\omega_{0} \cdot t\right) \cdot \cos \left(\omega_{0} \cdot t\right) \cdot \cos \theta_{I}(t) \cdot \cos \theta_{I I}(t)+\right. \\
&+ \cos ^{2}\left(\omega_{0} \cdot t\right) \cdot \operatorname{sen} \theta_{I I}(t) \cdot \cos \theta_{I}(t)- \\
&-\operatorname{sen}^{2}\left(\omega_{0} \cdot t\right) \cdot \cos \theta_{I I}(t) \cdot \operatorname{sen} \theta_{I}(t)- \\
&\left.-\operatorname{sen}\left(\omega_{0} \cdot t\right) \cdot \cos \left(\omega_{0} \cdot t\right) \cdot \operatorname{sen} \theta_{I}(t) \cdot \operatorname{sen} \theta_{I I}(t)\right]
\end{aligned}
$$

Então, fazendo as devidas transformações trigonométricas em (3.14), tem-se:

$$
\begin{aligned}
& v_{d, I I}(t)=-K_{m, I I} \cdot V_{I} \cdot V_{I I} \cdot\left\{\frac{\operatorname{sen}\left(2 \omega_{0} \cdot t\right)}{2} \cdot \cos \theta_{I}(t) \cdot \cos \theta_{I I}(t)+\right. \\
&+\left[\frac{1}{2}+\frac{1}{2} \cos \left(2 \omega_{0} \cdot t\right)\right] \cdot \operatorname{sen} \theta_{I I}(t) \cdot \cos \theta_{I}(t)- \\
&-\left[\frac{1}{2}-\frac{1}{2} \cos \left(2 \omega_{0} \cdot t\right)\right] \cdot \cos \theta_{I I}(t) \cdot \operatorname{sen} \theta_{I}(t)- \\
&\left.-\frac{\operatorname{sen}\left(2 \omega_{0} \cdot t\right)}{2} \cdot \operatorname{sen} \theta_{I}(t) \cdot \operatorname{sen} \theta_{I I}(t)\right\}
\end{aligned}
$$

Multiplicando os fatores de (3.15) que estão entre colchetes, tem-se:

$$
\begin{aligned}
v_{d, I I}(t)=-\frac{K_{m, I I} \cdot V_{I} \cdot V_{I I}}{2} \cdot\left[\operatorname{sen}\left(2 \omega_{0} \cdot t\right) \cdot \cos \theta_{I}(t) \cdot \cos \theta_{I I}(t)+\right. \\
\quad+\operatorname{sen} \theta_{I I}(t) \cdot \cos \theta_{I}(t)+\cos \left(2 \omega_{0} \cdot t\right) \cdot \operatorname{sen} \theta_{I I}(t) \cdot \cos \theta_{I}(t)- \\
\quad-\cos \theta_{I I}(t) \cdot \operatorname{sen} \theta_{I}(t)+\cos \left(2 \omega_{0} \cdot t\right) \cdot \cos \theta_{I I}(t) \cdot \operatorname{sen} \theta_{I}(t)- \\
\left.\quad-\operatorname{sen}\left(2 \omega_{0} \cdot t\right) \cdot \operatorname{sen} \theta_{I}(t) \cdot \operatorname{sen} \theta_{I I}(t)\right]
\end{aligned}
$$

Simplificando (3.16), tem-se: 


$$
\begin{aligned}
v_{d, I I}(t)=-\frac{K_{m, I I} \cdot V_{I} \cdot V_{I I}}{2} \cdot\left[\operatorname{sen}\left(2 \omega_{0} \cdot t\right) \cdot\left(\cos \theta_{I}(t) \cdot \cos \theta_{I I}(t)-\operatorname{sen} \theta_{I}(t) \cdot \operatorname{sen} \theta_{I I}(t)\right)\right. & +\cos \left(2 \omega_{0} \cdot t\right) \cdot\left(\operatorname{sen} \theta_{I I}(t) \cdot \cos \theta_{I}(t)+\cos \theta_{I I}(t) \cdot \operatorname{sen} \theta_{I}(t)\right) \\
& \left.+\operatorname{sen} \theta_{I I}(t) \cdot \cos \theta_{I}(t)-\cos \theta_{I I}(t) \cdot \operatorname{sen} \theta_{I}(t)\right]
\end{aligned}
$$

Assumindo-se que os termos de freqüência dupla de (3.17) podem ser eliminados pelo LPF, segue que:

$$
v_{d, I I}(t)=-\frac{K_{m, I I} \cdot V_{I} \cdot V_{I I}}{2} \cdot \operatorname{sen}\left(\theta_{I I}(t)-\theta_{I}(t)\right)=\frac{K_{m, I I} \cdot V_{I} \cdot V_{I I}}{2} \cdot \operatorname{sen}\left(\theta_{I}(t)-\theta_{I I}(t)\right)
$$

Para um escravo II, considera-se um LPF com a seguinte função de transferência:

$$
F_{I I}(s)=\frac{V_{c, I I}(s)}{V_{d, I I}(s)}=\frac{a_{I I} \cdot s+1}{b_{I I} \cdot s^{2}+c_{I I} \cdot s+1}
$$

Ainda, a equação que descreve a dinâmica do VCO deste escravo é dada por:

$$
v_{c, I I}(t)=\frac{\dot{\theta}_{I I}(t)}{K_{I I}}
$$

Sendo que em (3.20), $K_{I I}$ é o ganho do VCO do escravo do nível II.

Para descrever a estabilidade do nó considerado, define-se o seguinte o erro de fase:

$$
\varphi_{I, I I}(t) \stackrel{\Delta}{=} \Phi_{I}(t)-\Phi_{I I}(t), \quad \varphi_{I, I I}(t) \in[-\pi, \pi[
$$

Assumindo as mesmas hipóteses da subseção anterior, é possível escrever a equação que descreve a dinâmica do PLL considerado como sendo:

$$
\begin{gathered}
b_{I I} \cdot \dddot{\varphi}_{I, I I}(t)+c_{I I} \cdot \ddot{\varphi}_{I, I I}(t)+\dot{\varphi}_{I, I I}(t)+a_{I I} \cdot \mu_{I I} \cdot \dot{\varphi}_{I, I I}(t) \cdot \cos \varphi_{I, I I}(t)+\mu_{I I} \cdot \operatorname{sen} \varphi_{I, I I}(t)= \\
=b_{I I} \cdot \dddot{\theta}_{I}(t)+c_{I I} \cdot \ddot{\theta}_{I}(t)+\dot{\theta}_{I}(t)
\end{gathered}
$$

Sendo que em (3.22), o seguinte parâmetro foi definido:

$$
\mu_{I I} \stackrel{\Delta}{=} \frac{K_{I I} \cdot K_{m, I I} \cdot V_{I} \cdot V_{I I}}{2}
$$

A seguir, será obtida a equação que descreve a dinâmica de um escravo do nível III. 


\subsection{EQUAÇÃO DINÂMICA DE UM ESCRAVO DO NÍVEL III}

Em um escravo do nível III, o sinal de entrada $v_{I I}(t)$ é proveniente do VCO de um escravo do nível II. Este sinal é considerado periódico, sendo descrito pela equação (3.9).

$\mathrm{O}$ sinal que gerado pelo $\mathrm{VCO}$ do escravo III, $v_{I I I}(t)$, também é considerado periódico. Ele pode ser descrito pela seguinte equação:

$$
v_{I I I}(t)=-V_{I I I} \cdot \cos \Phi_{I I I}(t)=-V_{I I I} \cdot \cos \left[\omega_{0} \cdot t+\theta_{I I I}(t)\right]
$$

Em que:

- $V_{I I I}$ é a amplitude do sinal, constante e positiva;

- $\Phi_{I I I}(t)$ é a fase do sinal, variável no tempo;

- $\omega_{0}$ é a freqüência angular central do sinal, suposta constante, positiva e aqui considerada como sendo idêntica à do sinal de entrada $v_{I I}(t)$;

- $\theta_{I I I}(t)$ é a mudança de fase, variável no tempo.

Como na subseção anterior, destaca-se que a comparação de sinais no PD é realizada para sinais em quadratura. Desse modo, como o sinal $v_{I I}(t)$ em (3.9) é descrito como uma função do seno da sua fase, mas com sinal negativo, o sinal $v_{I I I}(t)$ em (3.24) é descrito como uma função do cosseno da sua fase e também com sinal negativo. Sendo assim, pode-se escrever a saída do PD do escravo III como sendo:

$$
v_{d, I I I}(t)=K_{m, I I I} \cdot v_{I I}(t) \cdot v_{I I I}(t)
$$

Sendo que em (3.25), $K_{m, I I I}$ é o ganho do PD.

Desse modo, substituindo (3.9) e (3.24) em (3.25), tem-se:

$$
v_{d, I I I}(t)=K_{m, I I I} \cdot V_{I I} \cdot \operatorname{sen}\left[\omega_{0} \cdot t+\theta_{I I}(t)\right] \cdot V_{I I I} \cdot \cos \left[\omega_{0} \cdot t+\theta_{I I I}(t)\right]
$$

O desenvolvimento da equação (3.26) é análogo ao da subseção 3.1.1. Assim sendo, desprezando-se os termos de freqüência dupla de (3.26), segue que:

$$
v_{d, I I I}(t)=\frac{K_{m, I I I} \cdot V_{I I} \cdot V_{I I I}}{2} \cdot \operatorname{sen}\left(\theta_{I I}(t)-\theta_{I I I}(t)\right)
$$

Para um escravo III, considera-se um LPF com a seguinte função de transferência: 


$$
F_{I I I}(s)=\frac{V_{c, I I I}(s)}{V_{d, I I I}(s)}=\frac{a_{I I I} \cdot s+1}{b_{I I I} \cdot s^{2}+c_{I I I} \cdot s+1}
$$

Ainda, a equação que descreve a dinâmica do VCO deste escravo é dada por:

$$
v_{c, I I I}(t)=\frac{\dot{\theta}_{I I I}(t)}{K_{I I I}}
$$

Sendo que em (3.29), $K_{I I I}$ é o ganho do VCO do escravo do nível III.

Para descrever a estabilidade do nó considerado, define-se o erro de fase:

$$
\varphi_{I I, I I I}(t) \stackrel{\Delta}{=} \Phi_{I I}(t)-\Phi_{I I I}(t), \quad \varphi_{I I, I I I}(t) \in[-\pi, \pi[
$$

Assumindo as mesmas hipóteses da subseção anterior, é possível escrever a equação que descreve a dinâmica do PLL considerado como sendo:

$$
\begin{aligned}
& b_{I I I} \cdot \dddot{\varphi}_{I I, I I I}(t)+c_{I I I} \cdot \ddot{\varphi}_{I I, I I I}(t)+\dot{\varphi}_{I I, I I}(t)+a_{I I I} \cdot \mu_{I I I} \cdot \dot{\varphi}_{I I, I I I}(t) \cdot \cos \varphi_{I I, I I I}(t)+ \\
& +\mu_{I I I} \cdot \operatorname{sen} \varphi_{I I, I I I}(t)=b_{I I I} \cdot \dddot{\theta}_{I I}(t)+c_{I I I} \cdot \ddot{\theta}_{I I}(t)+\dot{\theta}_{I I}(t)
\end{aligned}
$$

Sendo que em (3.31), o seguinte parâmetro foi definido:

$$
\mu_{I I I} \stackrel{\Delta}{=} \frac{K_{I I I} \cdot K_{m, I I I} \cdot V_{I I} \cdot V_{I I I}}{2}
$$

A seguir, será obtida a equação que descreve a dinâmica de um escravo do nível IV.

\subsection{EQUAÇÃO DINÂMICA DE UM ESCRAVO DO NÍVEL IV}

Em um escravo do nível IV, o sinal de entrada $v_{I I I}(t)$ é proveniente do VCO de um escravo do nível III. Este sinal é considerado periódico, sendo descrito pela equação (3.24).

$\mathrm{O}$ sinal que gerado pelo $\mathrm{VCO}$ do escravo IV, $v_{I V}(t)$, também é considerado periódico. Ele será descrito pela seguinte equação:

$$
v_{I V}(t)=V_{I V} \cdot \operatorname{sen} \Phi_{I V}(t)=V_{I V} \cdot \operatorname{sen}\left[\omega_{0} \cdot t+\theta_{I V}(t)\right]
$$

Em que:

- $\quad V_{I V}$ é a amplitude do sinal, constante e positiva;

- $\Phi_{I V}(t)$ é a fase do sinal, variável no tempo; 
- $\omega_{0}$ é a frequiência angular central do sinal, suposta constante, positiva e aqui considerada como sendo idêntica à do sinal de entrada $v_{I I I}(t)$;

- $\theta_{I V}(t)$ é a mudança de fase, variável no tempo.

Mais uma vez, como o sinal $v_{I I I}(t)$ em (3.24) é descrito como função do cosseno da sua fase, mas com sinal negativo, o sinal $v_{I V}(t)$ em (3.33) é descrito como função do seno da sua fase. Sendo assim, pode-se escrever a saída do PD do escravo IV como sendo:

$$
v_{d, I V}(t)=K_{m, I V} \cdot v_{I I I}(t) \cdot v_{I V}(t)
$$

Sendo que em (3.34), $K_{m, I I}$ é o ganho do PD.

Desse modo, substituindo (3.24) e (3.33) em (3.34), tem-se:

$$
v_{d, I V}(t)=-K_{m, I V} \cdot V_{I I I} \cdot \cos \left[\omega_{0} \cdot t+\theta_{I I I}(t)\right] \cdot V_{I V} \cdot \operatorname{sen}\left[\omega_{0} \cdot t+\theta_{I V}(t)\right]
$$

O desenvolvimento da equação (3.35) é análogo ao da subseção 3.1.2. Assim, desprezando-se os termos de freqüência dupla de (3.35), segue que:

$$
\begin{aligned}
v_{d, I V}(t)=-\frac{K_{m, I V} \cdot V_{I I I} \cdot V_{I V}}{2} \cdot \operatorname{sen}\left(\theta_{I I I}(t)-\theta_{I V}(t)\right)= \\
=\frac{K_{m, I V} \cdot V_{I I I} \cdot V_{I V}}{2} \cdot \operatorname{sen}\left(\theta_{I I I}(t)-\theta_{I V}(t)\right)
\end{aligned}
$$

Para um escravo IV, considera-se um LPF com a seguinte função de transferência:

$$
F_{I V}(s)=\frac{V_{c, I V}(s)}{V_{d, I V}(s)}=\frac{a_{I V} \cdot s+1}{b_{I V} \cdot s^{2}+c_{I V} \cdot s+1}
$$

Ainda, a equação que descreve a dinâmica do VCO deste escravo é dada por:

$$
v_{c, I V}(t)=\frac{\dot{\theta}_{I V}(t)}{K_{I V}}
$$

Sendo que em (3.38), $K_{I V}$ é o ganho do VCO do escravo do nível IV.

Para descrever a estabilidade do nó considerado, define-se o erro de fase:

$$
\varphi_{I I I, I V}(t) \stackrel{\Delta}{=} \Phi_{I I I}(t)-\Phi_{I V}(t), \quad \varphi_{I I I, I V}(t) \in[-\pi, \pi[
$$




$$
\begin{aligned}
& b_{I V} \cdot \dddot{\varphi}_{I I I, I V}(t)+c_{I I I} \cdot \ddot{\varphi}_{I I I, I V}(t)+\dot{\varphi}_{I I I, I V}(t)+a_{I V} \cdot \mu_{I V} \cdot \dot{\varphi}_{I I I, I V}(t) \cdot \cos \varphi_{I I I, I V}(t) \\
& +\mu_{I V} \cdot \operatorname{sen} \varphi_{I I I, I V}(t)=b_{I V} \cdot \dddot{\theta}_{I I I}(t)+c_{I V} \cdot \ddot{\theta}_{I I I}(t)+\dot{\theta}_{I I I}(t)
\end{aligned}
$$

Sendo que em (3.40), o seguinte parâmetro foi definido:

$$
\mu_{I V} \stackrel{\Delta}{=} \frac{K_{I V} \cdot K_{m, I V} \cdot V_{I I I} \cdot V_{I V}}{2}
$$

A seguir, será obtida a equação que descreve a dinâmica de um escravo do nível V.

\subsection{EQUAÇÃO DINÂMICA DE UM ESCRAVO DO NÍVEL V}

Em um escravo do nível $\mathrm{V}$, o sinal de entrada $v_{I V}(t)$ é proveniente do VCO de um escravo do nível IV. Este sinal é considerado periódico, sendo descrito pela equação (3.33).

$\mathrm{O}$ sinal que gerado pelo $\mathrm{VCO}$ do escravo $\mathrm{V}, v_{V}(t)$, também é considerado periódico. Ele será descrito pela seguinte equação:

$$
v_{V}(t)=V_{V} \cdot \cos \Phi_{V}(t)=V_{V} \cdot \cos \left[\omega_{0} \cdot t+\theta_{V}(t)\right]
$$

Observando-se as equações (3.33) e (3.42) é possível concluir que, a partir do escravo $\mathrm{V}$, não é necessário estudar os nós dos demais níveis. Por exemplo: um nó $\mathrm{V}$ pode ser equacionado a partir de um nó I, um nó VI, a partir de um nó II e assim sucessivamente.

A seguir, as equações que descrevem as dinâmicas dos nós dos níveis I, II, III e IV são generalizadas para se proceder ao estudo de um escravo qualquer da rede.

\subsection{EQUAÇÃO DINÂMICA DE UM NÓ ESCRAVO QUALQUER}

Para fins de generalização, as equações diferenciais que descrevem o sincronismo dos escravos I, II, III e IV estão reapresentadas a seguir:

$$
\begin{aligned}
& b_{I} \cdot \dddot{\varphi}_{M, I}(t)+c_{I} \cdot \ddot{\varphi}_{M, I}(t)+\dot{\varphi}_{M, I}(t)+a_{I} \cdot \mu_{I} \cdot \dot{\varphi}_{M, I}(t) \cdot \cos \varphi_{M, I}(t)+ \\
& +\mu_{I} \cdot \operatorname{sen} \varphi_{M, I}(t)=b_{I} \cdot \dddot{\theta}_{M}(t)+c_{I} \cdot \ddot{\theta}_{M}(t)+\dot{\theta}_{M}(t) \\
& b_{I I} \cdot \dddot{\varphi}_{I, I I}(t)+c_{I I} \cdot \ddot{\varphi}_{I, I I}(t)+\dot{\varphi}_{I, I I}(t)+a_{I I} \cdot \mu_{I I} \cdot \dot{\varphi}_{I, I I}(t) \cdot \cos \varphi_{I, I I}(t)+ \\
& +\mu_{I I} \cdot \operatorname{sen} \varphi_{I, I I}(t)=b_{I I} \cdot \dddot{\theta}_{I}(t)+c_{I I} \cdot \ddot{\theta}_{I}(t)+\dot{\theta}_{I}(t)
\end{aligned}
$$




$$
\begin{aligned}
& b_{I I I} \cdot \dddot{\varphi}_{I I, I I I}(t)+c_{I I I} \cdot \ddot{\varphi}_{I I, I I I}(t)+\dot{\varphi}_{I I, I I}(t)+a_{I I I} \cdot \mu_{I I I} \cdot \dot{\varphi}_{I I, I I I}(t) \cdot \cos \varphi_{I I, I I I}(t)+ \\
& +\mu_{I I I} \cdot \operatorname{sen} \varphi_{I I, I I I}(t)=b_{I I I} \cdot \dddot{\theta}_{I I}(t)+c_{I I I} \cdot \ddot{\theta}_{I I}(t)+\dot{\theta}_{I I}(t) \\
& b_{I V} \cdot \dddot{\varphi}_{I I I, I V}(t)+c_{I I I} \cdot \ddot{\varphi}_{I I I, I V}(t)+\dot{\varphi}_{I I I, I V}(t)+a_{I V} \cdot \mu_{I V} \cdot \dot{\varphi}_{I I I, I V}(t) \cdot \cos \varphi_{I I I, I V}(t) \\
& +\mu_{I V} \cdot \operatorname{sen} \varphi_{I I I, I V}(t)=b_{I V} \cdot \dddot{\theta}_{I I I}(t)+c_{I V} \cdot \ddot{\theta}_{I I I}(t)+\dot{\theta}_{I I I}(t)
\end{aligned}
$$

Observando as equações anteriores é possível fazer a seguinte generalização:

- Se $n=I$, então

$$
\begin{aligned}
& b_{I} \cdot \dddot{\varphi}_{M, I}(t)+c_{I} \cdot \ddot{\varphi}_{M, I}(t)+\dot{\varphi}_{M, I}(t)+a_{I} \cdot \mu_{I} \cdot \dot{\varphi}_{M, I}(t) \cdot \cos \varphi_{M, I}(t)+ \\
& +\mu_{I} \cdot \operatorname{sen} \varphi_{M, I}(t)=b_{I} \cdot \dddot{\theta}_{M}(t)+c_{I} \cdot \ddot{\theta}_{M}(t)+\dot{\theta}_{M}(t)
\end{aligned}
$$

- Se $n \neq I$, então

$$
\begin{aligned}
& b_{n} \cdot \dddot{\varphi}_{n-1, n}(t)+c_{n-1} \cdot \ddot{\varphi}_{n-1, n}(t)+\dot{\varphi}_{n-1, n}(t)+a_{n} \cdot \mu_{n} \cdot \dot{\varphi}_{n-1, n}(t) \cdot \cos \varphi_{n-1, n}(t) \\
& +\mu_{n} \cdot \operatorname{sen} \varphi_{n-1, n}(t)=b_{n} \cdot \dddot{\theta}_{n-1}(t)+c_{n} \cdot \ddot{\theta}_{n-1}(t)+\dot{\theta}_{n-1}(t)
\end{aligned}
$$

Exceto pelos índices, a diferença existente entre as equações (3.47) e (3.48) se encontra nos termos referentes à fase do sinal de entrada: em (3.47), os termos $\dot{\theta}_{M}(t), \ddot{\theta}_{M}(t)$ e $\dddot{\theta}_{M}(t)$ são conhecidos, em princípio. O mesmo não ocorre com $\dot{\theta}_{n-1}(t), \ddot{\theta}_{n-1}(t)$ e $\dddot{\theta}_{n-1}(t)$. Comparando as equações (2.27) e (3.47) de um modo qualitativo, nota-se que o estudo de um nó escravo I já foi realizado no capítulo 2. Pelo fato de se desconhecer os termos referentes ao sinal de entrada em (3.48), será apresentada uma solução analítica na seção seguinte, a fim de se proceder ao estudo qualitativo do sincronismo dos demais escravos da rede.

\subsection{SISTEMA DINÂMICO DE UM NÓ ESCRAVO QUE NÃO ESTEJA DIRETAMENTE CONECTADO AO MESTRE}

Um nó escravo $n, n \neq I$, ou seja, aquele que não esteja diretamente ligado ao nó mestre, pode ser esquematicamente representado como segue: 


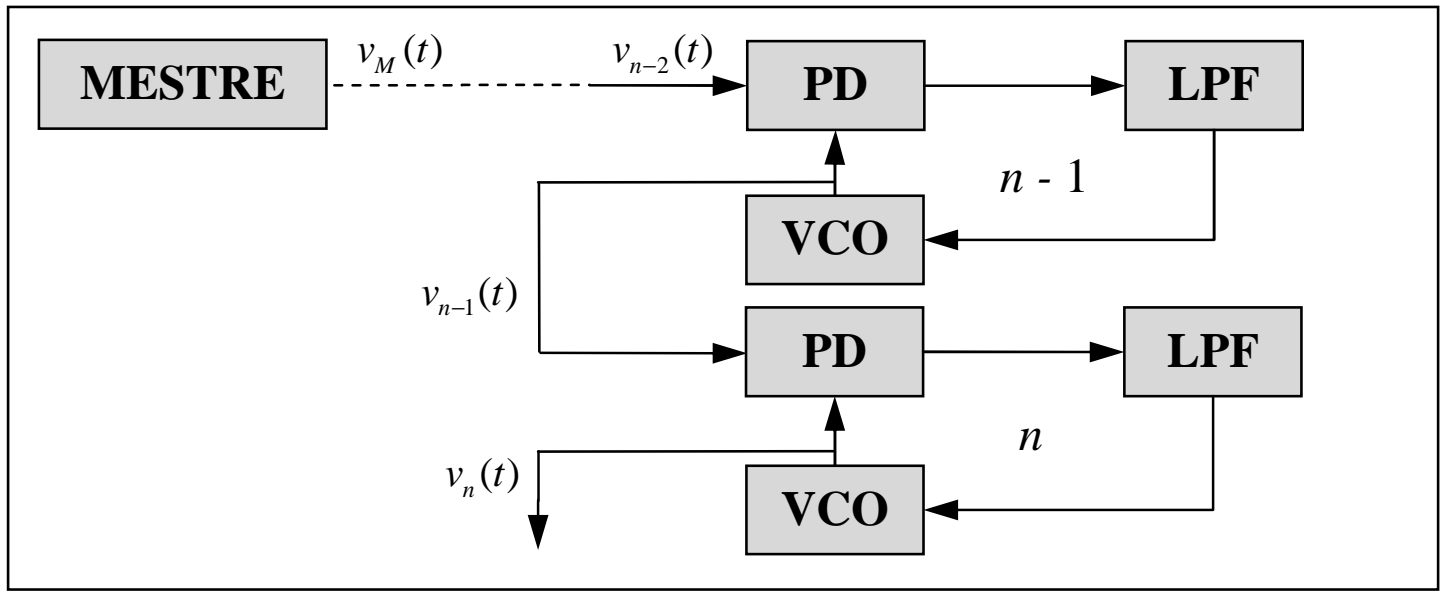

Fig.3.2: Diagrama em blocos de um escravo do nível $n, n \neq I$, de uma rede OWMS.

Para (3.47) é definido o seguinte erro de fase:

$$
\varphi_{n-1, n}(t)=\theta_{n-1}(t)-\theta_{n}(t)
$$

Então, define-se outro erro de fase, entre o escravo $n$ e o mestre:

$$
\varphi_{M, n}(t)=\theta_{M}(t)-\theta_{n}(t) \Rightarrow \theta_{n}(t)=\theta_{M}(t)-\varphi_{M, n}(t)
$$

Por outro lado, da mesma forma que em (3.47), é possível generalizar a equação diferencial (2.22) do capítulo II, como segue:

$$
\begin{aligned}
b_{n} \cdot \dddot{\theta}_{n}(t)+ & c_{n} \cdot \ddot{\theta}_{n}(t)+\dot{\theta}_{n}(t)=a_{n} \cdot \mu_{n} \cdot\left(\dot{\theta}_{n-1}(t)-\dot{\theta}_{n}(t)\right) \cdot \cos \left(\theta_{n-1}(t)-\theta_{n}(t)\right)+ \\
& +\mu_{n} \cdot \operatorname{sen}\left(\theta_{n-1}(t)-\theta_{n}(t)\right)
\end{aligned}
$$

Utilizando os erros de fase definidos em (3.49) e (3.50) na equação (3.51), tem-se:

$$
\begin{aligned}
& b_{n} \cdot\left[\dddot{\theta}_{M}(t)-\dddot{\varphi}_{M, n}(t)\right]+c_{n} \cdot\left[\ddot{\theta}_{M}(t)-\ddot{\varphi}_{M, n}(t)\right]+\left[\dot{\theta}_{M}(t)-\dot{\varphi}_{M, n}(t)\right]= \\
& =a_{n} \cdot \mu_{n} \cdot \dot{\varphi}_{n-1, n}(t) \cdot \cos \varphi_{n-1, n}(t)+\mu_{n} \cdot \operatorname{sen} \varphi_{n-1, n}(t)
\end{aligned}
$$

Pelo desenvolvimento das multiplicações entre colchetes, tem-se:

$$
\begin{aligned}
& b_{n} \cdot \dddot{\theta}_{M}(t)+c_{n} \cdot \ddot{\theta}_{M}(t)+\dot{\theta}_{M}(t)= \\
& =b_{n} \cdot \dddot{\varphi}_{M, n}(t)+c_{n} \cdot \ddot{\varphi}_{M, n}(t)+\dot{\varphi}_{M, n}(t)+a_{n} \cdot \mu_{n} \cdot \dot{\varphi}_{n-1, n}(t) \cdot \cos \varphi_{n-1, n}(t)+ \\
& \quad+\mu_{n} \cdot \operatorname{sen} \varphi_{n-1, n}(t)
\end{aligned}
$$

E assim: 


$$
\begin{aligned}
& b_{n} \cdot \dddot{\varphi}_{M, n}(t)+c_{n} \cdot \ddot{\varphi}_{M, n}(t)+\dot{\varphi}_{M, n}(t)+a_{n} \cdot \mu_{n} \cdot \dot{\varphi}_{n-1, n}(t) \cdot \cos \varphi_{n-1, n}(t)+ \\
& +\mu_{n} \cdot \operatorname{sen} \varphi_{n-1, n}(t)=b_{n} \cdot \dddot{\theta}_{M}(t)+c_{n} \cdot \ddot{\theta}_{M}(t)+\dot{\theta}_{M}(t)
\end{aligned}
$$

Na equação (3.54) há duas incógnitas: o erro de fase $\varphi_{n-1, n}(t)$ entre o escravo $n$ e o escravo $n$ - 1 e o erro de fase $\varphi_{M, n}(t)$ entre o escravo $n$ e o mestre. Portanto, é necessária outra equação diferencial. Define-se então, um terceiro erro de fase $\varphi_{M, n}(t)$ entre o escravo $n$ - 1 e o mestre:

$$
\varphi_{M, n-1}(t)=\theta_{M}(t)-\theta_{n-1}(t) \Rightarrow \theta_{n-1}(t)=\theta_{M}(t)-\varphi_{M, n-1}(t)
$$

Utilizando (3.55) na equação (3.48), tem-se:

$$
\begin{aligned}
& b_{n} \cdot \dddot{\varphi}_{n-1, n}(t)+c_{n} \cdot \ddot{\varphi}_{n-1, n}(t)+\dot{\varphi}_{n-1, n}(t)+a_{n} \cdot \mu_{n} \cdot \dot{\varphi}_{n-1, n}(t) \cdot \cos \varphi_{n-1, n}(t) \\
& +\mu_{n} \cdot \operatorname{sen} \varphi_{n-1, n}(t)=b_{n} \cdot\left[\dddot{\theta}_{M}(t)-\dddot{\varphi}_{M, n-1}(t)\right]+c_{n} \cdot\left[\ddot{\theta}_{M}(t)-\ddot{\varphi}_{M, n-1}(t)\right]+ \\
& +\left[\dot{\theta}_{M}(t)-\dot{\varphi}_{M, n-1}(t)\right]
\end{aligned}
$$

E assim:

$$
\begin{aligned}
& b_{n} \cdot \dddot{\varphi}_{n-1, n}(t)+c_{n} \cdot \ddot{\varphi}_{n-1, n}(t)+\dot{\varphi}_{n-1, n}(t)+ \\
& +a_{n} \cdot \mu_{n} \cdot \dot{\varphi}_{n-1, n}(t) \cdot \cos \varphi_{n-1, n}(t)+\mu_{n} \cdot \operatorname{sen} \varphi_{n-1, n}(t)= \\
& =b_{n} \cdot \dddot{\theta}_{M}(t)+c_{n} \cdot \ddot{\theta}_{M}(t)+\dot{\theta}_{M}(t)-b_{n} \cdot \dddot{\varphi}_{M, n-1}(t)-c_{n} \cdot \ddot{\varphi}_{M, n-1}(t)-\dot{\varphi}_{M, n-1}(t)
\end{aligned}
$$

Com as equações (3.54) e (3.57), pode-se escrever o seguinte sistema dinâmico:

$$
\left\{\begin{array}{l}
b_{n} \cdot \dddot{\varphi}_{M, n}(t)+c_{n} \cdot \ddot{\varphi}_{M, n}(t)+\dot{\varphi}_{M, n}(t)+a_{n} \cdot \mu_{n} \cdot \dot{\varphi}_{n-1, n}(t) \cdot \cos \varphi_{n-1, n}(t)+ \\
+\mu_{n} \cdot \operatorname{sen} \varphi_{n-1, n}(t)=b_{n} \cdot \dddot{\theta}_{M}(t)+c_{n} \cdot \ddot{\theta}_{M}(t)+\dot{\theta}_{M}(t) \\
b_{n} \cdot \dddot{\varphi}_{n-1, n}(t)+c_{n} \cdot \ddot{\varphi}_{n-1, n}(t)+\dot{\varphi}_{n-1, n}(t)+ \\
+a_{n} \cdot \mu_{n} \cdot \dot{\varphi}_{n-1, n}(t) \cdot \cos \varphi_{n-1, n}(t)+\mu_{n} \cdot \operatorname{sen} \varphi_{n-1, n}(t)= \\
=b_{n} \cdot \dddot{\theta}_{M}(t)+c_{n} \cdot \ddot{\theta}_{M}(t)+\dot{\theta}_{M}(t)-b_{n} \cdot \dddot{\varphi}_{M, n-1}(t)-c_{n} \cdot \ddot{\varphi}_{M, n-1}(t)-\dot{\varphi}_{M, n-1}(t)
\end{array}\right.
$$

Caso se admita, por simplificação, que para $t$ suficientemente grande o nó $n$ - 1 já sincronizou com o mestre, pode-se proceder à seguinte simplificação:

$$
\dot{\varphi}_{M, n-1}(t)=0, \ddot{\varphi}_{M, n-1}(t)=0, \dddot{\varphi}_{M, n-1}(t)=0 \text { e } \dddot{\varphi}_{M, n-1}(t)=0
$$

Dessa forma se obtém: 


$$
\left\{\begin{array}{l}
b_{n} \cdot \dddot{\varphi}_{M, n}(t)+c_{n} \cdot \ddot{\varphi}_{M, n}(t)+\dot{\varphi}_{M, n}(t)+a_{n} \cdot \mu_{n} \cdot \dot{\varphi}_{n-1, n}(t) \cdot \cos \varphi_{n-1, n}(t)+ \\
+\mu_{n} \cdot \operatorname{sen} \varphi_{n-1, n}(t)=b_{n} \cdot \dddot{\theta}_{M}(t)+c_{n} \cdot \ddot{\theta}_{M}(t)+\dot{\theta}_{M}(t) \\
b_{n} \cdot \dddot{\varphi}_{n-1, n}(t)+c_{n} \cdot \ddot{\varphi}_{n-1, n}(t)+\dot{\varphi}_{n-1, n}(t)+a_{n} \cdot \mu_{n} \cdot \dot{\varphi}_{n-1, n}(t) \cdot \cos \varphi_{n-1, n}(t) \\
+\mu_{n} \cdot \operatorname{sen} \varphi_{n-1, n}(t)=b_{n} \cdot \dddot{\theta}_{M}(t)+c_{n} \cdot \ddot{\theta}_{M}(t)+\dot{\theta}_{M}(t)
\end{array}\right.
$$

Observando o sistema dinâmico (3.60) é possível concluir que para descrever o sincronismo entre o nó $n$ e o nó $n$ - 1 é suficiente que se estude somente uma de suas equações. Assim, ao se considerar que o nó $n-1$ está sincronizado com o mestre, tanto faz estudar o sincronismo entre o nó $n$ e o mestre, como entre os nós $n$ e $n-1$. Como esse último estudo já foi realizado no capítulo II, surge uma questão relevante: como estudar qualitativamente um nó escravo que não esteja diretamente conectado ao mestre? Caso se conheça a expressão do sinal de entrada, o estudo é imediato. No caso contrário, basta proceder às manipulações algébricas desta seção. Entretanto, nessa opção há duas desvantagens: a primeira é a criação de mais uma equação diferencial, que acaba por compor um sistema de duas equações diferencias de terceira ordem, não-homogêneas e não-lineares. A segunda é a criação de termos relativos ao sincronismo entre o nó antecedente e o mestre. Então, caso se adote a hipótese que o nó antecedente já sincronizou com o mestre, não é mais necessário estudar as duas equações do sistema, mas somente a segunda, que descreve o sincronismo entre o nó escravo em questão e o nó escravo que o antecede. Uma alternativa a essa simplificação é equacionar todos os nós que perfazem o caminho entre o mestre e o escravo que se deseje estudar. Quanto maior o número de nós neste caminho, maior o número de equações, portanto. Dessa forma, na próxima seção será realizado o equacionamento de uma rede com um mestre, um escravo I e outro II.

\subsection{SISTEMA DINÂMICO PARA UM MESTRE, UM NÓ ESCRAVO I E UM NÓ ESCRAVO II}

Aplicando-se a equação (2.22) do capítulo 2 para o estudo do nó II, segue que: 


$$
\begin{aligned}
b_{I I} \cdot \dddot{\theta}_{I I}(t) & +c_{I I} \cdot \ddot{\theta}_{I I}(t)+\dot{\theta}_{I I}(t)=a_{I I} \cdot \mu_{I I} \cdot\left(\dot{\theta}_{I}(t)-\dot{\theta}_{I I}(t)\right) \cdot \cos \left(\theta_{I}(t)-\theta_{I I}(t)\right)+ \\
& +\mu_{I I} \cdot \operatorname{sen}\left(\theta_{I}(t)-\theta_{I I}(t)\right)
\end{aligned}
$$

Por outro lado é possível definir os seguintes erros de fase:

$$
\begin{aligned}
& \varphi_{M, I}(t)=\theta_{M}(t)-\theta_{I}(t) \\
& \varphi_{M, I I}(t)=\theta_{M}(t)-\theta_{I I}(t) \\
& \varphi_{I, I I}(t)=\theta_{I}(t)-\theta_{I I}(t)
\end{aligned}
$$

Utilizando as equações (3.63) e (3.64) na equação (3.61), tem-se:

$$
\begin{aligned}
& b_{I I} \cdot\left[\dddot{\theta}_{M}(t)-\dddot{\varphi}_{M, I I}(t)\right]+c_{I I} \cdot\left[\ddot{\theta}_{M}(t)-\ddot{\varphi}_{M, I I}(t)\right]+\left[\dot{\theta}_{M}(t)-\dot{\varphi}_{M, I I}(t)\right]= \\
& =a_{I I} \cdot \mu_{I I} \cdot\left(\dot{\varphi}_{I, I I}(t)\right) \cdot \cos \left(\varphi_{I, I I}(t)\right)+\mu_{I I} \cdot \operatorname{sen}\left(\varphi_{I, I I}(t)\right)
\end{aligned}
$$

Por outro lado, de (3.62) e (3.64) vem que:

$\left\{\begin{array}{l}\varphi_{M, I}(t)=\theta_{M}(t)-\theta_{I}(t) \\ \varphi_{I, I I}(t)=\theta_{I}(t)-\theta_{I I}(t)\end{array} \Rightarrow \varphi_{M, I}(t)+\varphi_{I, I I}(t)=\theta_{M}(t)-\theta_{I I}(t)\right.$

Ainda, pela definição (3.63) em (3.66), tem-se que:

$$
\varphi_{M, I}(t)+\varphi_{I, I I}(t)=\varphi_{M, I I}(t) \Rightarrow \varphi_{I, I I}(t)=\varphi_{M, I I}(t)-\varphi_{M, I}(t)
$$

Agora, tomando o resultado obtido em (3.67) na equação (3.65), tem-se:

$$
\begin{aligned}
& b_{I I} \cdot\left[\dddot{\theta}_{M}(t)-\dddot{\varphi}_{M, I I}(t)\right]+c_{I I} \cdot\left[\ddot{\theta}_{M}(t)-\ddot{\varphi}_{M, I I}(t)\right]+\left[\dot{\theta}_{M}(t)-\dot{\varphi}_{M, I I}(t)\right]= \\
& =a_{I I} \cdot \mu_{I I} \cdot\left(\dot{\varphi}_{M, I I}(t)-\dot{\varphi}_{M, I}(t)\right) \cdot \cos \left(\varphi_{M, I I}(t)-\varphi_{M, I}(t)\right)+ \\
& \quad+\mu_{I I} \cdot \operatorname{sen}\left(\varphi_{M, I I}(t)-\varphi_{M, I}(t)\right)
\end{aligned}
$$

Assim, desenvolvendo (3.68), tem-se:

$$
\begin{aligned}
& b_{I I} \cdot \dddot{\varphi}_{M, I I}(t)+c_{I I} \cdot \ddot{\varphi}_{M, I I}(t)+\dot{\varphi}_{M, I I}(t)+ \\
& +a_{I I} \cdot \mu_{I I} \cdot\left(\dot{\varphi}_{M, I I}(t)-\dot{\varphi}_{M, I}(t)\right) \cdot \cos \left(\varphi_{M, I I}(t)-\varphi_{M, I}(t)\right)+ \\
& \quad+\mu_{I I} \cdot \operatorname{sen}\left(\varphi_{M, I I}(t)-\varphi_{M, I}(t)\right)= \\
& =b_{I I} \cdot \dddot{\theta}_{M}(t)+c_{I I} \cdot \ddot{\theta}_{M}(t)+\dot{\theta}_{M}(t)
\end{aligned}
$$


Na equação (3.69), há duas incógnitas: o erro de fase $\varphi_{M, I}(t)$ entre o escravo I e o mestre e o erro de fase $\varphi_{M, I I}(t)$ entre o escravo II e o mestre. Portanto, é necessária outra equação diferencial. Será utilizada a equação (3.42), que já foi obtida. Então, o sistema dinâmico que descreve o escravo II é:

$$
\left\{\begin{array}{l}
b_{I} \cdot \dddot{\varphi}_{M, I}(t)+c_{I} \cdot \ddot{\varphi}_{M, I}(t)+\dot{\varphi}_{M, I}(t)+a_{I} \cdot \mu_{I} \cdot \dot{\varphi}_{M, I}(t) \cdot \cos \varphi_{M, I}(t)+ \\
+\mu_{I} \cdot \operatorname{sen} \varphi_{M, I}(t)=b_{I} \cdot \dddot{\theta}_{M}(t)+c_{I} \cdot \ddot{\theta}_{M}(t)+\dot{\theta}_{M}(t) \\
b_{I I} \dddot{\varphi}_{M, I I}(t)+c_{I I} \cdot \ddot{\varphi}_{M, I I}(t)+\dot{\varphi}_{M, I I}(t)+ \\
+a_{I I} \cdot \mu_{I I} \cdot\left(\dot{\varphi}_{M, I I}(t)-\dot{\varphi}_{M, I}(t)\right) \cdot \cos \left(\varphi_{M, I I}(t)-\varphi_{M, I}(t)\right)+ \\
\quad+\mu_{I I} \cdot \operatorname{sen}\left(\varphi_{M, I I}(t)-\varphi_{M, I}(t)\right)= \\
=b_{I I} \cdot \dddot{\theta}_{M}(t)+c_{I I} \cdot \ddot{\theta}_{M}(t)+\dot{\theta}_{M}(t)
\end{array}\right.
$$

Nos próximos capítulos, será realizado estudo de um escravo tipo II, descrito por (3.69), frente a variações na fase do nó mestre. No capítulo IV, a variação é do tipo degrau de fase e no capítulo $\mathrm{V}$, do tipo rampa. 


\section{CAPÍTULO IV}

\section{ESTUDO DA ESTABILIDADE DE UM NÓ ESCRAVO TIPO II FRENTE A UMA VARIAÇÃO DO TIPO DEGRAU DE FASE NO MESTRE.}

No capítulo III foi realizado um estudo geral dos nós escravos de uma rede OWMS Mista Estrela-Cadeia Simples. Para nós que não estão diretamente conectados ao mestre se adotou a hipótese simplificadora de que o nó antecedente já estaria sincronizado com o mestre, depois de um intervalo de tempo suficientemente longo. Neste capítulo, apresenta-se o estudo nó escravo tipo II, sem adotar essa simplificação. A análise da estabilidade será realizada frente a uma variação do tipo degrau de fase no mestre.

\subsection{OBTENÇÃO DOS PONTOS DE EQUILÍBRIO}

Conforme apresentado no capítulo 3 em (3.69), o sistema dinâmico que descreve um escravo II é da forma:

$$
\left\{\begin{array}{l}
b_{I} \cdot \dddot{\varphi}_{M, I}(t)+c_{I} \cdot \ddot{\varphi}_{M, I}(t)+\dot{\varphi}_{M, I}(t)+a_{I} \cdot \mu_{I} \cdot \dot{\varphi}_{M, I}(t) \cdot \cos \varphi_{M, I}(t)+ \\
+\mu_{I} \cdot \operatorname{sen} \varphi_{M, I}(t)=b_{I} \cdot \dddot{\theta}_{M}(t)+c_{I} \cdot \ddot{\theta}_{M}(t)+\dot{\theta}_{M}(t) \\
b_{I I} \cdot \dddot{\varphi}_{M, I I}(t)+c_{I I} \cdot \ddot{\varphi}_{M, I I}(t)+\dot{\varphi}_{M, I I}(t)+ \\
+a_{I I} \cdot \mu_{I I} \cdot\left(\dot{\varphi}_{M, I I}(t)-\dot{\varphi}_{M, I}(t)\right) \cdot \cos \left(\varphi_{M, I I}(t)-\varphi_{M, I}(t)\right)+ \\
\quad+\mu_{I I} \cdot \operatorname{sen}\left(\varphi_{M, I I}(t)-\varphi_{M, I}(t)\right)= \\
=b_{I I} \cdot \dddot{\theta}_{M}(t)+c_{I I} \cdot \ddot{\theta}_{M}(t)+\dot{\theta}_{M}(t)
\end{array}\right.
$$

Para variações $\theta_{M}(t)$ do tipo degrau de fase, pode-se escrever que:

$$
\dot{\theta}_{M}(t)=0 \Rightarrow \ddot{\theta}_{M}(t)=0 \Rightarrow \dddot{\theta}_{M}(t)=0
$$

Substituindo as entradas descritas em (4.2) no sistema dinâmico (4.1), tem-se: 


$$
\left\{\begin{aligned}
& b_{I} \cdot \dddot{\varphi}_{M, I}(t)+c_{I} \cdot \ddot{\varphi}_{M, I}(t)+\dot{\varphi}_{M, I}(t)+a_{I} \cdot \mu_{I} \cdot \dot{\varphi}_{M, I}(t) \cdot \cos \varphi_{M, I}(t)+ \\
&+\mu_{I} \cdot \operatorname{sen} \varphi_{M, I}(t)=0 \\
& b_{I I} \cdot \dddot{\varphi}_{M, I I}(t)+c_{I I} \cdot \ddot{\varphi}_{M, I I}(t)+\dot{\varphi}_{M, I I}(t)+ \\
&+a_{I I} \cdot \mu_{I I} \cdot\left(\dot{\varphi}_{M, I I}(t)-\dot{\varphi}_{M, I}(t)\right) \cdot \cos \left(\varphi_{M, I I}(t)-\varphi_{M, I}(t)\right)+ \\
&+\mu_{I I} \cdot \operatorname{sen}\left(\varphi_{M, I I}(t)-\varphi_{M, I}(t)\right)=0
\end{aligned}\right.
$$

Para a obtenção do sistema de equações no espaço de estados de (4.3), definese as seguintes variáveis de estado:

$$
\left\{\begin{array}{l}
x_{1}=\varphi_{M, I} \\
x_{2}=\dot{\varphi}_{M, I} \\
x_{3}=\ddot{\varphi}_{M, I} \\
x_{4}=\varphi_{M, I I} \\
x_{5}=\dot{\varphi}_{M, I I} \\
x_{6}=\ddot{\varphi}_{M, I I}
\end{array}\right.
$$

Assim, para $b_{n} \neq 0$, tem-se o seguinte sistema de equações no espaço de estados:

$$
\left\{\begin{array}{l}
\dot{x}_{1}=x_{2} \\
\dot{x}_{2}=x_{3} \\
\dot{x}_{3}=-\frac{c_{I}}{b_{I}} x_{3}-\frac{1}{b_{I}} x_{2}-\frac{\mu_{I} \cdot a_{I}}{b_{I}} x_{2} \cos x_{1}-\frac{\mu_{I}}{b_{I}} \operatorname{sen} x_{1} \\
\dot{x}_{4}=x_{5} \\
\dot{x}_{5}=x_{6} \\
\dot{x}_{6}=-\frac{c_{I I}}{b_{I I}} x_{6}-\frac{1}{b_{I I}} x_{5}-\frac{\mu_{I I} \cdot a_{I I}}{b_{I I}}\left(x_{5}-x_{2}\right) \cos \left(x_{4}-x_{1}\right)-\frac{\mu_{I I}}{b_{I I}} \operatorname{sen}\left(x_{4}-x_{1}\right)
\end{array}\right.
$$

No sistema (4.5), os pontos de equilíbrio podem ser obtidos como segue: 


$$
\begin{aligned}
& \left\{\begin{array}{l}
\dot{x}_{1}=0 \Rightarrow x_{2}^{*}=0 \\
\dot{x}_{2}=0 \Rightarrow x_{3}^{*}=0
\end{array}\right. \\
& \dot{x}_{3}=0 \Rightarrow-\frac{\mu_{I}}{b_{I}} \operatorname{sen} x_{1}^{*}=0 \Rightarrow\left\{\begin{array}{l}
x_{1}^{*}=0 \\
\text { ou } \\
x_{1}^{*}=-\pi
\end{array}\right. \\
& \dot{x}_{4}=0 \Rightarrow x_{5}^{*}=0 \\
& \dot{x}_{5}=0 \Rightarrow x_{6}^{*}=0 \\
& \dot{x}_{6}=0 \Rightarrow-\frac{\mu_{I I}}{b_{I I}} \operatorname{sen}\left(x_{4}^{*}-x_{1}^{*}\right)=0 \Rightarrow\left\{\begin{array}{l}
x_{1}^{*}=0 \Rightarrow\left\{\begin{array}{l}
x_{4}^{*}=0 \\
\text { ou } \\
x_{4}^{*}=-\pi
\end{array}\right. \\
\text { ou } \\
x_{1}^{*}=-\pi \Rightarrow\left\{\begin{array}{l}
x_{4}^{*}=-\pi \\
\text { ou } \\
x_{4}^{*}=0
\end{array}\right.
\end{array}\right.
\end{aligned}
$$

Portanto, os pontos de equilíbrio de (4.6) são:

$$
\begin{aligned}
& P_{1}=(0,0,0,0,0,0), P_{2}=(-\pi, 0,0,0,0,0), P_{3}=(-\pi, 0,0,-\pi, 0,0) \mathrm{e} \\
& P_{2}=(0,0,0,-\pi, 0,0)
\end{aligned}
$$

A estabilidade destes pontos será discutida nas próximas subseções.

\subsubsection{ANÁLISE LINEAR DA ESTABILIDADE DOS PONTOS DE} EQUILÍBRIO $P_{1}=(0,0,0,0,0,0), P_{2}=(-\pi, 0,0,0,0,0), P_{3}=(-\pi, 0,0,-\pi, 0,0) \quad \mathbf{E}$ $P_{2}=(0,0,0,-\pi, 0,0)$

Para iniciar a análise linear da estabilidade dos pontos de equilíbrio, simplifica-se o sistema (4.5) do mesmo modo como foi realizado para alguns casos do capítulo II. Entretanto, a proposta agora é transformar não um, mas dois parâmetros, $\mu_{I}$ e $\mu_{I I}$, em variáveis de estado através da inclusão de duas novas equações diferenciais sem dinâmica, $\dot{\mu}_{I}=0$ e $\dot{\mu}_{I I}=0$. Desse modo, o sistema (4.5) fica reescrito como segue: 


$$
\begin{aligned}
& \left\{\begin{array}{l}
\dot{x}_{1}=x_{2} \\
\dot{x}_{2}=x_{3} \\
\dot{x}_{3}=-\frac{c_{I}}{b_{I}} x_{3}-\frac{1}{b_{I}} x_{2}-\frac{\mu_{I} \cdot a_{I}}{b_{I}} x_{2} \cos x_{1}-\frac{\mu_{I}}{b_{I}} \operatorname{sen} x_{1} \\
\dot{x}_{4}=x_{5} \\
\dot{x}_{5}=x_{6} \\
\dot{x}_{6}=-\frac{c_{I I}}{b_{I I}} x_{6}-\frac{1}{b_{I I}} x_{5}-\frac{\mu_{I I} \cdot a_{I I}}{b_{I I}}\left(x_{5}-x_{2}\right) \cos \left(x_{4}-x_{1}\right)-\frac{\mu_{I I}}{b_{I I}} \operatorname{sen}\left(x_{4}-x_{1}\right)
\end{array}\right. \\
& \dot{\mu}_{I}=0 \text { e } \dot{\mu}_{I I}=0
\end{aligned}
$$

Então, a matriz Jacobiana de (4.8) calculada em $P_{1}, P_{2}, P_{3}$ ou $P_{4}$ é:

$$
J_{P_{1,2,3,4}}=\left[\begin{array}{cccccc}
0 & 1 & 0 & 0 & 0 & 0 \\
0 & 0 & 1 & 0 & 0 & 0 \\
0 & -\frac{1}{b_{I}} & -\frac{c_{I}}{b_{I}} & 0 & 0 & 0 \\
0 & 0 & 0 & 0 & 1 & 0 \\
0 & 0 & 0 & 0 & 0 & 1 \\
0 & 0 & 0 & 0 & -\frac{1}{b_{I I}} & -\frac{c_{I I}}{b_{I I}}
\end{array}\right]
$$

Por simplicidade, as linhas e as colunas de (4.9) correspondentes às novas variáveis $\mu_{I}$ e $\mu_{I I}$ estão sendo omitidas, uma vez que sua introdução não alterou a dinâmica do sistema.

Assim, os autovalores de (4.9) são:

- $\lambda_{1}=0$

- $\lambda_{2}=0$

- $\lambda_{3}=-\frac{\left(c_{I}+\sqrt{c_{I}^{2}-4 b_{I}}\right)}{2 b_{I}}$

- $\lambda_{4}=\frac{\left(\sqrt{c_{I}^{2}-4 b_{I}}-c_{I}\right)}{2 b_{I}}$

- $\lambda_{5}=-\frac{\left(c_{I I}+\sqrt{c_{I I}^{2}-4 b_{I I}}\right)}{2 b_{I I}}$ 
- $\lambda_{6}=\frac{\left(\sqrt{c_{I I}^{2}-4 b_{I I}}-c_{I I}\right)}{2 b_{I I}}$

Através da análise dos autovalores de (4.10), pode-se verificar que:

- Os autovalores $\lambda_{1}$ e $\lambda_{2}$ são nulos, independentemente dos valores ou relações que possam assumir os parâmetros envolvidos.

- Os autovalores $\lambda_{3}$ e $\lambda_{4}$ são ou reais com parte real negativa, para $c_{I}^{2} \geq 4 b_{I}$, ou pares de complexos conjugados com parte real negativa, para $c_{I}^{2}<4 b_{I}$.

- Os autovalores $\lambda_{5}$ e $\lambda_{6}$ são ou reais com parte real negativa, para $c_{I I}^{2} \geq 4 b_{I I}$, ou pares de complexos conjugados com parte real negativa, para $c_{I I}^{2}<4 b_{I I}$.

Portanto, graças à existência de dois autovalores nulos, não é possível obter conclusões sobre a estabilidade de $P_{1}, P_{2}, P_{3}$ ou $P_{4}$ através da análise linear. Para investigar o comportamento do sistema no subespaço central, será aplicado o Teorema da Variedade Central (TVC).

\subsubsection{ANÁLISE DA ESTABILIDADE DE $P_{1}=(0,0,0,0,0,0)$ VIA TVC}

Para a aplicação do TVC, faz-se necessária a expansão em série de Taylor das funções seno e co-seno em torno de $x_{1}^{*}=0$ e $x_{4}^{*}=0$ :

$$
\operatorname{sen} x_{1} \approx x_{1}+\ldots, \cos x_{1} \approx 1+\ldots, \operatorname{sen} x_{4} \approx x_{4}+\ldots \text { e } \cos x_{4} \approx 1+\ldots
$$

E, portanto, a partir de (4.11), tem-se:

- $\cos \left(x_{4}-x_{1}\right)=\cos x_{4} \cos x_{1}+\operatorname{sen} x_{4} \operatorname{sen} x_{1}=1+x_{4} \cdot x_{1}$

- $\operatorname{sen}\left(x_{4}-x_{1}\right)=\operatorname{sen} x_{4} \cos x_{1}-\operatorname{sen} x_{1} \cos x_{4}=x_{4}-x_{1}$

Utilizando as expressões de (4.12), reescreve-se o sistema (4.8): 


$$
\begin{aligned}
& \left\{\begin{array}{l}
\dot{x}_{1}=x_{2} \\
\dot{x}_{2}=x_{3} \\
\dot{x}_{3}=-\frac{c_{I}}{b_{I}} x_{3}-\frac{1}{b_{I}} x_{2}-\frac{\mu_{I} \cdot a_{I}}{b_{I}} x_{2}-\frac{\mu_{I}}{b_{I}} x_{1} \\
\dot{x}_{4}=x_{5} \\
\dot{x}_{5}=x_{6} \\
\dot{x}_{6}=-\frac{c_{I I}}{b_{I I}} x_{6}-\frac{1}{b_{I I}} x_{5}-\frac{\mu_{I I} \cdot a_{I I}}{b_{I I}}\left(x_{5}-x_{2}\right)\left[1+x_{4} \cdot x_{1}\right]-\frac{\mu_{I I}}{b_{I I}}\left[x_{4}-x_{1}\right]
\end{array}\right. \\
& \dot{\mu}_{I}=0 \text { e } \dot{\mu}_{I I}=0
\end{aligned}
$$

O sistema (4.13) pode ser representado matricialmente como $\dot{X}=J_{P_{1,2}} \cdot X+F$, $\dot{\mu}_{I}=0$ e $\dot{\mu}_{I I}=0$, ou seja:

$$
\left[\begin{array}{l}
\dot{x}_{1} \\
\dot{x}_{2} \\
\dot{x}_{3} \\
\dot{x}_{4} \\
\dot{x}_{5} \\
\dot{x}_{6}
\end{array}\right]=\left[\begin{array}{cccccc}
0 & 1 & 0 & 0 & 0 & 0 \\
0 & 0 & 1 & 0 & 0 & 0 \\
0 & -\frac{1}{b_{I}} & -\frac{c_{I}}{b_{I}} & 0 & 0 & 0 \\
0 & 0 & 0 & 0 & 1 & 0 \\
0 & 0 & 0 & 0 & 0 & 1 \\
0 & 0 & 0 & 0 & -\frac{1}{b_{I I}} & -\frac{c_{I I}}{b_{I I}}
\end{array}\right] \cdot\left[\begin{array}{c}
x_{1} \\
x_{2} \\
x_{3} \\
x_{4} \\
x_{5} \\
x_{6}
\end{array}\right]+
$$

$$
+\left[\begin{array}{c}
0 \\
0 \\
-\frac{\mu_{I} \cdot a_{I}}{b_{I}} x_{2}-\frac{\mu_{I}}{b_{I}} x_{1} \\
0 \\
0 \\
-\frac{\mu_{I I} \cdot a_{I I}}{b_{I I}}\left(x_{5}-x_{2}\right)\left(1+x_{4} \cdot x_{1}\right)-\frac{\mu_{I I}}{b_{I I}}\left(x_{4}-x_{1}\right)
\end{array}\right],
$$

Conforme requerido pelo TVC, o sistema (4.12) precisa ser reescrito na forma canônica de Jordan. Para isso, define-se um novo conjunto de variáveis de estado $V=\left(v_{1}, v_{2}, v_{3}, v_{4}, v_{5}, v_{6}\right)^{T}$ de modo que $X=M \cdot V$, sendo $M$ uma base composta pelos 
autovetores de $J_{P_{1,2,3,4}}$ de (5.9). Dessa forma, é possível dizer que

$$
\begin{gathered}
V=\left[M^{-1} \cdot J_{P_{1,2}} \cdot M\right] \cdot V+M^{-1} \cdot F \text {, ou seja: } \\
{\left[\begin{array}{c}
\dot{v}_{1} \\
\dot{v}_{2} \\
\dot{v}_{3} \\
\dot{v}_{4} \\
\dot{v}_{5} \\
\dot{v}_{6}
\end{array}\right]=\left[\begin{array}{cccccc}
\lambda_{1} & 0 & 0 & 0 & 0 & 0 \\
0 & \lambda_{2} & 0 & 0 & 0 & 0 \\
0 & 0 & \lambda_{3} & 0 & 0 & 0 \\
0 & 0 & 0 & \lambda_{4} & 0 & 0 \\
0 & 0 & 0 & 0 & \lambda_{5} & 0 \\
0 & 0 & 0 & 0 & 0 & \lambda_{6}
\end{array}\right] \cdot\left[\begin{array}{l}
v_{1} \\
v_{2} \\
v_{3} \\
v_{4} \\
v_{5} \\
v_{6}
\end{array}\right]+\left[\begin{array}{l}
f_{1} \\
f_{2} \\
f_{3} \\
f_{4} \\
f_{5} \\
f_{6}
\end{array}\right]} \\
\dot{\mu}_{I}=0 \text { e } \dot{\mu}_{I I}=0
\end{gathered}
$$

Em (4.13), tem-se:

- $\lambda_{1}=0$

- $\lambda_{2}=0$

- $\lambda_{3}=-\frac{\left(c_{I}+\sqrt{c_{I}^{2}-4 b_{I}}\right)}{2 b_{I}}$

- $\lambda_{4}=\frac{\left(\sqrt{c_{I}^{2}-4 b_{I}}-c_{I}\right)}{2 b_{I}}$

- $\lambda_{5}=-\frac{\left(c_{I I}+\sqrt{c_{I I}^{2}-4 b_{I I}}\right)}{2 b_{I I}}$

- $\lambda_{6}=\frac{\left(\sqrt{c_{I I}^{2}-4 b_{I I}}-c_{I I}\right)}{2 b_{I I}}$

- $f_{1}=\frac{1}{2} \mu_{I I}\left\{2\left(-v_{1}+v_{2}\right)-\left(2 b_{I}+\sqrt{1-4 b_{I}}-1\right) v_{3}+\left(-2 b_{I}+\sqrt{1-4 b_{I}}+1\right) v_{4}+\right.$

$+\left[2 b_{I I}+c_{I I}\left(\sqrt{c_{I I}^{2}-4 b_{I I}}-c_{I I}\right) v_{5}\right]+\left[c_{I I}\left(c_{I I}+\sqrt{c_{I I}^{2}-4 b_{I I}}-2 b_{I I}\right) v_{6}\right]-$

$-a_{I I}\left[-\left(\sqrt{1-4 b_{I}}-1\right) v_{3}+\left(\sqrt{1-4 b_{I}}+1\right) v_{4}-\right.$

$\left.-c_{I I} \cdot v_{5}+\sqrt{c_{I I}^{2}-4 b_{I I}} \cdot v_{5}-c_{I I} \cdot v_{6}-\sqrt{c_{I I}^{2}-4 b_{I I}} \cdot v_{6}\right]$. 
$\cdot\left[\frac{1}{4}\left(2 v_{2}-\left(2 b_{I}+\sqrt{1-4 b_{I}}-1\right) v_{3}+\left(-2 b_{I}+\sqrt{1-4 b_{I}}+1\right) v_{4}\right)\right.$.

$\left.\left.\cdot\left[2 v_{1}+\left(c_{I I}\left(c_{I I}-\sqrt{c_{I I}^{2}-4 b_{I I}}\right)-2 b_{I I}\right) v_{5}+\left(c_{I I}\left(c_{I I}+\sqrt{c_{I I}^{2}-4 b_{I I}}\right)-2 b_{I I}\right) v_{6}\right]+1\right]\right\}$

- $f_{2}=\frac{1}{2} \mu_{I}\left[-2 v_{2}+\left(-\sqrt{1-4 b_{I}} \cdot a_{I}+a_{I}+2 b_{I}+\sqrt{1-4 b_{I}}-1\right) v_{3}-\right.$ $\left.-\left(\sqrt{1-4 b_{I}} \cdot a_{I}+a_{I}+2 b_{I}-\sqrt{1-4 b_{I}}-1\right) v_{4}\right]$

- $f_{3}=-\frac{1}{4 b_{I} \sqrt{1-4 b_{I}}}\left(\sqrt{1-4 b_{I}}+1\right) \mu_{I}\left\{2 v_{2}+\left[a_{I}\left(\sqrt{1-4 b_{I}}-1\right)-2 b_{I}-\right.\right.$ $\left.\left.-\sqrt{1-4 b_{I}}+1\right] v_{3}-\left(\sqrt{1-4 b_{I}} \cdot a_{I}+a_{I}+2 b_{I}-\sqrt{1-4 b_{I}}-1\right) v_{4}\right\}$

- $f_{4}=-\frac{1}{4 b_{I} \sqrt{1-4 b_{I}}}\left(\sqrt{1-4 b_{I}}-1\right) \mu_{I}\left\{2 v_{2}+\left[a_{I}\left(\sqrt{1-4 b_{I}}-1\right)-2 b_{I}-\right.\right.$ $\left.\left.-\sqrt{1-4 b_{I}}+1\right] v_{3}-\left(\sqrt{1-4 b_{I}} \cdot a_{I}+a_{I}+2 b_{I}-\sqrt{1-4 b_{I}}-1\right) v_{4}\right\}$

- $f_{5}=\frac{\mu_{I I}\left(c_{I I}+\sqrt{c_{I I}^{2}-4 b_{I I}}\right)}{4 b_{I I} \sqrt{c_{I I}^{2}-4 b_{I I}}}\left\{2\left(-v_{1}+v_{2}\right)-\left(2 b_{I}+\sqrt{1-4 b_{I}}-1\right) v_{3}+\right.$ $+\left(-2 b_{I}+\sqrt{1-4 b_{I}}+1\right) v_{4}+\left[2 b_{I I}+c_{I I}\left(\sqrt{c_{I I}^{2}-4 b_{I I}}-c_{I I}\right)\right] v_{5}-$ $-\left[c_{I I}\left(c_{I I}+\sqrt{c_{I I}^{2}-4 b_{I I}}\right)-2 b_{I I}\right] v_{6}-a_{I I}\left[-\left(\sqrt{1-4 b_{I}}-1\right) v_{3}+\right.$ $\left.+\left(\sqrt{1-4 b_{I}}+1\right) v_{4}-c_{I I} \cdot v_{5}+\sqrt{c_{I I}^{2}-4 b_{I I}} \cdot v_{5}-c_{I I} \cdot v_{6}-\sqrt{c_{I I}^{2}-4 b_{I I}} \cdot v_{6}\right]$. $\cdot\left[\frac{1}{4}\left(2 v_{2}-\left(2 b_{I}+\sqrt{1-4 b_{I}}-1\right) v_{3}+\left(-2 b_{I}+\sqrt{1-4 b_{I}}+1\right) v_{4}\right)\right.$. $\left.\left.\cdot\left[2 v_{1}+\left(c_{I I}\left(c_{I I}-\sqrt{c_{I I}^{2}-4 b_{I I}}\right)-2 b_{I I}\right) v_{5}+\left(c_{I I}\left(c_{I I}+\sqrt{c_{I I}^{2}-4 b_{I I}}\right)-2 b_{I I}\right) v_{6}\right]+1\right]\right\}$

- $f_{6}=-\frac{\mu_{I I}\left(c_{I I}-\sqrt{c_{I I}^{2}-4 b_{I I}}\right)}{4 b_{I I} \sqrt{c_{I I}^{2}-4 b_{I I}}}\left\{2\left(-v_{1}+v_{2}\right)-\left(2 b_{I}+\sqrt{1-4 b_{I}}-1\right) v_{3}+\right.$ $+\left(-2 b_{I}+\sqrt{1-4 b_{I}}+1\right) v_{4}+\left[-2 b_{I I}+c_{I I}\left(\sqrt{c_{I I}^{2}-4 b_{I I}}-c_{I I}\right)\right] v_{5}-$ $-\left[c_{I I}\left(c_{I I}+\sqrt{c_{I I}^{2}-4 b_{I I}}\right)-2 b_{I I}\right] v_{6}-a_{I I}\left[-\left(\sqrt{1-4 b_{I}}-1\right) v_{3}+\right.$ $\left.+\left(\sqrt{1-4 b_{I}}+1\right) v_{4}-c_{I I} \cdot v_{5}+\sqrt{c_{I I}^{2}-4 b_{I I}} \cdot v_{5}-c_{I I} \cdot v_{6}-\sqrt{c_{I I}^{2}-4 b_{I I}} \cdot v_{6}\right]$. 


$$
\begin{aligned}
& \cdot\left[\frac{1}{4}\left(2 v_{2}-\left(2 b_{I}+\sqrt{1-4 b_{I}}-1\right) v_{3}+\left(-2 b_{I}+\sqrt{1-4 b_{I}}+1\right) v_{4}\right) \cdot\right. \\
& \left.\left.\cdot\left[2 v_{1}+\left(c_{I I}\left(c_{I I}-\sqrt{c_{I I}^{2}-4 b_{I I}}\right)-2 b_{I I}\right) v_{5}+\left(c_{I I}\left(c_{I I}+\sqrt{c_{I I}^{2}-4 b_{I I}}\right)-2 b_{I I}\right) v_{6}\right]+1\right]\right\}
\end{aligned}
$$

Então, de acordo com o Teorema da Variedade Central (Guckenheimer e Holmes, 1983; Wiggins, 1990; Monteiro, 2002), procura-se a variedade central $W^{C}$ que seja tangente ao respectivo subespaço central $E^{C}$ no ponto de equilíbrio $P_{1}$. Assim, para $y, z, \mu_{I}$ e $\mu_{I I}$ suficientemente pequenos, tem-se:

$$
\begin{aligned}
W^{C}(0)=\{ & \left(v_{1}, v_{2}, v_{3}, v_{4}, v_{5}, v_{6}, \mu_{I}, \mu_{I I}\right) \in \mathfrak{R}^{4} / v_{1}=y, v_{2}=z, \\
& v_{3}=h_{1}\left(y, z, \mu_{I}, \mu_{I I}\right), v_{4}=h_{2}\left(y, z, \mu_{I}, \mu_{I I}\right), \\
& v_{5}=h_{3}\left(y, z, \mu_{I}, \mu_{I I}\right), v_{6}=h_{4}\left(y, z, \mu_{I}, \mu_{I I}\right), \\
& \left.h_{j}(0,0,0,0)=0, D h_{j}(0,0,0,0)=0, j=1,2,3,4\right\}
\end{aligned}
$$

Considere-se então, os seguintes polinômios estimadores $h_{1}\left(y, z, \mu_{I}, \mu_{I I}\right)$, $h_{2}\left(y, z, \mu_{I}, \mu_{I I}\right), h_{3}\left(y, z, \mu_{I}, \mu_{I I}\right)$ e $h_{4}\left(y, z, \mu_{I}, \mu_{I I}\right)$ compostos por monômios do segundo grau:

$$
\begin{aligned}
& h_{1}\left(y, z, \mu_{I}, \mu_{I I}\right)=\alpha_{1} \cdot y^{2}+\alpha_{2} \cdot z^{2}+\alpha_{3} \cdot \mu_{I}^{2}+\alpha_{4} \cdot \mu_{I I}^{2}+\alpha_{5} \cdot y \cdot z+\alpha_{6} \cdot y \cdot \mu_{I}+ \\
& +\alpha_{7} \cdot y \cdot \mu_{I I}+\alpha_{8} \cdot z \cdot \mu_{I}+\alpha_{9} \cdot z \cdot \mu_{I I}+\alpha_{10} \cdot \mu_{I} \cdot \mu_{I I},\left(\alpha_{j} \in \mathbb{R}, j=1, \ldots, 10\right) \\
& h_{2}\left(y, z, \mu_{I}, \mu_{I I}\right)=\beta_{1} \cdot y^{2}+\beta_{2} \cdot z^{2}+\beta_{3} \cdot \mu_{I}^{2}+\beta_{4} \cdot \mu_{I I}^{2}+\beta_{5} \cdot y \cdot z+\beta_{6} \cdot y \cdot \mu_{I}+ \\
& +\beta_{7} \cdot y \cdot \mu_{I I}+\beta_{8} \cdot z \cdot \mu_{I}+\beta_{9} \cdot z \cdot \mu_{I I}+\beta_{10} \cdot \mu_{I} \cdot \mu_{I I},\left(\beta_{j} \in \mathbb{R}, j=1, \ldots, 10\right) \\
& h_{3}\left(y, z, \mu_{I}, \mu_{I I}\right)=\gamma_{1} \cdot y^{2}+\gamma_{2} \cdot z^{2}+\gamma_{3} \cdot \mu_{I}^{2}+\gamma_{4} \cdot \mu_{I I}^{2}+\gamma_{5} \cdot y \cdot z+\gamma_{6} \cdot y \cdot \mu_{I}+ \\
& +\gamma_{7} \cdot y \cdot \mu_{I I}+\gamma_{8} \cdot z \cdot \mu_{I}+\gamma_{9} \cdot z \cdot \mu_{I I}+\gamma_{10} \cdot \mu_{I} \cdot \mu_{I I},\left(\gamma_{j} \in \mathbb{R}, j=1, \ldots, 10\right) \\
& h_{4}\left(y, z, \mu_{I}, \mu_{I I}\right)=\theta_{1} \cdot y^{2}+\theta_{2} \cdot z^{2}+\theta_{3} \cdot \mu_{I}^{2}+\theta_{4} \cdot \mu_{I I}^{2}+\theta_{5} \cdot y \cdot z+\theta_{6} \cdot y \cdot \mu_{I}+ \\
& +\theta_{7} \cdot y \cdot \mu_{I I}+\theta_{8} \cdot z \cdot \mu_{I}+\theta_{9} \cdot z \cdot \mu_{I I}+\theta_{10} \cdot \mu_{I} \cdot \mu_{I I},\left(\theta_{j} \in \mathbb{R}, j=1, \ldots, 10\right)
\end{aligned}
$$

Pelo teorema de Carr (Carr, 1981; Guckenheimer e Holmes, 1983; Wiggins, 1990; Monteiro, 2002), cada variedade central $W^{c}$ deve satisfazer $N\left(h\left(y, z, \mu_{I}, \mu_{I I}\right)\right)=0$. Portanto, tem-se: 


$$
\begin{aligned}
& \frac{\partial h_{1}\left(y, z, \mu_{I}, \mu_{I I}\right)}{\partial y}\left[\lambda_{1} \cdot y+f_{1}\left(y, z, h_{1}, h_{2}, h_{3}, h_{4}, \mu_{I}, \mu_{I I}\right)\right]+ \\
& +\frac{\partial h_{1}\left(y, z, \mu_{I}, \mu_{I I}\right)}{\partial z}\left[\lambda_{2} \cdot z+f_{2}\left(y, z, h_{1}, h_{2}, h_{3}, h_{4}, \mu_{I}, \mu_{I I}\right)\right]- \\
& -\lambda_{3} \cdot h_{1}\left(y, z, \mu_{I}, \mu_{I I}\right)-f_{3}\left(y, z, h_{1}, h_{2}, h_{3}, h_{4}, \mu_{I}, \mu_{I I}\right)=0 \\
& \frac{\partial h_{2}\left(y, z, \mu_{I}, \mu_{I I}\right)}{\partial y}\left[\lambda_{1} \cdot y+f_{1}\left(y, z, h_{1}, h_{2}, h_{3}, h_{4}, \mu_{I}, \mu_{I I}\right)\right]+ \\
& +\frac{\partial h_{2}\left(y, z, \mu_{I}, \mu_{I I}\right)}{\partial z}\left[\lambda_{2} \cdot z+f_{2}\left(y, z, h_{1}, h_{2}, h_{3}, h_{4}, \mu_{I}, \mu_{I I}\right)\right]- \\
& -\lambda_{4} \cdot h_{2}\left(y, z, \mu_{I}, \mu_{I I}\right)-f_{4}\left(y, z, h_{1}, h_{2}, h_{3}, h_{4}, \mu_{I}, \mu_{I I}\right)=0 \\
& \frac{\partial h_{3}\left(y, z, \mu_{I}, \mu_{I I}\right)}{\partial y}\left[\lambda_{1} \cdot y+f_{1}\left(y, z, h_{1}, h_{2}, h_{3}, h_{4}, \mu_{I}, \mu_{I I}\right)\right]+ \\
& +\frac{\partial h_{3}\left(y, z, \mu_{I}, \mu_{I I}\right)}{\partial z}\left[\lambda_{2} \cdot z+f_{2}\left(y, z, h_{1}, h_{2}, h_{3}, h_{4}, \mu_{I}, \mu_{I I}\right)\right]- \\
& -\lambda_{5} \cdot h_{3}\left(y, z, \mu_{I}, \mu_{I I}\right)-f_{5}\left(y, z, h_{1}, h_{2}, h_{3}, h_{4}, \mu_{I}, \mu_{I I}\right)=0 \\
& \frac{\partial h_{4}\left(y, z, \mu_{I}, \mu_{I I}\right)}{\partial y}\left[\lambda_{1} \cdot y+f_{1}\left(y, z, h_{1}, h_{2}, h_{3}, h_{4}, \mu_{I}, \mu_{I I}\right)\right]+ \\
& +\frac{\partial h_{4}\left(y, z, \mu_{I}, \mu_{I I}\right)}{\partial z}\left[\lambda_{2} \cdot z+f_{2}\left(y, z, h_{1}, h_{2}, h_{3}, h_{4}, \mu_{I}, \mu_{I I}\right)\right]- \\
& -\lambda_{6} \cdot h_{4}\left(y, z, \mu_{I}, \mu_{I I}\right)-f_{6}\left(y, z, h_{1}, h_{2}, h_{3}, h_{4}, \mu_{I}, \mu_{I I}\right)=0
\end{aligned}
$$

Utilizando (4.13) e (4.15) em (4.16) é possível obter, igualando-se os termos de igual potência, os coeficientes de $h_{1}\left(y, z, \mu_{I}, \mu_{I I}\right), h_{2}\left(y, z, \mu_{I}, \mu_{I I}\right), h_{3}\left(y, z, \mu_{I}, \mu_{I I}\right)$ e $h_{4}\left(y, z, \mu_{I}, \mu_{I I}\right):$

$$
\begin{aligned}
& y^{2}: \alpha_{1}=0, \beta_{1}=0, \gamma_{1}=0, \theta_{1}=0 \\
& z^{2}: \alpha_{2}=0, \beta_{2}=0, \gamma_{2}=0, \theta_{2}=0 \\
& \mu_{I}^{2}: \alpha_{3}=0, \beta_{3}=0, \gamma_{3}=0, \theta_{3}=0 \\
& \mu_{I I}^{2}: \alpha_{4}=0, \beta_{4}=0, \gamma_{4}=0, \theta_{4}=0 \\
& y \cdot z: \alpha_{5}=0, \beta_{5}=0, \gamma_{5}=0, \theta_{5}=0 \\
& y \cdot \mu_{I}: \alpha_{6}=0, \beta_{6}=0, \gamma_{6}=0, \theta_{6}=0
\end{aligned}
$$




$$
\begin{aligned}
& y \cdot \mu_{I I}: \alpha_{7}=0, \beta_{7}=0, \gamma_{7}=\frac{\sqrt{c_{I I}^{2}-4 b_{I I}}}{c_{I I}^{2}-4 b_{I I}}, \theta_{7}=\frac{\sqrt{c_{I I}^{2}-4 b_{I I}}}{4 b_{I I}-c_{I I}^{2}} \\
& z \cdot \mu_{I}: \alpha_{8}=\frac{\sqrt{1-4 b_{I}}}{4 b_{I}-1}, \beta_{6}=-\frac{\sqrt{1-4 b_{I}}}{4 b_{I}-1}, \gamma_{8}=0, \theta_{8}=0 \\
& z \cdot \mu_{I I}: \alpha_{9}=0, \beta_{9}=0, \gamma_{9}=\frac{\sqrt{c_{I I}^{2}-4 b_{I I}}}{4 b_{I I}-c_{I I}^{2}}, \theta_{9}=\frac{\sqrt{c_{I I}^{2}-4 b_{I I}}}{c_{I I}^{2}-4 b_{I I}} \\
& \mu_{I} \cdot \mu_{I I}: \alpha_{10}=0, \beta_{10}=0, \gamma_{10}=0, \theta_{10}=0
\end{aligned}
$$

Logo, os polinômios estimadores são:

$$
\begin{aligned}
& h_{1}\left(y, z, \mu_{I}, \mu_{I I}\right)=\frac{\sqrt{1-4 b_{I}}}{4 b_{I}-1} \cdot z \cdot \mu_{I} \\
& h_{2}\left(y, z, \mu_{I}, \mu_{I I}\right)=-\frac{\sqrt{1-4 b_{I}}}{4 b_{I}-1} \cdot z \cdot \mu_{I} \\
& h_{3}\left(y, z, \mu_{I}, \mu_{I I}\right)=\frac{\sqrt{c_{I I}^{2}-4 b_{I I}}}{c_{I I}^{2}-4 b_{I I}} \cdot y \cdot \mu_{I I}+\frac{\sqrt{c_{I I}^{2}-4 b_{I I}}}{4 b_{I I}-c_{I I}^{2}} \cdot z \cdot \mu_{I I} \\
& h_{4}\left(y, z, \mu_{I}, \mu_{I I}\right)=\frac{\sqrt{c_{I I}^{2}-4 b_{I I}}}{4 b_{I I}-c_{I I}^{2}} \cdot y \cdot \mu_{I I}+\frac{\sqrt{c_{I I}^{2}-4 b_{I I}}}{c_{I I}^{2}-4 b_{I I}} \cdot z \cdot \mu_{I I}
\end{aligned}
$$

Substituindo (4.18) no sistema (4.13), pode-se obter, desprezando os termos de ordem superior, o campo de vetores restrito à variedade central:

$$
\left\{\begin{array}{l}
\dot{y}=-\mu_{I I} \cdot y+\mu_{I I} \cdot z \\
\dot{z}=-\mu_{I} \cdot z \\
\dot{\mu}_{I}=0 \\
\dot{\mu}_{I I}=0
\end{array}\right.
$$

Para obter o ponto de equilíbrio de (4.19), basta fazer:

$$
\left\{\begin{array}{c}
\dot{y}=0 \Rightarrow-\mu_{I I} \cdot y^{*}+\mu_{I I} \cdot z^{*}=0 \\
\dot{z}=0 \Rightarrow-\mu_{I} \cdot z^{*}=0 \Rightarrow z^{*}=0
\end{array} \Rightarrow y^{*}=0 \Rightarrow E=\left(y^{*}, z^{*}\right)=(0,0)\right.
$$


A matriz Jacobiana de (4.19), calculada no ponto de equilíbrio $E=(0,0)$ é:

$$
J_{E}=\left[\begin{array}{cc}
-\mu_{I I} & +\mu_{I I} \\
0 & -\mu_{I}
\end{array}\right]
$$

Os autovalores de (4.21) são:

- $\lambda_{1}=-\mu_{I}$

- $\lambda_{2}=-\mu_{I I}$

De (4.22) se conclui que:

- Para $\mu_{I}<0$ e $\mu_{I I}>0$ o ponto de equilíbrio é instável.

- Para $\mu_{I}<0$ e $\mu_{I I}<0$ o ponto de equilíbrio é instável.

- Para $\mu_{I}>0$ e $\mu_{I I}<0$ o ponto de equilíbrio é instável.

- Para $\mu_{I}>0$ e $\mu_{I I}>0$ o ponto de equilíbrio é assintoticamente estável.

No capítulo VI serão realizadas conclusões mais detalhadas a respeito das expressões obtidas em (4.19), que tratam da estabilidade dos pontos de (4.23).

\subsubsection{ANÁLISE DA ESTABILIDADE DE $P_{2}=(-\pi, 0,0,0,0,0)$ VIA TVC}

Para a aplicação do TVC, faz-se necessária a expansão em série de Taylor das funções seno e co-seno em torno de $x_{1}^{*}=-\pi$ e $x_{4}^{*}=0$ :

$$
\operatorname{sen} x_{1} \approx-\left(x_{1}+\pi\right)+\ldots, \cos x_{1} \approx-1+\ldots, \text { sen } x_{4} \approx x_{4}+\ldots \text { e } \cos x_{4} \approx 1+\ldots
$$

E, portanto, a partir de (4.24), tem-se:

- $\cos \left(x_{4}-x_{1}\right)=\cos x_{4} \cos x_{1}+\operatorname{sen} x_{4} \operatorname{sen} x_{1}=-1-x_{4}\left(x_{1}+\pi\right)$

- $\operatorname{sen}\left(x_{4}-x_{1}\right)=\operatorname{sen} x_{4} \cos x_{1}-\operatorname{sen} x_{1} \cos x_{4}=-x_{4}+\left(x_{1}+\pi\right)$

Entretanto, o TVC exige que o ponto de equilíbrio seja a origem. Seja então a seguinte mudança de coordenadas:

$$
\bar{x}_{1}=x_{1}+\pi, \bar{x}_{2}=x_{2}, \bar{x}_{3}=x_{3}, \bar{x}_{4}=x_{4}, \bar{x}_{5}=x_{5} \text { e } \bar{x}_{6}=x_{6}
$$


Utilizando as expressões de (4.25), reescreve-se o sistema (5.8), já transladado para a origem de acordo com (4.26):

$$
\begin{aligned}
& \left\{\begin{array}{l}
\dot{\bar{x}}_{1}=\bar{x}_{2} \\
\dot{\bar{x}}_{2}=\bar{x}_{3} \\
\dot{\bar{x}}_{3}=-\frac{c_{I}}{b_{I}} \bar{x}_{3}-\frac{1}{b_{I}} \bar{x}_{2}-\frac{\mu_{I} \cdot a_{I}}{b_{I}} \bar{x}_{2}(-1)-\frac{\mu_{I}}{b_{I}}\left(-\bar{x}_{1}\right) \\
\dot{\bar{x}}_{4}=\bar{x}_{5} \\
\dot{\bar{x}}_{5}=\bar{x}_{6} \\
\dot{\bar{x}}_{6}=-\frac{c_{I I}}{b_{I I}} \bar{x}_{6}-\frac{1}{b_{I I}} \bar{x}_{5}-\frac{\mu_{I I} \cdot a_{I I}}{b_{I I}}\left(\bar{x}_{5}-\bar{x}_{2}\right)\left(-1-\bar{x}_{1} \cdot \bar{x}_{4}\right)-\frac{\mu_{I I}}{b_{I I}}\left(-\bar{x}_{4}+\bar{x}_{1}\right)
\end{array}\right. \\
& \dot{\mu}_{I}=0 \text { e } \dot{\mu}_{I I}=0
\end{aligned}
$$

O sistema (4.27) pode ser representado matricialmente como $\dot{\bar{X}}=J_{P_{1,2}} \cdot \bar{X}+\bar{F}$, $\dot{\mu}_{I}=0$ e $\dot{\mu}_{I I}=0$, ou seja:

$$
\begin{aligned}
& {\left[\begin{array}{c}
\dot{\bar{x}}_{1} \\
\dot{\bar{x}}_{2} \\
\dot{\bar{x}}_{3} \\
\dot{\bar{x}}_{4} \\
\dot{\bar{x}}_{5} \\
\dot{\bar{x}}_{6}
\end{array}\right]=\left[\begin{array}{cccccc}
0 & 1 & 0 & 0 & 0 & 0 \\
0 & 0 & 1 & 0 & 0 & 0 \\
0 & -\frac{1}{b_{I}} & -\frac{c_{I}}{b_{I}} & 0 & 0 & 0 \\
0 & 0 & 0 & 0 & 1 & 0 \\
0 & 0 & 0 & 0 & 0 & 1 \\
0 & 0 & 0 & 0 & -\frac{1}{b_{I I}} & -\frac{c_{I I}}{b_{I I}}
\end{array}\right] \cdot\left[\begin{array}{c}
\bar{x}_{1} \\
\bar{x}_{2} \\
\bar{x}_{3} \\
\bar{x}_{4} \\
\bar{x}_{5} \\
\bar{x}_{6}
\end{array}\right]+} \\
& +\left[\begin{array}{c}
0 \\
+\frac{\mu_{I} \cdot a_{I}}{b_{I}} \bar{x}_{2}+\frac{\mu_{I}}{b_{I}} \bar{x}_{1} \\
0 \\
\dot{\mu}_{I}=0 \mathrm{e} \dot{\mu}_{I I}=0 \quad \frac{\mu_{I I} \cdot a_{I I}}{b_{I I}}\left(\bar{x}_{5}-\bar{x}_{2}\right)\left(-1-\bar{x}_{4} \cdot \bar{x}_{1}\right)-\frac{\mu_{I I}}{b_{I I}}\left(\bar{x}_{1}-\bar{x}_{4}\right)
\end{array}\right]
\end{aligned}
$$


Conforme requerido pelo TVC, o sistema (4.28) precisa ser reescrito na forma canônica de Jordan. Para isso, define-se um novo conjunto de variáveis de estado $V=\left(v_{1}, v_{2}, v_{3}, v_{4}, v_{5}, v_{6}\right)^{T}$ de modo que $\bar{X}=M \cdot V$, sendo $M$ uma base composta pelos autovetores de $J_{P_{1,2,3,4}}$ de (4.9). Dessa forma, é possível dizer que $V=\left[M^{-1} \cdot J_{P_{1,2}} \cdot M\right] \cdot V+M^{-1} \cdot \bar{F}$, ou seja:

$$
\begin{aligned}
& {\left[\begin{array}{l}
\dot{v}_{1} \\
\dot{v}_{2} \\
\dot{v}_{3} \\
\dot{v}_{4} \\
\dot{v}_{5} \\
\dot{v}_{6}
\end{array}\right]=\left[\begin{array}{cccccc}
\lambda_{1} & 0 & 0 & 0 & 0 & 0 \\
0 & \lambda_{2} & 0 & 0 & 0 & 0 \\
0 & 0 & \lambda_{3} & 0 & 0 & 0 \\
0 & 0 & 0 & \lambda_{4} & 0 & 0 \\
0 & 0 & 0 & 0 & \lambda_{5} & 0 \\
0 & 0 & 0 & 0 & 0 & \lambda_{6}
\end{array}\right] \cdot\left[\begin{array}{c}
v_{1} \\
v_{2} \\
v_{3} \\
v_{4} \\
v_{5} \\
v_{6}
\end{array}\right]+\left[\begin{array}{c}
f_{1} \\
f_{2} \\
f_{3} \\
f_{4} \\
f_{5} \\
f_{6}
\end{array}\right]} \\
& \dot{\mu}_{I}=0 \text { e } \dot{\mu}_{I I}=0
\end{aligned}
$$

Em (4.29), tem-se que:

- $\lambda_{1}=0$

- $\lambda_{2}=0$

- $\lambda_{3}=-\frac{\left(c_{I}+\sqrt{c_{I}^{2}-4 b_{I}}\right)}{2 b_{I}}$

- $\lambda_{4}=\frac{\left(\sqrt{c_{I}^{2}-4 b_{I}}-c_{I}\right)}{2 b_{I}}$

- $\lambda_{5}=-\frac{\left(c_{I I}+\sqrt{c_{I I}^{2}-4 b_{I I}}\right)}{2 b_{I I}}$

- $\lambda_{6}=\frac{\left(\sqrt{c_{I I}^{2}-4 b_{I I}}-c_{I I}\right)}{2 b_{I I}}$

- $f_{1}=\frac{1}{2} \mu_{I I}\left\{2\left(v_{1}-v_{2}\right)-\left(-2 b_{I}+\sqrt{1-4 b_{I}}+1\right) v_{3}+\left(2 b_{I}+\sqrt{1-4 b_{I}}-1\right) v_{4}+\right.$ 


$$
\begin{aligned}
& +\left[c_{I I}\left(c_{I I}+\sqrt{c_{I I}^{2}-4 b_{I I}}\right)-2 b_{I I}\right] v_{5}+\left[c_{I I}\left(c_{I I}+\sqrt{c_{I I}^{2}-4 b_{I I}}\right)-2 b_{I I}\right] v_{6}- \\
& -a_{I I}\left[-\left(\sqrt{1-4 b_{I}}+1\right) v_{3}+\left(\sqrt{1-4 b_{I}}-1\right) v_{4}-\right. \\
& \left.-c_{I I} \cdot v_{5}+\sqrt{c_{I I}^{2}-4 b_{I I}} \cdot v_{5}-c_{I I} \cdot v_{6}-\sqrt{c_{I I}^{2}-4 b_{I I}} \cdot v_{6}\right] . \\
& \cdot\left[-\frac{1}{4}\left(2 v_{2}+\left(-2 b_{I}+\sqrt{1-4 b_{I}}+1\right) v_{3}-\left(2 b_{I}+\sqrt{1-4 b_{I}}-1\right) v_{4}\right) \cdot\right. \\
& \left.\left.\cdot\left[2 v_{1}+\left(c_{I I}\left(c_{I I}-\sqrt{c_{I I}^{2}-4 b_{I I}}\right)-2 b_{I I}\right) v_{5}+\left(c_{I I}\left(c_{I I}+\sqrt{c_{I I}^{2}-4 b_{I I}}\right)-2 b_{I I}\right) v_{6}\right]-1\right]\right\}
\end{aligned}
$$

- $f_{2}=\frac{1}{2} \mu_{I}\left[2 v_{2}+\left(\sqrt{1-4 b_{I}} \cdot a_{I}+a_{I}-2 b_{I}+\sqrt{1-4 b_{I}}+1\right) v_{3}-\right.$

$$
\left.-\left(\sqrt{1-4 b_{I}} \cdot a_{I}-a_{I}+2 b_{I}+\sqrt{1-4 b_{I}}-1\right) v_{4}\right]
$$

- $f_{3}=-\frac{1}{4 b_{I} \sqrt{1-4 b_{I}}}\left(\sqrt{1-4 b_{I}}+1\right) \mu_{I}\left\{2 v_{2}+\left[a_{I}\left(\sqrt{1-4 b_{I}}+1\right)-2 b_{I}+\right.\right.$

$$
\left.\left.+\sqrt{1-4 b_{I}}+1\right] v_{3}-\left(\sqrt{1-4 b_{I}} \cdot a_{I}-a_{I}+2 b_{I}+\sqrt{1-4 b_{I}}-1\right) v_{4}\right\}
$$

- $f_{4}=\frac{1}{4 b_{I} \sqrt{1-4 b_{I}}}\left(\sqrt{1-4 b_{I}}+1\right) \mu_{I}\left\{2 v_{2}+\left[a_{I}\left(\sqrt{1-4 b_{I}}+1\right)-2 b_{I}+\right.\right.$

$$
\left.\left.+\sqrt{1-4 b_{I}}+1\right] v_{3}-\left(\sqrt{1-4 b_{I}} \cdot a_{I}-a_{I}+2 b_{I}+\sqrt{1-4 b_{I}}-1\right) v_{4}\right\}
$$

- $f_{5}=\frac{\mu_{I I}\left(c_{I I}+\sqrt{c_{I I}^{2}-4 b_{I I}}\right)}{4 b_{I I} \sqrt{c_{I I}^{2}-4 b_{I I}}}\left\{2\left(v_{1}-v_{2}\right)-\left(-2 b_{I}+\sqrt{1-4 b_{I}}+1\right) v_{3}+\right.$

$$
\begin{aligned}
& +\left(2 b_{I}+\sqrt{1-4 b_{I}}-1\right) v_{4}+\left[c_{I I}\left(c_{I I}-\sqrt{c_{I I}^{2}-4 b_{I I}}\right)-2 b_{I I}\right] v_{5}+ \\
& +\left[c_{I I}\left(c_{I I}+\sqrt{c_{I I}^{2}-4 b_{I I}}\right)-2 b_{I I}\right] v_{6}-a_{I I}\left[-\left(\sqrt{1-4 b_{I}}+1\right) v_{3}+\right.
\end{aligned}
$$$$
\left.+\left(\sqrt{1-4 b_{I}}-1\right) v_{4}-c_{I I} \cdot v_{5}+\sqrt{c_{I I}^{2}-4 b_{I I}} \cdot v_{5}-c_{I I} \cdot v_{6}-\sqrt{c_{I I}^{2}-4 b_{I I}} \cdot v_{6}\right] \text {. }
$$$$
\cdot\left[-\frac{1}{4}\left(2 v_{2}+\left(-2 b_{I}+\sqrt{1-4 b_{I}}+1\right) v_{3}-\left(2 b_{I}+\sqrt{1-4 b_{I}}-1\right) v_{4}\right)\right. \text {. }
$$$$
\left.\left.\cdot\left[2 v_{1}+\left(c_{I I}\left(c_{I I}-\sqrt{c_{I I}^{2}-4 b_{I I}}\right)-2 b_{I I}\right) v_{5}+\left(c_{I I}\left(c_{I I}+\sqrt{c_{I I}^{2}-4 b_{I I}}\right)-2 b_{I I}\right) v_{6}\right]-1\right]\right\}
$$

- $f_{6}=-\frac{\mu_{I I}\left(c_{I I}-\sqrt{c_{I I}^{2}-4 b_{I I}}\right)}{4 b_{I I} \sqrt{c_{I I}^{2}-4 b_{I I}}}\left\{2\left(v_{1}-v_{2}\right)-\left(-2 b_{I}+\sqrt{1-4 b_{I}}+1\right) v_{3}+\right.$ 


$$
\begin{aligned}
& +\left(2 b_{I}+\sqrt{1-4 b_{I}}-1\right) v_{4}+\left[c_{I I}\left(c_{I I}-\sqrt{c_{I I}^{2}-4 b_{I I}}\right)-2 b_{I I}\right] v_{5}+ \\
& +\left[c_{I I}\left(c_{I I}+\sqrt{c_{I I}^{2}-4 b_{I I}}\right)-2 b_{I I}\right] v_{6}-a_{I I}\left[-\left(\sqrt{1-4 b_{I}}+1\right) v_{3}+\right. \\
& \left.+\left(\sqrt{1-4 b_{I}}-1\right) v_{4}-c_{I I} \cdot v_{5}+\sqrt{c_{I I}^{2}-4 b_{I I}} \cdot v_{5}-c_{I I} \cdot v_{6}-\sqrt{c_{I I}^{2}-4 b_{I I}} \cdot v_{6}\right] \\
& \cdot\left[-\frac{1}{4}\left(2 v_{2}+\left(-2 b_{I}+\sqrt{1-4 b_{I}}+1\right) v_{3}-\left(2 b_{I}+\sqrt{1-4 b_{I}}-1\right) v_{4}\right) \cdot\right. \\
& \left.\left.\cdot\left[2 v_{1}+\left(c_{I I}\left(c_{I I}-\sqrt{c_{I I}^{2}-4 b_{I I}}\right)-2 b_{I I}\right) v_{5}+\left(c_{I I}\left(c_{I I}+\sqrt{c_{I I}^{2}-4 b_{I I}}\right)-2 b_{I I}\right) v_{6}\right]-1\right]\right\}
\end{aligned}
$$

Então, de acordo com o Teorema da Variedade Central (Guckenheimer e Holmes, 1983; Wiggins, 1990; Monteiro, 2002), procura-se a variedade central $W^{C}$ que seja tangente ao respectivo subespaço central $E^{C}$ no ponto de equilíbrio $P_{2}$. Assim, para $y, z, \mu_{I}$ e $\mu_{I I}$ suficientemente pequenos, tem-se:

$$
\begin{aligned}
W^{C}(0)=\{ & \left(v_{1}, v_{2}, v_{3}, v_{4}, v_{5}, v_{6}, \mu_{I}, \mu_{I I}\right) \in \mathfrak{R}^{4} / v_{1}=y, v_{2}=z, \\
& v_{3}=h_{1}\left(y, z, \mu_{I}, \mu_{I I}\right), v_{4}=h_{2}\left(y, z, \mu_{I}, \mu_{I I}\right), \\
& v_{5}=h_{3}\left(y, z, \mu_{I}, \mu_{I I}\right), v_{6}=h_{4}\left(y, z, \mu_{I}, \mu_{I I}\right), \\
& \left.h_{j}(0,0,0,0)=0, D h_{j}(0,0,0,0)=0, j=1,2,3,4\right\}
\end{aligned}
$$

Considere-se então, os seguintes polinômios estimadores $h_{1}\left(y, z, \mu_{I}, \mu_{I I}\right)$, $h_{2}\left(y, z, \mu_{I}, \mu_{I I}\right), h_{3}\left(y, z, \mu_{I}, \mu_{I I}\right)$ e $h_{4}\left(y, z, \mu_{I}, \mu_{I I}\right)$ compostos por monômios do segundo grau:

$$
\begin{gathered}
h_{1}\left(y, z, \mu_{I}, \mu_{I I}\right)=\alpha_{1} \cdot y^{2}+\alpha_{2} \cdot z^{2}+\alpha_{3} \cdot \mu_{I}^{2}+\alpha_{4} \cdot \mu_{I I}^{2}+\alpha_{5} \cdot y \cdot z+\alpha_{6} \cdot y \cdot \mu_{I}+ \\
\quad+\alpha_{7} \cdot y \cdot \mu_{I I}+\alpha_{8} \cdot z \cdot \mu_{I}+\alpha_{9} \cdot z \cdot \mu_{I I}+\alpha_{10} \cdot \mu_{I} \cdot \mu_{I I},\left(\alpha_{j} \in \mathbb{R}, j=1, \ldots, 10\right) \\
h_{2}\left(y, z, \mu_{I}, \mu_{I I}\right)=\beta_{1} \cdot y^{2}+\beta_{2} \cdot z^{2}+\beta_{3} \cdot \mu_{I}^{2}+\beta_{4} \cdot \mu_{I I}^{2}+\beta_{5} \cdot y \cdot z+\beta_{6} \cdot y \cdot \mu_{I}+ \\
\quad+\beta_{7} \cdot y \cdot \mu_{I I}+\beta_{8} \cdot z \cdot \mu_{I}+\beta_{9} \cdot z \cdot \mu_{I I}+\beta_{10} \cdot \mu_{I} \cdot \mu_{I I},\left(\beta_{j} \in \mathbb{R}, j=1, \ldots, 10\right) \\
h_{3}\left(y, z, \mu_{I}, \mu_{I I}\right)=\gamma_{1} \cdot y^{2}+\gamma_{2} \cdot z^{2}+\gamma_{3} \cdot \mu_{I}^{2}+\gamma_{4} \cdot \mu_{I I}^{2}+\gamma_{5} \cdot y \cdot z+\gamma_{6} \cdot y \cdot \mu_{I}+ \\
\quad+\gamma_{7} \cdot y \cdot \mu_{I I}+\gamma_{8} \cdot z \cdot \mu_{I}+\gamma_{9} \cdot z \cdot \mu_{I I}+\gamma_{10} \cdot \mu_{I} \cdot \mu_{I I},\left(\gamma_{j} \in \mathbb{R}, j=1, \ldots, 10\right) \\
h_{4}\left(y, z, \mu_{I}, \mu_{I I}\right)=\theta_{1} \cdot y^{2}+\theta_{2} \cdot z^{2}+\theta_{3} \cdot \mu_{I}^{2}+\theta_{4} \cdot \mu_{I I}^{2}+\theta_{5} \cdot y \cdot z+\theta_{6} \cdot y \cdot \mu_{I}+ \\
\quad+\theta_{7} \cdot y \cdot \mu_{I I}+\theta_{8} \cdot z \cdot \mu_{I}+\theta_{9} \cdot z \cdot \mu_{I I}+\theta_{10} \cdot \mu_{I} \cdot \mu_{I I},\left(\theta_{j} \in \mathbb{R}, j=1, \ldots, 10\right)
\end{gathered}
$$


Pelo teorema de Carr (Carr, 1981; Guckenheimer e Holmes, 1983; Wiggins, 1990; Monteiro, 2002), a variedade central $W^{c}$ deve satisfazer $N\left(h\left(y, z, \mu_{I}, \mu_{I I}\right)\right)=0$. Portanto, tem-se:

$$
\begin{aligned}
& \frac{\partial h_{1}\left(y, z, \mu_{I}, \mu_{I I}\right)}{\partial y}\left[\lambda_{1} \cdot y+f_{1}\left(y, z, h_{1}, h_{2}, h_{3}, h_{4}, \mu_{I}, \mu_{I I}\right)\right]+ \\
& +\frac{\partial h_{1}\left(y, z, \mu_{I}, \mu_{I I}\right)}{\partial z}\left[\lambda_{2} \cdot z+f_{2}\left(y, z, h_{1}, h_{2}, h_{3}, h_{4}, \mu_{I}, \mu_{I I}\right)\right]- \\
& -\lambda_{3} \cdot h_{1}\left(y, z, \mu_{I}, \mu_{I I}\right)-f_{3}\left(y, z, h_{1}, h_{2}, h_{3}, h_{4}, \mu_{I}, \mu_{I I}\right)=0 \\
& \frac{\partial h_{2}\left(y, z, \mu_{I}, \mu_{I I}\right)}{\partial y}\left[\lambda_{1} \cdot y+f_{1}\left(y, z, h_{1}, h_{2}, h_{3}, h_{4}, \mu_{I}, \mu_{I I}\right)\right]+ \\
& +\frac{\partial h_{2}\left(y, z, \mu_{I}, \mu_{I I}\right)}{\partial z}\left[\lambda_{2} \cdot z+f_{2}\left(y, z, h_{1}, h_{2}, h_{3}, h_{4}, \mu_{I}, \mu_{I I}\right)\right]- \\
& -\lambda_{4} \cdot h_{2}\left(y, z, \mu_{I}, \mu_{I I}\right)-f_{4}\left(y, z, h_{1}, h_{2}, h_{3}, h_{4}, \mu_{I}, \mu_{I I}\right)=0 \\
& \frac{\partial h_{3}\left(y, z, \mu_{I}, \mu_{I I}\right)}{\partial y}\left[\lambda_{1} \cdot y+f_{1}\left(y, z, h_{1}, h_{2}, h_{3}, h_{4}, \mu_{I}, \mu_{I I}\right)\right]+ \\
& +\frac{\partial h_{3}\left(y, z, \mu_{I}, \mu_{I I}\right)}{\partial z}\left[\lambda_{2} \cdot z+f_{2}\left(y, z, h_{1}, h_{2}, h_{3}, h_{4}, \mu_{I}, \mu_{I I}\right)\right]- \\
& -\lambda_{5} \cdot h_{3}\left(y, z, \mu_{I}, \mu_{I I}\right)-f_{5}\left(y, z, h_{1}, h_{2}, h_{3}, h_{4}, \mu_{I}, \mu_{I I}\right)=0 \\
& \frac{\partial h_{4}\left(y, z, \mu_{I}, \mu_{I I}\right)}{\partial y}\left[\lambda_{1} \cdot y+f_{1}\left(y, z, h_{1}, h_{2}, h_{3}, h_{4}, \mu_{I}, \mu_{I I}\right)\right]+ \\
& +\frac{\partial h_{4}\left(y, z, \mu_{I}, \mu_{I I}\right)}{\partial z}\left[\lambda_{2} \cdot z+f_{2}\left(y, z, h_{1}, h_{2}, h_{3}, h_{4}, \mu_{I}, \mu_{I I}\right)\right]- \\
& -\lambda_{6} \cdot h_{4}\left(y, z, \mu_{I}, \mu_{I I}\right)-f_{6}\left(y, z, h_{1}, h_{2}, h_{3}, h_{4}, \mu_{I}, \mu_{I I}\right)=0
\end{aligned}
$$

Utilizando (4.29) e (4.31) em (4.32) é possível obter, igualando-se os termos de igual potência, os coeficientes de $h_{1}\left(y, z, \mu_{I}, \mu_{I I}\right), h_{2}\left(y, z, \mu_{I}, \mu_{I I}\right), h_{3}\left(y, z, \mu_{I}, \mu_{I I}\right)$ e $h_{4}\left(y, z, \mu_{I}, \mu_{I I}\right)$ : 


$$
\begin{aligned}
& y^{2}: \alpha_{1}=0, \beta_{1}=0, \gamma_{1}=0, \theta_{1}=0 \\
& z^{2}: \alpha_{2}=0, \beta_{2}=0, \gamma_{2}=0, \theta_{2}=0 \\
& \mu_{I}^{2}: \alpha_{3}=0, \beta_{3}=0, \gamma_{3}=0, \theta_{3}=0 \\
& \mu_{I I}^{2}: \alpha_{4}=0, \beta_{4}=0, \gamma_{4}=0, \theta_{4}=0 \\
& y \cdot z: \alpha_{5}=0, \beta_{5}=0, \gamma_{5}=0, \theta_{5}=0 \\
& y \cdot \mu_{I}: \alpha_{6}=0, \beta_{6}=0, \gamma_{6}=0, \theta_{6}=0 \\
& y \cdot \mu_{I I}: \alpha_{7}=0, \beta_{7}=0, \gamma_{7}=\frac{\sqrt{c_{I I}^{2}-4 b_{I I}}}{c_{I I}^{2}-4 b_{I I}}, \theta_{7}=\frac{\sqrt{c_{I I}^{2}-4 b_{I I}}}{4 b_{I I}-c_{I I}^{2}} \\
& \mu_{I} \cdot \mu_{I I}: \alpha_{10}=0, \beta_{10}=0, \gamma_{10}=0, \theta_{10}=0 \\
& z \cdot \mu_{I I}: \alpha_{9}=0, \beta_{9}=0, \gamma_{9}=\frac{\sqrt{c_{I I}^{2}-4 b_{I I}}}{4 b_{I I}-c_{I I}^{2}}, \theta_{9}=\frac{\sqrt{c_{I I}^{2}-4 b_{I I}}}{c_{I I}^{2}-4 b_{I I}} \\
& 4 b_{I}-1
\end{aligned}
$$

Logo, os polinômios estimadores são:

$$
\begin{aligned}
& h_{1}\left(y, z, \mu_{I}, \mu_{I I}\right)=-\frac{\sqrt{1-4 b_{I}}}{4 b_{I}-1} \cdot z \cdot \mu_{I} \\
& h_{2}\left(y, z, \mu_{I}, \mu_{I I}\right)=+\frac{\sqrt{1-4 b_{I}}}{4 b_{I}-1} \cdot z \cdot \mu_{I} \\
& h_{3}\left(y, z, \mu_{I}, \mu_{I I}\right)=\frac{\sqrt{c_{I I}^{2}-4 b_{I I}}}{c_{I I}^{2}-4 b_{I I}} \cdot y \cdot \mu_{I I}+\frac{\sqrt{c_{I I}^{2}-4 b_{I I}}}{4 b_{I I}-c_{I I}^{2}} \cdot z \cdot \mu_{I I} \\
& h_{4}\left(y, z, \mu_{I}, \mu_{I I}\right)=\frac{\sqrt{c_{I I}^{2}-4 b_{I I}}}{4 b_{I I}-c_{I I}^{2}} \cdot y \cdot \mu_{I I}+\frac{\sqrt{c_{I I}^{2}-4 b_{I I}}}{c_{I I}^{2}-4 b_{I I}} \cdot z \cdot \mu_{I I}
\end{aligned}
$$


Substituindo (4.34) no sistema (4.29), pode-se obter, desprezando os termos de ordem superior, o campo de vetores restrito à variedade central:

$$
\left\{\begin{array}{l}
\dot{y}=+\mu_{I I} \cdot y-\mu_{I I} \cdot z \\
\dot{z}=+\mu_{I} \cdot z \\
\dot{\mu}_{I}=0 \\
\dot{\mu}_{I I}=0
\end{array}\right.
$$

Para obter o ponto de equilíbrio de (4.35), basta fazer:

$$
\left\{\begin{array}{c}
\dot{y}=0 \Rightarrow+\mu_{I I} \cdot y^{*}-\mu_{I I} \cdot z^{*}=0 \\
\dot{z}=0 \Rightarrow+\mu_{I} \cdot z^{*}=0 \Rightarrow z^{*}=0
\end{array} \Rightarrow y^{*}=0 \Rightarrow E=\left(y^{*}, z^{*}\right)=(0,0)\right.
$$

A matriz Jacobiana de (4.35) calculada no ponto de equilíbrio $E=(0,0)$ é:

$$
J_{E}=\left[\begin{array}{cc}
+\mu_{I I} & -\mu_{I I} \\
0 & +\mu_{I}
\end{array}\right]
$$

Os autovalores de (4.37) são:

- $\lambda_{1}=+\mu_{I}$

- $\lambda_{2}=+\mu_{I I}$

De (4.38) se conclui que:

- Para $\mu_{I}>0$ e $\mu_{I I}>0$ o ponto de equilíbrio é instável.

- Para $\mu_{I}>0$ e $\mu_{I I}<0$ o ponto de equilíbrio é instável.

- Para $\mu_{I}<0$ e $\mu_{I I}>0$ o ponto de equilíbrio é instável.

- Para $\mu_{I}<0$ e $\mu_{I I}<0$ o ponto de equilíbrio é assintoticamente estável.

No capítulo VI serão realizadas conclusões mais detalhadas a respeito das expressões obtidas em (5.35), que tratam da estabilidade dos pontos de (4.39).

4.2.4 ANÁLISE DA ESTABILIDADE DE $P_{3}=(-\pi, 0,0,-\pi, 0,0)$ VIA TVC 
Para a aplicação do TVC, faz-se necessária a expansão em série de Taylor das funções seno e co-seno em torno de $x_{1}^{*}=-\pi$ e $x_{4}^{*}=-\pi$ :

$$
\begin{aligned}
& \operatorname{sen} x_{1} \approx-\left(x_{1}+\pi\right)+\ldots, \cos x_{1} \approx-1+\ldots \\
& \operatorname{sen} x_{4} \approx-\left(x_{4}+\pi\right)+\ldots \text { e } \cos x_{4} \approx-1+\ldots
\end{aligned}
$$

E, portanto, a partir de (4.40), tem-se:

- $\cos \left(x_{4}-x_{1}\right)=\cos x_{4} \cos x_{1}+\operatorname{sen} x_{4} \operatorname{sen} x_{1}=+1+\left(x_{4}+\pi\right)\left(x_{1}+\pi\right)$

- $\operatorname{sen}\left(x_{4}-x_{1}\right)=\operatorname{sen} x_{4} \cos x_{1}-\operatorname{sen} x_{1} \cos x_{4}=+\left(x_{4}+\pi\right)-\left(x_{1}+\pi\right)$

Entretanto, o TVC exige que o ponto de equilíbrio seja a origem. Seja então a seguinte mudança de coordenadas:

$$
\bar{x}_{1}=x_{1}+\pi, \bar{x}_{2}=x_{2}, \bar{x}_{3}=x_{3}, \bar{x}_{4}=x_{4}+\pi, \bar{x}_{5}=x_{5} \text { e } \bar{x}_{6}=x_{6}
$$

Utilizando as expressões de (4.41), reescreve-se o sistema (4.8), já transladado para a origem de acordo com (4.42):

$$
\begin{aligned}
& \left\{\begin{array}{l}
\dot{\bar{x}}_{1}=\bar{x}_{2} \\
\dot{\bar{x}}_{2}=\bar{x}_{3} \\
\dot{\bar{x}}_{3}=-\frac{c_{I}}{b_{I}} \bar{x}_{3}-\frac{1}{b_{I}} \bar{x}_{2}-\frac{\mu_{I} \cdot a_{I}}{b_{I}} \bar{x}_{2}(-1)-\frac{\mu_{I}}{b_{I}}\left(-\bar{x}_{1}\right) \\
\dot{\bar{x}}_{4}=\bar{x}_{5} \\
\dot{\bar{x}}_{5}=\bar{x}_{6} \\
\dot{\bar{x}}_{6}=-\frac{c_{I I}}{b_{I I}} \bar{x}_{6}-\frac{1}{b_{I I}} \bar{x}_{5}-\frac{\mu_{I I} \cdot a_{I I}}{b_{I I}}\left(\bar{x}_{5}-\bar{x}_{2}\right)\left(+1+\bar{x}_{1} \cdot \bar{x}_{4}\right)-\frac{\mu_{I I}}{b_{I I}}\left(+\bar{x}_{4}-\bar{x}_{1}\right)
\end{array}\right. \\
& \dot{\mu}_{I}=0 \text { e } \dot{\mu}_{I I}=0
\end{aligned}
$$

O sistema (4.43) pode ser representado matricialmente como $\dot{\bar{X}}=J_{P_{1,2}} \cdot \bar{X}+\bar{F}$, $\dot{\mu}_{I}=0$ e $\dot{\mu}_{I I}=0$, ou seja: 


$$
\begin{aligned}
& {\left[\begin{array}{c}
\dot{\bar{x}}_{1} \\
\dot{\bar{x}}_{2} \\
\dot{\bar{x}}_{3} \\
\dot{\bar{x}}_{4} \\
\dot{\bar{x}}_{5} \\
\dot{\bar{x}}_{6}
\end{array}\right]=\left[\begin{array}{cccccc}
0 & 1 & 0 & 0 & 0 & 0 \\
0 & 0 & 1 & 0 & 0 & 0 \\
0 & -\frac{1}{b_{I}} & -\frac{c_{I}}{b_{I}} & 0 & 0 & 0 \\
0 & 0 & 0 & 0 & 1 & 0 \\
0 & 0 & 0 & 0 & 0 & 1 \\
0 & 0 & 0 & 0 & -\frac{1}{b_{I I}} & -\frac{c_{I I}}{b_{I I}}
\end{array}\right] \cdot\left[\begin{array}{c}
\bar{x}_{1} \\
\bar{x}_{2} \\
\bar{x}_{3} \\
\bar{x}_{4} \\
\bar{x}_{5} \\
\bar{x}_{6}
\end{array}\right]+} \\
& +\left[\begin{array}{c}
0 \\
+\frac{\mu_{I} \cdot a_{I}}{b_{I}} \bar{x}_{2}+\frac{\mu_{I}}{b_{I}} \bar{x}_{1} \\
0 \\
\dot{\mu}_{I}=0 \\
=0 \\
\dot{\mu}_{I I}=0 \quad a_{I I}\left(\bar{x}_{5}-\bar{x}_{2}\right)\left(+1+\bar{x}_{4} \cdot \bar{x}_{1}\right)-\frac{\mu_{I I}}{b_{I I}}\left(\bar{x}_{4}-\bar{x}_{1}\right)
\end{array}\right]
\end{aligned}
$$

Conforme requerido pelo TVC, o sistema (4.44) precisa ser reescrito na forma canônica de Jordan. Para isso, define-se um novo conjunto de variáveis de estado $V=\left(v_{1}, v_{2}, v_{3}, v_{4}, v_{5}, v_{6}\right)^{T}$ de modo que $\bar{X}=M \cdot V$, sendo $M$ uma base composta pelos autovetores de $J_{P_{1,2,3,4}}$ de (4.9). Dessa forma, é possível dizer que $V=\left[M^{-1} \cdot J_{P_{1,2}} \cdot M\right] \cdot V+M^{-1} \cdot \bar{F}$, ou seja:

$$
\begin{aligned}
& {\left[\begin{array}{l}
\dot{v}_{1} \\
\dot{v}_{2} \\
\dot{v}_{3} \\
\dot{v}_{4} \\
\dot{v}_{5} \\
\dot{v}_{6}
\end{array}\right]=\left[\begin{array}{cccccc}
\lambda_{1} & 0 & 0 & 0 & 0 & 0 \\
0 & \lambda_{2} & 0 & 0 & 0 & 0 \\
0 & 0 & \lambda_{3} & 0 & 0 & 0 \\
0 & 0 & 0 & \lambda_{4} & 0 & 0 \\
0 & 0 & 0 & 0 & \lambda_{5} & 0 \\
0 & 0 & 0 & 0 & 0 & \lambda_{6}
\end{array}\right] \cdot\left[\begin{array}{c}
v_{1} \\
v_{2} \\
v_{3} \\
v_{4} \\
v_{5} \\
v_{6}
\end{array}\right]+\left[\begin{array}{c}
f_{1} \\
f_{2} \\
f_{3} \\
f_{4} \\
f_{5} \\
f_{6}
\end{array}\right]} \\
& \dot{\mu}_{I}=0 \text { e } \dot{\mu}_{I I}=0
\end{aligned}
$$

Em (4.45), tem-se que:

- $\lambda_{1}=0$ 
- $\lambda_{2}=0$

- $\lambda_{3}=-\frac{\left(c_{I}+\sqrt{c_{I}^{2}-4 b_{I}}\right)}{2 b_{I}}$

- $\lambda_{4}=\frac{\left(\sqrt{c_{I}^{2}-4 b_{I}}-c_{I}\right)}{2 b_{I}}$

- $\lambda_{5}=-\frac{\left(c_{I I}+\sqrt{c_{I I}^{2}-4 b_{I I}}\right)}{2 b_{I I}}$

- $\lambda_{6}=\frac{\left(\sqrt{c_{I I}^{2}-4 b_{I I}}-c_{I I}\right)}{2 b_{I I}}$

- $f_{1}=\frac{1}{2} \mu_{I I}\left\{2\left(-v_{1}+v_{2}\right)+\left(-2 b_{I}+\sqrt{1-4 b_{I}}+1\right) v_{3}-\left(2 b_{I}+\sqrt{1-4 b_{I}}-1\right) v_{4}+\right.$

$+\left[2 b_{I I}+c_{I I}\left(\sqrt{c_{I I}^{2}-4 b_{I I}}-c_{I I}\right)\right] v_{5}+\left[c_{I I}\left(c_{I I}+\sqrt{c_{I I}^{2}-4 b_{I I}}\right)-2 b_{I I}\right] v_{6}-$

$-a_{I I}\left[-\left(\sqrt{1-4 b_{I}}+1\right) v_{3}+\left(\sqrt{1-4 b_{I}}-1\right) v_{4}-\right.$

$\left.-c_{I I} \cdot v_{5}+\sqrt{c_{I I}^{2}-4 b_{I I}} \cdot v_{5}-c_{I I} \cdot v_{6}-\sqrt{c_{I I}^{2}-4 b_{I I}} \cdot v_{6}\right]$.

$\cdot\left[\frac{1}{4}\left(2 v_{2}+\left(-2 b_{I}+\sqrt{1-4 b_{I}}+1\right) v_{3}-\left(2 b_{I}+\sqrt{1-4 b_{I}}-1\right) v_{4}\right)\right.$.

$\left.\left.\cdot\left[2 v_{1}+\left(c_{I I}\left(c_{I I}-\sqrt{c_{I I}^{2}-4 b_{I I}}\right)-2 b_{I I}\right) v_{5}+\left(c_{I I}\left(c_{I I}+\sqrt{c_{I I}^{2}-4 b_{I I}}\right)-2 b_{I I}\right) v_{6}\right]+1\right]\right\}$

- $f_{2}=\frac{1}{2} \mu_{I}\left[2 v_{2}+\left(\sqrt{1-4 b_{I}} \cdot a_{I}+a_{I}-2 b_{I}+\sqrt{1-4 b_{I}}+1\right) v_{3}-\right.$ $\left.-\left(\sqrt{1-4 b_{I}} \cdot a_{I}-a_{I}+2 b_{I}+\sqrt{1-4 b_{I}}-1\right) v_{4}\right]$

- $f_{3}=\frac{1}{4 b_{I} \sqrt{1-4 b_{I}}}\left(\sqrt{1-4 b_{I}}-1\right) \mu_{I}\left\{2 v_{2}+\left[a_{I}\left(\sqrt{1-4 b_{I}}+1\right)-2 b_{I}+\right.\right.$ $\left.\left.+\sqrt{1-4 b_{I}}+1\right] v_{3}-\left(\sqrt{1-4 b_{I}} \cdot a_{I}-a_{I}+2 b_{I}+\sqrt{1-4 b_{I}}-1\right) v_{4}\right\}$

- $f_{4}=\frac{1}{4 b_{I} \sqrt{1-4 b_{I}}}\left(\sqrt{1-4 b_{I}}+1\right) \mu_{I}\left\{2 v_{2}+\left[a_{I}\left(\sqrt{1-4 b_{I}}+1\right)-2 b_{I}+\right.\right.$ $\left.\left.+\sqrt{1-4 b_{I}}+1\right] v_{3}-\left(\sqrt{1-4 b_{I}} \cdot a_{I}-a_{I}+2 b_{I}+\sqrt{1-4 b_{I}}-1\right) v_{4}\right\}$ 


$$
\begin{aligned}
& \text { - } f_{5}=\frac{\mu_{I I}\left(c_{I I}+\sqrt{c_{I I}^{2}-4 b_{I I}}\right)}{4 b_{I I} \sqrt{c_{I I}^{2}-4 b_{I I}}}\left\{2\left(-v_{1}+v_{2}\right)+\left(-2 b_{I}+\sqrt{1-4 b_{I}}+1\right) v_{3}-\right. \\
& -\left(2 b_{I}+\sqrt{1-4 b_{I}}-1\right) v_{4}+\left[2 b_{I I}+c_{I I}\left(\sqrt{c_{I I}^{2}-4 b_{I I}}-c_{I I}\right)\right] v_{5}- \\
& -\left[c_{I I}\left(c_{I I}+\sqrt{c_{I I}^{2}-4 b_{I I}}\right)-2 b_{I I}\right] v_{6}-a_{I I}\left[-\left(\sqrt{1-4 b_{I}}+1\right) v_{3}+\right. \\
& \left.+\left(\sqrt{1-4 b_{I}}-1\right) v_{4}-c_{I I} \cdot v_{5}+\sqrt{c_{I I}^{2}-4 b_{I I}} \cdot v_{5}-c_{I I} \cdot v_{6}-\sqrt{c_{I I}^{2}-4 b_{I I}} \cdot v_{6}\right] \text {. } \\
& \cdot\left[\frac{1}{4}\left(2 v_{2}+\left(-2 b_{I}+\sqrt{1-4 b_{I}}+1\right) v_{3}-\left(2 b_{I}+\sqrt{1-4 b_{I}}-1\right) v_{4}\right)\right. \text {. } \\
& \left.\left.\cdot\left[2 v_{1}+\left(c_{I I}\left(c_{I I}-\sqrt{c_{I I}^{2}-4 b_{I I}}\right)-2 b_{I I}\right) v_{5}+\left(c_{I I}\left(c_{I I}+\sqrt{c_{I I}^{2}-4 b_{I I}}\right)-2 b_{I I}\right) v_{6}\right]+1\right]\right\} \\
& \text { - } f_{6}=-\frac{\mu_{I I}\left(c_{I I}-\sqrt{c_{I I}^{2}-4 b_{I I}}\right)}{4 b_{I I} \sqrt{c_{I I}^{2}-4 b_{I I}}}\left\{2\left(-v_{1}+v_{2}\right)+\left(-2 b_{I}+\sqrt{1-4 b_{I}}+1\right) v_{3}-\right. \\
& -\left(2 b_{I}+\sqrt{1-4 b_{I}}-1\right) v_{4}+\left[2 b_{I I}+c_{I I}\left(\sqrt{c_{I I}^{2}-4 b_{I I}}-c_{I I}\right)-\right] v_{5}- \\
& -\left[c_{I I}\left(c_{I I}+\sqrt{c_{I I}^{2}-4 b_{I I}}\right)-2 b_{I I}\right] v_{6}-a_{I I}\left[-\left(\sqrt{1-4 b_{I}}+1\right) v_{3}+\right. \\
& \left.+\left(\sqrt{1-4 b_{I}}-1\right) v_{4}-c_{I I} \cdot v_{5}+\sqrt{c_{I I}^{2}-4 b_{I I}} \cdot v_{5}-c_{I I} \cdot v_{6}-\sqrt{c_{I I}^{2}-4 b_{I I}} \cdot v_{6}\right] \text {. } \\
& \cdot\left[\frac{1}{4}\left(2 v_{2}+\left(-2 b_{I}+\sqrt{1-4 b_{I}}+1\right) v_{3}-\left(2 b_{I}+\sqrt{1-4 b_{I}}-1\right) v_{4}\right)\right. \text {. } \\
& \left.\left.\cdot\left[2 v_{1}+\left(c_{I I}\left(c_{I I}-\sqrt{c_{I I}^{2}-4 b_{I I}}\right)-2 b_{I I}\right) v_{5}+\left(c_{I I}\left(c_{I I}+\sqrt{c_{I I}^{2}-4 b_{I I}}\right)-2 b_{I I}\right) v_{6}\right]+1\right]\right\}
\end{aligned}
$$

Então, de acordo com o Teorema da Variedade Central (Guckenheimer e Holmes, 1983; Wiggins, 1990; Monteiro, 2002), procura-se a variedade central $W^{C}$ que seja tangente ao respectivo subespaço central $E^{C}$ no ponto de equilíbrio $P_{3}$. Assim, para $y, z, \mu_{I}$ e $\mu_{I I}$ suficientemente pequenos, tem-se:

$$
\begin{aligned}
W^{C}(0)=\{ & \left(v_{1}, v_{2}, v_{3}, v_{4}, v_{5}, v_{6}, \mu_{I}, \mu_{I I}\right) \in \mathfrak{R}^{4} / v_{1}=y, v_{2}=z, \\
& v_{3}=h_{1}\left(y, z, \mu_{I}, \mu_{I I}\right), v_{4}=h_{2}\left(y, z, \mu_{I}, \mu_{I I}\right), \\
& v_{5}=h_{3}\left(y, z, \mu_{I}, \mu_{I I}\right), v_{6}=h_{4}\left(y, z, \mu_{I}, \mu_{I I}\right), \\
& \left.h_{j}(0,0,0,0)=0, D h_{j}(0,0,0,0)=0, j=1,2,3,4\right\}
\end{aligned}
$$


Considere-se então, os seguintes polinômios estimadores $h_{1}\left(y, z, \mu_{I}, \mu_{I I}\right)$, $h_{2}\left(y, z, \mu_{I}, \mu_{I I}\right), h_{3}\left(y, z, \mu_{I}, \mu_{I I}\right)$ e $h_{4}\left(y, z, \mu_{I}, \mu_{I I}\right)$ compostos por monômios do segundo grau:

$$
\begin{aligned}
& h_{1}\left(y, z, \mu_{I}, \mu_{I I}\right)=\alpha_{1} \cdot y^{2}+\alpha_{2} \cdot z^{2}+\alpha_{3} \cdot \mu_{I}^{2}+\alpha_{4} \cdot \mu_{I I}^{2}+\alpha_{5} \cdot y \cdot z+\alpha_{6} \cdot y \cdot \mu_{I}+ \\
& +\alpha_{7} \cdot y \cdot \mu_{I I}+\alpha_{8} \cdot z \cdot \mu_{I}+\alpha_{9} \cdot z \cdot \mu_{I I}+\alpha_{10} \cdot \mu_{I} \cdot \mu_{I I},\left(\alpha_{j} \in \mathbb{R}, j=1, \ldots, 10\right) \\
& h_{2}\left(y, z, \mu_{I}, \mu_{I I}\right)=\beta_{1} \cdot y^{2}+\beta_{2} \cdot z^{2}+\beta_{3} \cdot \mu_{I}^{2}+\beta_{4} \cdot \mu_{I I}^{2}+\beta_{5} \cdot y \cdot z+\beta_{6} \cdot y \cdot \mu_{I}+ \\
& +\beta_{7} \cdot y \cdot \mu_{I I}+\beta_{8} \cdot z \cdot \mu_{I}+\beta_{9} \cdot z \cdot \mu_{I I}+\beta_{10} \cdot \mu_{I} \cdot \mu_{I I},\left(\beta_{j} \in \mathbb{R}, j=1, \ldots, 10\right) \\
& h_{3}\left(y, z, \mu_{I}, \mu_{I I}\right)=\gamma_{1} \cdot y^{2}+\gamma_{2} \cdot z^{2}+\gamma_{3} \cdot \mu_{I}^{2}+\gamma_{4} \cdot \mu_{I I}^{2}+\gamma_{5} \cdot y \cdot z+\gamma_{6} \cdot y \cdot \mu_{I}+ \\
& +\gamma_{7} \cdot y \cdot \mu_{I I}+\gamma_{8} \cdot z \cdot \mu_{I}+\gamma_{9} \cdot z \cdot \mu_{I I}+\gamma_{10} \cdot \mu_{I} \cdot \mu_{I I},\left(\gamma_{j} \in \mathbb{R}, j=1, \ldots, 10\right) \\
& h_{4}\left(y, z, \mu_{I}, \mu_{I I}\right)=\theta_{1} \cdot y^{2}+\theta_{2} \cdot z^{2}+\theta_{3} \cdot \mu_{I}^{2}+\theta_{4} \cdot \mu_{I I}^{2}+\theta_{5} \cdot y \cdot z+\theta_{6} \cdot y \cdot \mu_{I}+ \\
& +\theta_{7} \cdot y \cdot \mu_{I I}+\theta_{8} \cdot z \cdot \mu_{I}+\theta_{9} \cdot z \cdot \mu_{I I}+\theta_{10} \cdot \mu_{I} \cdot \mu_{I I},\left(\theta_{j} \in \mathbb{R}, j=1, \ldots, 10\right)
\end{aligned}
$$

Pelo teorema de Carr (Carr, 1981; Guckenheimer e Holmes, 1983; Wiggins, 1990; Monteiro, 2002), a variedade central $W^{c}$ deve satisfazer $N\left(h\left(y, z, \mu_{I}, \mu_{I I}\right)\right)=0$. Portanto, tem-se:

$$
\begin{aligned}
& \frac{\partial h_{1}\left(y, z, \mu_{I}, \mu_{I I}\right)}{\partial y}\left[\lambda_{1} \cdot y+f_{1}\left(y, z, h_{1}, h_{2}, h_{3}, h_{4}, \mu_{I}, \mu_{I I}\right)\right]+ \\
& +\frac{\partial h_{1}\left(y, z, \mu_{I}, \mu_{I I}\right)}{\partial z}\left[\lambda_{2} \cdot z+f_{2}\left(y, z, h_{1}, h_{2}, h_{3}, h_{4}, \mu_{I}, \mu_{I I}\right)\right]- \\
& -\lambda_{3} \cdot h_{1}\left(y, z, \mu_{I}, \mu_{I I}\right)-f_{3}\left(y, z, h_{1}, h_{2}, h_{3}, h_{4}, \mu_{I}, \mu_{I I}\right)=0 \\
& \frac{\partial h_{2}\left(y, z, \mu_{I}, \mu_{I I}\right)}{\partial y}\left[\lambda_{1} \cdot y+f_{1}\left(y, z, h_{1}, h_{2}, h_{3}, h_{4}, \mu_{I}, \mu_{I I}\right)\right]+ \\
& +\frac{\partial h_{2}\left(y, z, \mu_{I}, \mu_{I I}\right)}{\partial z}\left[\lambda_{2} \cdot z+f_{2}\left(y, z, h_{1}, h_{2}, h_{3}, h_{4}, \mu_{I}, \mu_{I I}\right)\right]- \\
& -\lambda_{4} \cdot h_{2}\left(y, z, \mu_{I}, \mu_{I I}\right)-f_{4}\left(y, z, h_{1}, h_{2}, h_{3}, h_{4}, \mu_{I}, \mu_{I I}\right)=0 \\
& \frac{\partial h_{3}\left(y, z, \mu_{I}, \mu_{I I}\right)}{\partial y}\left[\lambda_{1} \cdot y+f_{1}\left(y, z, h_{1}, h_{2}, h_{3}, h_{4}, \mu_{I}, \mu_{I I}\right)\right]+ \\
& +\frac{\partial h_{3}\left(y, z, \mu_{I}, \mu_{I I}\right)}{\partial z}\left[\lambda_{2} \cdot z+f_{2}\left(y, z, h_{1}, h_{2}, h_{3}, h_{4}, \mu_{I}, \mu_{I I}\right)\right]- \\
& -\lambda_{5} \cdot h_{3}\left(y, z, \mu_{I}, \mu_{I I}\right)-f_{5}\left(y, z, h_{1}, h_{2}, h_{3}, h_{4}, \mu_{I}, \mu_{I I}\right)=0
\end{aligned}
$$




$$
\begin{aligned}
& \frac{\partial h_{4}\left(y, z, \mu_{I}, \mu_{I I}\right)}{\partial y}\left[\lambda_{1} \cdot y+f_{1}\left(y, z, h_{1}, h_{2}, h_{3}, h_{4}, \mu_{I}, \mu_{I I}\right)\right]+ \\
& +\frac{\partial h_{4}\left(y, z, \mu_{I}, \mu_{I I}\right)}{\partial z}\left[\lambda_{2} \cdot z+f_{2}\left(y, z, h_{1}, h_{2}, h_{3}, h_{4}, \mu_{I}, \mu_{I I}\right)\right]- \\
& \quad-\lambda_{6} \cdot h_{4}\left(y, z, \mu_{I}, \mu_{I I}\right)-f_{6}\left(y, z, h_{1}, h_{2}, h_{3}, h_{4}, \mu_{I}, \mu_{I I}\right)=0
\end{aligned}
$$

Utilizando (4.45) e (4.47) em (4.48) é possível obter, igualando-se os termos de igual potência, os coeficientes de $h_{1}\left(y, z, \mu_{I}, \mu_{I I}\right), h_{2}\left(y, z, \mu_{I}, \mu_{I I}\right), h_{3}\left(y, z, \mu_{I}, \mu_{I I}\right)$ e $h_{4}\left(y, z, \mu_{I}, \mu_{I I}\right):$

$$
\begin{aligned}
& y^{2}: \alpha_{1}=0, \beta_{1}=0, \gamma_{1}=0, \theta_{1}=0 \\
& z^{2}: \alpha_{2}=0, \beta_{2}=0, \gamma_{2}=0, \theta_{2}=0 \\
& \mu_{I}^{2}: \alpha_{3}=0, \beta_{3}=0, \gamma_{3}=0, \theta_{3}=0 \\
& \mu_{I I}^{2}: \alpha_{4}=0, \beta_{4}=0, \gamma_{4}=0, \theta_{4}=0 \\
& y \cdot z: \alpha_{5}=0, \beta_{5}=0, \gamma_{5}=0, \theta_{5}=0 \\
& y \cdot \mu_{I}: \alpha_{6}=0, \beta_{6}=0, \gamma_{6}=0, \theta_{6}=0 \\
& y \cdot \mu_{I I}: \alpha_{7}=0, \beta_{7}=0, \gamma_{7}=\frac{\sqrt{c_{I I}^{2}-4 b_{I I}}}{c_{I I}^{2}-4 b_{I I}}, \theta_{7}=\frac{\sqrt{c_{I I}^{2}-4 b_{I I}}}{4 b_{I I}-c_{I I}^{2}} \\
& z \cdot \mu_{I}: \alpha_{8}=-\frac{\sqrt{1-4 b_{I}}}{4 b_{I}-1}, \beta_{6}=\frac{\sqrt{1-4 b_{I}}}{4 b_{I}-1}, \gamma_{8}=0, \theta_{8}=0 \\
& \mu_{I} \cdot \mu_{I I}: \alpha_{10}=0, \beta_{10}=0, \gamma_{10}=0, \theta_{10}=0 \\
& \mu_{I I}: \alpha_{9}=0, \beta_{9}=0, \gamma_{9}=\frac{\sqrt{c_{I I}^{2}-4 b_{I I}}}{4 b_{I I}-c_{I I}^{2}}, \theta_{9}=\frac{\sqrt{c_{I I}^{2}-4 b_{I I}}}{c_{I I}^{2}-4 b_{I I}}
\end{aligned}
$$

Logo, os polinômios estimadores são: 


$$
\begin{aligned}
& h_{1}\left(y, z, \mu_{I}, \mu_{I I}\right)=-\frac{\sqrt{1-4 b_{I}}}{4 b_{I}-1} \cdot z \cdot \mu_{I} \\
& h_{2}\left(y, z, \mu_{I}, \mu_{I I}\right)=+\frac{\sqrt{1-4 b_{I}}}{4 b_{I}-1} \cdot z \cdot \mu_{I} \\
& h_{3}\left(y, z, \mu_{I}, \mu_{I I}\right)=\frac{\sqrt{c_{I I}^{2}-4 b_{I I}}}{c_{I I}^{2}-4 b_{I I}} \cdot y \cdot \mu_{I I}+\frac{\sqrt{c_{I I}^{2}-4 b_{I I}}}{4 b_{I I}-c_{I I}^{2}} \cdot z \cdot \mu_{I I} \\
& h_{4}\left(y, z, \mu_{I}, \mu_{I I}\right)=\frac{\sqrt{c_{I I}^{2}-4 b_{I I}}}{4 b_{I I}-c_{I I}^{2}} \cdot y \cdot \mu_{I I}+\frac{\sqrt{c_{I I}^{2}-4 b_{I I}}}{c_{I I}^{2}-4 b_{I I}} \cdot z \cdot \mu_{I I}
\end{aligned}
$$

Substituindo (4.50) no sistema (4.45), pode-se obter, desprezando os termos de ordem superior, o campo de vetores restrito à variedade central:

$$
\left\{\begin{array}{l}
\dot{y}=-\mu_{I I} \cdot y+\mu_{I I} \cdot z \\
\dot{z}=+\mu_{I} \cdot z \\
\dot{\mu}_{I}=0 \\
\dot{\mu}_{I I}=0
\end{array}\right.
$$

Para obter o ponto de equilíbrio de (5.18), basta fazer:

$$
\left\{\begin{array}{c}
\dot{y}=0 \Rightarrow-\mu_{I I} \cdot y^{*}+\mu_{I I} \cdot z^{*}=0 \\
\dot{z}=0 \Rightarrow+\mu_{I} \cdot z^{*}=0 \Rightarrow z^{*}=0
\end{array} \Rightarrow y^{*}=0 \Rightarrow E=\left(y^{*}, z^{*}\right)=(0,0)\right.
$$

A matriz Jacobiana de (5.18), calculada no ponto de equilíbrio $E=(0,0)$ é:

$$
J_{E}=\left[\begin{array}{cc}
-\mu_{I I} & +\mu_{I I} \\
0 & +\mu_{I}
\end{array}\right]
$$

Os autovalores de (4.53) são:

- $\lambda_{1}=+\mu_{I}$

- $\lambda_{2}=-\mu_{I I}$

De (4.54) se conclui que:

- Para $\mu_{I}>0$ e $\mu_{I I}>0$ o ponto de equilíbrio é instável.

- Para $\mu_{I}>0$ e $\mu_{I I}<0$ o ponto de equilíbrio é instável. 
- Para $\mu_{I}<0$ e $\mu_{I I}<0$ o ponto de equilíbrio é instável.

- Para $\mu_{I}<0$ e $\mu_{I I}>0$ o ponto de equilíbrio é assintoticamente estável.

No capítulo VI serão realizadas conclusões mais detalhadas a respeito das expressões obtidas em (5.51), que tratam da estabilidade dos pontos de (4.55).

\subsubsection{ANÁLISE DA ESTABILIDADE DE $P_{4}=(0,0,0,-\pi, 0,0)$ VIA TVC}

Para a aplicação do TVC, faz-se necessária a expansão em série de Taylor das funções seno e co-seno em torno de $x_{1}^{*}=0$ e $x_{4}^{*}=-\pi$ :

$$
\operatorname{sen} x_{1} \approx x_{1}+\ldots, \cos x_{1} \approx 1+\ldots, \operatorname{sen} x_{4} \approx-\left(x_{4}+\pi\right)+\ldots \text { e } \cos x_{4} \approx-1+\ldots
$$

E, portanto, a partir de (4.40), tem-se:

- $\cos \left(x_{4}-x_{1}\right)=\cos x_{4} \cos x_{1}+\operatorname{sen} x_{4} \operatorname{sen} x_{1}=-1-x_{1}\left(x_{4}+\pi\right)$

- $\operatorname{sen}\left(x_{4}-x_{1}\right)=\operatorname{sen}_{4} \cos x_{1}-\operatorname{sen} x_{1} \cos x_{4}=-\left(x_{4}+\pi\right)+x_{1}$

Entretanto, o TVC exige que o ponto de equilíbrio seja a origem. Seja então a seguinte mudança de coordenadas:

$$
\bar{x}_{1}=x_{1}, \bar{x}_{2}=x_{2}, \bar{x}_{3}=x_{3}, \bar{x}_{4}=x_{4}+\pi, \bar{x}_{5}=x_{5} \text { e } \bar{x}_{6}=x_{6}
$$

Utilizando as expressões de (4.57), reescreve-se o sistema (4.8), já transladado para a origem de acordo com (4.58):

$$
\begin{aligned}
& \left\{\begin{array}{l}
\dot{\bar{x}}_{1}=\bar{x}_{2} \\
\dot{\bar{x}}_{2}=\bar{x}_{3} \\
\dot{\bar{x}}_{3}=-\frac{c_{I}}{b_{I}} \bar{x}_{3}-\frac{1}{b_{I}} \bar{x}_{2}-\frac{\mu_{I} \cdot a_{I}}{b_{I}} \bar{x}_{2}(+1)-\frac{\mu_{I}}{b_{I}}\left(+\bar{x}_{1}\right) \\
\dot{\bar{x}}_{4}=\bar{x}_{5} \\
\dot{\bar{x}}_{5}=\bar{x}_{6} \\
\dot{\bar{x}}_{6}=-\frac{c_{I I}}{b_{I I}} \bar{x}_{6}-\frac{1}{b_{I I}} \bar{x}_{5}-\frac{\mu_{I I} \cdot a_{I I}}{b_{I I}}\left(\bar{x}_{5}-\bar{x}_{2}\right)\left(-1-\bar{x}_{1} \bar{x}_{4}\right)-\frac{\mu_{I I}}{b_{I I}}\left(-\bar{x}_{4}+\bar{x}_{1}\right)
\end{array}\right. \\
& \dot{\mu}_{I}=0 \mathrm{e} \dot{\mu}_{I I}=0
\end{aligned}
$$


O sistema (4.43) pode ser representado matricialmente como $\dot{\bar{X}}=J_{P_{1,2}} \cdot \bar{X}+\bar{F}$, $\dot{\mu}_{I}=0$ e $\dot{\mu}_{I I}=0$, ou seja:

$$
\begin{aligned}
& {\left[\begin{array}{c}
\dot{\bar{x}}_{1} \\
\dot{\bar{x}}_{2} \\
\dot{\bar{x}}_{3} \\
\dot{\bar{x}}_{4} \\
\dot{\bar{x}}_{5} \\
\dot{\bar{x}}_{6}
\end{array}\right]=\left[\begin{array}{cccccc}
0 & 1 & 0 & 0 & 0 & 0 \\
0 & 0 & 1 & 0 & 0 & 0 \\
0 & -\frac{1}{b_{I}} & -\frac{c_{I}}{b_{I}} & 0 & 0 & 0 \\
0 & 0 & 0 & 0 & 1 & 0 \\
0 & 0 & 0 & 0 & 0 & 1 \\
0 & 0 & 0 & 0 & -\frac{1}{b_{I I}} & -\frac{c_{I I}}{b_{I I}}
\end{array}\right] \cdot\left[\begin{array}{l}
\bar{x}_{1} \\
\bar{x}_{2} \\
\bar{x}_{3} \\
\bar{x}_{4} \\
\bar{x}_{5} \\
\bar{x}_{6}
\end{array}\right]+} \\
& +\left[\begin{array}{c}
-\frac{\mu_{I} \cdot a_{I}}{b_{I}} \bar{x}_{2}-\frac{\mu_{I}}{b_{I}} \bar{x}_{1} \\
0 \\
\dot{\mu}_{I}=0 \mathrm{e} \dot{\mu}_{I I}=0 \\
-\frac{\mu_{I I} \cdot a_{I I}}{b_{I I}}\left(\bar{x}_{5}-\bar{x}_{2}\right)\left(-1-\bar{x}_{4} \cdot \bar{x}_{1}\right)-\frac{\mu_{I I}}{b_{I I}}\left(-\bar{x}_{4}+\bar{x}_{1}\right)
\end{array}\right]
\end{aligned}
$$

Conforme requerido pelo TVC, o sistema (4.60) precisa ser reescrito na forma canônica de Jordan. Para isso, define-se um novo conjunto de variáveis de estado $V=\left(v_{1}, v_{2}, v_{3}, v_{4}, v_{5}, v_{6}\right)^{T}$ de modo que $\bar{X}=M \cdot V$, sendo $M$ uma base composta pelos autovetores de $J_{P_{1,2,3,4}}$ de (4.9). Dessa forma, é possível dizer que $V=\left[M^{-1} \cdot J_{P_{1,2}} \cdot M\right] \cdot V+M^{-1} \cdot \bar{F}$, ou seja:

$$
\left[\begin{array}{l}
\dot{v}_{1} \\
\dot{v}_{2} \\
\dot{v}_{3} \\
\dot{v}_{4} \\
\dot{v}_{5} \\
\dot{v}_{6}
\end{array}\right]=\left[\begin{array}{cccccc}
\lambda_{1} & 0 & 0 & 0 & 0 & 0 \\
0 & \lambda_{2} & 0 & 0 & 0 & 0 \\
0 & 0 & \lambda_{3} & 0 & 0 & 0 \\
0 & 0 & 0 & \lambda_{4} & 0 & 0 \\
0 & 0 & 0 & 0 & \lambda_{5} & 0 \\
0 & 0 & 0 & 0 & 0 & \lambda_{6}
\end{array}\right] \cdot\left[\begin{array}{c}
v_{1} \\
v_{2} \\
v_{3} \\
v_{4} \\
v_{5} \\
v_{6}
\end{array}\right]+\left[\begin{array}{c}
f_{1} \\
f_{2} \\
f_{3} \\
f_{4} \\
f_{5} \\
f_{6}
\end{array}\right]
$$




$$
\dot{\mu}_{I}=0 \text { e } \dot{\mu}_{I I}=0
$$

Em (4.45), tem-se que:

- $\lambda_{1}=0$

- $\lambda_{2}=0$

- $\lambda_{3}=-\frac{\left(c_{I}+\sqrt{c_{I}^{2}-4 b_{I}}\right)}{2 b_{I}}$

- $\lambda_{4}=\frac{\left(\sqrt{c_{I}^{2}-4 b_{I}}-c_{I}\right)}{2 b_{I}}$

- $\lambda_{5}=-\frac{\left(c_{I I}+\sqrt{c_{I I}^{2}-4 b_{I I}}\right)}{2 b_{I I}}$

- $\lambda_{6}=\frac{\left(\sqrt{c_{I I}^{2}-4 b_{I I}}-c_{I I}\right)}{2 b_{I I}}$

- $f_{1}=\frac{1}{2} \mu_{I I}\left\{2\left(v_{1}-v_{2}\right)+\left(2 b_{I}+\sqrt{1-4 b_{I}}-1\right) v_{3}-\left(-2 b_{I}+\sqrt{1-4 b_{I}}+1\right) v_{4}+\right.$

$+\left[c_{I I}\left(c_{I I}-\sqrt{c_{I I}^{2}-4 b_{I I}}\right)-2 b_{I I}\right] v_{5}+\left[c_{I I}\left(c_{I I}+\sqrt{c_{I I}^{2}-4 b_{I I}}\right)-2 b_{I I}\right] v_{6}-$

$-a_{I I}\left[-\left(\sqrt{1-4 b_{I}}-1\right) v_{3}+\left(\sqrt{1-4 b_{I}}+1\right) v_{4}-\right.$

$\left.-c_{I I} \cdot v_{5}+\sqrt{c_{I I}^{2}-4 b_{I I}} \cdot v_{5}-c_{I I} \cdot v_{6}-\sqrt{c_{I I}^{2}-4 b_{I I}} \cdot v_{6}\right]$.

$\cdot\left[-\frac{1}{4}\left(2 v_{2}-\left(2 b_{I}+\sqrt{1-4 b_{I}}-1\right) v_{3}+\left(-2 b_{I}+\sqrt{1-4 b_{I}}+1\right) v_{4}\right)\right.$.

$\left.\left.\cdot\left[2 v_{1}+\left(c_{I I}\left(c_{I I}-\sqrt{c_{I I}^{2}-4 b_{I I}}\right)-2 b_{I I}\right) v_{5}+\left(c_{I I}\left(c_{I I}+\sqrt{c_{I I}^{2}-4 b_{I I}}\right)-2 b_{I I}\right) v_{6}\right]-1\right]\right\}$

- $f_{2}=\frac{1}{2} \mu_{I}\left[-2 v_{2}-\left(\sqrt{1-4 b_{I}} \cdot a_{I}+a_{I}+2 b_{I}+\sqrt{1-4 b_{I}}-1\right) v_{3}+\right.$ $\left.+\left(\sqrt{1-4 b_{I}} \cdot a_{I}+a_{I}+2 b_{I}-\sqrt{1-4 b_{I}}-1\right) v_{4}\right]$

- $f_{3}=-\frac{1}{4 b_{I} \sqrt{1-4 b_{I}}}\left(\sqrt{1-4 b_{I}}+1\right) \mu_{I}\left\{2 v_{2}+\left[a_{I}\left(\sqrt{1-4 b_{I}}-1\right)-2 b_{I}-\right.\right.$ $\left.\left.-\sqrt{1-4 b_{I}}+1\right] v_{3}-\left(\sqrt{1-4 b_{I}} \cdot a_{I}+a_{I}+2 b_{I}-\sqrt{1-4 b_{I}}-1\right) v_{4}\right\}$ 
- $f_{4}=-\frac{1}{4 b_{I} \sqrt{1-4 b_{I}}}\left(\sqrt{1-4 b_{I}}+1\right) \mu_{I}\left\{2 v_{2}+\left[a_{I}\left(\sqrt{1-4 b_{I}}-1\right)-2 b_{I}-\right.\right.$

$$
\left.\left.-\sqrt{1-4 b_{I}}+1\right] v_{3}-\left(\sqrt{1-4 b_{I}} \cdot a_{I}+a_{I}+2 b_{I}-\sqrt{1-4 b_{I}}-1\right) v_{4}\right\}
$$

- $f_{5}=\frac{\mu_{I I}\left(c_{I I}+\sqrt{c_{I I}^{2}-4 b_{I I}}\right)}{4 b_{I I} \sqrt{c_{I I}^{2}-4 b_{I I}}}\left\{2\left(v_{1}-v_{2}\right)+\left(2 b_{I}+\sqrt{1-4 b_{I}}-1\right) v_{3}-\right.$

$$
-\left(-2 b_{I}+\sqrt{1-4 b_{I}}+1\right) v_{4}+\left[c_{I I}\left(c_{I I}-\sqrt{c_{I I}^{2}-4 b_{I I}}\right)-2 b_{I I}\right] v_{5}+
$$

$+\left[c_{I I}\left(c_{I I}+\sqrt{c_{I I}^{2}-4 b_{I I}}\right)-2 b_{I I}\right] v_{6}-a_{I I}\left[-\left(\sqrt{1-4 b_{I}}-1\right) v_{3}+\right.$

$\left.+\left(\sqrt{1-4 b_{I}}+1\right) v_{4}-c_{I I} \cdot v_{5}+\sqrt{c_{I I}^{2}-4 b_{I I}} \cdot v_{5}-c_{I I} \cdot v_{6}-\sqrt{c_{I I}^{2}-4 b_{I I}} \cdot v_{6}\right]$.

$\cdot\left[-\frac{1}{4}\left(2 v_{2}-\left(2 b_{I}+\sqrt{1-4 b_{I}}-1\right) v_{3}+\left(-2 b_{I}+\sqrt{1-4 b_{I}}+1\right) v_{4}\right)\right.$.

$\left.\left.\cdot\left[2 v_{1}+\left(c_{I I}\left(c_{I I}-\sqrt{c_{I I}^{2}-4 b_{I I}}\right)-2 b_{I I}\right) v_{5}+\left(c_{I I}\left(c_{I I}+\sqrt{c_{I I}^{2}-4 b_{I I}}\right)-2 b_{I I}\right) v_{6}\right]-1\right]\right\}$

- $f_{6}=-\frac{\mu_{I I}\left(c_{I I}-\sqrt{c_{I I}^{2}-4 b_{I I}}\right)}{4 b_{I I} \sqrt{c_{I I}^{2}-4 b_{I I}}}\left\{2\left(v_{1}-v_{2}\right)+\left(2 b_{I}+\sqrt{1-4 b_{I}}-1\right) v_{3}-\right.$

$-\left(-2 b_{I}+\sqrt{1-4 b_{I}}+1\right) v_{4}+\left[c_{I I}\left(c_{I I} \sqrt{c_{I I}^{2}-4 b_{I I}}\right)-2 b_{I I}\right] v_{5}+$

$+\left[c_{I I}\left(c_{I I}+\sqrt{c_{I I}^{2}-4 b_{I I}}\right)-2 b_{I I}\right] v_{6}-a_{I I}\left[-\left(\sqrt{1-4 b_{I}}-1\right) v_{3}+\right.$

$\left.+\left(\sqrt{1-4 b_{I}}+1\right) v_{4}-c_{I I} \cdot v_{5}+\sqrt{c_{I I}^{2}-4 b_{I I}} \cdot v_{5}-c_{I I} \cdot v_{6}-\sqrt{c_{I I}^{2}-4 b_{I I}} \cdot v_{6}\right]$.

$\cdot\left[-\frac{1}{4}\left(2 v_{2}-\left(2 b_{I}+\sqrt{1-4 b_{I}}-1\right) v_{3}+\left(-2 b_{I}+\sqrt{1-4 b_{I}}+1\right) v_{4}\right)\right.$.

$\left.\left.\cdot\left[2 v_{1}+\left(c_{I I}\left(c_{I I}-\sqrt{c_{I I}^{2}-4 b_{I I}}\right)-2 b_{I I}\right) v_{5}+\left(c_{I I}\left(c_{I I}+\sqrt{c_{I I}^{2}-4 b_{I I}}\right)-2 b_{I I}\right) v_{6}\right]-1\right]\right\}$

Então, de acordo com o Teorema da Variedade Central (Guckenheimer e Holmes, 1983; Wiggins, 1990; Monteiro, 2002), procura-se a variedade central $W^{C}$ que seja, tangente ao respectivo subespaço central $E^{C}$ no ponto de equilíbrio $P_{4}$. Assim, para $y, z, \mu_{I}$ e $\mu_{I I}$ suficientemente pequenos, tem-se: 


$$
\begin{aligned}
W_{1,2}^{C}(0)=\{ & \left(v_{1}, v_{2}, v_{3}, v_{4}, v_{5}, v_{6}, \mu_{I}, \mu_{I I}\right) \in \mathfrak{R}^{4} / v_{1}=y, v_{2}=z, \\
& v_{3}=h_{1}\left(y, z, \mu_{I}, \mu_{I I}\right), v_{4}=h_{2}\left(y, z, \mu_{I}, \mu_{I I}\right), \\
& v_{5}=h_{3}\left(y, z, \mu_{I}, \mu_{I I}\right), v_{6}=h_{4}\left(y, z, \mu_{I}, \mu_{I I}\right), \\
& \left.h_{j}(0,0,0,0)=0, D h_{j}(0,0,0,0)=0, j=1,2,3,4\right\}
\end{aligned}
$$

Considere-se então, os seguintes polinômios estimadores $h_{1}\left(y, z, \mu_{I}, \mu_{I I}\right)$, $h_{2}\left(y, z, \mu_{I}, \mu_{I I}\right), h_{3}\left(y, z, \mu_{I}, \mu_{I I}\right)$ e $h_{4}\left(y, z, \mu_{I}, \mu_{I I}\right)$ compostos por monômios do segundo grau:

$$
\begin{aligned}
& h_{1}\left(y, z, \mu_{I}, \mu_{I I}\right)=\alpha_{1} \cdot y^{2}+\alpha_{2} \cdot z^{2}+\alpha_{3} \cdot \mu_{I}^{2}+\alpha_{4} \cdot \mu_{I I}^{2}+\alpha_{5} \cdot y \cdot z+\alpha_{6} \cdot y \cdot \mu_{I}+ \\
& \quad+\alpha_{7} \cdot y \cdot \mu_{I I}+\alpha_{8} \cdot z \cdot \mu_{I}+\alpha_{9} \cdot z \cdot \mu_{I I}+\alpha_{10} \cdot \mu_{I} \cdot \mu_{I I},\left(\alpha_{j} \in \mathbb{R}, j=1, \ldots, 10\right) \\
& h_{2}\left(y, z, \mu_{I}, \mu_{I I}\right)=\beta_{1} \cdot y^{2}+\beta_{2} \cdot z^{2}+\beta_{3} \cdot \mu_{I}^{2}+\beta_{4} \cdot \mu_{I I}^{2}+\beta_{5} \cdot y \cdot z+\beta_{6} \cdot y \cdot \mu_{I}+ \\
& \quad+\beta_{7} \cdot y \cdot \mu_{I I}+\beta_{8} \cdot z \cdot \mu_{I}+\beta_{9} \cdot z \cdot \mu_{I I}+\beta_{10} \cdot \mu_{I} \cdot \mu_{I I},\left(\beta_{j} \in \mathbb{R}, j=1, \ldots, 10\right) \\
& h_{3}\left(y, z, \mu_{I}, \mu_{I I}\right)=\gamma_{1} \cdot y^{2}+\gamma_{2} \cdot z^{2}+\gamma_{3} \cdot \mu_{I}^{2}+\gamma_{4} \cdot \mu_{I I}^{2}+\gamma_{5} \cdot y \cdot z+\gamma_{6} \cdot y \cdot \mu_{I}+ \\
& \quad+\gamma_{7} \cdot y \cdot \mu_{I I}+\gamma_{8} \cdot z \cdot \mu_{I}+\gamma_{9} \cdot z \cdot \mu_{I I}+\gamma_{10} \cdot \mu_{I} \cdot \mu_{I I},\left(\gamma_{j} \in \mathbb{R}, j=1, \ldots, 10\right) \\
& h_{4}\left(y, z, \mu_{I}, \mu_{I I}\right)=\theta_{1} \cdot y^{2}+\theta_{2} \cdot z^{2}+\theta_{3} \cdot \mu_{I}^{2}+\theta_{4} \cdot \mu_{I I}^{2}+\theta_{5} \cdot y \cdot z+\theta_{6} \cdot y \cdot \mu_{I}+ \\
& \quad+\theta_{7} \cdot y \cdot \mu_{I I}+\theta_{8} \cdot z \cdot \mu_{I}+\theta_{9} \cdot z \cdot \mu_{I I}+\theta_{10} \cdot \mu_{I} \cdot \mu_{I I},\left(\theta_{j} \in \mathbb{R}, j=1, \ldots, 10\right)
\end{aligned}
$$

Pelo teorema de Carr (Carr, 1981; Guckenheimer e Holmes, 1983; Wiggins, 1990; Monteiro, 2002), a variedade central $W^{c}$ deve satisfazer $N\left(h\left(y, z, \mu_{I}, \mu_{I I}\right)\right)=0$. Portanto, tem-se:

$$
\begin{aligned}
& \frac{\partial h_{1}\left(y, z, \mu_{I}, \mu_{I I}\right)}{\partial y}\left[\lambda_{1} \cdot y+f_{1}\left(y, z, h_{1}, h_{2}, h_{3}, h_{4}, \mu_{I}, \mu_{I I}\right)\right]+ \\
& +\frac{\partial h_{1}\left(y, z, \mu_{I}, \mu_{I I}\right)}{\partial z}\left[\lambda_{2} \cdot z+f_{2}\left(y, z, h_{1}, h_{2}, h_{3}, h_{4}, \mu_{I}, \mu_{I I}\right)\right]- \\
& \quad-\lambda_{3} \cdot h_{1}\left(y, z, \mu_{I}, \mu_{I I}\right)-f_{3}\left(y, z, h_{1}, h_{2}, h_{3}, h_{4}, \mu_{I}, \mu_{I I}\right)=0 \\
& \begin{array}{l}
\frac{\partial h_{2}\left(y, z, \mu_{I}, \mu_{I I}\right)}{\partial y}\left[\lambda_{1} \cdot y+f_{1}\left(y, z, h_{1}, h_{2}, h_{3}, h_{4}, \mu_{I}, \mu_{I I}\right)\right]+ \\
+\frac{\partial h_{2}\left(y, z, \mu_{I}, \mu_{I I}\right)}{\partial z}\left[\lambda_{2} \cdot z+f_{2}\left(y, z, h_{1}, h_{2}, h_{3}, h_{4}, \mu_{I}, \mu_{I I}\right)\right]- \\
\quad-\lambda_{4} \cdot h_{2}\left(y, z, \mu_{I}, \mu_{I I}\right)-f_{4}\left(y, z, h_{1}, h_{2}, h_{3}, h_{4}, \mu_{I}, \mu_{I I}\right)=0
\end{array}
\end{aligned}
$$




$$
\begin{aligned}
& \frac{\partial h_{3}\left(y, z, \mu_{I}, \mu_{I I}\right)}{\partial y}\left[\lambda_{1} \cdot y+f_{1}\left(y, z, h_{1}, h_{2}, h_{3}, h_{4}, \mu_{I}, \mu_{I I}\right)\right]+ \\
& +\frac{\partial h_{3}\left(y, z, \mu_{I}, \mu_{I I}\right)}{\partial z}\left[\lambda_{2} \cdot z+f_{2}\left(y, z, h_{1}, h_{2}, h_{3}, h_{4}, \mu_{I}, \mu_{I I}\right)\right]- \\
& -\lambda_{5} \cdot h_{3}\left(y, z, \mu_{I}, \mu_{I I}\right)-f_{5}\left(y, z, h_{1}, h_{2}, h_{3}, h_{4}, \mu_{I}, \mu_{I I}\right)=0 \\
& \frac{\partial h_{4}\left(y, z, \mu_{I}, \mu_{I I}\right)}{\partial y}\left[\lambda_{1} \cdot y+f_{1}\left(y, z, h_{1}, h_{2}, h_{3}, h_{4}, \mu_{I}, \mu_{I I}\right)\right]+ \\
& +\frac{\partial h_{4}\left(y, z, \mu_{I}, \mu_{I I}\right)}{\partial z}\left[\lambda_{2} \cdot z+f_{2}\left(y, z, h_{1}, h_{2}, h_{3}, h_{4}, \mu_{I}, \mu_{I I}\right)\right]- \\
& -\lambda_{6} \cdot h_{4}\left(y, z, \mu_{I}, \mu_{I I}\right)-f_{6}\left(y, z, h_{1}, h_{2}, h_{3}, h_{4}, \mu_{I}, \mu_{I I}\right)=0
\end{aligned}
$$

Utilizando (4.61) e (4.63) em (4.48) é possível obter, igualando-se os termos de igual potência, os coeficientes de $h_{1}\left(y, z, \mu_{I}, \mu_{I I}\right), h_{2}\left(y, z, \mu_{I}, \mu_{I I}\right), h_{3}\left(y, z, \mu_{I}, \mu_{I I}\right)$ e $h_{4}\left(y, z, \mu_{I}, \mu_{I I}\right):$

$$
\begin{aligned}
& y^{2}: \alpha_{1}=0, \beta_{1}=0, \gamma_{1}=0, \theta_{1}=0 \\
& z^{2}: \alpha_{2}=0, \beta_{2}=0, \gamma_{2}=0, \theta_{2}=0 \\
& \mu_{I}^{2}: \alpha_{3}=0, \beta_{3}=0, \gamma_{3}=0, \theta_{3}=0 \\
& \mu_{I I}^{2}: \alpha_{4}=0, \beta_{4}=0, \gamma_{4}=0, \theta_{4}=0 \\
& y \cdot z: \alpha_{5}=0, \beta_{5}=0, \gamma_{5}=0, \theta_{5}=0 \\
& y \cdot \mu_{I}: \alpha_{6}=0, \beta_{6}=0, \gamma_{6}=0, \theta_{6}=0 \\
& y \cdot \mu_{I I}: \alpha_{7}=0, \beta_{7}=0, \gamma_{7}=\frac{\sqrt{c_{I I}^{2}-4 b_{I I}}}{c_{I I}^{2}-4 b_{I I}}, \theta_{7}=\frac{\sqrt{c_{I I}^{2}-4 b_{I I}}}{4 b_{I I}-c_{I I}^{2}} \\
& z \cdot \mu_{I}: \alpha_{8}=\frac{\sqrt{1-4 b_{I}}}{4 b_{I}-1}, \beta_{6}=-\frac{\sqrt{1-4 b_{I}}}{4 b_{I}-1}, \gamma_{8}=0, \theta_{8}=0
\end{aligned}
$$


$z \cdot \mu_{I I}: \alpha_{9}=0, \beta_{9}=0, \gamma_{9}=\frac{\sqrt{c_{I I}^{2}-4 b_{I I}}}{4 b_{I I}-c_{I I}^{2}}, \theta_{9}=\frac{\sqrt{c_{I I}^{2}-4 b_{I I}}}{c_{I I}^{2}-4 b_{I I}}$

$\mu_{I} \cdot \mu_{I I}: \alpha_{10}=0, \beta_{10}=0, \gamma_{10}=0, \theta_{10}=0$

Logo, os polinômios estimadores são:

$$
\begin{aligned}
& h_{1}\left(y, z, \mu_{I}, \mu_{I I}\right)=+\frac{\sqrt{1-4 b_{I}}}{4 b_{I}-1} \cdot z \cdot \mu_{I} \\
& h_{2}\left(y, z, \mu_{I}, \mu_{I I}\right)=-\frac{\sqrt{1-4 b_{I}}}{4 b_{I}-1} \cdot z \cdot \mu_{I} \\
& h_{3}\left(y, z, \mu_{I}, \mu_{I I}\right)=\frac{\sqrt{c_{I I}^{2}-4 b_{I I}}}{c_{I I}^{2}-4 b_{I I}} \cdot y \cdot \mu_{I I}+\frac{\sqrt{c_{I I}^{2}-4 b_{I I}}}{4 b_{I I}-c_{I I}^{2}} \cdot z \cdot \mu_{I I} \\
& h_{4}\left(y, z, \mu_{I}, \mu_{I I}\right)=\frac{\sqrt{c_{I I}^{2}-4 b_{I I}}}{4 b_{I I}-c_{I I}^{2}} \cdot y \cdot \mu_{I I}+\frac{\sqrt{c_{I I}^{2}-4 b_{I I}}}{c_{I I}^{2}-4 b_{I I}} \cdot z \cdot \mu_{I I}
\end{aligned}
$$

Substituindo (4.66) no sistema (4.61), pode-se obter, desprezando os termos de ordem superior, o campo de vetores restrito à variedade central:

$$
\left\{\begin{array}{l}
\dot{y}=+\mu_{I I} \cdot y-\mu_{I I} \cdot z \\
\dot{z}=-\mu_{I} \cdot z \\
\dot{\mu}_{I}=0 \\
\dot{\mu}_{I I}=0
\end{array}\right.
$$

Para obter o ponto de equilíbrio de (4.67), basta fazer:

$$
\left\{\begin{array}{l}
\dot{y}=0 \Rightarrow+\mu_{I I} \cdot y^{*}-\mu_{I I} \cdot z^{*}=0 \\
\dot{z}=0 \Rightarrow-\mu_{I} \cdot z^{*}=0 \Rightarrow z^{*}=0
\end{array} \Rightarrow y^{*}=0 \Rightarrow E=\left(y^{*}, z^{*}\right)=(0,0)\right.
$$

A matriz Jacobiana de (4.67), calculada no ponto de equilíbrio $E=(0,0)$ é:

$$
J_{E}=\left[\begin{array}{cc}
+\mu_{I I} & -\mu_{I I} \\
0 & -\mu_{I}
\end{array}\right]
$$

Os autovalores de (4.69) são:

- $\lambda_{1}=+\mu_{I}$ 
- $\lambda_{2}=-\mu_{I I}$

De (5.21) se conclui que:

- Para $\mu_{I}>0$ e $\mu_{I I}>0$ o ponto de equilíbrio é instável.

- Para $\mu_{I}>0$ e $\mu_{I I}<0$ o ponto de equilíbrio é instável.

- Para $\mu_{I}<0$ e $\mu_{I I}<0$ o ponto de equilíbrio é instável.

- Para $\mu_{I}<0$ e $\mu_{I I}>0$ o ponto de equilíbrio é assintoticamente estável.

No capítulo VI serão realizadas conclusões mais detalhadas a respeito das expressões obtidas em (5.21), que tratam da estabilidade dos pontos de (4.67).

No próximo capítulo, o estudo realizado no capítulo II é estendido para um PLL de uma rede OWMS Estrela - Cadeia Simples. 


\section{CAPÍTULO V}

\section{ESTUDO DA ESTABILIDADE DE UM NÓ ESCRAVO TIPO II FRENTE A UMA VARIAÇÃO DO TIPO RAMPA DE FASE NO MESTRE.}

No capítulo IV foi realizado o estudo da estabilidade de um nó escravo tipo II frente a uma variação do tipo degrau de fase no mestre. Neste capítulo, apresenta-se um estudo equivalente, porém com uma variação do tipo rampa de fase no mestre.

\subsection{OBTENÇÃO DOS PONTOS DE EQUILÍBRIO}

Conforme apresentado no capítulo 3 em (3.69), o sistema dinâmico que descreve um escravo II é da seguinte forma:

$$
\left\{\begin{array}{l}
b_{I} \cdot \dddot{\varphi}_{M, I}(t)+c_{I} \cdot \ddot{\varphi}_{M, I}(t)+\dot{\varphi}_{M, I}(t)+a_{I} \cdot \mu_{I} \cdot \dot{\varphi}_{M, I}(t) \cdot \cos \varphi_{M, I}(t)+ \\
+\mu_{I} \cdot \operatorname{sen} \varphi_{M, I}(t)=b_{I} \cdot \dddot{\theta}_{M}(t)+c_{I} \cdot \ddot{\theta}_{M}(t)+\dot{\theta}_{M}(t) \\
b_{I I} \dddot{\varphi}_{M, I I}(t)+c_{I I} \cdot \ddot{\varphi}_{M, I I}(t)+\dot{\varphi}_{M, I I}(t)+ \\
+a_{I I} \cdot \mu_{I I} \cdot\left(\dot{\varphi}_{M, I I}(t)-\dot{\varphi}_{M, I}(t)\right) \cdot \cos \left(\varphi_{M, I I}(t)-\varphi_{M, I}(t)\right)+ \\
\quad+\mu_{I I} \cdot \operatorname{sen}\left(\varphi_{M, I I}(t)-\varphi_{M, I}(t)\right)= \\
=b_{I I} \cdot \dddot{\theta}_{M}(t)+c_{I I} \cdot \ddot{\theta}_{M}(t)+\dot{\theta}_{M}(t)
\end{array}\right.
$$

Para variações $\theta_{M}(t)$ do tipo rampa de fase, pode-se escrever que:

$$
\dot{\theta}_{M}(t)=\Omega \Rightarrow \ddot{\theta}_{M}(t)=0 \Rightarrow \dddot{\theta}_{M}(t)=0
$$

Substituindo as entradas descritas em (5.2) no sistema dinâmico (5.1), tem-se:

$$
\left\{\begin{array}{c}
b_{I} \cdot \dddot{\varphi}_{M, I}(t)+c_{I} \cdot \ddot{\varphi}_{M, I}(t)+\dot{\varphi}_{M, I}(t)+a_{I} \cdot \mu_{I} \cdot \dot{\varphi}_{M, I}(t) \cdot \cos \varphi_{M, I}(t)+ \\
\quad+\mu_{I} \cdot \operatorname{sen} \varphi_{M, I}(t)=\Omega \\
b_{I I} \dddot{\varphi}_{M, I I}(t)+c_{I I} \cdot \ddot{\varphi}_{M, I I}(t)+\dot{\varphi}_{M, I I}(t)+ \\
+a_{I I} \cdot \mu_{I I} \cdot\left(\dot{\varphi}_{M, I I}(t)-\dot{\varphi}_{M, I}(t)\right) \cdot \cos \left(\varphi_{M, I I}(t)-\varphi_{M, I}(t)\right)+ \\
\quad+\mu_{I I} \cdot \operatorname{sen}\left(\varphi_{M, I I}(t)-\varphi_{M, I}(t)\right)=\Omega
\end{array}\right.
$$


Para a obtenção do sistema de equações no espaço de estados de (5.3) são definidas as seguintes variáveis de estado:

$$
\left\{\begin{array}{l}
x_{1}=\varphi_{M, I} \\
x_{2}=\dot{\varphi}_{M, I} \\
x_{3}=\ddot{\varphi}_{M, I} \\
x_{4}=\varphi_{M, I I} \\
x_{5}=\dot{\varphi}_{M, I I} \\
x_{6}=\ddot{\varphi}_{M, I I}
\end{array}\right.
$$

Assim, com $b_{I} \neq 0$ e $b_{I I} \neq 0$, se obtém o seguinte sistema de equações no espaço de estados:

$$
\left\{\begin{array}{l}
\dot{x}_{1}=x_{2} \\
\dot{x}_{2}=x_{3} \\
\dot{x}_{3}=\frac{\Omega}{b_{I}}-\frac{c_{I}}{b_{I}} x_{3}-\frac{1}{b_{I}} x_{2}-\frac{\mu_{I} \cdot a_{I}}{b_{I}} x_{2} \cos x_{1}-\frac{\mu_{I}}{b_{I}} \operatorname{sen} x_{1} \\
\dot{x}_{4}=x_{5} \\
\dot{x}_{5}=x_{6} \\
\dot{x}_{6}=\frac{\Omega}{b_{I I}}-\frac{c_{I I}}{b_{I I}} x_{6}-\frac{1}{b_{I I}} x_{5}-\frac{\mu_{I I} \cdot a_{I I}}{b_{I I}}\left(x_{5}-x_{2}\right) \cos \left(x_{4}-x_{1}\right)-\frac{\mu_{I I}}{b_{I I}} \operatorname{sen}\left(x_{4}-x_{1}\right)
\end{array}\right.
$$

Os pontos de equilíbrio de (5.5) podem ser obtidos como segue:

$$
\left\{\begin{array}{l}
\dot{x}_{1}=0 \Rightarrow x_{2}^{*}=0 \\
\dot{x}_{2}=0 \Rightarrow x_{3}^{*}=0 \\
\dot{x}_{3}=0 \Rightarrow \frac{\Omega}{b_{I}}-\frac{\mu_{I}}{b_{I}} \operatorname{sen} x_{1}^{*}=0 \Rightarrow \operatorname{sen} x_{1}^{*}=\frac{\Omega}{\mu_{I}}, \Omega \leq \mu_{I}, \mu_{I} \neq 0 \\
\dot{x}_{4}=0 \Rightarrow x_{5}^{*}=0 \\
\dot{x}_{5}=0 \Rightarrow x_{6}^{*}=0 \\
\dot{x}_{6}=0 \Rightarrow \frac{\Omega}{b_{I I}}-\frac{\mu_{I I}}{b_{I I}} \operatorname{sen}\left(x_{4}^{*}-x_{1}^{*}\right)=0 \Rightarrow \operatorname{sen}\left(x_{4}^{*}-x_{1}^{*}\right)=\frac{\Omega}{\mu_{I I}}, \Omega \leq \mu_{I I}, \mu_{I I} \neq 0
\end{array}\right.
$$

De (5.6) se conclui que:

- $\Omega=\mu_{I}$ e $\Omega=\mu_{I I} \Rightarrow x_{1}^{*}=+\frac{\pi}{2}$ e $\operatorname{sen}\left(x_{4}^{*}-\frac{\pi}{2}\right)=1 \Rightarrow$

$$
\Rightarrow-\cos x_{4}^{*}=1 \Rightarrow x_{4}^{*}=-\pi
$$


- $\Omega=\mu_{I}$ e $\Omega<\mu_{I I} \Rightarrow x_{1}^{*}=+\frac{\pi}{2}$ e $\operatorname{sen}\left(x_{4}^{*}-\frac{\pi}{2}\right)=\frac{\Omega}{\mu_{I I}} \Rightarrow$

$$
\begin{aligned}
& \Rightarrow x_{4}^{*}-\frac{\pi}{2}=\arccos \left[ \pm \sqrt{1-\left(\frac{\Omega}{\mu_{I}}\right)^{2}}\right] \\
& \therefore x_{4}^{*}=+\frac{\pi}{2}+\arccos \left[ \pm \sqrt{1-\left(\frac{\Omega}{\mu_{I}}\right)^{2}}\right]
\end{aligned}
$$

- $\Omega<\mu_{I}$ e $\Omega=\mu_{I I} \Rightarrow x_{1}^{*}=\arccos \left[ \pm \sqrt{1-\left(\frac{\Omega}{\mu_{I}}\right)^{2}}\right] \mathrm{e}$

$$
\begin{aligned}
& \operatorname{sen}\left(x_{4}^{*}-\arccos \left[ \pm \sqrt{1-\left(\frac{\Omega}{\mu_{I}}\right)^{2}}\right]\right)=1 \Rightarrow x_{4}^{*}-\arccos \left[ \pm \sqrt{1-\left(\frac{\Omega}{\mu_{I}}\right)^{2}}\right]=+\frac{\pi}{2} \\
& \therefore x_{4}^{*}=+\frac{\pi}{2}+\arccos \left[ \pm \sqrt{1-\left(\frac{\Omega}{\mu_{I}}\right)^{2}}\right]
\end{aligned}
$$

- $\Omega<\mu_{I}$ e $\Omega<\mu_{I I} \Rightarrow x_{1}^{*}=\arccos \left[ \pm \sqrt{1-\left(\frac{\Omega}{\mu_{I}}\right)^{2}}\right] \mathrm{e}$

$$
\begin{aligned}
& \operatorname{sen}\left(x_{4}^{*}-\arccos \left[ \pm \sqrt{1-\left(\frac{\Omega}{\mu_{I}}\right)^{2}}\right]\right)=\frac{\Omega}{\mu_{I I}} \Rightarrow \\
& \Rightarrow x_{4}^{*}-\arccos \left[ \pm \sqrt{1-\left(\frac{\Omega}{\mu_{I}}\right)^{2}}\right]=\arccos \left[ \pm \sqrt{1-\left(\frac{\Omega}{\mu_{I I}}\right)^{2}}\right] \Rightarrow \\
& \therefore x_{4}^{*}=\arccos \left[ \pm \sqrt{1-\left(\frac{\Omega}{\mu_{I}}\right)^{2}}\right]+\arccos \left[ \pm \sqrt{1-\left(\frac{\Omega}{\mu_{I I}}\right)^{2}}\right]
\end{aligned}
$$

Desse modo, há nove pontos de equilíbrio:

$$
\text { - } \Omega=\mu_{I}=\mu_{I I} \Rightarrow P=\left(+\frac{\pi}{2}, 0,0,-\pi, 0,0\right)
$$


- $\Omega=\mu_{I}<\mu_{I I} \Rightarrow P_{1,2}=\left(+\frac{\pi}{2}, 0,0,+\frac{\pi}{2}+\arccos \left[ \pm \sqrt{1-\left(\frac{\Omega}{\mu_{I I}}\right)^{2}}\right], 0,0\right)$

- $\Omega=\mu_{I I}<\mu_{I} \Rightarrow$

$$
\Rightarrow P_{3,4}=\left(\arccos \left[ \pm \sqrt{1-\left(\frac{\Omega}{\mu_{I}}\right)^{2}}\right], 0,0,+\frac{\pi}{2}+\arccos \left[ \pm \sqrt{1-\left(\frac{\Omega}{\mu_{I}}\right)^{2}}\right], 0,0\right)
$$

- $\Omega<\mu_{I}$ e $\Omega<\mu_{I I} \Rightarrow$

$$
\begin{aligned}
& \Rightarrow P_{5,6}=\left(\arccos \left[ \pm \sqrt{1-\left(\frac{\Omega}{\mu_{I}}\right)^{2}}\right], 0,0,\right. \\
& \left., \arccos \left[ \pm \sqrt{1-\left(\frac{\Omega}{\mu_{I}}\right)^{2}}\right]+\arccos \left[ \pm \sqrt{1-\left(\frac{\Omega}{\mu_{I I}}\right)^{2}}\right], 0,0\right) \\
& \Rightarrow P_{7,8}=\left[\arccos \left[ \pm \sqrt{1-\left(\frac{\Omega}{\mu_{I}}\right)^{2}}\right], 0,0,\right. \\
& \left., \arccos \left[ \pm \sqrt{1-\left(\frac{\Omega}{\mu_{I}}\right)^{2}}\right]+\arccos \left[\mp \sqrt{1-\left(\frac{\Omega}{\mu_{I I}}\right)^{2}}\right], 0,0\right)
\end{aligned}
$$

Como primeira iniciativa para o estudo qualitativo da estabilidade desses pontos procede-se à linearização do sistema dinâmico em torno dos mesmos, ou seja, calcula-se a matriz Jacobiana para cada um dos pontos de equilíbrio, verificando-se então, a parte real de seus autovalores. É o que será feito a partir da próxima seção.

5.2 ESTUDO DO PONTO $P=\left(+\frac{\pi}{2}, 0,0,-\pi, 0,0\right)$

\subsubsection{ANÁLISE LINEAR DA ESTABILIDADE}

A matriz Jacobiana de (5.5) calculada em $P$ é: 


$$
J_{P}=\left[\begin{array}{cccccc}
0 & 1 & 0 & 0 & 0 & 0 \\
0 & 0 & 1 & 0 & 0 & 0 \\
0 & -\frac{1}{b_{I}} & -\frac{c_{I}}{b_{I}} & 0 & 0 & 0 \\
0 & 0 & 0 & 0 & 1 & 0 \\
0 & 0 & 0 & 0 & 0 & 1 \\
0 & 0 & 0 & 0 & -\frac{1}{b_{I I}} & -\frac{c_{I I}}{b_{I I}}
\end{array}\right]
$$

Então, os autovalores de (5.9) são:

- $\quad \lambda_{1}=0$

- $\lambda_{2}=0$

- $\lambda_{3}=-\frac{\left(c_{I}+\sqrt{c_{I}^{2}-4 b_{I}}\right)}{2 b_{I}}$

- $\lambda_{4}=\frac{\left(\sqrt{c_{I}^{2}-4 b_{I}}-c_{I}\right)}{2 b_{I}}$

- $\lambda_{5}=-\frac{\left(c_{I I}+\sqrt{c_{I I}^{2}-4 b_{I I}}\right)}{2 b_{I I}}$

- $\lambda_{6}=\frac{\left(\sqrt{c_{I I}^{2}-4 b_{I I}}-c_{I I}\right)}{2 b_{I I}}$

Através da análise dos autovalores de (5.10), pode-se verificar que:

- Os autovalores $\lambda_{1}$ e $\lambda_{2}$ são nulos, independentemente dos valores ou relações que possam assumir os parâmetros envolvidos.

- Os autovalores $\lambda_{3}$ e $\lambda_{4}$ são ou reais com parte real negativa, para $c_{I}^{2} \geq 4 b_{I}$, ou pares de complexos conjugados com parte real negativa, para $c_{I}^{2}<4 b_{I}$.

- Os autovalores $\lambda_{5}$ e $\lambda_{6}$ são ou reais com parte real negativa, para $c_{I I}^{2} \geq 4 b_{I I}$, ou pares de complexos conjugados com parte real negativa, para $c_{I I}^{2}<4 b_{I I}$. 
Portanto, graças à existência de dois autovalores nulos, não é possível obter conclusões sobre a estabilidade de $P$ através da análise linear. Para investigar o comportamento do sistema no subespaço central, será aplicado o Teorema da Variedade Central (TVC).

\subsubsection{ANÁLISE VIA TVC}

Para a aplicação do TVC, faz-se necessária a expansão em série de Taylor das funções seno e co-seno em torno de $x_{1}^{*}=+\frac{\pi}{2}$ e $x_{4}^{*}=-\pi$ :

$$
\begin{aligned}
& \operatorname{sen} x_{1} \approx 1-\frac{1}{2}\left(x_{1}-\frac{\pi}{2}\right)^{2}+\ldots \text { e } \cos x_{1} \approx-\left(x_{1}+\frac{\pi}{2}\right)+\frac{1}{6}\left(x_{1}+\frac{\pi}{2}\right)^{3} \ldots, \\
& \operatorname{sen} x_{4} \approx-\left(x_{4}+\pi\right)+\frac{1}{6}\left(x_{4}+\pi\right)^{3} \ldots \text { e } \cos x_{4} \approx-1+\frac{1}{2}\left(x_{4}+\pi\right)^{2}+\ldots
\end{aligned}
$$

Entretanto, o TVC exige que o ponto de equilíbrio seja a origem. Portanto, considere-se a seguinte mudança de coordenadas:

$$
\bar{x}_{1}=x_{1}-\frac{\pi}{2}, \bar{x}_{2}=x_{2}, \bar{x}_{3}=x_{3}, \bar{x}_{4}=x_{4}+\pi, \bar{x}_{5}=x_{5} \text { e } \bar{x}_{6}=x_{6}
$$

A partir de (5.12) e (5.13), tem-se:

- $\cos \left(x_{4}-x_{1}\right)=\cos x_{4} \cos x_{1}+\operatorname{sen} x_{4} \operatorname{sen} x_{1}=$

$$
=\left[\frac{\bar{x}_{1}^{3}}{6}-\bar{x}_{1}\right] \cdot\left[\frac{\bar{x}_{4}^{2}}{2}-1\right]+\left[1-\frac{\bar{x}_{1}^{2}}{2}\right] \cdot\left[\frac{\bar{x}_{4}^{3}}{6}-\bar{x}_{4}\right]
$$

- $\operatorname{sen}\left(x_{4}-x_{1}\right)=\operatorname{sen} x_{4} \cos x_{1}-\operatorname{sen} x_{1} \cos x_{4}=$

$$
=\left[\frac{\bar{x}_{1}^{3}}{6}-\bar{x}_{1}\right] \cdot\left[\frac{\bar{x}_{4}^{3}}{6}-\bar{x}_{4}\right]-\left[1-\frac{\bar{x}_{1}^{2}}{2}\right] \cdot\left[\frac{\bar{x}_{4}^{2}}{2}-1\right]
$$

Utilizando as expressões de (5.14), reescreve-se o sistema (5.5), já transladado para a origem: 


$$
\left\{\begin{array}{l}
\dot{\bar{x}}_{1}=\bar{x}_{2} \\
\dot{\bar{x}}_{2}=\bar{x}_{3} \\
\dot{\bar{x}}_{3}=-\frac{c_{I}}{b_{I}} \bar{x}_{3}-\frac{1}{b_{I}} \bar{x}_{2}-\frac{\mu \cdot a_{I}}{b_{I}} \bar{x}_{2} \cdot \bar{x}_{1}^{3}+\frac{\mu}{2 b_{I}} \bar{x}_{1}^{2} \\
\dot{\bar{x}}_{4}=x_{5} \\
\dot{\bar{x}}_{5}=x_{6} \\
\dot{\bar{x}}_{6}=-\frac{c_{I I}}{b_{I I}} \bar{x}_{6}-\frac{1}{b_{I I}} \bar{x}_{5}-\frac{\mu \cdot a_{I I}}{b_{I I}}\left(\bar{x}_{5}-\bar{x}_{2}\right)\left\{\left[\frac{\bar{x}_{1}^{3}}{6}-\bar{x}_{1}\right] \cdot\left[\frac{\bar{x}_{4}^{2}}{2}-1\right]+\right. \\
\left.+\left[1-\frac{\bar{x}_{1}^{2}}{2}\right] \cdot\left[\frac{\bar{x}_{4}^{3}}{6}-\bar{x}_{4}\right]\right\}-\frac{\mu}{b_{I I}}\left\{\left[\frac{\bar{x}_{1}^{3}}{6}-\bar{x}_{1}\right] \cdot\left[\frac{\bar{x}_{4}^{3}}{6}-\bar{x}_{4}\right]-\left[1-\frac{\bar{x}_{1}^{2}}{2}\right] \cdot\left[\frac{\bar{x}_{4}^{2}}{2}-1\right]\right\}
\end{array}\right.
$$

Sendo que em (5.15), tem-se $\mu=\mu_{I}=\mu_{I I}=\Omega$.

O sistema (5.15) pode ser representado matricialmente como $\dot{\bar{X}}=J_{P} \cdot \bar{X}+\bar{F}$, ou seja:

$$
\left[\begin{array}{c}
\dot{\bar{x}}_{1} \\
\dot{\bar{x}}_{2} \\
\dot{\bar{x}}_{3} \\
\dot{\bar{x}}_{4} \\
\dot{\bar{x}}_{5} \\
\dot{\bar{x}}_{6}
\end{array}\right]=\left[\begin{array}{cccccc}
0 & 1 & 0 & 0 & 0 & 0 \\
0 & 0 & 1 & 0 & 0 & 0 \\
0 & -\frac{1}{b_{I}} & -\frac{c_{I}}{b_{I}} & 0 & 0 & 0 \\
0 & 0 & 0 & 0 & 1 & 0 \\
0 & 0 & 0 & 0 & 0 & 1 \\
0 & 0 & 0 & 0 & -\frac{1}{b_{I I}} & -\frac{c_{I I}}{b_{I I}}
\end{array}\right] \cdot\left[\begin{array}{c}
\bar{x}_{1} \\
\bar{x}_{2} \\
\bar{x}_{3} \\
\bar{x}_{4} \\
\bar{x}_{5} \\
\bar{x}_{6}
\end{array}\right]+
$$

$$
+\left[\begin{array}{c}
0 \\
0 \\
-\frac{\mu \cdot a_{I}}{b_{I}} \bar{x}_{2} \cdot \bar{x}_{1}^{3}+\frac{\mu}{2 b_{I}} \bar{x}_{1}^{2} \\
0 \\
0 \\
-\frac{\mu \cdot a_{I I}}{b_{I I}}\left(\bar{x}_{5}-\bar{x}_{2}\right)\left\{\left[\frac{\bar{x}_{1}^{3}}{6}-\bar{x}_{1}\right] \cdot\left[\frac{\bar{x}_{4}^{2}}{2}-1\right]+\left[1-\frac{\bar{x}_{1}^{2}}{2}\right] \cdot\left[\frac{\bar{x}_{4}^{3}}{6}-\bar{x}_{4}\right]\right\}- \\
-\frac{\mu}{b_{I I}}\left\{\left[\frac{\bar{x}_{1}^{3}}{6}-\bar{x}_{1}\right] \cdot\left[\frac{\bar{x}_{4}^{3}}{6}-\bar{x}_{4}\right]-\left[1-\frac{\bar{x}_{1}^{2}}{2}\right] \cdot\left[\frac{\bar{x}_{4}^{2}}{2}-1\right]\right\}
\end{array}\right]
$$


Conforme requerido pelo TVC, o sistema (5.16) precisa ser reescrito na forma canônica de Jordan. Para isso, define-se um novo conjunto de variáveis de estado $V=\left(v_{1}, v_{2}, v_{3}, v_{4}, v_{5}, v_{6}\right)^{T}$ de modo que $\bar{X}=M \cdot V$, sendo $M$ uma base composta pelos autovetores de $J_{P}$ de (5.10). Assim, é possível se estabelecer a seguinte relação entre as variáveis de estado $X$ e $V$ :

$$
\begin{aligned}
& \bar{x}_{1}=v_{2}+\frac{1}{2}\left(c_{I} \cdot \delta_{I}-2 b_{I}\right) v_{3}+\frac{1}{2}\left(c_{I} \cdot \varepsilon_{I}-2 b_{I}\right) v_{4} \\
& \bar{x}_{2}=\frac{1}{2} \delta_{I} \cdot v_{3}-\frac{1}{2} \varepsilon_{I} \cdot v_{4} \\
& \bar{x}_{3}=v_{3}+v_{4} \\
& \bar{x}_{4}=v_{1}+\frac{1}{2}\left(c_{I I} \cdot \delta_{I I}-2 b_{I I}\right) v_{5}+\frac{1}{2}\left(c_{I I} \cdot \varepsilon_{I I}-2 b_{I I}\right) v_{6} \\
& \bar{x}_{5}=\frac{1}{2} \delta_{I I} \cdot v_{5}-\frac{1}{2} \varepsilon_{I I} \cdot v_{6} \\
& \bar{x}_{6}=v_{5}+v_{6}
\end{aligned}
$$

Sendo que em (5.17), tem-se: $\delta=c-\sqrt{c^{2}-4 b}$ e $\varepsilon=c+\sqrt{c^{2}-4 b}$.

Dessa forma, é possível fazer $V=\left[M^{-1} \cdot J_{P} \cdot M\right] \cdot V+M^{-1} \cdot \bar{F}$, ou seja:

$$
\left[\begin{array}{l}
\dot{v}_{1} \\
\dot{v}_{2} \\
\dot{v}_{3} \\
\dot{v}_{4} \\
\dot{v}_{5} \\
\dot{v}_{6}
\end{array}\right]=\left[\begin{array}{cccccc}
\lambda_{1} & 0 & 0 & 0 & 0 & 0 \\
0 & \lambda_{2} & 0 & 0 & 0 & 0 \\
0 & 0 & \lambda_{3} & 0 & 0 & 0 \\
0 & 0 & 0 & \lambda_{4} & 0 & 0 \\
0 & 0 & 0 & 0 & \lambda_{5} & 0 \\
0 & 0 & 0 & 0 & 0 & \lambda_{6}
\end{array}\right] \cdot\left[\begin{array}{l}
v_{1} \\
v_{2} \\
v_{3} \\
v_{4} \\
v_{5} \\
v_{6}
\end{array}\right]+\left[\begin{array}{l}
f_{1} \\
f_{2} \\
f_{3} \\
f_{4} \\
f_{5} \\
f_{6}
\end{array}\right]
$$

Em (5.17), tem-se que:

- $\lambda_{1}=0$

- $\lambda_{2}=0$ 
- $\lambda_{3}=-\frac{\left(c_{I}+\sqrt{c_{I}^{2}-4 b_{I}}\right)}{2 b_{I}}$

- $\lambda_{4}=\frac{\left(\sqrt{c_{I}^{2}-4 b_{I}}-c_{I}\right)}{2 b_{I}}$

- $\lambda_{5}=-\frac{\left(c_{I I}+\sqrt{c_{I I}^{2}-4 b_{I I}}\right)}{2 b_{I I}}$

- $\lambda_{6}=\frac{\left(\sqrt{c_{I I}^{2}-4 b_{I I}}-c_{I I}\right)}{2 b_{I I}}$

- $\left[\begin{array}{l}f_{1} \\ f_{2} \\ f_{3} \\ f_{4} \\ f_{5} \\ f_{6}\end{array}\right]=M^{-1} \cdot \bar{F}$

Por questão de simplicidade, optou-se por omitir os termos não-lineares $f_{1}$, $f_{2}, f_{3}, f_{4}, f_{5}$ e $f_{6}$ devido a sua extensão.

Então, de acordo com o Teorema da Variedade Central (Guckenheimer e Holmes, 1983; Wiggins, 1990; Monteiro, 2002), procura-se a variedade central $W^{C}$ que seja tangente subespaço central $E^{C}$ no ponto de equilíbrio $P$. Assim, para $y$ e $z$ suficientemente pequenos, tem-se:

$$
\begin{aligned}
W^{c}(0)=\{ & \left(v_{1}, v_{2}, v_{3}, v_{4}, v_{5}, v_{6}\right) \in \mathfrak{R}^{4} / v_{1}=y, v_{2}=z, \\
& v_{3}=h_{1}(y, z), v_{4}=h_{2}(y, z), \\
& v_{5}=h_{3}(y, z), v_{6}=h_{4}(y, z), \\
& \left.h_{j}(0,0)=0, D h_{j}(0,0)=0, j=1,2,3,4\right\}
\end{aligned}
$$

Considere-se então, os seguintes polinômios estimadores do terceiro grau $h_{1}(y, z), h_{2}(y, z), h_{3}(y, z)$ e $h_{4}(y, z)$ :

$$
\begin{aligned}
& h_{1}(y, z)=\alpha_{1} \cdot y^{3}+\alpha_{2} \cdot z^{3}+\alpha_{3} \cdot y^{2} \cdot z+\alpha_{4} \cdot y \cdot z^{2}+\alpha_{5} \cdot y^{2}+\alpha_{6} \cdot z^{2}+ \\
& \quad+\alpha_{7} \cdot y \cdot z,\left(\alpha_{j} \in \mathbb{R}, j=1, \ldots, 7\right)
\end{aligned}
$$




$$
\begin{aligned}
& \begin{array}{l}
h_{2}(y, z)=\beta_{1} \cdot y^{3}+\beta_{2} \cdot z^{3}+\beta_{3} \cdot y^{2} \cdot z+\beta_{4} \cdot y \cdot z^{2}+\beta_{5} \cdot y^{2}+\beta_{6} \cdot z^{2}+ \\
\quad+\beta_{7} \cdot y \cdot z,\left(\beta_{j} \in \mathbb{R}, j=1, \ldots, 7\right)
\end{array} \\
& \begin{array}{l}
h_{3}(y, z)=\gamma_{1} \cdot y^{3}+\gamma_{2} \cdot z^{3}+\gamma_{3} \cdot y^{2} \cdot z+\gamma_{4} \cdot y \cdot z^{2}+\gamma_{5} \cdot y^{2}+\gamma_{6} \cdot z^{2}+ \\
\quad+\gamma_{7} \cdot y \cdot z,\left(\gamma_{j} \in \mathbb{R}, j=1, \ldots, 7\right)
\end{array} \\
& \quad \begin{array}{l}
h_{4}(y, z)=\theta_{1} \cdot y^{3}+\theta_{2} \cdot z^{3}+\theta_{3} \cdot y^{2} \cdot z+\theta_{4} \cdot y \cdot z^{2}+\theta_{5} \cdot y^{2}+\theta_{6} \cdot z^{2}+ \\
\quad+\theta_{7} \cdot y \cdot z,\left(\theta_{j} \in \mathbb{R}, j=1, \ldots, 7\right)
\end{array}
\end{aligned}
$$

Pelo teorema de Carr (Carr, 1981; Guckenheimer e Holmes, 1983; Wiggins, 1990), a variedade central $W^{c}$ deve satisfazer $N(h(y, z))=0$. Portanto, tem-se:

$$
\begin{aligned}
& \frac{\partial h_{1}(y, z)}{\partial y}\left[\lambda_{1} \cdot x+f_{1}\left(y, z, h_{1}, h_{2}, h_{3}, h_{4}\right)\right]+ \\
& +\frac{\partial h_{1}(y, z)}{\partial z}\left[\lambda_{2} \cdot x+f_{2}\left(y, z, h_{1}, h_{2}, h_{3}, h_{4}\right)\right]- \\
& \quad-\lambda_{3} \cdot h_{1}(y, z)-f_{3}\left(y, z, h_{1}, h_{2}, h_{3}, h_{4}\right)=0 \\
& \frac{\partial h_{2}(y, z)}{\partial z \quad}\left[\lambda_{2} \cdot x+f_{2}\left(y, z, h_{1}, h_{2}, h_{3}, h_{4}\right)\right]+ \\
& +\frac{\partial h_{2}(y, z)}{\partial z}\left[\lambda_{2} \cdot x+f_{2}\left(y, z, h_{1}, h_{2}, h_{3}, h_{4}\right)\right]- \\
& \quad-\lambda_{4} \cdot h_{2}(y, z)-f_{4}\left(y, z, h_{1}, h_{2}, h_{3}, h_{4}\right)=0 \\
& \frac{\partial h_{3}(y, z)}{\partial y \quad}\left[\lambda_{1} \cdot x+f_{1}\left(y, z, h_{1}, h_{2}, h_{3}, h_{4}\right)\right]+ \\
& +\frac{\partial h_{3}(y, z)}{\partial z}\left[\lambda \cdot x+f_{2}\left(y, z, h_{1}, h_{2}, h_{3}, h_{4}\right)\right]- \\
& -\lambda_{4} \cdot h_{5}(y, z)-f_{4}\left(y, z, h_{1}, h_{2}, h_{3}, h_{4}\right)=0 \\
& \quad-\lambda_{6} \cdot h_{4}(y, z)-f_{6}\left(y, z, h_{1}, h_{2}, h_{3}, h_{4}\right)=0 \\
& \frac{\partial h_{4}(y, z)}{\partial z}\left[\lambda_{2} \cdot x+f_{2}\left(y, z, h_{1}, h_{2}, h_{3}, h_{4}\right)\right] \\
& +\frac{\partial h_{4}(y, z)}{\partial z}\left[\lambda_{2} \cdot x+f_{2}\left(y, z, h_{1}, h_{2}, h_{3}, h_{4}\right)\right]-
\end{aligned}
$$

Utilizando (5.17) e (5.19) em (5.20) e resolvendo o sistema de equações, é possível obter, igualando-se a zero os termos de igual potência, os coeficientes de $h_{1}(y, z), h_{2}(y, z), h_{3}(y, z)$ e $h_{4}(y, z)$ : 


$$
\begin{aligned}
& y^{3}: \alpha_{1}=0, \beta_{1}=0, \\
& \gamma_{1}=\frac{\left(-c_{I I}^{2}+3 c_{I I} \sqrt{c_{I I}^{2}-4 b_{I I}}+4 b_{I I}-2 a_{I I} \sqrt{c_{I I}^{2}-4 b_{I I}}\right)}{-4 c_{I I}^{2}+16 b_{I I}} \mu^{2}, \\
& \theta_{1}=\frac{\left(-c_{I I}^{2}-3 c_{I I} \sqrt{c_{I I}^{2}-4 b_{I I}}+4 b_{I I}+2 a_{I I} \sqrt{c_{I I}^{2}-4 b_{I I}}\right)}{-4 c_{I I}^{2}+16 b_{I I}} \mu^{2} \\
& z^{3}: \alpha_{2}=\frac{\left(-c_{I}^{2}+3 c_{I} \sqrt{c_{I}^{2}-4 b_{I}}+4 b_{I}-2 a_{I} \sqrt{c_{I}^{2}-4 b_{I}}\right)}{-4 c_{I}^{2}+16 b_{I}} \mu^{2}, \\
& \beta_{2}=\frac{\left[2 a_{I} \sqrt{c_{I}^{2}-4 b_{I}}+4 b_{I}-c_{I}\left(c_{I}+3 \sqrt{c_{I}^{2}-4 b_{I}}\right)\right]}{-4 c_{I}^{2}+16 b_{I}} \mu^{2}, \\
& \gamma_{2}=-\frac{\left[\left(c_{I}-c_{I I}\right) \sqrt{c_{I I}^{2}-4 b_{I I}}\right]}{2 c_{I I}^{2}-8 b_{I I}} \mu^{2}, \theta_{2}=-\frac{\left[\left(c_{I I}-c_{I}\right) \sqrt{c_{I I}^{2}-4 b_{I I}}\right]}{2 c_{I I}^{2}-8 b_{I I}} \mu^{2}
\end{aligned}
$$

$$
\begin{aligned}
& y^{2}: \alpha_{3}=0, \beta_{3}=0, \\
& \gamma_{3}=-\frac{3\left(-c_{I I}^{2}+3 c_{I I} \sqrt{c_{I I}^{2}-4 b_{I I}}+4 b_{I I}-2 a_{I I} \sqrt{c_{I I}^{2}-4 b_{I I}}\right)}{-4 c_{I I}^{2}+16 b_{I I}} \mu^{2}, \\
& \theta_{3}=-\frac{3\left(-c_{I I}^{2}-3 c_{I I} \sqrt{c_{I I}^{2}-4 b_{I I}}+4 b_{I I}+2 a_{I I} \sqrt{c_{I I}^{2}-4 b_{I I}}\right)}{-4 c_{I I}^{2}+16 b_{I I}} \mu^{2}
\end{aligned}
$$

$$
\begin{aligned}
& z^{2}: \alpha_{4}=0, \beta_{4}=0, \\
& \gamma_{4}=-\frac{\left(-c_{I I}^{2}+4 c_{I I} \sqrt{c_{I I}^{2}-4 b_{I I}}+4 b_{I I}-\left(2 a_{I I}+c_{I}\right) \sqrt{c_{I I}^{2}-4 b_{I I}}\right)}{2 c_{I I}^{2}-8 b_{I I}} \mu^{2}, \\
& \theta_{4}=-\frac{\left(-c_{I I}^{2}-4 c_{I I} \sqrt{c_{I I}^{2}-4 b_{I I}}+4 b_{I I}+\left(2 a_{I I}+c_{I}\right) \sqrt{c_{I I}^{2}-4 b_{I I}}\right)}{2 c_{I I}^{2}-8 b_{I I}} \mu^{2}
\end{aligned}
$$




$$
\begin{aligned}
& y^{2} \cdot z: \alpha_{5}=0, \beta_{5}=0, \gamma_{5}=+\frac{\sqrt{c_{I I}^{2}-4 b_{I I}}}{2 c_{I I}^{2}-8 b_{I I}} \mu, \theta_{5}=-\frac{\sqrt{c_{I I}^{2}-4 b_{I I}}}{2 c_{I}^{2}-8 b_{I}} \mu \\
& y \cdot z^{2}: \alpha_{6}=-\frac{\sqrt{c_{I}^{2}-4 b_{I}}}{2 c_{I}^{2}-8 b_{I}} \mu, \beta_{6}=\frac{\sqrt{c_{I}^{2}-4 b_{I}}}{2 c_{I}^{2}-8 b_{I}} \mu, \gamma_{6}=-\frac{\sqrt{c_{I I}^{2}-4 b_{I I}}}{2 c_{I I}^{2}-8 b_{I I}} \mu, \\
& \theta_{6}=-\frac{\sqrt{c_{I I}^{2}-4 b_{I I}}}{2 c_{I}^{2}-8 b_{I}} \mu \\
& y \cdot z: \alpha_{7}=0, \beta_{7}=0, \gamma_{7}=-\frac{\sqrt{c_{I I}^{2}-4 b_{I I}}}{c_{I I}^{2}-4 b_{I I}} \mu, \theta_{7}=+\frac{\sqrt{c_{I I}^{2}-4 b_{I I}}}{c_{I I}^{2}-4 b_{I I}} \mu
\end{aligned}
$$

Com os coeficientes (5.21) dos polinômios de (5.19), pode-se obter, através do sistema (5.17), o campo de vetores restrito à variedade central, desprezando-se os termos de ordem superior:

$$
\left\{\begin{array}{l}
\dot{y}=\frac{\mu}{2}(y-z)\left\{\left(a_{I I}-c_{I I}\right) \mu \cdot y^{2}+2\left(c_{I I}-a_{I I}\right) z \cdot \mu \cdot y+y+z\left[\left(c_{I}-c_{I I}\right) z \cdot \mu-1\right]\right\} \\
\dot{z}=\frac{\mu}{2} z^{2}\left[\left(a_{I}-c_{I}\right) z \cdot \mu+1\right]
\end{array}\right.
$$

Para obter os pontos de equilíbrio de (5.22), basta fazer:

$$
\left\{\begin{array}{l}
\dot{y}=0 \\
\dot{z}=0
\end{array}\right.
$$

De (5.19) se obtém os seguintes possíveis valores para y e $z$ :

- $y=0$ e $z=0$

- $y=-\frac{1}{\left(a_{I}-c_{I}\right) \mu}$ e $z=-\frac{1}{\left(a_{I}-c_{I}\right) \mu},\left(a_{I}-c_{I}\right) \mu \neq 0$

- $y=-\frac{1}{\left(a_{I I}-c_{I I}\right) \mu}$ e $z=0,\left(a_{I I}-c_{I I}\right) \mu \neq 0$ 


$$
\begin{aligned}
& y=-\frac{-a_{I}-2 a_{I I}+c_{I}+2 c_{I I}+\sqrt{a_{I}^{2}-2 c_{I} \cdot a_{I}+4 a_{I I}^{2}-4 a_{I I}\left(c_{I}+c_{I I}\right)+c_{I}\left(c_{I}+4 c_{I I}\right)}}{2\left(a_{I}-c_{I}\right)\left(a_{I I}-c_{I I}\right) \mu} \\
& \mathrm{e} z=-\frac{1}{\left(a_{I}-c_{I}\right) \mu},\left(a_{I}-c_{I}\right)\left(a_{I I}-c_{I I}\right) \mu \neq 0 \\
& \text { - } y=-\frac{a_{I}+2 a_{I I}-c_{I}-2 c_{I I}+\sqrt{a_{I}^{2}-2 c_{I} \cdot a_{I}+4 a_{I I}^{2}-4 a_{I I}\left(c_{I}+c_{I I}\right)+c_{I}\left(c_{I}+4 c_{I I}\right)}}{2\left(a_{I}-c_{I}\right)\left(a_{I I}-c_{I I}\right) \mu} \\
& \mathrm{e} z=-\frac{1}{\left(a_{I}-c_{I}\right) \mu},\left(a_{I}-c_{I}\right)\left(a_{I I}-c_{I I}\right) \mu \neq 0
\end{aligned}
$$

Para o estudo das estabilidades dos pontos de equilíbrio de (5.20), é preciso obter a matriz Jacobiana de (5.19):

$$
J_{P}=\left[\begin{array}{ll}
\left.\frac{\partial \dot{y}}{\partial y}\right|_{P} & \left.\frac{\partial \dot{y}}{\partial z}\right|_{P} \\
\left.\frac{\partial \dot{z}}{\partial y}\right|_{P} & \left.\frac{\partial \dot{z}}{\partial z}\right|_{P}
\end{array}\right]
$$

Sendo que em (5.21), tem-se:

$$
\begin{aligned}
\frac{\partial \dot{y}}{\partial y}= & \frac{1}{2} \mu\left\{3\left(a_{I I}-c_{I I}\right) \mu \cdot y^{2}+\left[6\left(c_{I I}-a_{I I}\right) z \cdot \mu+2\right] y+\right. \\
& \left.+z\left[\left(2 a_{I I}-c_{I}-3 c_{I I}\right) z \cdot \mu-2\right]\right\} \\
\frac{\partial \dot{y}}{\partial z}= & \frac{1}{2} \mu\left\{3\left(c_{I I}-a_{I I}\right) \mu \cdot y^{2}+z\left[3\left(c_{I I}-c_{I}\right) z \cdot \mu+2\right] y+\right. \\
& \left.+2\left[\left(2 a_{I I}+c_{I}-3 c_{I I}\right) z \cdot \mu-1\right]\right\} \\
\frac{\partial \dot{z}}{\partial y}= & 0 \\
\frac{\partial \dot{z}}{\partial z}= & \frac{1}{2} z \cdot \mu\left[3\left(a_{I}-c_{I}\right) z \cdot \mu+2\right]
\end{aligned}
$$

Em seguida, verifica-se a parte real dos autovalores para a matriz Jacobiana (5.21) calculada em cada ponto de equilíbrio:

- $y=0$ e $z=0$

$$
\lambda_{1}=0 \text { e } \lambda_{2}=0
$$


- $y=-\frac{1}{\left(a_{I}-c_{I}\right) \mu}$ e $z=-\frac{1}{\left(a_{I}-c_{I}\right) \mu}$

$\lambda_{1}=\frac{1}{2\left(a_{I}-c_{I}\right)}, \lambda_{2}=\frac{c_{I}-a_{I I}}{2\left(a_{I}-c_{I}\right)^{2}}$

$\left(\operatorname{Re}\left(\lambda_{1}\right)<0 \wedge \operatorname{Re}\left(\lambda_{2}\right)<0\right) \Leftrightarrow\left(a_{I I}>a_{I} \wedge a_{I}<c_{I}<a_{I I}\right)$

$\left(\operatorname{Re}\left(\lambda_{1}\right)>0 \vee \operatorname{Re}\left(\lambda_{2}\right)>0\right) \Leftrightarrow$

$\left(a_{I I} \leq a_{I} \wedge c_{I} \neq a_{I}\right) \vee\left[a_{I I}>a_{I} \wedge\left(c_{I}<a_{I} \vee c_{I}>a_{I I}\right)\right]$

- $y=-\frac{1}{\left(a_{I I}-c_{I I}\right) \mu}$ e $z=0$

$\lambda_{1}=0$ e $\lambda_{2}=\frac{1}{2\left(a_{I I}-c_{I I}\right)}$

$\left(\operatorname{Re}\left(\lambda_{2}\right)>0\right) \Leftrightarrow\left(a_{I I}>c_{I I}\right)$

- $y=-\frac{-a_{I}-2 a_{I I}+c_{I}+2 c_{I I}+\sqrt{a_{I}^{2}-2 c_{I} \cdot a_{I}+4 a_{I I}^{2}-4 a_{I I}\left(c_{I}+c_{I I}\right)+c_{I}\left(c_{I}+4 c_{I I}\right)}}{2\left(a_{I}-c_{I}\right)\left(a_{I I}-c_{I I}\right) \mu}$

$\mathrm{e} z=-\frac{1}{\left(a_{I}-c_{I}\right) \mu}$

$\lambda_{1}=\frac{1}{2\left(a_{I}-c_{I}\right)}$,

$\lambda_{2}=\frac{4\left(a_{I I}-c_{I}\right)+\frac{\left(c_{I}-a_{I}\right) \sqrt{a_{I}^{2}-2 c_{I} \cdot a_{I}+4 a_{I I}^{2}-4 a_{I I}\left(c_{I}+c_{I I}\right)+c_{I}\left(c_{I}+4 c_{I I}\right)}}{\left(a_{I I}-c_{I I}\right)}}{4\left(a_{I}-c_{I}\right)^{2}}+$

$+\frac{1}{4\left(a_{I I}-c_{I I}\right)}$ 


$$
\begin{aligned}
& \left(\operatorname{Re}\left(\lambda_{1}\right)<0 \wedge \operatorname{Re}\left(\lambda_{2}\right)<0\right) \Leftrightarrow \\
& \left(a_{I I}<a_{I} \wedge c_{I}>a_{I} \wedge c_{I I}>a_{I I}\right) \vee\left(a_{I I}=a_{I} \wedge c_{I}>a_{I I} \wedge c_{I I}>a_{I I}\right) \vee \\
& \left(a_{I I}>a_{I} \wedge\left(\left(a_{I}<c_{I}<a_{I I} \wedge a_{I I}<c_{I I}<\frac{a_{I}^{2}-2 c_{I} \cdot a_{I}+4 a_{I I}^{2}+c_{I}^{2}-4 a_{I I} \cdot c_{I}}{4 a_{I I}-4 c_{I}}\right) \vee\right.\right. \\
& \left.\left.\vee\left(c_{I} \geq a_{I I} \wedge c_{I I}>a_{I I}\right)\right)\right) \\
& \left(\operatorname{Re}\left(\lambda_{1}\right)>0 \vee \operatorname{Re}\left(\lambda_{2}\right)>0\right) \Leftrightarrow \\
& \left(a_{I I}<a_{I} \wedge\left(c_{I}<a_{I} \vee\left(c_{I}>a_{I} \wedge \frac{a_{I}^{2}-2 c_{I} \cdot a_{I}+4 a_{I I}^{2}+c_{I}^{2}-4 a_{I I} \cdot c_{I}}{4 a_{I I}-4 c_{I}}<c_{I I}<a_{I I}\right)\right)\right) \vee \\
& \vee\left(a_{I I}=a_{I} \wedge\left(c_{I}<a_{I I} \vee\left(c_{I}>a_{I I} \wedge \frac{a_{I}^{2}-2 c_{I} \cdot a_{I}+4 a_{I I}^{2}+c_{I}^{2}-4 a_{I I} \cdot c_{I}}{4 a_{I I}-4 c_{I}}<c_{I I}<a_{I I}\right)\right)\right) \vee \\
& \vee\left(a_{I I}>a_{I} \wedge\left(c_{I}<a_{I} \vee\left(a_{I}<c_{I} \leq a_{I I} \wedge c_{I I}<a_{I I}\right) \vee\right.\right. \\
& \left.\left.\vee\left(c_{I}>a_{I I} \wedge \frac{a_{I}^{2}-2 c_{I} \cdot a_{I}+4 a_{I I}^{2}+c_{I}^{2}-4 a_{I I} \cdot c_{I}}{4 a_{I I}-4 c_{I}}<c_{I I}<a_{I I}\right)\right)\right) \\
& \text { - } y=-\frac{a_{I}+2 a_{I I}-c_{I}-2 c_{I I}+\sqrt{a_{I}^{2}-2 c_{I} \cdot a_{I}+4 a_{I I}^{2}-4 a_{I I}\left(c_{I}+c_{I I}\right)+c_{I}\left(c_{I}+4 c_{I I}\right)}}{2\left(a_{I}-c_{I}\right)\left(a_{I I}-c_{I I}\right) \mu} \\
& \mathrm{e} z=-\frac{1}{\left(a_{I}-c_{I}\right) \mu} \\
& \lambda_{1}=\frac{1}{2\left(a_{I}-c_{I}\right)}, \\
& \lambda_{2}=\frac{4\left(a_{I I}-c_{I}\right)+\frac{\left(c_{I}-a_{I}\right) \sqrt{a_{I}^{2}-2 c_{I} \cdot a_{I}+4 a_{I I}^{2}-4 a_{I I}\left(c_{I}+c_{I I}\right)+c_{I}\left(c_{I}+4 c_{I I}\right)}}{\left(a_{I I}-c_{I I}\right)}}{4\left(a_{I}-c_{I}\right)^{2}}+ \\
& +\frac{1}{4\left(a_{I I}-c_{I I}\right)}
\end{aligned}
$$




$$
\begin{aligned}
& \left(\operatorname{Re}\left(\lambda_{1}\right)<0 \wedge \operatorname{Re}\left(\lambda_{2}\right)<0\right) \Leftrightarrow \\
& \left(a_{I I}<a_{I} \wedge c_{I}>a_{I} \wedge\left(\frac{a_{I}^{2}-2 c_{I} \cdot a_{I}+4 a_{I I}^{2}+c_{I}^{2}-4 a_{I I} \cdot c_{I}}{4 a_{I I}-4 c_{I}}<c_{I I}<a_{I I} \vee c_{I I}>a_{I I}\right)\right) \vee \\
& \vee\left(a_{I I} \geq a_{I} \wedge c_{I}>a_{I I} \wedge\left(\frac{a_{I}^{2}-2 c_{I} \cdot a_{I}+4 a_{I I}^{2}+c_{I}^{2}-4 a_{I I} \cdot c_{I}}{4 a_{I I}-4 c_{I}}<c_{I I}<a_{I I} \vee c_{I I}>a_{I I}\right)\right) \\
& \left(\operatorname{Re}\left(\lambda_{1}\right)>0 \vee \operatorname{Re}\left(\lambda_{2}\right)>0\right) \Leftrightarrow \\
& \left(a_{I}<0 \wedge\left(( a _ { I I } \leq a _ { I } \wedge c _ { I } < a _ { I } ) \vee \left(a_{I I}>a_{I} \wedge\left(c_{I}<a_{I} \vee\left(a_{I}<c_{I}<a_{I I} \wedge\right.\right.\right.\right.\right. \\
& \left.\left.\left.\left.\left.\wedge\left(c_{I I}<a_{I I} \vee a_{I I}<c_{I I}<\frac{a_{I}^{2}-2 c_{I} \cdot a_{I}+4 a_{I I}^{2}+c_{I}^{2}-4 a_{I I} \cdot c_{I}}{4 a_{I I}-4 c_{I}}\right)\right)\right)\right)\right)\right) \vee \\
& \vee\left(a_{I} \geq 0 \wedge\left(( a _ { I I } < a _ { I } \wedge c _ { I } < a _ { I } ) \vee ( a _ { I I } = a _ { I } \wedge c _ { I } < a _ { I } ) \vee \left(a_{I I}>a_{I} \wedge\right.\right.\right. \\
& \wedge\left(c_{I}<a_{I} \vee\left(a_{I}<c_{I}<a_{I I} \wedge\right.\right. \\
& \left.\left.\left.\left.\left.\wedge\left(c_{I I}<a_{I I} \vee a_{I I}<c_{I I}<\frac{a_{I}^{2}-2 c_{I} \cdot a_{I}+4 a_{I I}^{2}+c_{I}^{2}-4 a_{I I} \cdot c_{I}}{4 a_{I I}-4 c_{I}}\right)\right)\right)\right)\right)\right)
\end{aligned}
$$

A interpretação analítica e gráfica dos resultados apresentados será realizada no capítulo VI. A seguir, na próxima seção, será realizado um estudo equivalente para os pontos $P_{1,2}$.

5.3 ESTUDO DOS PONTOS $P_{1,2}=\left(+\frac{\pi}{2}, 0,0,+\frac{\pi}{2}+\arccos \left[ \pm \sqrt{1-\left(\frac{\Omega}{\mu_{I I}}\right)^{2}}\right], 0,0\right)$

\subsubsection{ANÁLISE LINEAR DA ESTABILIDADE}

Considere-se a seguinte expansão em série de Taylor das funções seno e co-

$$
\begin{aligned}
& \text { seno em torno } x_{1}^{*}=+\frac{\pi}{2} \text { e } x_{4}^{*}=+\frac{\pi}{2}+\arccos \left[ \pm \sqrt{1-\left(\frac{\Omega}{\mu_{I I}}\right)^{2}}\right] \\
& \qquad \operatorname{sen} x_{1} \approx 1-\frac{1}{2}\left(x_{1}-\frac{\pi}{2}\right)^{2}+\ldots \text { e } \cos x_{1} \approx-\left(x_{1}-\frac{\pi}{2}\right)+\frac{1}{6}\left(x_{1}-\frac{\pi}{2}\right)^{3} \ldots,
\end{aligned}
$$




$$
\begin{aligned}
& \operatorname{sen} x_{4} \approx \cos \left(\frac{\pi}{2}+\rho_{I I}\right)-\operatorname{sen}\left(\frac{\pi}{2}+\rho_{I I}\right) \cdot\left(x_{4}-\left(\frac{\pi}{2}+\rho_{I I}\right)\right)+\ldots \mathrm{e} \\
& \cos x_{4} \approx-\operatorname{sen}\left(\frac{\pi}{2}+\rho_{I I}\right)-\cos \left(\frac{\pi}{2}+\rho_{I I}\right) \cdot\left(x_{4}-\left(\frac{\pi}{2}+\rho_{I I}\right)\right)+\ldots,
\end{aligned}
$$

Sendo que em (5.31) tem-se $\rho_{I I}=+\arccos \left[ \pm \sqrt{1-\left(\frac{\Omega}{\mu_{I I}}\right)^{2}}\right]$

Em seguida, considere-se a seguinte mudança de coordenadas:

$$
\bar{x}_{1}=x_{1}-\frac{\pi}{2}, \bar{x}_{2}=x_{2}, \bar{x}_{3}=x_{3}, \bar{x}_{4}=x_{4}-\left(\frac{\pi}{2}+\rho_{I I}\right), \bar{x}_{5}=x_{5} \text { e } \bar{x}_{6}=x_{6}
$$

A partir de (5.31) e (5.32), tem-se:

- $\cos \left(x_{4}-x_{1}\right)=\cos x_{4} \cos x_{1}+\operatorname{sen} x_{4} \operatorname{sen} x_{1}=$

$$
=\left(1-\frac{\bar{x}_{1}^{2}}{2}\right)\left( \pm \sqrt{1-\frac{\Omega^{2}}{\mu_{I I}^{2}}}-\frac{\Omega}{\mu_{I I}} \bar{x}_{4}\right)+\left(-\bar{x}_{1}+\frac{\bar{x}_{1}^{3}}{6}\right)\left(\mp \sqrt{1-\frac{\Omega^{2}}{\mu_{I I}^{2}}} \bar{x}_{4}-\frac{\Omega}{\mu_{I I}}\right)
$$

- $\operatorname{sen}\left(x_{4}-x_{1}\right)=\operatorname{sen} x_{4} \cos x_{1}-\operatorname{sen} x_{1} \cos x_{4}=$

$$
=\left(-\bar{x}_{1}+\frac{\bar{x}_{1}^{3}}{6}\right)\left( \pm \sqrt{1-\frac{\Omega^{2}}{\mu_{I I}^{2}}}-\frac{\Omega}{\mu_{I I}} \bar{x}_{4}\right)-\left(1-\frac{\bar{x}_{1}^{2}}{2}\right)\left(\mp \sqrt{1-\frac{\Omega^{2}}{\mu_{I I}^{2}}} \bar{x}_{4}-\frac{\Omega}{\mu_{I I}}\right)
$$

A seguir, considerando que $\mu_{I I}=m \cdot \mu_{I},(m>1, m \in \mathbb{R})$, define-se o seguinte parâmetro:

$$
k=\sqrt{m^{2}-1}, \text { ou seja, } \mu_{I} \cdot k=\sqrt{\mu_{I I}^{2}-\mu_{I}^{2}}
$$

Utilizando as expressões de (5.33), considerando que $\Omega=\mu_{I}<\mu_{I I}$ e o parâmetro $k$ definido em (5.34), reescreve-se o sistema (5.5), já transladado para a origem: 


$$
\left\{\begin{array}{l}
\dot{\bar{x}}_{1}=\bar{x}_{2} \\
\dot{\bar{x}}_{2}=\bar{x}_{3} \\
\dot{\bar{x}}_{3}=-\frac{c_{I}}{b_{I}} \bar{x}_{3}-\frac{1}{b_{I}} \bar{x}_{2}+\frac{\mu_{I} \cdot a_{I}}{b_{I}} \bar{x}_{1}+\frac{\mu_{I}}{2 b_{I}} \bar{x}_{1}^{2}-\frac{\mu_{I} \cdot a_{I}}{6 b_{I}} \bar{x}_{1}^{3} \cdot \bar{x}_{2} \\
\dot{\bar{x}}_{4}=x_{5} \\
\dot{\bar{x}}_{5}=x_{6} \\
\dot{\bar{x}}_{6}=-\frac{c_{I I}}{b_{I I}} \bar{x}_{6}-\frac{1}{b_{I I}} \bar{x}_{5} \mp \frac{k \cdot \mu_{I}}{6 b_{I I}} \bar{x}_{1}^{3}-\frac{a_{I I} \cdot \mu_{I}}{6 b_{I I}} \bar{x}_{2} \cdot \bar{x}_{1}^{3}+\frac{\mu_{I}}{6 b_{I I}} \bar{x}_{4} \cdot \bar{x}_{1}^{3} \mp \frac{a_{I I} \cdot k \cdot \mu_{I}}{6 b_{I I}} \bar{x}_{2} \cdot \bar{x}_{4} \cdot \bar{x}_{1}^{3}+ \\
+\frac{a_{I I} \cdot \mu_{I}}{6 b_{I I}} \bar{x}_{5} \cdot \bar{x}_{1}^{3} \pm \frac{a_{I I} \cdot k \cdot \mu_{I}}{6 b_{I I}} \bar{x}_{4} \cdot \bar{x}_{5} \cdot \bar{x}_{1}^{3}+\frac{\mu_{I}}{2 b_{I}} \bar{x}_{1}^{2} \mp \frac{a_{I I} \cdot k \cdot \mu_{I}}{2 b_{I I}} \bar{x}_{2} \cdot \bar{x}_{1}^{2} \pm \frac{k \cdot \mu_{I}}{2 b_{I I}} \bar{x}_{4} \cdot \bar{x}_{1}^{2}+ \\
+\frac{a_{I I} \cdot \mu_{I}}{2 b_{I I}} \bar{x}_{2} \cdot \bar{x}_{4} \cdot \bar{x}_{1}^{2} \pm \frac{a_{I I} \cdot k \cdot \mu_{I}}{2 b_{I I}} \bar{x}_{5} \cdot \bar{x}_{1}^{2}-\frac{a_{I I} \cdot \mu_{I}}{2 b_{I I}} \bar{x}_{4} \cdot \bar{x}_{5} \cdot \bar{x}_{1}^{2} \pm \frac{k \cdot \mu_{I}}{b_{I I}} \bar{x}_{1}+ \\
+\frac{a_{I I} \cdot \mu_{I}}{b_{I I}} \bar{x}_{2} \cdot \bar{x}_{1}-\frac{\mu_{I}}{b_{I I}} \bar{x}_{4} \cdot \bar{x}_{1} \pm \frac{a_{I I} \cdot k \cdot \mu_{I}}{b_{I I}} \bar{x}_{2} \cdot \bar{x}_{4} \cdot \bar{x}_{1}-\frac{a_{I I} \cdot \mu_{I}}{b_{I I}} \bar{x}_{5} \cdot \bar{x}_{1} \mp \\
\mp \frac{a_{I I} \cdot k \cdot \mu_{I}}{b_{I I}} \bar{x}_{4} \cdot \bar{x}_{5} \cdot \bar{x}_{1} \pm \frac{a_{I I} \cdot k \cdot \mu_{I}}{b_{I I}} \bar{x}_{2} \mp \frac{k \cdot \mu_{I}}{b_{I I}} \bar{x}_{4}-\frac{a_{I I} \cdot \mu_{I}}{b_{I I}} \bar{x}_{2} \cdot \bar{x}_{4} \mp \\
\mp \frac{a_{I I} \cdot k \cdot \mu_{I}}{b_{I I}} \bar{x}_{5}+\frac{a_{I I} \cdot \mu_{I}}{b_{I I}} \bar{x}_{5} \cdot \bar{x}_{4}
\end{array}\right.
$$

Caso se transforme o parâmetro $\mu_{I}$ em variável de estado, através da inclusão de uma equação diferencial sem dinâmica, ou seja, $\dot{\mu}_{I}=0$, a matriz Jacobiana de (5.35), calculada nos pontos de equilíbrio $P_{1,2}=\left(+\frac{\pi}{2}, 0,0,+\frac{\pi}{2}+\rho_{I I}, 0,0\right)$, é:

$$
J_{P_{1,2}}=\left[\begin{array}{cccccc}
0 & 1 & 0 & 0 & 0 & 0 \\
0 & 0 & 1 & 0 & 0 & 0 \\
0 & -\frac{1}{b_{I}} & -\frac{c_{I}}{b_{I}} & 0 & 0 & 0 \\
0 & 0 & 0 & 0 & 1 & 0 \\
0 & 0 & 0 & 0 & 0 & 1 \\
0 & 0 & 0 & 0 & -\frac{1}{b_{I I}} & -\frac{c_{I I}}{b_{I I}}
\end{array}\right]
$$


A matriz (5.36) é idêntica à matriz (5.9). Dessa forma, todos os resultados pertinentes ao estudo da estabilidade do ponto $P$ podem ser aqui aplicados da mesma forma. Para proceder ao estudo da estabilidade, será aplicado o TVC.

\subsubsection{ANÁLISE VIA TVC}

O sistema (5.35) pode ser representado matricialmente como $\dot{\bar{X}}=J_{P} \cdot \bar{X}+\bar{F}$, ou seja:

$$
\begin{aligned}
& {\left[\begin{array}{c}
\dot{\bar{x}}_{1} \\
\dot{\bar{x}}_{2} \\
\dot{\bar{x}}_{3} \\
\dot{\bar{x}}_{4} \\
\dot{\bar{x}}_{5} \\
\dot{\bar{x}}_{6}
\end{array}\right]=\left[\begin{array}{cccccc}
0 & 1 & 0 & 0 & 0 & 0 \\
0 & 0 & 1 & 0 & 0 & 0 \\
0 & -\frac{1}{b_{I}} & -\frac{c_{I}}{b_{I}} & 0 & 0 & 0 \\
0 & 0 & 0 & 0 & 1 & 0 \\
0 & 0 & 0 & 0 & 0 & 1 \\
0 & 0 & 0 & 0 & -\frac{1}{b_{I I}} & -\frac{c_{I I}}{b_{I I}}
\end{array}\right] \cdot\left[\begin{array}{c}
\bar{x}_{1} \\
\bar{x}_{2} \\
\bar{x}_{3} \\
\bar{x}_{4} \\
\bar{x}_{5} \\
\bar{x}_{6}
\end{array}\right]+}
\end{aligned}
$$

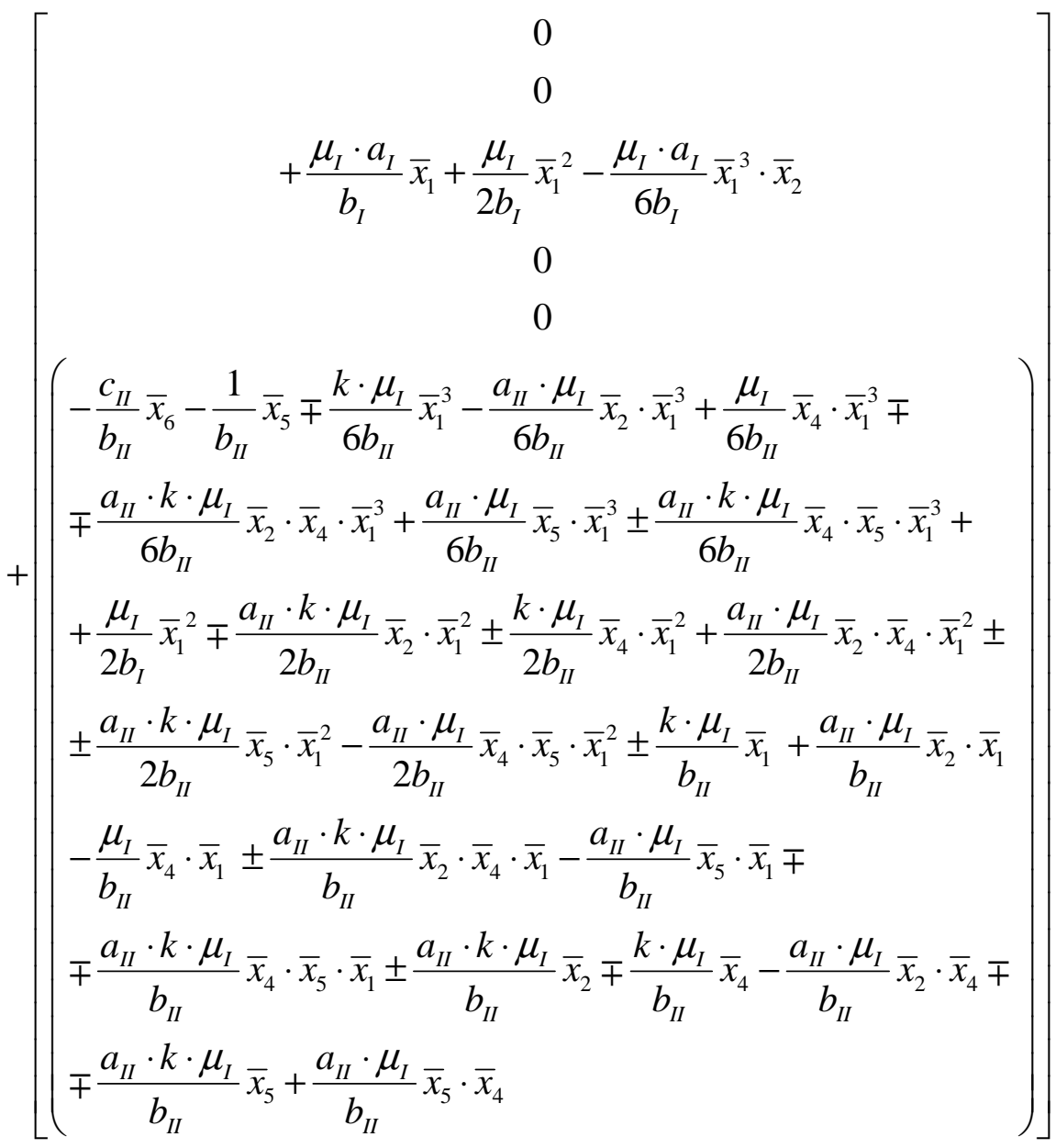


e $\dot{\mu}_{I}=0$

Conforme requerido pelo TVC, o sistema (5.38) precisa ser reescrito na forma canônica de Jordan. Para isso, define-se um novo conjunto de variáveis de estado $V=\left(v_{1}, v_{2}, v_{3}, v_{4}, v_{5}, v_{6}\right)^{T}$ de modo que $\bar{X}=M \cdot V$, sendo $M$ uma base composta pelos autovetores de $J_{P}$ de (5.10). Assim, é possível se estabelecer a seguinte relação entre as variáveis de estado $X$ e $V$ :

$$
\begin{aligned}
& \bar{x}_{1}=v_{2}+\frac{1}{2}\left(c_{I} \cdot \delta_{I}-2 b_{I}\right) v_{3}+\frac{1}{2}\left(c_{I} \cdot \varepsilon_{I}-2 b_{I}\right) v_{4} \\
& \bar{x}_{2}=\frac{1}{2} \delta_{I} \cdot v_{3}-\frac{1}{2} \varepsilon_{I} \cdot v_{4} \\
& \bar{x}_{3}=v_{3}+v_{4} \\
& \bar{x}_{4}=v_{1}+\frac{1}{2}\left(c_{I I} \cdot \delta_{I I}-2 b_{I I}\right) v_{5}+\frac{1}{2}\left(c_{I I} \cdot \varepsilon_{I I}-2 b_{I I}\right) v_{6} \\
& \bar{x}_{5}=\frac{1}{2} \delta_{I I} \cdot v_{5}-\frac{1}{2} \varepsilon_{I I} \cdot v_{6} \\
& \bar{x}_{6}=v_{5}+v_{6}
\end{aligned}
$$

Sendo que em (5.38), tem-se $\delta=c-\sqrt{c^{2}-4 b}$ e $\varepsilon=c+\sqrt{c^{2}-4 b}$.

Dessa forma, é possível fazer $V=\left[M^{-1} \cdot J_{P} \cdot M\right] \cdot V+M^{-1} \cdot \bar{F}$ e $\dot{\mu}_{I}=0$, ou seja:

$$
\left[\begin{array}{l}
\dot{v}_{1} \\
\dot{v}_{2} \\
\dot{v}_{3} \\
\dot{v}_{4} \\
\dot{v}_{5} \\
\dot{v}_{6}
\end{array}\right]=\left[\begin{array}{cccccc}
\lambda_{1} & 0 & 0 & 0 & 0 & 0 \\
0 & \lambda_{2} & 0 & 0 & 0 & 0 \\
0 & 0 & \lambda_{3} & 0 & 0 & 0 \\
0 & 0 & 0 & \lambda_{4} & 0 & 0 \\
0 & 0 & 0 & 0 & \lambda_{5} & 0 \\
0 & 0 & 0 & 0 & 0 & \lambda_{6}
\end{array}\right] \cdot\left[\begin{array}{c}
v_{1} \\
v_{2} \\
v_{3} \\
v_{4} \\
v_{5} \\
v_{6}
\end{array}\right]+\left[\begin{array}{l}
f_{1} \\
f_{2} \\
f_{3} \\
f_{4} \\
f_{5} \\
f_{6}
\end{array}\right] \text { e } \dot{\mu}_{I}=0
$$

Em (5.39), tem-se que:

- $\lambda_{1}=0$ 
- $\lambda_{2}=0$

- $\lambda_{3}=-\frac{\left(c_{I}+\sqrt{c_{I}^{2}-4 b_{I}}\right)}{2 b_{I}}$

- $\lambda_{4}=\frac{\left(\sqrt{c_{I}^{2}-4 b_{I}}-c_{I}\right)}{2 b_{I}}$

- $\lambda_{5}=-\frac{\left(c_{I I}+\sqrt{c_{I I}^{2}-4 b_{I I}}\right)}{2 b_{I I}}$

- $\lambda_{6}=\frac{\left(\sqrt{c_{I I}^{2}-4 b_{I I}}-c_{I I}\right)}{2 b_{I I}}$

- $\left[\begin{array}{l}f_{1} \\ f_{2} \\ f_{3} \\ f_{4} \\ f_{5} \\ f_{6}\end{array}\right]=M^{-1} \cdot \bar{F}$

Por questão de simplicidade, optou-se por omitir os termos não-lineares $f_{1}$, $f_{2}, f_{3}, f_{4}, f_{5}$ e $f_{6}$ devido a sua extensão.

Então, de acordo com o Teorema da Variedade Central (Guckenheimer e Holmes, 1983; Wiggins, 1990; Monteiro, 2002), procura-se a variedade central $W^{C}$ que seja tangente subespaço central $E^{C}$ no ponto de equilíbrio $P$. Assim, para $y, z$ e $\mu_{I}$ suficientemente pequenos, tem-se:

$$
\begin{aligned}
W^{c}(0)=\{ & \left(v_{1}, v_{2}, v_{3}, v_{4}, v_{5}, v_{6}\right) \in \Re^{4} / v_{1}=y, v_{2}=z, \\
& v_{3}=h_{1}\left(y, z, \mu_{I}\right), v_{4}=h_{2}\left(y, z, \mu_{I}\right), \\
& v_{5}=h_{3}\left(y, z, \mu_{I}\right), v_{6}=h_{4}\left(y, z, \mu_{I}\right), \\
& \left.h_{j}(0,0,0)=0, D h_{j}(0,0,0)=0, j=1,2,3,4\right\}
\end{aligned}
$$

Considere-se então, os seguintes polinômios estimadores compostos por monômios do segundo grau $h_{1}\left(y, z, \mu_{I}\right), h_{2}\left(y, z, \mu_{I}\right), h_{3}\left(y, z, \mu_{I}\right)$ e $h_{4}\left(y, z, \mu_{I}\right)$ : 


$$
\begin{aligned}
& h_{1}\left(y, z, \mu_{I}\right)=\alpha_{1} \cdot y^{2}+\alpha_{2} \cdot z^{2}+\alpha_{3} \cdot \mu_{I}^{2}+\alpha_{4} \cdot y \cdot z+\alpha_{5} \cdot y \cdot \mu_{I} \\
& \quad+\alpha_{6} \cdot z \cdot \mu_{I},\left(\alpha_{j} \in \mathbb{R}, j=1, \ldots, 7\right) \\
& h_{2}\left(y, z, \mu_{I}\right)=\beta_{1} \cdot y^{2}+\beta_{2} \cdot z^{2}+\beta_{3} \cdot \mu_{I}^{2}+\beta_{4} \cdot y \cdot z+\beta_{5} \cdot y \cdot \mu_{I} \\
& \quad+\beta_{6} \cdot z \cdot \mu_{I},\left(\beta_{j} \in \mathbb{R}, j=1, \ldots, 7\right) \\
& h_{3}\left(y, z, \mu_{I}\right)=\gamma_{1} \cdot y^{2}+\gamma_{2} \cdot z^{2}+\gamma_{3} \cdot \mu_{I}^{2}+\gamma_{4} \cdot y \cdot z+\gamma_{5} \cdot y \cdot \mu_{I} \\
& \quad+\gamma_{6} \cdot z \cdot \mu_{I},\left(\gamma_{j} \in \mathbb{R}, j=1, \ldots, 7\right) \\
& h_{3}\left(y, z, \mu_{I}\right)=\theta_{1} \cdot y^{2}+\theta_{2} \cdot z^{2}+\theta_{3} \cdot \mu_{I}^{2}+\theta_{4} \cdot y \cdot z+\theta_{5} \cdot y \cdot \mu_{I} \\
& \quad+\theta_{6} \cdot z \cdot \mu_{I},\left(\theta_{j} \in \mathbb{R}, j=1, \ldots, 7\right)
\end{aligned}
$$

Pelo teorema de Carr (Carr, 1981; Guckenheimer e Holmes, 1983; Wiggins, 1990), a variedade central $W^{c}$ deve satisfazer $N\left(h\left(y, z, \mu_{I}\right)\right)=0$. Portanto, tem-se:

$$
\begin{aligned}
& \frac{\partial h_{1}\left(y, z, \mu_{I}\right)}{\partial y}\left[\lambda_{1} \cdot x+f_{1}\left(y, z, \mu_{I}, h_{1}, h_{2}, h_{3}, h_{4}\right)\right]+ \\
& \quad+\frac{\partial h_{1}\left(y, z, \mu_{I}\right)}{\partial z}\left[\lambda_{2} \cdot x+f_{2}\left(y, z, \mu_{I}, h_{1}, h_{2}, h_{3}, h_{4}\right)\right]- \\
& \quad-\lambda_{3} \cdot h_{1}\left(y, z, \mu_{I}\right)-f_{3}\left(y, z, \mu_{I}, h_{1}, h_{2}, h_{3}, h_{4}\right)=0
\end{aligned}
$$

$$
\begin{aligned}
& \frac{\partial h_{2}\left(y, z, \mu_{I}\right)}{\partial z}\left[\lambda_{2} \cdot x+f_{2}\left(y, z, \mu_{I}, h_{1}, h_{2}, h_{3}, h_{4}\right)\right]+ \\
& \quad+\frac{\partial h_{2}\left(y, z, \mu_{I}\right)}{\partial z}\left[\lambda_{2} \cdot x+f_{2}\left(y, z, \mu_{I}, h_{1}, h_{2}, h_{3}, h_{4}\right)\right]- \\
& \quad-\lambda_{4} \cdot h_{2}\left(y, z, \mu_{I}\right)-f_{4}\left(y, z, \mu_{I}, h_{1}, h_{2}, h_{3}, h_{4}\right)=0
\end{aligned}
$$

$$
\begin{aligned}
& \frac{\partial h_{3}\left(y, z, \mu_{I}\right)}{\partial y}\left[\lambda_{1} \cdot x+f_{1}\left(y, z, \mu_{I}, h_{1}, h_{2}, h_{3}, h_{4}\right)\right]+ \\
& \quad+\frac{\partial h_{3}\left(y, z, \mu_{I}\right)}{\partial z}\left[\lambda_{2} \cdot x+f_{2}\left(y, z, \mu_{I}, h_{1}, h_{2}, h_{3}, h_{4}\right)\right]- \\
& \quad-\lambda_{4} \cdot h_{5}\left(y, z, \mu_{I}\right)-f_{4}\left(y, z, \mu_{I}, h_{1}, h_{2}, h_{3}, h_{4}\right)=0
\end{aligned}
$$

$$
\begin{aligned}
& \frac{\partial h_{4}\left(y, z, \mu_{I}\right)}{\partial z}\left[\lambda_{2} \cdot x+f_{2}\left(y, z, \mu_{I}, h_{1}, h_{2}, h_{3}, h_{4}\right)\right] \\
& \quad+\frac{\partial h_{4}\left(y, z, \mu_{I}\right)}{\partial z}\left[\lambda_{2} \cdot x+f_{2}\left(y, z, \mu_{I}, h_{1}, h_{2}, h_{3}, h_{4}\right)\right]- \\
& -\lambda_{6} \cdot h_{4}\left(y, z, \mu_{I}\right)-f_{6}\left(y, z, \mu_{I}, h_{1}, h_{2}, h_{3}, h_{4}\right)=0
\end{aligned}
$$


Utilizando (5.39) e (5.41) em (5.42) e resolvendo o sistema de equações, é possível obter, igualando-se a zero os termos de igual potência, os coeficientes de $h_{1}\left(y, z, \mu_{I}\right), h_{2}\left(y, z, \mu_{I}\right), h_{3}\left(y, z, \mu_{I}\right)$ e $h_{4}\left(y, z, \mu_{I}\right)$ :

$$
\begin{aligned}
& y^{2}: \alpha_{1}=0, \beta_{1}=0, \gamma_{1}=0, \theta_{1}=0 \\
& z^{2}: \alpha_{2}=0, \beta_{2}=0, \gamma_{2}=0, \theta_{2}=0 \\
& \mu_{I}^{2}: \alpha_{3}=0, \beta_{3}=0, \gamma_{3}=0, \theta_{3}=0 \\
& y \cdot z: \alpha_{4}=0, \beta_{4}=0, \gamma_{4}=0, \theta_{4}=0 \\
& y \cdot \mu_{I}: \alpha_{5}=0, \beta_{5}=0, \gamma_{5}=\mp \frac{\sqrt{c_{I I}^{2}-4 b_{I I}}}{c_{I I}^{2}-4 b_{I I}} k, \theta_{5}= \pm \frac{\sqrt{c_{I I}^{2}-4 b_{I I}}}{c_{I I}^{2}-4 b_{I I}} k \\
& z \cdot \mu_{I}: \alpha_{6}=0, \beta_{6}=0, \gamma_{6}= \pm \frac{\sqrt{c_{I I}^{2}-4 b_{I I}}}{c_{I I}^{2}-4 b_{I I}} k, \theta_{6}=\mp \frac{\sqrt{c_{I I}^{2}-4 b_{I I}}}{c_{I I}^{2}-4 b_{I I}} k
\end{aligned}
$$

Com os coeficientes (5.43) dos polinômios de (5.41), pode-se obter, através do sistema (5.39), o campo de vetores restrito à variedade central, desprezando-se os termos de ordem superior:

$$
\left\{\begin{aligned}
\dot{y}= & \mp \frac{\mu_{I}}{2}\left\{ \pm 2(y-z) \mu_{I}\left[c_{I I}+a_{I I}\left(c_{I I}(y-z) \mu_{I}-1\right)\right] k^{2}+\right. \\
& \left.+2(y-z)\left[a_{I I}(y-z) \mu_{I}+c_{I I} \cdot z \cdot \mu_{I}+1\right] k \pm z(y-z)\right\} \\
\dot{z}= & \frac{\mu_{I}}{2} z^{2}
\end{aligned}\right.
$$

Para obter os pontos de equilíbrio de (5.44), basta fazer:

$$
\left\{\begin{array}{l}
\dot{y}=0 \\
\dot{z}=0
\end{array}\right.
$$

De (5.19) se obtém os seguintes possíveis valores para y e $z$ :

- $y=0$ e $z=0$

- $y=\frac{a_{I I} \cdot k \cdot \mu_{I}-c_{I I} \cdot k \cdot \mu_{I} \mp 1}{a_{I I} \cdot \mu_{I}\left(c_{I I} \cdot k \cdot \mu_{I} \pm 1\right)}$ e $z=0$, sendo $a_{I I} \cdot \mu_{I}\left(c_{I I} \cdot k \cdot \mu_{I} \pm 1\right) \neq 0$ 
Para o estudo das estabilidades dos pontos de equilíbrio de (5.46), é preciso obter a matriz Jacobiana de (5.44):

$$
J_{P}=\left[\begin{array}{ll}
\left.\frac{\partial \dot{y}}{\partial y}\right|_{P} & \left.\frac{\partial \dot{y}}{\partial z}\right|_{P} \\
\left.\frac{\partial \dot{z}}{\partial y}\right|_{P} & \left.\frac{\partial \dot{z}}{\partial z}\right|_{P}
\end{array}\right]
$$

Sendo que em (5.47), tem-se:

$$
\begin{aligned}
& \frac{\partial \dot{y}}{\partial y}=\mu_{I}\left\{-z \mp k\left[c_{I I}( \pm k+z) \mu_{I}+a_{I I}\left(2 c_{I I}( \pm y \mp z) \mu_{I} \cdot k \mp k+2 y-2 z\right) \mu_{I}+1\right]\right\} \\
& \frac{\partial \dot{y}}{\partial z}=\mu_{I}\left\{-y+z+k\left[c_{I I}(k \mp y \pm 2 z) \mu_{I}+a_{I I}\left(2 c_{I I}(y-z) \mu_{I} \cdot k-k \pm 2 y \mp 2 z\right) \mu_{I} \pm 1\right]\right\} \\
& \frac{\partial \dot{z}}{\partial y}=0 \\
& \frac{\partial \dot{z}}{\partial z}=z \cdot \mu_{I}
\end{aligned}
$$

Em seguida, verifica-se a parte real dos autovalores para a matriz Jacobiana (5.47) calculada em cada ponto de equilíbrio:

- $y=0$ e $z=0$

$$
\begin{aligned}
& \lambda_{1}=0, \lambda_{2}=k \cdot \mu_{I}\left(\left(a_{I I}-c_{I I}\right) \cdot k \cdot \mu_{I} \mp 1\right) \\
& \left(\operatorname{Re}\left(\lambda_{2}\right)>0\right) \Leftrightarrow\left(c_{I I}<\frac{a_{I I} \cdot k \cdot \mu_{I} \mp 1}{k \cdot \mu_{I}}\right)
\end{aligned}
$$

- $y=\frac{a_{I I} \cdot k \cdot \mu_{I}-c_{I I} \cdot k \cdot \mu_{I} \mp 1}{a_{I I} \cdot \mu_{I}\left(c_{I I} \cdot k \cdot \mu_{I} \pm 1\right)}$ e $z=0$, sendo $a_{I I} \cdot \mu_{I}\left(c_{I I} \cdot k \cdot \mu_{I} \pm 1\right) \neq 0$

$$
\begin{aligned}
& \lambda_{1}=0, \lambda_{2}=k \cdot \mu_{I}\left(\left(c_{I I}-a_{I I}\right) \cdot k \cdot \mu_{I} \pm 1\right) \\
& \left(\operatorname{Re}\left(\lambda_{2}\right)>0\right) \Leftrightarrow\left(c_{I I}>\frac{a_{I I} \cdot k \cdot \mu_{I} \mp 1}{k \cdot \mu_{I}}\right)
\end{aligned}
$$


A interpretação analítica e gráfica dos resultados apresentados será realizada no capítulo VI. A seguir, na próxima seção, será realizado um estudo equivalente para os pontos $P_{3,4}$.

\subsection{ESTUDO DOS PONTOS}

$$
P_{3,4}=\left(\arccos \left[ \pm \sqrt{1-\left(\frac{\Omega}{\mu_{I}}\right)^{2}}\right], 0,0,+\frac{\pi}{2}+\arccos \left[ \pm \sqrt{1-\left(\frac{\Omega}{\mu_{I}}\right)^{2}}\right], 0,0\right)
$$

\subsubsection{ANÁLISE LINEAR DA ESTABILIDADE}

Considere-se a seguinte expansão em série de Taylor das funções seno e co-

$$
\begin{aligned}
& \text { seno em torno } x_{1}^{*}=\arccos \left[ \pm \sqrt{1-\left(\frac{\Omega}{\mu_{I}}\right)^{2}}\right] \text { e } x_{4}^{*}=+\frac{\pi}{2}+\arccos \left[ \pm \sqrt{1-\left(\frac{\Omega}{\mu_{I}}\right)^{2}}\right]: \\
& \operatorname{sen} x_{1} \approx+\operatorname{sen} \rho_{I}+\cos \rho_{I} \cdot\left(x_{1}-\rho_{I}\right)+\ldots, \cos x_{1} \approx+\cos \rho_{I}-\operatorname{sen} \rho_{I} \cdot\left(x_{1}-\rho_{I}\right)+\ldots \\
& \operatorname{sen} x_{4} \approx \cos \rho_{I}-\operatorname{sen} \rho_{I} \cdot\left(x_{4}-\left(\frac{\pi}{2}+\rho_{I}\right)\right)+\ldots \\
& \cos x_{4} \approx-\operatorname{sen} \rho_{I}-\cos \rho_{I} \cdot\left(x_{4}-\left(\frac{\pi}{2}+\rho_{I}\right)\right)+\ldots
\end{aligned}
$$

Sendo que em (5.50) tem-se: $\rho_{I}=+\arccos \left[ \pm \sqrt{1-\left(\frac{\Omega}{\mu_{I}}\right)^{2}}\right]$.

Em seguida, considere-se a seguinte mudança de coordenadas:

$$
\bar{x}_{1}=x_{1}-\left(x_{1}-\rho_{I}\right), \bar{x}_{2}=x_{2}, \bar{x}_{3}=x_{3}, \bar{x}_{4}=x_{4}-\left(\frac{\pi}{2}+\rho_{I}\right), \bar{x}_{5}=x_{5} \text { e } \bar{x}_{6}=x_{6}
$$

A partir de (5.50) e (5.51), tem-se:

- $\cos \left(x_{4}-x_{1}\right)=\cos x_{4} \cos x_{1}+\operatorname{sen} x_{4} \operatorname{sen} x_{1}=$ 


$$
\begin{aligned}
& =\left( \pm \sqrt{1-\frac{\Omega^{2}}{\mu_{I}^{2}}} \bar{x}_{1}+\frac{\Omega}{\mu_{I}}\right)\left( \pm \sqrt{1-\frac{\Omega^{2}}{\mu_{I}^{2}}}-\frac{\Omega}{\mu_{I}} \bar{x}_{4}\right)+ \\
& +\left( \pm \sqrt{1-\frac{\Omega^{2}}{\mu_{I}^{2}}}-\frac{\Omega}{\mu_{I}} \bar{x}_{1}\right)\left(\mp \sqrt{1-\frac{\Omega^{2}}{\mu_{I}^{2}}} \bar{x}_{4}-\frac{\Omega}{\mu_{I}}\right)
\end{aligned}
$$

- $\operatorname{sen}\left(x_{4}-x_{1}\right)=\operatorname{sen} x_{4} \cos x_{1}-\operatorname{sen} x_{1} \cos x_{4}=$

$$
\begin{aligned}
& =\left( \pm \sqrt{1-\frac{\Omega^{2}}{\mu_{I}^{2}}}-\frac{\Omega}{\mu_{I}} \bar{x}_{1}\right)\left( \pm \sqrt{1-\frac{\Omega^{2}}{\mu_{I}^{2}}}-\frac{\Omega}{\mu_{I}} \bar{x}_{4}\right)- \\
& -\left( \pm \sqrt{1-\frac{\Omega^{2}}{\mu_{I}^{2}}} \bar{x}_{1}+\frac{\Omega}{\mu_{I}}\right)\left(\mp \sqrt{1-\frac{\Omega^{2}}{\mu_{I}^{2}}} \bar{x}_{4}-\frac{\Omega}{\mu_{I}}\right)
\end{aligned}
$$

A seguir, considerando que $\mu_{I}=m \cdot \mu_{I I},(m>1, m \in \mathbb{R})$, define-se o seguinte parâmetro:

$$
k=\sqrt{m^{2}-1}, \text { ou seja, } \mu_{I I} \cdot k=\sqrt{\mu_{I}^{2}-\mu_{I I}^{2}}
$$

Utilizando as expressões de (5.52), considerando que $\Omega=\mu_{I I}<\mu_{I}$ e o parâmetro $k$ definido em (5.53), reescreve-se o sistema (5.5), já transladado para a origem:

$$
\left\{\begin{array}{l}
\dot{\bar{x}}_{1}=\bar{x}_{2} \\
\dot{\bar{x}}_{2}=\bar{x}_{3} \\
\dot{\bar{x}}_{3}=-\frac{c_{I}}{b_{I}} \bar{x}_{3}-\frac{1}{b_{I}} \bar{x}_{2} \mp \frac{k \cdot \mu_{I I} \cdot a_{I}}{b_{I}} \bar{x}_{2}+\frac{a_{I} \cdot k \cdot \mu_{I I}}{b_{I}} \bar{x}_{1} \cdot \bar{x}_{2}+\frac{\mu_{I I} \cdot k}{b_{I}} \bar{x}_{1} \\
\dot{\bar{x}}_{4}=x_{5} \\
\dot{\bar{x}}_{5}=x_{6} \\
\dot{\bar{x}}_{6}=-\frac{c_{I I}}{b_{I I}} \bar{x}_{6}-\frac{1}{b_{I I}} \bar{x}_{5}+\frac{a_{I I} \cdot \mu_{I I}}{b_{I I}} \bar{x}_{1} \cdot \bar{x}_{2}-\frac{a_{I I} \cdot \mu_{I I}}{b_{I I}} \bar{x}_{4} \cdot \bar{x}_{2}- \\
-\frac{\mu_{I I}}{b_{I I}} \bar{x}_{1} \cdot \bar{x}_{4}-\frac{a_{I I} \cdot \mu_{I I}}{b_{I I}} \bar{x}_{1} \cdot \bar{x}_{5}+\frac{a_{I I} \cdot \mu_{I I}}{b_{I I}} \bar{x}_{4} \cdot \bar{x}_{5}
\end{array}\right.
$$

Caso se transforme o parâmetro $\mu_{I I}$ em variável de estado, através da inclusão de uma equação diferencial sem dinâmica, ou seja, $\dot{\mu}_{I I}=0$, a matriz 
Jacobiana de (5.35), calculada nos pontos de equilíbrio $P_{3,4}=\left(\rho_{I}, 0,0,+\frac{\pi}{2}+\rho_{I}, 0,0\right)$, é:

$$
J_{P_{3,4}}=\left[\begin{array}{cccccc}
0 & 1 & 0 & 0 & 0 & 0 \\
0 & 0 & 1 & 0 & 0 & 0 \\
0 & -\frac{1}{b_{I}} & -\frac{c_{I}}{b_{I}} & 0 & 0 & 0 \\
0 & 0 & 0 & 0 & 1 & 0 \\
0 & 0 & 0 & 0 & 0 & 1 \\
0 & 0 & 0 & 0 & -\frac{1}{b_{I I}} & -\frac{c_{I I}}{b_{I I}}
\end{array}\right]
$$

A matriz (5.55) é idêntica à matriz (5.9). Dessa forma, todos os resultados pertinentes ao estudo da estabilidade do ponto $P$ podem ser aqui aplicados da mesma forma. Para proceder ao estudo da estabilidade, será aplicado o TVC.

\subsubsection{ANÁLISE VIA TVC}

O sistema (5.54) pode ser representado matricialmente como $\dot{\bar{X}}=J_{P_{3,4}} \cdot \bar{X}+\bar{F}$, ou seja:

$$
\left[\begin{array}{c}
\dot{\bar{x}}_{1} \\
\dot{\bar{x}}_{2} \\
\dot{\bar{x}}_{3} \\
\dot{\bar{x}}_{4} \\
\dot{\bar{x}}_{5} \\
\dot{\bar{x}}_{6}
\end{array}\right]=\left[\begin{array}{cccccc}
0 & 1 & 0 & 0 & 0 & 0 \\
0 & 0 & 1 & 0 & 0 & 0 \\
0 & -\frac{1}{b_{I}} & -\frac{c_{I}}{b_{I}} & 0 & 0 & 0 \\
0 & 0 & 0 & 0 & 1 & 0 \\
0 & 0 & 0 & 0 & 0 & 1 \\
0 & 0 & 0 & 0 & -\frac{1}{b_{I I}} & -\frac{c_{I I}}{b_{I I}}
\end{array}\right] \cdot\left[\begin{array}{c}
\bar{x}_{1} \\
\bar{x}_{2} \\
\bar{x}_{3} \\
\bar{x}_{4} \\
\bar{x}_{5} \\
\bar{x}_{6}
\end{array}\right]+
$$




$$
+\left[\begin{array}{c}
0 \\
0 \\
\mp \frac{k \cdot \mu_{I I} \cdot a_{I}}{b_{I}} \bar{x}_{2}+\frac{a_{I} \cdot k \cdot \mu_{I I}}{b_{I}} \bar{x}_{1} \cdot \bar{x}_{2}+\frac{\mu_{I I} \cdot k}{b_{I}} \bar{x}_{1} \\
0 \\
0 \\
\mathrm{e} \dot{\mu}_{I I}=0
\end{array}\right]
$$

Conforme requerido pelo TVC, o sistema (5.54) precisa ser reescrito na forma canônica de Jordan. Para isso, define-se um novo conjunto de variáveis de estado $V=\left(v_{1}, v_{2}, v_{3}, v_{4}, v_{5}, v_{6}\right)^{T}$ de modo que $\bar{X}=M \cdot V$, sendo $M$ uma base composta pelos autovetores de $J_{P}$ de (5.10). Assim, é possível se estabelecer a seguinte relação entre as variáveis de estado $X$ e $V$ :

$$
\begin{aligned}
& \bar{x}_{1}=v_{2}+\frac{1}{2}\left(c_{I} \cdot \delta_{I}-2 b_{I}\right) v_{3}+\frac{1}{2}\left(c_{I} \cdot \varepsilon_{I}-2 b_{I}\right) v_{4} \\
& \bar{x}_{2}=\frac{1}{2} \delta_{I} \cdot v_{3}-\frac{1}{2} \varepsilon_{I} \cdot v_{4} \\
& \bar{x}_{3}=v_{3}+v_{4} \\
& \bar{x}_{4}=v_{1}+\frac{1}{2}\left(c_{I I} \cdot \delta_{I I}-2 b_{I I}\right) v_{5}+\frac{1}{2}\left(c_{I I} \cdot \varepsilon_{I I}-2 b_{I I}\right) v_{6} \\
& \bar{x}_{5}=\frac{1}{2} \delta_{I I} \cdot v_{5}-\frac{1}{2} \varepsilon_{I I} \cdot v_{6} \\
& \bar{x}_{6}=v_{5}+v_{6}
\end{aligned}
$$

Sendo que em (5.57), tem-se $\delta=c-\sqrt{c^{2}-4 b}$ e $\varepsilon=c+\sqrt{c^{2}-4 b}$.

Dessa forma, é possível fazer $V=\left[M^{-1} \cdot J_{P} \cdot M\right] \cdot V+M^{-1} \cdot \bar{F}$ e $\dot{\mu}_{I I}=0$, ou seja: 


$$
\left[\begin{array}{l}
\dot{v}_{1} \\
\dot{v}_{2} \\
\dot{v}_{3} \\
\dot{v}_{4} \\
\dot{v}_{5} \\
\dot{v}_{6}
\end{array}\right]=\left[\begin{array}{cccccc}
\lambda_{1} & 0 & 0 & 0 & 0 & 0 \\
0 & \lambda_{2} & 0 & 0 & 0 & 0 \\
0 & 0 & \lambda_{3} & 0 & 0 & 0 \\
0 & 0 & 0 & \lambda_{4} & 0 & 0 \\
0 & 0 & 0 & 0 & \lambda_{5} & 0 \\
0 & 0 & 0 & 0 & 0 & \lambda_{6}
\end{array}\right] \cdot\left[\begin{array}{l}
v_{1} \\
v_{2} \\
v_{3} \\
v_{4} \\
v_{5} \\
v_{6}
\end{array}\right]+\left[\begin{array}{l}
f_{1} \\
f_{2} \\
f_{3} \\
f_{4} \\
f_{5} \\
f_{6}
\end{array}\right] \mathrm{e} \dot{\mu}_{I I}=0
$$

Em (5.58), tem-se que:

- $\lambda_{1}=0$

- $\lambda_{2}=0$

- $\lambda_{3}=-\frac{\left(c_{I}+\sqrt{c_{I}^{2}-4 b_{I}}\right)}{2 b_{I}}$

- $\lambda_{4}=\frac{\left(\sqrt{c_{I}^{2}-4 b_{I}}-c_{I}\right)}{2 b_{I}}$

- $\lambda_{5}=-\frac{\left(c_{I I}+\sqrt{c_{I I}^{2}-4 b_{I I}}\right)}{2 b_{I I}}$

- $\lambda_{6}=\frac{\left(\sqrt{c_{I I}^{2}-4 b_{I I}}-c_{I I}\right)}{2 b_{I I}}$

- $\left[\begin{array}{l}f_{1} \\ f_{2} \\ f_{3} \\ f_{4} \\ f_{5} \\ f_{6}\end{array}\right]=M^{-1} \cdot \bar{F}$

Por questão de simplicidade, optou-se por omitir os termos não-lineares $f_{1}$, $f_{2}, f_{3}, f_{4}, f_{5}$ e $f_{6}$ devido a sua extensão.

Então, de acordo com o Teorema da Variedade Central (Guckenheimer e Holmes, 1983; Wiggins, 1990; Monteiro, 2002), procura-se a variedade central $W^{C}$ 
que seja tangente subespaço central $E^{C}$ no ponto de equilíbrio $P$. Assim, para $y, z$ e $\mu_{I I}$ suficientemente pequenos, tem-se:

$$
\begin{aligned}
W^{c}(0)=\{ & \left(v_{1}, v_{2}, v_{3}, v_{4}, v_{5}, v_{6}\right) \in \mathfrak{R}^{4} / v_{1}=y, v_{2}=z, \\
& v_{3}=h_{1}\left(y, z, \mu_{I I}\right), v_{4}=h_{2}\left(y, z, \mu_{I I}\right), \\
& v_{5}=h_{3}\left(y, z, \mu_{I I}\right), v_{6}=h_{4}\left(y, z, \mu_{I I}\right), \\
& \left.h_{j}(0,0,0)=0, D h_{j}(0,0,0)=0, j=1,2,3,4\right\}
\end{aligned}
$$

Considere-se então, os seguintes polinômios estimadores compostos por monômios do segundo grau $h_{1}\left(y, z, \mu_{I I}\right), h_{2}\left(y, z, \mu_{I I}\right), h_{3}\left(y, z, \mu_{I I}\right)$ e $h_{4}\left(y, z, \mu_{I I}\right)$ :

$$
\begin{aligned}
& \quad h_{1}\left(y, z, \mu_{I I}\right)=\alpha_{1} \cdot y^{2}+\alpha_{2} \cdot z^{2}+\alpha_{3} \cdot \mu_{I I}{ }^{2}+\alpha_{4} \cdot y \cdot z+\alpha_{5} \cdot y \cdot \mu_{I I} \\
& \quad+\alpha_{6} \cdot z \cdot \mu_{I I},\left(\alpha_{j} \in \mathbb{R}, j=1, \ldots, 7\right) \\
& h_{2}\left(y, z, \mu_{I I}\right)=\beta_{1} \cdot y^{2}+\beta_{2} \cdot z^{2}+\beta_{3} \cdot \mu_{I I}^{2}+\beta_{4} \cdot y \cdot z+\beta_{5} \cdot y \cdot \mu_{I I} \\
& \quad+\beta_{6} \cdot z \cdot \mu_{I I},\left(\beta_{j} \in \mathbb{R}, j=1, \ldots, 7\right) \\
& h_{3}\left(y, z, \mu_{I I}\right)=\gamma_{1} \cdot y^{2}+\gamma_{2} \cdot z^{2}+\gamma_{3} \cdot \mu_{I I}^{2}+\gamma_{4} \cdot y \cdot z+\gamma_{5} \cdot y \cdot \mu_{I I} \\
& \quad+\gamma_{6} \cdot z \cdot \mu_{I I},\left(\gamma_{j} \in \mathbb{R}, j=1, \ldots, 7\right) \\
& h_{3}\left(y, z, \mu_{I I}\right)=\theta_{1} \cdot y^{2}+\theta_{2} \cdot z^{2}+\theta_{3} \cdot \mu_{I I}^{2}+\theta_{4} \cdot y \cdot z+\theta_{5} \cdot y \cdot \mu_{I I} \\
& \quad+\theta_{6} \cdot z \cdot \mu_{I I},\left(\theta_{j} \in \mathbb{R}, j=1, \ldots, 7\right)
\end{aligned}
$$

Pelo teorema de Carr (Carr, 1981; Guckenheimer e Holmes, 1983; Wiggins, 1990), a variedade central $W^{c}$ deve satisfazer $N\left(h\left(y, z, \mu_{I I}\right)\right)=0$. Portanto, tem-se:

$$
\begin{aligned}
& \frac{\partial h_{1}\left(y, z, \mu_{I I}\right)}{\partial y}\left[\lambda_{1} \cdot x+f_{1}\left(y, z, \mu_{I I}, h_{1}, h_{2}, h_{3}, h_{4}\right)\right]+ \\
& \quad+\frac{\partial h_{1}\left(y, z, \mu_{I I}\right)}{\partial z}\left[\lambda_{2} \cdot x+f_{2}\left(y, z, \mu_{I I}, h_{1}, h_{2}, h_{3}, h_{4}\right)\right]- \\
& \quad-\lambda_{3} \cdot h_{1}\left(y, z, \mu_{I I}\right)-f_{3}\left(y, z, \mu_{I I}, h_{1}, h_{2}, h_{3}, h_{4}\right)=0 \\
& \frac{\partial h_{2}\left(y, z, \mu_{I I}\right)}{\partial z}\left[\lambda_{2} \cdot x+f_{2}\left(y, z, \mu_{I I}, h_{1}, h_{2}, h_{3}, h_{4}\right)\right]+ \\
& \quad+\frac{\partial h_{2}\left(y, z, \mu_{I I}\right)}{\partial z}\left[\lambda_{2} \cdot x+f_{2}\left(y, z, \mu_{I I}, h_{1}, h_{2}, h_{3}, h_{4}\right)\right]- \\
& \quad-\lambda_{4} \cdot h_{2}\left(y, z, \mu_{I I}\right)-f_{4}\left(y, z, \mu_{I I}, h_{1}, h_{2}, h_{3}, h_{4}\right)=0
\end{aligned}
$$




$$
\begin{aligned}
& \frac{\partial h_{3}\left(y, z, \mu_{I I}\right)}{\partial y}\left[\lambda_{1} \cdot x+f_{1}\left(y, z, \mu_{I I}, h_{1}, h_{2}, h_{3}, h_{4}\right)\right]+ \\
& \quad+\frac{\partial h_{3}\left(y, z, \mu_{I I}\right)}{\partial z}\left[\lambda_{2} \cdot x+f_{2}\left(y, z, \mu_{I I}, h_{1}, h_{2}, h_{3}, h_{4}\right)\right]- \\
& \quad-\lambda_{4} \cdot h_{5}\left(y, z, \mu_{I I}\right)-f_{4}\left(y, z, \mu_{I I}, h_{1}, h_{2}, h_{3}, h_{4}\right)=0 \\
& \frac{\partial h_{4}\left(y, z, \mu_{I I}\right)}{\partial z}\left[\lambda_{2} \cdot x+f_{2}\left(y, z, \mu_{I I}, h_{1}, h_{2}, h_{3}, h_{4}\right)\right] \\
& \quad+\frac{\partial h_{4}\left(y, z, \mu_{I I}\right)}{\partial z}\left[\lambda_{2} \cdot x+f_{2}\left(y, z, \mu_{I I}, h_{1}, h_{2}, h_{3}, h_{4}\right)\right]- \\
& \quad-\lambda_{6} \cdot h_{4}\left(y, z, \mu_{I I}\right)-f_{6}\left(y, z, \mu_{I I}, h_{1}, h_{2}, h_{3}, h_{4}\right)=0
\end{aligned}
$$

Utilizando (5.58) e (5.60) em (5.61) e resolvendo o sistema de equações, é possível obter, igualando-se a zero os termos de igual potência, os coeficientes de $h_{1}\left(y, z, \mu_{I I}\right), h_{2}\left(y, z, \mu_{I I}\right), h_{3}\left(y, z, \mu_{I I}\right)$ e $h_{4}\left(y, z, \mu_{I I}\right)$ :

$$
\begin{aligned}
& y^{2}: \alpha_{1}=0, \beta_{1}=0, \gamma_{1}=0, \theta_{1}=0 \\
& z^{3}: \alpha_{2}=0, \beta_{2}=0, \gamma_{2}=0, \theta_{2}=0 \\
& \mu_{I I}^{2}: \alpha_{3}=0, \beta_{3}=0, \gamma_{3}=0, \theta_{3}=0 \\
& y \cdot z: \alpha_{4}=0, \beta_{4}=0, \gamma_{4}=0, \theta_{4}=0 \\
& y \cdot \mu_{I I}: \alpha_{5}=0, \beta_{5}=0, \gamma_{5}=0, \theta_{5}=0 \\
& z \cdot \mu_{I I}: \alpha_{6}=\frac{\sqrt{c_{I}^{2}-4 b_{I}}}{c_{I}^{2}-4 b_{I}} k, \beta_{6}=-\frac{\sqrt{c_{I}^{2}-4 b_{I}}}{c_{I}^{2}-4 b_{I}}, \gamma_{6}=0, \theta_{6}=0
\end{aligned}
$$

Com os coeficientes (5.62) dos polinômios de (5.60), pode-se obter, através do sistema (5.58), o campo de vetores restrito à variedade central, desprezando-se os termos de ordem superior:

$$
\left\{\begin{array}{l}
\dot{y}=-z \cdot \mu_{I I}\left[\left(a_{I I}-c_{I}\right) k \cdot \mu_{I I} \cdot y+y+a_{I I} \cdot k \cdot z \cdot \mu_{I I}\left(c_{I} \cdot k \cdot \mu_{I I}-1\right)\right] \\
\dot{z}=\mp k \cdot z \cdot \mu_{I I}\left[ \pm c_{I} \cdot k \cdot \mu_{I I}+a_{I}\left( \pm c_{I} \cdot z \cdot k \cdot \mu_{I I}+k \mp z\right) \mu_{I I} \mp 1\right]
\end{array}\right.
$$

Para obter os pontos de equilíbrio de (5.63), basta fazer:

$$
\left\{\begin{array}{l}
\dot{y}=0 \\
\dot{z}=0
\end{array}\right.
$$


De (5.64) se obtém os seguintes possíveis valores para y e $z$ :

- $y= \pm \frac{a_{I I} \cdot k\left(a_{I I} \cdot k \cdot \mu_{I I} \pm c_{I} \cdot k \cdot \mu_{I I} \mp 1\right)}{a_{I}\left(a_{I I} \cdot k \cdot \mu_{I I}-c_{I} \cdot k \cdot \mu_{I I}+1\right)}$ e $z=\frac{\mp a_{I} \cdot k \cdot \mu_{I I}-c_{I} \cdot k \cdot \mu_{I I}+1}{a_{I} \cdot \mu_{I I}\left(c_{I} \cdot k \cdot \mu_{I I}-1\right)}$ sendo $a_{I} \cdot \mu_{I I}\left(c_{I} \cdot k \cdot \mu_{I I}-1\right) \neq 0$ e $a_{I}\left(a_{I I} \cdot k \cdot \mu_{I I}-c_{I} \cdot k \cdot \mu_{I I}+1\right) \neq 0$

- $y= \pm \frac{a_{I I} \cdot k\left(a_{I I} \cdot k \cdot \mu_{I I} \pm c_{I} \cdot k \cdot \mu_{I I} \mp 1\right)}{a_{I}\left(a_{I I} \cdot k \cdot \mu_{I I}-c_{I} \cdot k \cdot \mu_{I I}+1\right)}$ e $z=0$, sendo $a_{I}\left(a_{I I} \cdot k \cdot \mu_{I I}-c_{I} \cdot k \cdot \mu_{I I}+1\right) \neq 0$

Para o estudo das estabilidades dos pontos de equilíbrio de (5.65), é preciso obter a matriz Jacobiana de (5.64):

$$
J_{P}=\left[\begin{array}{ll}
\left.\frac{\partial \dot{y}}{\partial y}\right|_{P} & \left.\frac{\partial \dot{y}}{\partial z}\right|_{P} \\
\left.\frac{\partial \dot{z}}{\partial y}\right|_{P} & \left.\frac{\partial \dot{z}}{\partial z}\right|_{P}
\end{array}\right]
$$

Sendo que em (5.67), tem-se:

$$
\begin{aligned}
& \frac{\partial \dot{y}}{\partial y}=z \cdot \mu_{I I}\left(\left(c_{I}-a_{I I}\right) k \cdot \mu_{I I}-1\right) \\
& \frac{\partial \dot{y}}{\partial z}=-\mu_{I I}\left(\left(a_{I I}-c_{I}\right) k \cdot \mu_{I I} \cdot y+y+2 a_{I I} \cdot k \cdot z \cdot \mu_{I I}\left(c_{I} \cdot k \cdot \mu_{I I}-1\right)\right) \\
& \frac{\partial \dot{z}}{\partial y}=0 \\
& \frac{\partial \dot{z}}{\partial z}=\mp k \cdot \mu_{I I}\left( \pm c_{I} \cdot k \cdot \mu_{I I}+a_{I}\left( \pm 2 c_{I} \cdot z \cdot k \cdot \mu_{I I}+k \mp 2 z\right) \mu_{I I} \mp 1\right)
\end{aligned}
$$

Em seguida, verifica-se a parte real dos autovalores para a matriz Jacobiana (5.66) calculada em cada ponto de equilíbrio:

- $y= \pm \frac{a_{I I} \cdot k\left(a_{I I} \cdot k \cdot \mu_{I I} \pm c_{I} \cdot k \cdot \mu_{I I} \mp 1\right)}{a_{I}\left(a_{I I} \cdot k \cdot \mu_{I I}-c_{I} \cdot k \cdot \mu_{I I}+1\right)}$ e $z=\frac{\mp a_{I} \cdot k \cdot \mu_{I I}-c_{I} \cdot k \cdot \mu_{I I}+1}{a_{I} \cdot \mu_{I I}\left(c_{I} \cdot k \cdot \mu_{I I}-1\right)}$, sendo $a_{I} \cdot \mu_{I I}\left(c_{I} \cdot k \cdot \mu_{I I}-1\right) \neq 0$ e $a_{I}\left(a_{I I} \cdot k \cdot \mu_{I I}-c_{I} \cdot k \cdot \mu_{I I}+1\right) \neq 0$ 


$$
\begin{aligned}
& \lambda_{1}=\frac{\left(\left(a_{I I}-c_{I}\right) k \cdot \mu_{I I}+1\right)\left(\left(c_{I} \pm a_{I}\right) k \cdot \mu_{I I}-1\right)}{a_{I}\left(c_{I} \cdot k \cdot \mu_{I I}-1\right)}, \\
& \lambda_{2}=k \cdot \mu_{I I}\left(\left(c_{I} \pm a_{I}\right) \cdot k \cdot \mu_{I I}-1\right) \\
& \text { Para P3: }\left(\operatorname{Re}\left(\lambda_{1}\right)<0 \vee \operatorname{Re}\left(\lambda_{2}\right)<0\right) \Leftrightarrow \\
& \left(\mu_{I I}<0 \wedge\left(\left(a_{I}<0 \wedge\left(\left(a_{I I} \leq 0 \wedge\left(\left(c_{I}<\frac{a_{I I} \cdot k \cdot \mu_{I I}+1}{k \cdot \mu_{I I}} \vee\right.\right.\right.\right.\right.\right.\right. \\
& \left.\left.\vee \frac{1}{k \cdot \mu_{I I}} c_{I}<\frac{1-a_{I I} \cdot k \cdot \mu_{I I}}{k \cdot \mu_{I I}}\right)\right) \vee\left(0<a_{I I}<-a_{I} \wedge\left(c_{I}<\frac{1}{k \cdot \mu_{I I}} \vee\right.\right. \\
& \left.\left.\vee \frac{a_{I I} \cdot k \cdot \mu_{I I}+1}{k \cdot \mu_{I I}}<c_{I}<\frac{1-a_{I I} \cdot k \cdot \mu_{I I}}{k \cdot \mu_{I I}}\right)\right) \vee\left(a_{I I} \geq-a_{I} \wedge\right. \\
& \left.\left.\left.\left.\left.\wedge c_{I}<\frac{1}{k \cdot \mu_{I I}}\right)\right)\right) \vee\left(a_{I}>0 \wedge a_{I I}<-a_{I} \wedge \frac{a_{I I} \cdot k \cdot \mu_{I I}+1}{k \cdot \mu_{I I}}<c_{I}<\frac{1-a_{I I} \cdot k \cdot \mu_{I I}}{k \cdot \mu_{I I}}\right)\right)\right) \vee \\
& \vee\left(\mu_{I I}>0 \wedge\left(\left(a_{I}<0 \wedge\left(\left(a_{I I}<0 \wedge \frac{a_{I I} \cdot k \cdot \mu_{I I}+1}{k \cdot \mu_{I I}}<c_{I}<\frac{1}{k \cdot \mu_{I I}}\right) \vee\right.\right.\right.\right. \\
& \vee\left(0<a_{I I} \leq-a_{I} \wedge \frac{1}{k \cdot \mu_{I I}}<c_{I}<\frac{a_{I I} \cdot k \cdot \mu_{I I}+1}{k \cdot \mu_{I I}}\right) \vee\left(a_{I I}>-a_{I} \wedge\right. \\
& \left.\left.\left.\wedge \frac{1}{k \cdot \mu_{I I}}<c_{I}<\frac{1-a_{I I} \cdot k \cdot \mu_{I I}}{k \cdot \mu_{I I}}\right)\right)\right) \vee \\
& \vee\left(a_{I}>0 \wedge\left(( a _ { I I } \leq - a _ { I } \wedge c _ { I } < \frac { a _ { I I } \cdot k \cdot \mu _ { I I } + 1 } { k \cdot \mu _ { I I } } ) \vee \left(a_{I I}>-a_{I} \wedge\right.\right.\right. \\
& \left.\left.\left.\left.\left.\left.\left.\wedge c_{I}<\frac{a_{I I} \cdot k \cdot \mu_{I I}+1}{k \cdot \mu_{I I}}\right)\right)\right)\right)\right)\right)\right) \vee\left(k>0 \wedge\left(\left(\mu_{I I}<0 \wedge\left(\left(a_{I}<0 \wedge\right.\right.\right.\right.\right. \\
& \vee\left(\left(a_{I I}<0 \wedge \frac{a_{I I} \cdot k \cdot \mu_{I I}+1}{k \cdot \mu_{I I}}<c_{I}<\frac{1}{k \cdot \mu_{I I}}\right) \vee\right. \\
& \vee\left(0<a_{I I} \leq-a_{I} \wedge \frac{1}{k \cdot \mu_{I I}}<c_{I}<\frac{a_{I I} \cdot k \cdot \mu_{I I}+1}{k \cdot \mu_{I I}}\right) \vee
\end{aligned}
$$

120 


$$
\begin{aligned}
& \left.\left.\vee\left(a_{I I}>-a_{I} \wedge \frac{1}{k \cdot \mu_{I I}}<c_{I}<\frac{1-a_{I I} \cdot k \cdot \mu_{I I}}{k \cdot \mu_{I I}}\right)\right)\right) \vee \\
& \left.\left.\vee\left(a_{I I}>-a_{I} \wedge c_{I}<\frac{1}{k \cdot \mu_{I I}}\right)\right)\right) \vee \\
& \left.\left.\left.\left.\vee\left(a_{I}>0 \wedge a_{I I}<-a_{I} \wedge \frac{a_{I I} \cdot k \cdot \mu_{I I}+1}{k \cdot \mu_{I I}}<c_{I}<\frac{1-a_{I I} \cdot k \cdot \mu_{I I}}{k \cdot \mu_{I I}}\right)\right)\right)\right)\right)
\end{aligned}
$$$$
\left(\operatorname{Re}\left(\lambda_{1}\right)>0 \vee \operatorname{Re}\left(\lambda_{2}\right)>0\right) \Leftrightarrow
$$$$
\left(\mu_{I I}<0 \wedge\left(( a _ { I } < 0 \wedge ) \left(\left(a_{I I}<0 \wedge\left(\left(\frac{a_{I I} \cdot k \cdot \mu_{I I}+1}{k \cdot \mu_{I I}}<c_{I}<\frac{1}{k \cdot \mu_{I I}} \vee\right.\right.\right.\right.\right.\right.
$$$$
\left.\left.\vee c_{I}<\frac{1-a_{I I} \cdot k \cdot \mu_{I I}}{k \cdot \mu_{I I}}\right)\right) \vee\left(a_{I I}=0 \wedge c_{I}>\frac{1}{k \cdot \mu_{I I}}\right) \vee
$$$$
\vee\left(0<a_{I I}<-a_{I} \wedge\left(\frac{1}{k \cdot \mu_{I I}}<c_{I}<\frac{a_{I I} \cdot k \cdot \mu_{I I}+1}{k \cdot \mu_{I I}} \vee c_{I}>\frac{1-a_{I I} \cdot k \cdot \mu_{I I}}{k \cdot \mu_{I I}}\right)\right) \vee
$$$$
\left.\left.\vee\left(a_{I I}>-a_{I} \wedge\left(\frac{1}{k \cdot \mu_{I I}}<c_{I}<\frac{a_{I I} \cdot k \cdot \mu_{I I}+1}{k \cdot \mu_{I I}} \vee c_{I}>\frac{1-a_{I I} \cdot k \cdot \mu_{I I}}{k \cdot \mu_{I I}}\right)\right)\right)\right) \vee
$$$$
\vee\left(a_{I}=0 \wedge c_{I}>\frac{1}{k \cdot \mu_{I I}}\right) \vee
$$$$
\vee\left(a_{I}>0 \wedge\left(\left(a_{I I}<-a_{I} \wedge\left(c_{I}<\frac{a_{I I} \cdot k \cdot \mu_{I I}+1}{k \cdot \mu_{I I}} \vee c_{I}>\frac{1-a_{I I} \cdot k \cdot \mu_{I I}}{k \cdot \mu_{I I}}\right)\right) \vee\right.\right.
$$$$
\vee\left(a_{I I}=-a_{I} \wedge\left(c_{I}<\frac{a_{I I} \cdot k \cdot \mu_{I I}+1}{k \cdot \mu_{I I}} \vee c_{I}>\frac{a_{I I} \cdot k \cdot \mu_{I I}+1}{k \cdot \mu_{I I}}\right)\right) \vee
$$$$
\left.\left.\left.\left.\vee\left(a_{I I}>-a_{I} \wedge\left(c_{I}<\frac{1-a_{I I} \cdot k \cdot \mu_{I I}}{k \cdot \mu_{I I}} \vee c_{I}>\frac{1-a_{I I} \cdot k \cdot \mu_{I I}}{k \cdot \mu_{I I}}\right)\right)\right)\right)\right)\right) \vee
$$$$
\vee\left(\mu_{I I}=0 \wedge a_{I}>0 \vee\left(\mu_{I I}>0 \wedge\left(\left(a_{I}<0 \wedge\left(\left(a_{I I} \leq 0 \wedge\right.\right.\right.\right.\right.\right.
$$ 


$$
\begin{aligned}
& \left.\wedge\left(c_{I}<\frac{a_{I I} \cdot k \cdot \mu_{I I}+1}{k \cdot \mu_{I I}} \vee \frac{1}{k \cdot \mu_{I I}}<c_{I}<\frac{1-a_{I I} \cdot k \cdot \mu_{I I}}{k \cdot \mu_{I I}} \vee c_{I}>\frac{1-a_{I I} \cdot k \cdot \mu_{I I}}{k \cdot \mu_{I I}}\right)\right) \vee \\
& \vee\left(0<a_{I I}<-a_{I} \wedge\left(c_{I}<\frac{1}{k \cdot \mu_{I I}} \vee \frac{a_{I I} \cdot k \cdot \mu_{I I}+1}{k \cdot \mu_{I I}}<c_{I}<\frac{1-a_{I I} \cdot k \cdot \mu_{I I}}{k \cdot \mu_{I I}} \vee\right.\right. \\
& \left.\left.\vee c_{I}>\frac{1-a_{I I} \cdot k \cdot \mu_{I I}}{k \cdot \mu_{I I}}\right)\right) \vee \vee\left(a_{I I}=-a_{I} \wedge\left(c_{I}<\frac{1}{k \cdot \mu_{I I}} \vee c_{I}>\frac{a_{I I} \cdot k \cdot \mu_{I I}+1}{k \cdot \mu_{I I}}\right)\right) \vee \\
& \left.\left.\vee\left(a_{I I}>-a_{I} \wedge\left(c_{I}<\frac{1}{k \cdot \mu_{I I}} \vee c_{I}>\frac{1}{k \cdot \mu_{I I}}\right)\right)\right)\right) \vee\left(a_{I}=0 \wedge c_{I}>\frac{1}{k \cdot \mu_{I I}}\right) \vee \\
& \vee\left(a_{I}>0 \wedge\left(a_{I I}<-a_{I} \wedge\left(\frac{a_{I I} \cdot k \cdot \mu_{I I}+1}{k \cdot \mu_{I I}}<c_{I}<\frac{1-a_{I I} \cdot k \cdot \mu_{I I}}{k \cdot \mu_{I I}} \vee\right.\right.\right. \\
& \left.\left.c_{I}>\frac{1-a_{I I} \cdot k \cdot \mu_{I I}}{k \cdot \mu_{I I}}\right)\right) \vee\left(a_{I I}=-a_{I} \wedge c_{I}>\frac{a_{I I} \cdot k \cdot \mu_{I I}+1}{k \cdot \mu_{I I}}\right) \vee \\
& \left.\left.\left.\left.\left.\left.\vee\left(a_{I I}>-a_{I} \wedge c_{I}>\frac{1-a_{I I} \cdot k \cdot \mu_{I I}}{k \cdot \mu_{I I}}\right)\right)\right)\right)\right)\right)\right) \vee \\
& \vee\left(k=0 \wedge a_{I}>0\right) \vee\left(k>0 \wedge\left(\left(\mu_{I I}<0 \wedge\left(\left(a_{I}<0 \wedge\left(\left(a_{I I} \leq 0 \wedge\right.\right.\right.\right.\right.\right.\right. \\
& \left.\vee\left(c_{I}<\frac{a_{I I} \cdot k \cdot \mu_{I I}+1}{k \cdot \mu_{I I}} \vee \frac{1}{k \cdot \mu_{I I}}<c_{I}<\frac{1-a_{I I} \cdot k \cdot \mu_{I I}}{k \cdot \mu_{I I}} \vee c_{I}>\frac{1-a_{I I} \cdot k \cdot \mu_{I I}}{k \cdot \mu_{I I}}\right)\right) \vee \\
& \vee\left(0<a_{I I}<-a_{I} \wedge\left(c_{I}<\frac{1}{k \cdot \mu_{I I}} \vee \frac{a_{I I} \cdot k \cdot \mu_{I I}+1}{k \cdot \mu_{I I}}<c_{I}<\frac{1-a_{I I} \cdot k \cdot \mu_{I I}}{k \cdot \mu_{I I}} \vee\right.\right. \\
& \left.\left.\vee c_{I}>\frac{1-a_{I I} \cdot k \cdot \mu_{I I}}{k \cdot \mu_{I I}}\right)\right) \vee\left(a_{I I}=-a_{I} \wedge\left(c_{I}<\frac{1}{k \cdot \mu_{I I}} \vee c_{I}>\frac{a_{I I} \cdot k \cdot \mu_{I I}+1}{k \cdot \mu_{I I}}\right)\right) \vee \\
& \left.\left.\vee\left(a_{I I}>-a_{I} \wedge\left(c_{I}<\frac{1}{k \cdot \mu_{I I}} \vee c_{I}>\frac{1-a_{I I} \cdot k \cdot \mu_{I I}}{k \cdot \mu_{I I}}\right)\right)\right)\right) \vee \\
& \vee\left(a_{I}=0 \wedge c_{I}>\frac{1}{k \cdot \mu_{I I}}\right) \vee \\
& \vee\left(a_{I}>0 \wedge\left(\left(a_{I I}<-a_{I} \wedge\left(\frac{a_{I I} \cdot k \cdot \mu_{I I}+1}{k \cdot \mu_{I I}}<c_{I}<\frac{1-a_{I I} \cdot k \cdot \mu_{I I}}{k \cdot \mu_{I I}} \vee\right.\right.\right.\right.
\end{aligned}
$$




$$
\begin{aligned}
& \left.\left.\vee c_{I}>\frac{1-a_{I I} \cdot k \cdot \mu_{I I}}{k \cdot \mu_{I I}}\right)\right) \vee\left(a_{I I}=-a_{I} \wedge c_{I}>\frac{a_{I I} \cdot k \cdot \mu_{I I}+1}{k \cdot \mu_{I I}}\right) \vee \\
& \left.\left.\left.\left.\vee\left(a_{I I}>-a_{I} \wedge c_{I}>\frac{1-a_{I I} \cdot k \cdot \mu_{I I}}{k \cdot \mu_{I I}}\right)\right)\right)\right)\right) \vee \\
& \vee\left(\mu_{I I}=0 \wedge a_{I}>0\right) \vee\left(\mu_{I I}>0 \wedge\left(\left(a_{I}<0 \wedge\left(\left(a_{I I} \leq 0 \wedge\right.\right.\right.\right.\right. \\
& \left.\vee\left(\frac{a_{I I} \cdot k \cdot \mu_{I I}+1}{k \cdot \mu_{I I}}<c_{I}<\frac{1}{k \cdot \mu_{I I}} \vee c_{I}>\frac{1-a_{I I} \cdot k \cdot \mu_{I I}}{k \cdot \mu_{I I}}\right)\right) \vee \\
& \vee\left(a_{I I}=0 \wedge c_{I}>\frac{1-a_{I I} \cdot k \cdot \mu_{I I}}{k \cdot \mu_{I I}}\right) \vee\left(0<a_{I I}<-a_{I} \wedge\right. \\
& \left.\vee\left(\frac{1}{k \cdot \mu_{I I}}<c_{I}<\frac{a_{I I} \cdot k \cdot \mu_{I I}+1}{k \cdot \mu_{I I}} \vee c_{I}>\frac{1-a_{I I} \cdot k \cdot \mu_{I I}}{k \cdot \mu_{I I}}\right)\right) \vee \\
& \vee\left(a_{I I}=-a_{I} \wedge\left(\frac{1}{k \cdot \mu_{I I}}<c_{I}<\frac{a_{I I} \cdot k \cdot \mu_{I I}+1}{k \cdot \mu_{I I}} \vee c_{I}>\frac{a_{I I} \cdot k \cdot \mu_{I I}+1}{k \cdot \mu_{I I}}\right)\right) \vee \\
& \left.\left.\vee\left(a_{I I}>-a_{I} \wedge\left(\frac{1}{k \cdot \mu_{I I}}<c_{I}<\frac{a_{I I} \cdot k \cdot \mu_{I I}+1}{k \cdot \mu_{I I}} \vee c_{I}>\frac{a_{I I} \cdot k \cdot \mu_{I I}+1}{k \cdot \mu_{I I}}\right)\right)\right)\right) \vee \\
& \vee\left(a_{I}=0 \wedge c_{I}>\frac{1}{k \cdot \mu_{I I}}\right) \vee \\
& \vee\left(a_{I}>0 \wedge\left(\left(a_{I I}<-a_{I} \wedge\left(c_{I}<\frac{a_{I I} \cdot k \cdot \mu_{I I}+1}{k \cdot \mu_{I I}} \vee c_{I}>\frac{1-a_{I I} \cdot k \cdot \mu_{I I}}{k \cdot \mu_{I I}}\right)\right) \vee\right.\right. \\
& \vee\left(a_{I I}=-a_{I} \wedge\left(c_{I}<\frac{a_{I I} \cdot k \cdot \mu_{I I}+1}{k \cdot \mu_{I I}} \vee c_{I}>\frac{a_{I I} \cdot k \cdot \mu_{I I}+1}{k \cdot \mu_{I I}}\right)\right) \vee \\
& \left.\left.\left.\left.\left.\left.\vee\left(a_{I I}>-a_{I} \wedge\left(c_{I}<\frac{1-a_{I I} \cdot k \cdot \mu_{I I}}{k \cdot \mu_{I I}} \vee c_{I}>\frac{1-a_{I I} \cdot k \cdot \mu_{I I}}{k \cdot \mu_{I I}}\right)\right)\right)\right)\right)\right)\right)\right)
\end{aligned}
$$

Para P4: $\left(\operatorname{Re}\left(\lambda_{1}\right)<0 \vee \operatorname{Re}\left(\lambda_{2}\right)<0\right) \Leftrightarrow$ 


$$
\begin{aligned}
& \left(k<0 \wedge\left(\left(\mu_{I I}<0 \wedge\left(\left(a_{I}<0 \wedge\left(\left(a_{I I} \leq a_{I} \wedge c_{I}<\frac{a_{I I} \cdot k \cdot \mu_{I I}+1}{k \cdot \mu_{I I}}\right) \vee\right.\right.\right.\right.\right.\right. \\
& \left.\left.\wedge\left(a_{I I}>a_{I} \wedge c_{I}<\frac{a_{I} \cdot k \cdot \mu_{I I}+1}{k \cdot \mu_{I I}}\right)\right)\right) \vee \\
& \vee\left(a_{I}>0 \wedge\left(\left(a_{I I}<0 \wedge \frac{a_{I I} \cdot k \cdot \mu_{I I}+1}{k \cdot \mu_{I I}}<c_{I}<\frac{1}{k \cdot \mu_{I I}}\right) \vee\right.\right. \\
& \vee\left(0<a_{I I} \leq a_{I} \wedge \frac{1}{k \cdot \mu_{I I}}<c_{I}<\frac{a_{I I} \cdot k \cdot \mu_{I I}+1}{k \cdot \mu_{I I}}\right) \vee\left(a_{I I}>a_{I} \wedge\right. \\
& \left.\left.\left.\left.\left.\wedge \frac{1}{k \cdot \mu_{I I}}<c_{I}<\frac{1+a_{I} \cdot k \cdot \mu_{I I}}{k \cdot \mu_{I I}}\right)\right)\right)\right) \text { }\right) \\
& \vee\left(\mu_{I I}>0 \wedge\left(\left(a_{I}<0 \wedge\left(\left(a_{I I}<a_{I} \wedge \frac{a_{I I} \cdot k \cdot \mu_{I I}+1}{k \cdot \mu_{I I}}<c_{I}<\frac{a_{I} \cdot k \cdot \mu_{I I}+1}{k \cdot \mu_{I I}}\right) \vee\right.\right.\right.\right. \\
& \vee\left(a_{I}>0 \wedge\left(\left(a_{I I} \leq 0 \wedge\left(\left(c_{I}<\frac{a_{I I} \cdot k \cdot \mu_{I I}+1}{k \cdot \mu_{I I}} \vee \frac{1}{k \cdot \mu_{I I}}<c_{I}<\frac{a_{I} \cdot k \cdot \mu_{I I}+1}{k \cdot \mu_{I I}}\right)\right) \vee\right.\right.\right. \\
& \vee\left(0<a_{I I} \leq a_{I} \wedge\left(c_{I}<\frac{1}{k \cdot \mu_{I I}} \vee \frac{a_{I I} \cdot k \cdot \mu_{I I}+1}{k \cdot \mu_{I I}}<c_{I}<\frac{a_{I} \cdot k \cdot \mu_{I I}+1}{k \cdot \mu_{I I}}\right)\right) \vee \\
& \left.\left.\left.\left.\left.\left.\vee\left(a_{I I} \geq a_{I} \wedge c_{I}<\frac{1}{k \cdot \mu_{I I}}\right)\right)\right)\right)\right)\right)\right) \vee \vee \\
& \vee\left(k>0 \wedge\left(\left(\mu_{I I}<0 \wedge\left(\left(a_{I}<0 \wedge\left(a_{I I}<a_{I} \wedge \vee\right.\right.\right.\right.\right.\right. \\
& \left.\wedge \frac{a_{I I} \cdot k \cdot \mu_{I I}+1}{k \cdot \mu_{I I}}<c_{I}<\frac{a_{I} \cdot k \cdot \mu_{I I}+1}{k \cdot \mu_{I I}}\right) \vee \\
& \vee\left(a_{I}>0 \wedge\left(a_{I I} \leq 0 \wedge\left(c_{I}<\frac{a_{I I} \cdot k \cdot \mu_{I I}+1}{k \cdot \mu_{I I}} \vee \frac{1}{k \cdot \mu_{I I}}<c_{I}<\frac{a_{I} \cdot k \cdot \mu_{I I}+1}{k \cdot \mu_{I I}}\right)\right) \vee\right. \\
& \vee\left(0<a_{I I} \leq a_{I} \wedge\left(c_{I}<\frac{1}{k \cdot \mu_{I I}} \vee \frac{a_{I I} \cdot k \cdot \mu_{I I}+1}{k \cdot \mu_{I I}}<c_{I}<\frac{a_{I} \cdot k \cdot \mu_{I I}+1}{k \cdot \mu_{I I}}\right)\right) \vee
\end{aligned}
$$

124 


$$
\begin{aligned}
& \left.\left.\left.\left.\left.\left.\vee\left(a_{I I} \geq a_{I} \wedge c_{I}<\frac{1}{k \cdot \mu_{I I}}\right)\right)\right)\right)\right)\right)\right) \vee \vee \\
& \vee\left(\mu_{I I}>0 \wedge\left(\left(a_{I}<0 \wedge\left(\left(a_{I I} \leq a_{I} \wedge c_{I}<\frac{a_{I I} \cdot k \cdot \mu_{I I}+1}{k \cdot \mu_{I I}}\right) \vee\right.\right.\right.\right. \\
& \left.\left.\vee\left(a_{I I}>a_{I} \wedge c_{I}<\frac{a_{I} \cdot k \cdot \mu_{I I}+1}{k \cdot \mu_{I I}}\right)\right)\right) \vee \\
& \vee\left(a_{I}>0 \wedge\left(a_{I I}<0 \wedge \frac{a_{I I} \cdot k \cdot \mu_{I I}+1}{k \cdot \mu_{I I}}<c_{I}<\frac{1}{k \cdot \mu_{I I}}\right) \vee\right. \\
& \vee\left(0<a_{I I}<a_{I} \wedge \frac{1}{k \cdot \mu_{I I}}<c_{I}<\frac{a_{I I} \cdot k \cdot \mu_{I I}+1}{k \cdot \mu_{I I}}\right) \vee \\
& \left.\left.\left.\left.\left.\vee\left(a_{I I}>a_{I} \wedge \frac{1}{k \cdot \mu_{I I}}<c_{I}<\frac{a_{I I} \cdot k \cdot \mu_{I I}+1}{k \cdot \mu_{I I}}\right)\right)\right)\right)\right)\right) \\
& \left(\operatorname{Re}\left(\lambda_{1}\right)>0 \vee \operatorname{Re}\left(\lambda_{2}\right)>0\right) \Leftrightarrow \\
& \left(k<0 \wedge\left(\left(\mu_{I I}<0 \wedge\left(\left(a_{I}<0 \wedge\left(\left(a_{I I}<a_{I} \wedge \vee\right.\right.\right.\right.\right.\right.\right. \\
& \left.\wedge\left(\frac{a_{I I} \cdot k \cdot \mu_{I I}+1}{k \cdot \mu_{I I}}<c_{I}<\frac{a_{I} \cdot k \cdot \mu_{I I}+1}{k \cdot \mu_{I I}} \vee c_{I}>\frac{a_{I I} \cdot k \cdot \mu_{I I}+1}{k \cdot \mu_{I I}}\right)\right) \vee \\
& \left.\left.\vee\left(a_{I I}=a_{I} \wedge c_{I}>\frac{a_{I I} \cdot k \cdot \mu_{I I}+1}{k \cdot \mu_{I I}}\right) \vee\left(a_{I I}>a_{I} \wedge c_{I}>\frac{a_{I} \cdot k \cdot \mu_{I I}+1}{k \cdot \mu_{I I}}\right)\right)\right) \vee \\
& \vee\left(a_{I}=0 \wedge c_{I}>\frac{1}{k \cdot \mu_{I I}}\right) \vee\left(a_{I}>0\left(\left(a_{I I} \leq 0 \wedge\left(c_{I}<\frac{a_{I I} \cdot k \cdot \mu_{I I}+1}{k \cdot \mu_{I I}} \vee\right.\right.\right.\right. \\
& \left.\left.\vee \frac{1}{k \cdot \mu_{I I}}<c_{I}<\frac{1+a_{I} \cdot k \cdot \mu_{I I}}{k \cdot \mu_{I I}} \vee c_{I}>\frac{1+a_{I} \cdot k \cdot \mu_{I I}}{k \cdot \mu_{I I}}\right)\right) \vee \\
& \vee\left(0<a_{I I}<a_{I} \wedge\left(c_{I}<\frac{1}{k \cdot \mu_{I I}} \vee \frac{a_{I I} \cdot k \cdot \mu_{I I}+1}{k \cdot \mu_{I I}}<c_{I}<\frac{a_{I} \cdot k \cdot \mu_{I I}+1}{k \cdot \mu_{I I}} \vee\right.\right.
\end{aligned}
$$

125 


$$
\begin{aligned}
& \left.\left.\vee c_{I}>\frac{1+a_{I} \cdot k \cdot \mu_{I I}}{k \cdot \mu_{I I}}\right)\right) \vee\left(a_{I I}=a_{I} \wedge\left(c_{I}<\frac{1}{k \cdot \mu_{I I}} \vee c_{I}>\frac{a_{I I} \cdot k \cdot \mu_{I I}+1}{k \cdot \mu_{I I}}\right)\right) \vee \\
& \left.\left.\left.\left.\vee\left(a_{I I}>a_{I} \wedge\left(c_{I}<\frac{1}{k \cdot \mu_{I I}} \vee c_{I}<\frac{a_{I} \cdot k \cdot \mu_{I I}+1}{k \cdot \mu_{I I}}\right)\right)\right)\right)\right)\right) \\
& \vee\left(\mu_{I I}=0 \wedge a_{I}>0\right) \vee\left(\mu_{I I}>0 \wedge\left(\left(a_{I}<0 \wedge\left(\left(a_{I I} \leq a_{I} \wedge\right.\right.\right.\right.\right. \\
& \left.\left.\wedge\left(c_{I}<\frac{a_{I I} \cdot k \cdot \mu_{I I}+1}{k \cdot \mu_{I I}} \vee c_{I}>\frac{1+a_{I} \cdot k \cdot \mu_{I I}}{k \cdot \mu_{I I}}\right)\right) \vee\left(a_{I I}=a_{I} \wedge c_{I} \neq \frac{a_{I I} \cdot k \cdot \mu_{I I}+1}{k \cdot \mu_{I I}}\right)\right) \vee \\
& \left.\left.\vee\left(a_{I I}>a_{I} \wedge\left(c_{I} \neq \frac{a_{I} \cdot k \cdot \mu_{I I}+1}{k \cdot \mu_{I I}}\right)\right)\right)\right) \vee \\
& \vee\left(a_{I}=0 \wedge c_{I}>\frac{1}{k \cdot \mu_{I I}}\right) \vee\left(a_{I}>0\left(\left(a_{I I} \leq 0 \wedge\left(\frac{a_{I I} \cdot k \cdot \mu_{I I}+1}{k \cdot \mu_{I I}}<c_{I} \frac{1}{k \cdot \mu_{I I}} \vee\right.\right.\right.\right. \\
& \left.\left.\wedge\left(c_{I}>\frac{1+a_{I} \cdot k \cdot \mu_{I I}}{k \cdot \mu_{I I}}\right)\right) \vee a_{I I}=0 \wedge c_{I}>\frac{a_{I} \cdot k \cdot \mu_{I I}+1}{k \cdot \mu_{I I}}\right) \vee \\
& \vee\left(0<a_{I I}<a_{I} \wedge\left(\frac{1}{k \cdot \mu_{I I}}<c_{I}<\frac{a_{I I} \cdot k \cdot \mu_{I I}+1}{k \cdot \mu_{I I}} \vee c_{I}>\frac{a_{I} \cdot k \cdot \mu_{I I}+1}{k \cdot \mu_{I I}}\right)\right) \vee \\
& \vee\left(a_{I I}=a_{I} \wedge\left(\frac{1}{k \cdot \mu_{I I}}<c_{I}<\frac{a_{I I} \cdot k \cdot \mu_{I I}+1}{k \cdot \mu_{I I}} \vee c_{I}>\frac{a_{I I} \cdot k \cdot \mu_{I I}+1}{k \cdot \mu_{I I}}\right)\right) \vee \\
& \left.\left.\left.\left.\left.\left.\left.\vee\left(a_{I I}>a_{I} \wedge\left(\frac{1}{k \cdot \mu_{I I}}<c_{I}<\frac{a_{I} \cdot k \cdot \mu_{I I}+1}{k \cdot \mu_{I I}} \vee c_{I}>\frac{a_{I} \cdot k \cdot \mu_{I I}+1}{k \cdot \mu_{I I}}\right)\right)\right)\right)\right)\right)\right)\right)\right) \vee \\
& \vee\left(k=0 \wedge a_{I}>0\right) \vee\left(k<0 \wedge\left(\left(\mu_{I I}<0 \wedge\left(a_{I I}<a_{I} \wedge c_{I} \neq \frac{a_{I} \cdot k \cdot \mu_{I I}+1}{k \cdot \mu_{I I}}\right) \vee\right.\right.\right. \\
& \left.\wedge\left(a_{I I}=a_{I} \wedge c_{I} \neq \frac{a_{I} \cdot k \cdot \mu_{I I}+1}{k \cdot \mu_{I I}}\right)\right) \vee \\
& \left.\left.\left.\vee\left(a_{I I}>a_{I} \wedge c_{I} \neq \frac{a_{I} \cdot k \cdot \mu_{I I}+1}{k \cdot \mu_{I I}}\right)\right)\right)\right) \vee\left(a_{I}=0 \wedge c_{I}>\frac{1}{k \cdot \mu_{I I}}\right) \vee
\end{aligned}
$$

126 


$$
\begin{aligned}
& \vee\left(a_{I}>0 \wedge\left(\left(a_{I}<0 \wedge\left(\frac{a_{I I} \cdot k \cdot \mu_{I I}+1}{k \cdot \mu_{I I}}<c_{I}<\frac{1}{k \cdot \mu_{I I}} \vee c_{I}>\frac{a_{I} \cdot k \cdot \mu_{I I}+1}{k \cdot \mu_{I I}}\right)\right) \vee\right.\right. \\
& \wedge\left(a_{I I}=0 \wedge c_{I}>\frac{a_{I} \cdot k \cdot \mu_{I I}+1}{k \cdot \mu_{I I}}\right) \vee\left(0<a_{I}<a_{I I} \wedge\right. \\
& \left.\wedge\left(\frac{1}{k \cdot \mu_{I I}}<c_{I}<\frac{a_{I I} \cdot k \cdot \mu_{I I}+1}{k \cdot \mu_{I I}} \vee c_{I}>\frac{a_{I} \cdot k \cdot \mu_{I I}+1}{k \cdot \mu_{I I}}\right)\right) \vee \\
& \wedge\left(a_{I I}=a_{I} \wedge\left(\frac{1}{k \cdot \mu_{I I}}<c_{I}<\frac{a_{I I} \cdot k \cdot \mu_{I I}+1}{k \cdot \mu_{I I}} \vee c_{I}>\frac{a_{I} \cdot k \cdot \mu_{I I}+1}{k \cdot \mu_{I I}}\right)\right) \vee \\
& \left.\left.\left.\left.\vee\left(a_{I I}>a_{I} \wedge\left(\frac{1}{k \cdot \mu_{I I}}<c_{I}<\frac{a_{I} \cdot k \cdot \mu_{I I}+1}{k \cdot \mu_{I I}} \vee c_{I}>\frac{a_{I} \cdot k \cdot \mu_{I I}+1}{k \cdot \mu_{I I}}\right)\right)\right)\right)\right)\right) \vee \\
& \vee\left(\mu_{I I}=0 \wedge a_{I}>0\right) \vee\left(\mu_{I I}>0 \wedge\left(a_{I}<0 \wedge\left(a_{I I}<a_{I} \wedge\right.\right.\right. \\
& \left.\wedge\left(\frac{a_{I I} \cdot k \cdot \mu_{I I}+1}{k \cdot \mu_{I I}}<c_{I}<\frac{a_{I} \cdot k \cdot \mu_{I I}+1}{k \cdot \mu_{I I}} \vee c_{I}>\frac{a_{I} \cdot k \cdot \mu_{I I}+1}{k \cdot \mu_{I I}}\right)\right) \vee \\
& \left.\left.\wedge\left(a_{I I}=a_{I} \wedge c_{I}>\frac{a_{I I} \cdot k \cdot \mu_{I I}+1}{k \cdot \mu_{I I}}\right) \vee\left(a_{I I}>a_{I} \wedge c_{I}>\frac{a_{I} \cdot k \cdot \mu_{I I}+1}{k \cdot \mu_{I I}}\right)\right)\right) \vee \\
& \wedge\left(a_{I}=0 \wedge c_{I}>\frac{1}{k \cdot \mu_{I I}}\right) \vee\left(a_{I}>0 \wedge\left(\left(a_{I I} \leq 0 \wedge c_{I}<\frac{a_{I I} \cdot k \cdot \mu_{I I}+1}{k \cdot \mu_{I I}} \vee\right.\right.\right. \\
& \left.\left.\vee \frac{1}{k \cdot \mu_{I I}}<c_{I}<\frac{a_{I I} \cdot k \cdot \mu_{I I}+1}{k \cdot \mu_{I I}} \vee c_{I}>\frac{a_{I} \cdot k \cdot \mu_{I I}+1}{k \cdot \mu_{I I}}\right)\right) \vee \\
& \vee\left(0<a_{I I}<a_{I} \wedge\left(c_{I}<\frac{1}{k \cdot \mu_{I I}} \vee \frac{a_{I I} \cdot k \cdot \mu_{I I}+1}{k \cdot \mu_{I I}}<c_{I}<\frac{a_{I} \cdot k \cdot \mu_{I I}+1}{k \cdot \mu_{I I}} \vee\right.\right. \\
& \left.\left.\vee c_{I}>\frac{a_{I} \cdot k \cdot \mu_{I I}+1}{k \cdot \mu_{I I}}\right)\right) \vee \\
& \vee\left(a_{I I}=a_{I} \wedge\left(c_{I}<\frac{1}{k \cdot \mu_{I I}} \vee c_{I}>\frac{a_{I I} \cdot k \cdot \mu_{I I}+1}{k \cdot \mu_{I I}}\right)\right) \vee \\
& \left.\left.\left.\left.\left.\left.\vee\left(a_{I I}>a_{I} \wedge\left(c_{I}<\frac{1}{k \cdot \mu_{I I}} \vee c_{I}>\frac{a_{I} \cdot k \cdot \mu_{I I}+1}{k \cdot \mu_{I I}}\right)\right)\right)\right)\right)\right)\right)\right)
\end{aligned}
$$


- $y= \pm \frac{a_{I I} \cdot k\left(a_{I I} \cdot k \cdot \mu_{I I} \pm c_{I} \cdot k \cdot \mu_{I I} \mp 1\right)}{a_{I}\left(a_{I I} \cdot k \cdot \mu_{I I}-c_{I} \cdot k \cdot \mu_{I I}+1\right)}$ e $z=0$, sendo

$$
a_{I}\left(a_{I I} \cdot k \cdot \mu_{I I}-c_{I} \cdot k \cdot \mu_{I I}+1\right) \neq 0
$$$$
\lambda_{1}=0, \lambda_{2}=\mp k \cdot \mu_{I I}\left(\left(a_{I} \pm c_{I}\right) \cdot k \cdot \mu_{I I} \mp 1\right)
$$$$
\left(\operatorname{Re}\left(\lambda_{2}\right)>0\right) \Leftrightarrow
$$

Para $\mathrm{P} 3, k \neq 0 \wedge \mu_{I I} \neq 0 \wedge c_{I}<\frac{1-a_{I I} \cdot k \cdot \mu_{I I}}{k \cdot \mu_{I I}}$ e para $\mathrm{P} 4$,

$$
k \neq 0 \wedge \mu_{I I} \neq 0 \wedge c_{I}<\frac{1+a_{I I} \cdot k \cdot \mu_{I I}}{k \cdot \mu_{I I}}
$$

A interpretação analítica e gráfica dos resultados apresentados será realizada no capítulo VI. A seguir, na próxima seção, será realizado um estudo equivalente para os pontos $P_{5,6}$.

\subsection{ESTUDO DOS PONTOS}

$$
P_{5,6}=\left(+\arccos \left[ \pm \sqrt{1-\left(\frac{\Omega}{\mu_{I}}\right)^{2}}\right], 0,0,+\arccos \left[ \pm \sqrt{1-\left(\frac{\Omega}{\mu_{I}}\right)^{2}}\right]+\arccos \left[ \pm \sqrt{1-\left(\frac{\Omega}{\mu_{I I}}\right)^{2}}\right], 0,0\right)
$$

\subsubsection{ANÁLISE LINEAR DA ESTABILIDADE}

Considere-se a seguinte expansão em série de Taylor das funções seno e co-

$$
\begin{aligned}
& \text { seno em torno } x_{1}^{*}=\arccos \left[ \pm \sqrt{1-\left(\frac{\Omega}{\mu_{I}}\right)^{2}}\right] \mathrm{e} \\
& x_{4}^{*}=+\arccos \left[ \pm \sqrt{1-\left(\frac{\Omega}{\mu_{I}}\right)^{2}}\right]+\arccos \left[ \pm \sqrt{1-\left(\frac{\Omega}{\mu_{I I}}\right)^{2}}\right]: \\
& \quad \operatorname{sen} x_{1} \approx+\operatorname{sen} \rho_{I}+\cos \rho_{I} \cdot\left(x_{1}-\rho_{I}\right)+\ldots, \cos x_{1} \approx+\cos \rho_{I}-\operatorname{sen} \rho_{I} \cdot\left(x_{1}-\rho_{I}\right)+\ldots \\
& \operatorname{sen} x_{4} \approx \operatorname{sen}\left(\rho_{I}+\rho_{I I}\right)+\ldots, \cos x_{4} \approx \cos \left(\rho_{I}+\rho_{I I}\right)+\ldots
\end{aligned}
$$


Em seguida, considere-se a seguinte mudança de coordenadas:

$$
\bar{x}_{1}=x_{1}-\left(x_{1}-\rho_{I}\right), \bar{x}_{2}=x_{2}, \bar{x}_{3}=x_{3}, \bar{x}_{4}=x_{4}, \bar{x}_{5}=x_{5} \text { e } \bar{x}_{6}=x_{6}
$$

Sendo que em (5.69) e (5.70), tem-se: $\rho_{I}=+\arccos \left[ \pm \sqrt{1-\left(\frac{\Omega}{\mu_{I}}\right)^{2}}\right]$ e

$$
\rho_{I I}=+\arccos \left[ \pm \sqrt{1-\left(\frac{\Omega}{\mu_{I I}}\right)^{2}}\right]
$$

A partir de (5.69), tem-se:

- $\cos \left(x_{4}-x_{1}\right)=\cos x_{4} \cos x_{1}+\operatorname{sen} x_{4} \operatorname{sen} x_{1}=\frac{\Omega}{\mu_{I I}} \cdot \bar{x}_{I} \pm \sqrt{1-\frac{\Omega^{2}}{\mu_{I I}^{2}}}$

- $\operatorname{sen}\left(x_{4}-x_{1}\right)=\operatorname{sen} x_{4} \cos x_{1}-\operatorname{sen} x_{1} \cos x_{4}=\frac{\Omega}{\mu_{I I}} \mp \sqrt{1-\frac{\Omega^{2}}{\mu_{I I}^{2}}} \cdot \bar{x}_{I}$

A seguir, considerando que $\mu_{I}=m \cdot \Omega,(m>1, m \in \mathbb{R})$, e que $\mu_{I I}=n \cdot \Omega$, $(n>1, n \in \mathbb{R})$, definem-se os seguintes parâmetros:

- $k_{I}=\sqrt{m^{2}-1}$, ou seja, $\Omega \cdot k_{I}=\sqrt{\mu_{I}^{2}-\Omega^{2}}$

- $k_{I I}=\sqrt{n^{2}-1}$, ou seja, $\Omega \cdot k_{I I}=\sqrt{\mu_{I I}^{2}-\Omega^{2}}$

Utilizando as expressões de (5.71), considerando que $\Omega<\mu_{I}, \Omega<\mu_{I I}$ e os parâmetros $k_{I}$ e $k_{I I}$ definidos em (5.53), reescreve-se o sistema (5.5): 


$$
\left\{\begin{array}{l}
\dot{\bar{x}}_{1}=\bar{x}_{2} \\
\dot{\bar{x}}_{2}=\bar{x}_{3} \\
\dot{\bar{x}}_{3}=-\frac{c_{I}}{b_{I}} \bar{x}_{3}-\frac{1}{b_{I}} \bar{x}_{2} \mp \frac{k_{I} \cdot \Omega \cdot a_{I}}{b_{I}} \bar{x}_{2}+\frac{a_{I} \cdot \Omega}{b_{I}} \bar{x}_{1} \cdot \bar{x}_{2} \mp \frac{\Omega \cdot k_{I}}{b_{I}} \bar{x}_{1} \\
\dot{\bar{x}}_{4}=x_{5} \\
\dot{\bar{x}}_{5}=x_{6} \\
\dot{\bar{x}}_{6}=-\frac{c_{I I}}{b_{I I}} \bar{x}_{6}-\frac{1}{b_{I I}} \bar{x}_{5} \pm \frac{k_{I I} \cdot \Omega}{b_{I I}} \bar{x}_{1}+\frac{a_{I I} \cdot \Omega}{b_{I I}} \bar{x}_{1} \cdot \bar{x}_{2}-\frac{a_{I I} \cdot \Omega}{b_{I I}} \bar{x}_{1} \cdot \bar{x}_{5} \pm \\
\pm \frac{a_{I I} \cdot k_{I I} \cdot \Omega}{b_{I I}} \bar{x}_{2} \mp \frac{a_{I I} \cdot k_{I I} \cdot \Omega}{b_{I I}} \bar{x}_{5}
\end{array}\right.
$$

Caso se transforme o parâmetro $\Omega$ em variável de estado, através da inclusão de uma equação diferencial sem dinâmica, ou seja, $\dot{\Omega}=0$, a matriz Jacobiana de (5.73), calculada nos pontos de equilíbrio $P_{3,4}=\left(\rho_{I}, 0,0, \rho_{I}+\rho_{I I}, 0,0\right)$, é:

$$
J_{P_{3,4}}=\left[\begin{array}{cccccc}
0 & 1 & 0 & 0 & 0 & 0 \\
0 & 0 & 1 & 0 & 0 & 0 \\
0 & -\frac{1}{b_{I}} & -\frac{c_{I}}{b_{I}} & 0 & 0 & 0 \\
0 & 0 & 0 & 0 & 1 & 0 \\
0 & 0 & 0 & 0 & 0 & 1 \\
0 & 0 & 0 & 0 & -\frac{1}{b_{I I}} & -\frac{c_{I I}}{b_{I I}}
\end{array}\right]
$$

A matriz (5.74) é idêntica à matriz (5.9). Dessa forma, todos os resultados pertinentes ao estudo da estabilidade do ponto $P$ podem ser aqui aplicados da mesma forma. Para proceder ao estudo da estabilidade, será aplicado o TVC.

\subsubsection{ANÁLISE VIA TVC}

O sistema (5.73) pode ser representado matricialmente como $\dot{\bar{X}}=J_{P} \cdot \bar{X}+\bar{F}$, ou seja: 


$$
\begin{aligned}
& {\left[\begin{array}{c}
\dot{\bar{x}}_{1} \\
\dot{\bar{x}}_{2} \\
\dot{\bar{x}}_{3} \\
\dot{\bar{x}}_{4} \\
\dot{\bar{x}}_{5} \\
\dot{\bar{x}}_{6}
\end{array}\right]=\left[\begin{array}{cccccc}
0 & 1 & 0 & 0 & 0 & 0 \\
0 & 0 & 1 & 0 & 0 & 0 \\
0 & -\frac{1}{b_{I}} & -\frac{c_{I}}{b_{I}} & 0 & 0 & 0 \\
0 & 0 & 0 & 0 & 1 & 0 \\
0 & 0 & 0 & 0 & 0 & 1 \\
0 & 0 & 0 & 0 & -\frac{1}{b_{I I}} & -\frac{c_{I I}}{b_{I I}}
\end{array}\right] \cdot\left[\begin{array}{l}
\bar{x}_{1} \\
\bar{x}_{2} \\
\bar{x}_{3} \\
\bar{x}_{4} \\
\bar{x}_{5} \\
\bar{x}_{6}
\end{array}\right]+} \\
& +\left[\begin{array}{c}
\mp \frac{k_{I} \cdot \Omega \cdot a_{I}}{b_{I}} \bar{x}_{2}+\frac{a_{I} \cdot \Omega}{b_{I}} \bar{x}_{1} \cdot \bar{x}_{2} \mp \frac{\Omega \cdot k_{I}}{b_{I}} \bar{x}_{1} \\
0 \\
0 \\
\left(\begin{array}{c} 
\pm \frac{k_{I I} \cdot \Omega}{b_{I I}} \bar{x}_{1}+\frac{a_{I I} \cdot \Omega}{b_{I I}} \bar{x}_{1} \cdot \bar{x}_{2}- \\
-\frac{a_{I I} \cdot \Omega}{b_{I I}} \bar{x}_{1} \cdot \bar{x}_{5} \pm \frac{a_{I I} \cdot k_{I I} \cdot \Omega}{b_{I I}} \bar{x}_{2} \mp \frac{a_{I I} \cdot k_{I I} \cdot \Omega}{b_{I I}} \bar{x}_{5}
\end{array}\right]
\end{array}\right.
\end{aligned}
$$

Conforme requerido pelo TVC, o sistema (5.54) precisa ser reescrito na forma canônica de Jordan. Para isso, define-se um novo conjunto de variáveis de estado $V=\left(v_{1}, v_{2}, v_{3}, v_{4}, v_{5}, v_{6}\right)^{T}$ de modo que $\bar{X}=M \cdot V$, sendo $M$ uma base composta pelos autovetores de $J_{P}$ de (5.10). Assim, é possível se estabelecer a seguinte relação entre as variáveis de estado $X$ e $V$ :

$$
\begin{aligned}
& \bar{x}_{1}=v_{2}+\frac{1}{2}\left(c_{I} \cdot \delta_{I}-2 b_{I}\right) v_{3}+\frac{1}{2}\left(c_{I} \cdot \varepsilon_{I}-2 b_{I}\right) v_{4} \\
& \bar{x}_{2}=\frac{1}{2} \delta_{I} \cdot v_{3}-\frac{1}{2} \varepsilon_{I} \cdot v_{4} \\
& \bar{x}_{3}=v_{3}+v_{4} \\
& \bar{x}_{4}=v_{1}+\frac{1}{2}\left(c_{I I} \cdot \delta_{I I}-2 b_{I I}\right) v_{5}+\frac{1}{2}\left(c_{I I} \cdot \varepsilon_{I I}-2 b_{I I}\right) v_{6} \\
& \bar{x}_{5}=\frac{1}{2} \delta_{I I} \cdot v_{5}-\frac{1}{2} \varepsilon_{I I} \cdot v_{6}
\end{aligned}
$$


$\bar{x}_{6}=v_{5}+v_{6}$

Sendo que em (5.76), tem-se $\delta=c-\sqrt{c^{2}-4 b}$ e $\varepsilon=c+\sqrt{c^{2}-4 b}$.

Dessa forma, é possível fazer $V=\left[M^{-1} \cdot J_{P} \cdot M\right] \cdot V+M^{-1} \cdot \bar{F} \quad$ e $\dot{\Omega}=0$, ou seja:

$$
\left[\begin{array}{l}
\dot{v}_{1} \\
\dot{v}_{2} \\
\dot{v}_{3} \\
\dot{v}_{4} \\
\dot{v}_{5} \\
\dot{v}_{6}
\end{array}\right]=\left[\begin{array}{cccccc}
\lambda_{1} & 0 & 0 & 0 & 0 & 0 \\
0 & \lambda_{2} & 0 & 0 & 0 & 0 \\
0 & 0 & \lambda_{3} & 0 & 0 & 0 \\
0 & 0 & 0 & \lambda_{4} & 0 & 0 \\
0 & 0 & 0 & 0 & \lambda_{5} & 0 \\
0 & 0 & 0 & 0 & 0 & \lambda_{6}
\end{array}\right] \cdot\left[\begin{array}{c}
v_{1} \\
v_{2} \\
v_{3} \\
v_{4} \\
v_{5} \\
v_{6}
\end{array}\right]+\left[\begin{array}{l}
f_{1} \\
f_{2} \\
f_{3} \\
f_{4} \\
f_{5} \\
f_{6}
\end{array}\right] \text { e } \dot{\Omega}=0
$$

Em (5.77), tem-se que:

- $\lambda_{1}=0$

- $\lambda_{2}=0$

- $\lambda_{3}=-\frac{\left(c_{I}+\sqrt{c_{I}^{2}-4 b_{I}}\right)}{2 b_{I}}$

- $\lambda_{4}=\frac{\left(\sqrt{c_{I}^{2}-4 b_{I}}-c_{I}\right)}{2 b_{I}}$

- $\lambda_{5}=-\frac{\left(c_{I I}+\sqrt{c_{I I}^{2}-4 b_{I I}}\right)}{2 b_{I I}}$

- $\lambda_{6}=\frac{\left(\sqrt{c_{I I}^{2}-4 b_{I I}}-c_{I I}\right)}{2 b_{I I}}$

- $\left[\begin{array}{l}f_{1} \\ f_{2} \\ f_{3} \\ f_{4} \\ f_{5} \\ f_{6}\end{array}\right]=M^{-1} \cdot \bar{F}$ 
Por questão de simplicidade, optou-se por omitir os termos não-lineares $f_{1}$, $f_{2}, f_{3}, f_{4}, f_{5}$ e $f_{6}$ devido a sua extensão.

Então, de acordo com o Teorema da Variedade Central (Guckenheimer e Holmes, 1983; Wiggins, 1990; Monteiro, 2002), procura-se a variedade central $W^{C}$ que seja tangente subespaço central $E^{C}$ no ponto de equilíbrio $P$. Assim, para $y, z$ e $\Omega$ suficientemente pequenos, tem-se:

$$
\begin{aligned}
W^{c}(0)=\{ & \left(v_{1}, v_{2}, v_{3}, v_{4}, v_{5}, v_{6}\right) \in \Re^{4} / v_{1}=y, v_{2}=z, \\
& v_{3}=h_{1}(y, z, \Omega), v_{4}=h_{2}(y, z, \Omega), \\
& v_{5}=h_{3}(y, z, \Omega), v_{6}=h_{4}(y, z, \Omega), \\
& \left.h_{j}(0,0,0)=0, D h_{j}(0,0,0)=0, j=1,2,3,4\right\}
\end{aligned}
$$

Considere-se então, os seguintes polinômios estimadores compostos por monômios do segundo grau $h_{1}(y, z, \Omega), h_{2}(y, z, \Omega), h_{3}(y, z, \Omega)$ e $h_{4}(y, z, \Omega)$ :

$$
\begin{aligned}
& h_{1}(y, z, \Omega)=\alpha_{1} \cdot y^{2}+\alpha_{2} \cdot z^{2}+\alpha_{3} \cdot \Omega^{2}+\alpha_{4} \cdot y \cdot z+\alpha_{5} \cdot y \cdot \Omega \\
& \quad+\alpha_{6} \cdot z \cdot \Omega,\left(\alpha_{j} \in \mathbb{R}, j=1, \ldots, 7\right) \\
& h_{2}(y, z, \Omega)=\beta_{1} \cdot y^{2}+\beta_{2} \cdot z^{2}+\beta_{3} \cdot \Omega^{2}+\beta_{4} \cdot y \cdot z+\beta_{5} \cdot y \cdot \Omega \\
& \quad+\beta_{6} \cdot z \cdot \Omega,\left(\beta_{j} \in \mathbb{R}, j=1, \ldots, 7\right) \\
& h_{3}(y, z, \Omega)=\gamma_{1} \cdot y^{2}+\gamma_{2} \cdot z^{2}+\gamma_{3} \cdot \Omega^{2}+\gamma_{4} \cdot y \cdot z+\gamma_{5} \cdot y \cdot \Omega \\
& \quad+\gamma_{6} \cdot z \cdot \Omega,\left(\gamma_{j} \in \mathbb{R}, j=1, \ldots, 7\right) \\
& h_{3}(y, z, \Omega)=\theta_{1} \cdot y^{2}+\theta_{2} \cdot z^{2}+\theta_{3} \cdot \Omega^{2}+\theta_{4} \cdot y \cdot z+\theta_{5} \cdot y \cdot \Omega \\
& \quad+\theta_{6} \cdot z \cdot \Omega,\left(\theta_{j} \in \mathbb{R}, j=1, \ldots, 7\right)
\end{aligned}
$$

Pelo teorema de Carr (Carr, 1981; Guckenheimer e Holmes, 1983; Wiggins, 1990), a variedade central $W^{c}$ deve satisfazer $N(h(y, z, \Omega))=0$. Portanto, tem-se:

$$
\begin{aligned}
& \frac{\partial h_{1}(y, z, \Omega)}{\partial y}\left[\lambda_{1} \cdot x+f_{1}\left(y, z, \Omega, h_{1}, h_{2}, h_{3}, h_{4}\right)\right]+ \\
& \quad+\frac{\partial h_{1}(y, z, \Omega)}{\partial z}\left[\lambda_{2} \cdot x+f_{2}\left(y, z, \Omega, h_{1}, h_{2}, h_{3}, h_{4}\right)\right]- \\
& \quad-\lambda_{3} \cdot h_{1}(y, z, \Omega)-f_{3}\left(y, z, \Omega, h_{1}, h_{2}, h_{3}, h_{4}\right)=0
\end{aligned}
$$




$$
\begin{aligned}
& \frac{\partial h_{2}(y, z, \Omega)}{\partial z}\left[\lambda_{2} \cdot x+f_{2}\left(y, z, \Omega, h_{1}, h_{2}, h_{3}, h_{4}\right)\right]+ \\
& +\frac{\partial h_{2}(y, z, \Omega)}{\partial z}\left[\lambda_{2} \cdot x+f_{2}\left(y, z, \Omega, h_{1}, h_{2}, h_{3}, h_{4}\right)\right]- \\
& \quad-\lambda_{4} \cdot h_{2}(y, z, \Omega)-f_{4}\left(y, z, \Omega, h_{1}, h_{2}, h_{3}, h_{4}\right)=0 \\
& \frac{\partial h_{3}(y, z, \Omega)}{\partial y}\left[\lambda_{1} \cdot x+f_{1}\left(y, z, \Omega, h_{1}, h_{2}, h_{3}, h_{4}\right)\right]+ \\
& \quad+\frac{\partial h_{3}(y, z, \Omega)}{\partial z}\left[\lambda_{2} \cdot x+f_{2}\left(y, z, \Omega, h_{1}, h_{2}, h_{3}, h_{4}\right)\right]- \\
& \quad-\lambda_{4} \cdot h_{5}(y, z, \Omega)-f_{4}\left(y, z, \Omega, h_{1}, h_{2}, h_{3}, h_{4}\right)=0 \\
& \frac{\partial h_{4}(y, z, \Omega)}{\partial z}\left[\lambda_{2} \cdot x+f_{2}\left(y, z, \Omega, h_{1}, h_{2}, h_{3}, h_{4}\right)\right] \\
& +\frac{\partial h_{4}(y, z, \Omega)}{\partial z}\left[\lambda_{2} \cdot x+f_{2}\left(y, z, \Omega, h_{1}, h_{2}, h_{3}, h_{4}\right)\right]- \\
& \quad-\lambda_{6} \cdot h_{4}(y, z, \Omega)-f_{6}\left(y, z, \Omega, h_{1}, h_{2}, h_{3}, h_{4}\right)=0
\end{aligned}
$$

Utilizando (5.77) e (5.79) em (5.80) e resolvendo o sistema de equações, é possível obter, igualando-se a zero os termos de igual potência, os coeficientes de

$$
\begin{aligned}
& h_{1}(y, z, \Omega), h_{2}(y, z, \Omega), h_{3}(y, z, \Omega) \text { e } h_{4}(y, z, \Omega): \\
& y^{2}: \alpha_{1}=0, \beta_{1}=0, \gamma_{1}=0, \theta_{1}=0 \\
& z^{3}: \alpha_{2}=0, \beta_{2}=0, \gamma_{2}=0, \theta_{2}=0 \\
& \Omega^{2}: \alpha_{3}=0, \beta_{3}=0, \gamma_{3}=0, \theta_{3}=0 \\
& y \cdot z: \alpha_{4}=0, \beta_{4}=0, \gamma_{4}=0, \theta_{4}=0 \\
& y \cdot \Omega: \alpha_{5}=\mp \frac{\sqrt{c_{I}^{2}-4 b_{I}}}{c_{I}^{2}-4 b_{I}} k_{I}, \beta_{5}= \pm \frac{\sqrt{c_{I}^{2}-4 b_{I}}}{c_{I}^{2}-4 b_{I}} k_{I}, \\
& \gamma_{5}= \pm \frac{\sqrt{c_{I I}^{2}-4 b_{I I}}}{c_{I I}^{2}-4 b_{I I}} k_{I I}, \theta_{5}=\mp \frac{\sqrt{c_{I I}^{2}-4 b_{I I}}}{c_{I I}^{2}-4 b_{I I}} k_{I I} \\
& z \cdot \Omega: \alpha_{6}=0, \beta_{6}=0, \gamma_{6}=0, \theta_{6}=0
\end{aligned}
$$


Com os coeficientes (5.81) dos polinômios de (5.80), pode-se obter, através do sistema (5.77), o campo de vetores restrito à variedade central, desprezando-se os termos de ordem superior:

$$
\left\{\begin{aligned}
\dot{y}= & -z \cdot \Omega\left[a_{I I} \cdot \Omega \cdot k_{I I}^{2}+\left(-c_{I} \cdot k_{I} \cdot \Omega+a_{I I}\left(c_{I} \cdot z \cdot \Omega \cdot k_{I} \pm k_{I} \pm z\right) \Omega \mp 1\right) k_{I I}+\right. \\
& \left.+a_{I I} \cdot k_{I} \cdot z \cdot \Omega\left(c_{I} \cdot k_{I} \cdot \Omega \pm 1\right)\right] \\
\dot{z}=\mp & k_{I} \cdot z \cdot \Omega\left[ \pm c_{I} \cdot k_{I} \cdot \Omega+a_{I}\left(z+k_{I}\left( \pm c_{I} \cdot z \cdot \Omega \mp 1\right)\right) \Omega+1\right]
\end{aligned}\right.
$$

Para obter os pontos de equilíbrio de (5.82), basta fazer:

$$
\left\{\begin{array}{l}
\dot{y}=0 \\
\dot{z}=0
\end{array}\right.
$$

De (5.83) se obtém:

$$
y=r, \forall r \in \mathbb{R} \text { e } z=0
$$

Para o estudo da estabilidade do ponto de equilíbrio de (5.84), é preciso obter a matriz Jacobiana de (5.82):

$$
J_{P}=\left[\begin{array}{ll}
\left.\frac{\partial \dot{y}}{\partial y}\right|_{P} & \left.\frac{\partial \dot{y}}{\partial z}\right|_{P} \\
\left.\frac{\partial \dot{z}}{\partial y}\right|_{P} & \left.\frac{\partial \dot{z}}{\partial z}\right|_{P}
\end{array}\right]
$$

Sendo que em (5.85), tem-se:

$$
\begin{aligned}
\frac{\partial \dot{y}}{\partial y}= & \\
\frac{\partial \dot{y}}{\partial z}= & -\Omega\left[a_{I I} \cdot \Omega \cdot k_{I I}^{2}+\left(-c_{I} \cdot k_{I} \cdot \Omega+a_{I I}\left(2 c_{I} \cdot z \cdot \Omega \cdot k_{I}+k_{I}+2 z\right) \Omega-1\right) k_{I I}\right. \\
& \left.+2 a_{I I} \cdot k_{I} \cdot z \cdot \Omega\left(c_{I} \cdot k_{I} \cdot \Omega+1\right)\right] \\
\frac{\partial \dot{z}}{\partial y}= & 0 \\
\frac{\partial \dot{z}}{\partial z}= & \mp k_{I} \cdot \Omega\left[ \pm c_{I} \cdot k_{I} \cdot \Omega+a_{I}\left(2 z+k_{I}\left( \pm 2 c_{I} \cdot z \cdot \Omega \mp 1\right)\right) \Omega+1\right]
\end{aligned}
$$

Em seguida, verifica-se a parte real dos autovalores para a matriz Jacobiana (5.85) calculada no ponto de equilíbrio: 


$$
\begin{aligned}
& \lambda_{1}=0, \lambda_{2}=\mp k_{I} \cdot \Omega\left[ \pm c_{I} \cdot k_{I} \cdot \Omega+a_{I}\left(-2 c_{I} \cdot z \cdot \Omega \cdot k_{I}+k_{I}+2 z\right) \Omega+1\right] \\
& \left(\operatorname{Re}\left(\lambda_{2}\right)>0\right) \Leftrightarrow \operatorname{Para} \mathrm{P} 5, \\
& \left(c_{I}<a_{I} \wedge\left(\left(k_{I}<0 \wedge\left(\Omega<\frac{1}{a_{I} \cdot k_{I}-c_{I} \cdot k_{I}} \vee \Omega>0\right)\right) \vee\right.\right. \\
& \left.\left.\vee\left(k_{I}>0 \wedge\left(\Omega<0 \vee \Omega>\frac{1}{a_{I} \cdot k_{I}-c_{I} \cdot k_{I}}\right)\right)\right)\right) \vee \\
& \vee\left(c_{I}=a_{I} \wedge\left(\left(k_{I}<0 \wedge \Omega>0\right) \vee\left(k_{I}>0 \wedge \Omega<0\right)\right)\right) \vee \\
& \vee\left(c_{I}>a_{I} \wedge\left(\left(k_{I}<0 \wedge 0<\Omega<\frac{1}{a_{I} \cdot k_{I}-c_{I} \cdot k_{I}}\right) \vee\right.\right. \\
& \left.\left.\vee\left(k_{I}>0 \wedge \frac{1}{a_{I} \cdot k_{I}-c_{I} \cdot k_{I}}<\Omega<0\right)\right)\right)
\end{aligned}
$$

E para P6,

$$
\begin{aligned}
& \text { Para P5, }\left(c_{I}<a_{I} \wedge\left(\left(k_{I}<0 \wedge\left(\Omega>-\frac{1}{a_{I} \cdot k_{I}-c_{I} \cdot k_{I}} \vee \Omega<0\right)\right) \vee\right.\right. \\
& \left.\left.\vee\left(k_{I}>0 \wedge\left(\Omega>0 \vee \Omega<-\frac{1}{a_{I} \cdot k_{I}-c_{I} \cdot k_{I}}\right)\right)\right)\right) \vee \\
& \vee\left(c_{I}=a_{I} \wedge\left(\left(k_{I}<0 \wedge \Omega<0\right) \vee\left(k_{I}>0 \wedge \Omega>0\right)\right)\right) \vee \\
& \vee\left(c_{I}>a_{I} \wedge\left(\left(k_{I}<0 \wedge-\frac{1}{a_{I} \cdot k_{I}-c_{I} \cdot k_{I}}<\Omega<0\right) \vee\right.\right. \\
& \left.\left.\vee\left(k_{I}>0 \wedge 0<\Omega<-\frac{1}{a_{I} \cdot k_{I}-c_{I} \cdot k_{I}}\right)\right)\right)
\end{aligned}
$$

A interpretação analítica e gráfica dos resultados apresentados será realizada no capítulo VI. A seguir, na próxima seção, será realizado um estudo equivalente para os pontos $P_{7,8}$. 


\subsection{ESTUDO DOS PONTOS}

$$
P_{7,8}=\left(+\arccos \left[ \pm \sqrt{1-\left(\frac{\Omega}{\mu_{I}}\right)^{2}}\right], 0,0,+\arccos \left[ \pm \sqrt{1-\left(\frac{\Omega}{\mu_{I}}\right)^{2}}\right]+\arccos \left[\mp \sqrt{1-\left(\frac{\Omega}{\mu_{I I}}\right)^{2}}\right], 0,0\right)
$$

\subsubsection{ANÁLISE LINEAR DA ESTABILIDADE}

Considere-se a seguinte expansão em série de Taylor das funções seno e co-

$$
\begin{aligned}
& \text { seno em torno } x_{1}^{*}=\arccos \left[ \pm \sqrt{1-\left(\frac{\Omega}{\mu_{I}}\right)^{2}}\right] \mathrm{e} \\
& x_{4}^{*}=+\arccos \left[ \pm \sqrt{1-\left(\frac{\Omega}{\mu_{I}}\right)^{2}}\right]+\arccos \left[\mp \sqrt{1-\left(\frac{\Omega}{\mu_{I I}}\right)^{2}}\right]: \\
& \operatorname{sen} x_{1} \approx+\operatorname{sen} \rho_{I}+\cos \rho_{I} \cdot\left(x_{1}-\rho_{I}\right)+\ldots, \cos x_{1} \approx+\cos \rho_{I}-\operatorname{sen} \rho_{I} \cdot\left(x_{1}-\rho_{I}\right)+\ldots \\
& \operatorname{sen} x_{4} \approx \operatorname{sen}\left(\rho_{I}+\rho_{I I}\right)+\ldots, \cos x_{4} \approx \cos \left(\rho_{I}+\rho_{I I}\right)+\ldots
\end{aligned}
$$

Sendo que em (5.87), tem-se: $\rho_{I}=+\arccos \left[ \pm \sqrt{1-\left(\frac{\Omega}{\mu_{I}}\right)^{2}}\right] \mathrm{e}$

$$
\rho_{I I}=+\arccos \left[\mp \sqrt{1-\left(\frac{\Omega}{\mu_{I I}}\right)^{2}}\right]
$$

Em seguida, considere-se a seguinte mudança de coordenadas:

$$
\bar{x}_{1}=x_{1}-\rho_{I}, \bar{x}_{2}=x_{2}, \bar{x}_{3}=x_{3}, \bar{x}_{4}=x_{4}, \bar{x}_{5}=x_{5} \text { e } \bar{x}_{6}=x_{6}
$$

A partir de (5.87) e (5.88), tem-se:

- $\cos \left(x_{4}-x_{1}\right)=\cos x_{4} \cos x_{1}+\operatorname{sen} x_{4} \operatorname{sen} x_{1}=\frac{\Omega}{\mu_{I I}} \cdot \bar{x}_{I} \mp \sqrt{1-\frac{\Omega^{2}}{\mu_{I I}^{2}}}$

- $\operatorname{sen}\left(x_{4}-x_{1}\right)=\operatorname{sen} x_{4} \cos x_{1}-\operatorname{sen} x_{1} \cos x_{4}=\frac{\Omega}{\mu_{I I}} \pm \sqrt{1-\frac{\Omega^{2}}{\mu_{I I}^{2}}} \cdot \bar{x}_{I}$ 
A seguir, considerando que $\mu_{I}=m \cdot \Omega,(m>1, m \in \mathbb{R})$, e que $\mu_{I I}=n \cdot \Omega$, $(n>1, n \in \mathbb{R})$, definem-se os seguintes parâmetros:

- $k_{I}=\sqrt{m^{2}-1}$, ou seja, $\Omega \cdot k_{I}=\sqrt{\mu_{I}^{2}-\Omega^{2}}$

- $k_{I I}=\sqrt{n^{2}-1}$, ou seja, $\Omega \cdot k_{I I}=\sqrt{\mu_{I I}^{2}-\Omega^{2}}$

Utilizando as expressões de (5.89), considerando que $\Omega<\mu_{I}, \Omega<\mu_{I I}$ e os parâmetros $k_{I}$ e $k_{I I}$ definidos em (5.90), reescreve-se o sistema (5.5), já transladado para a origem:

$$
\left\{\begin{array}{l}
\dot{\bar{x}}_{1}=\bar{x}_{2} \\
\dot{\bar{x}}_{2}=\bar{x}_{3} \\
\dot{\bar{x}}_{3}=-\frac{c_{I}}{b_{I}} \bar{x}_{3}-\frac{1}{b_{I}} \bar{x}_{2} \pm \frac{k_{I} \cdot \Omega \cdot a_{I}}{b_{I}} \bar{x}_{2}+\frac{a_{I} \cdot \Omega}{b_{I}} \bar{x}_{1} \cdot \bar{x}_{2} \mp \frac{\Omega \cdot k_{I}}{b_{I}} \bar{x}_{1} \\
\dot{\bar{x}}_{4}=x_{5} \\
\dot{\bar{x}}_{5}=x_{6} \\
\dot{\bar{x}}_{6}=-\frac{c_{I I}}{b_{I I}} \bar{x}_{6}-\frac{1}{b_{I I}} \bar{x}_{5} \mp \frac{k_{I I} \cdot \Omega}{b_{I I}} \bar{x}_{1}+\frac{a_{I I} \cdot \Omega}{b_{I I}} \bar{x}_{1} \cdot \bar{x}_{2}-\frac{a_{I I} \cdot \Omega}{b_{I I}} \bar{x}_{1} \cdot \bar{x}_{5} \mp \\
\mp \frac{a_{I I} \cdot k_{I I} \cdot \Omega}{b_{I I}} \bar{x}_{2} \pm \frac{a_{I I} \cdot k_{I I} \cdot \Omega}{b_{I I}} \bar{x}_{5}
\end{array}\right.
$$

Caso se transforme o parâmetro $\Omega$ em variável de estado, através da inclusão de uma equação diferencial sem dinâmica, ou seja, $\dot{\Omega}=0$, a matriz Jacobiana de (5.73), calculada nos pontos de equilíbrio $P_{7,8}=\left(\rho_{I}, 0,0, \rho_{I}+\rho_{I I}, 0,0\right)$, é: 


$$
J_{P_{3,4}}=\left[\begin{array}{cccccc}
0 & 1 & 0 & 0 & 0 & 0 \\
0 & 0 & 1 & 0 & 0 & 0 \\
0 & -\frac{1}{b_{I}} & -\frac{c_{I}}{b_{I}} & 0 & 0 & 0 \\
0 & 0 & 0 & 0 & 1 & 0 \\
0 & 0 & 0 & 0 & 0 & 1 \\
0 & 0 & 0 & 0 & -\frac{1}{b_{I I}} & -\frac{c_{I I}}{b_{I I}}
\end{array}\right]
$$

A matriz (5.92) é idêntica à matriz (5.9). Dessa forma, todos os resultados pertinentes ao estudo da estabilidade do ponto $P$ podem ser aqui aplicados da mesma forma. Para proceder ao estudo da estabilidade, será aplicado o TVC.

\subsubsection{ANÁLISE VIA TVC}

O sistema (5.91) pode ser representado matricialmente como $\dot{\bar{X}}=J_{P} \cdot \bar{X}+\bar{F}$, ou seja:

$$
\begin{aligned}
& {\left[\begin{array}{c}
\dot{\bar{x}}_{1} \\
\dot{\bar{x}}_{2} \\
\dot{\bar{x}}_{3} \\
\dot{\bar{x}}_{4} \\
\dot{\bar{x}}_{5} \\
\dot{\bar{x}}_{6}
\end{array}\right]=\left[\begin{array}{cccccc}
0 & 1 & 0 & 0 & 0 & 0 \\
0 & 0 & 1 & 0 & 0 & 0 \\
0 & -\frac{1}{b_{I}} & -\frac{c_{I}}{b_{I}} & 0 & 0 & 0 \\
0 & 0 & 0 & 0 & 1 & 0 \\
0 & 0 & 0 & 0 & 0 & 1 \\
0 & 0 & 0 & 0 & -\frac{1}{b_{I I}} & -\frac{c_{I I}}{b_{I I}}
\end{array}\right] \cdot\left[\begin{array}{c}
\bar{x}_{1} \\
\bar{x}_{2} \\
\bar{x}_{3} \\
\bar{x}_{4} \\
\bar{x}_{5} \\
\bar{x}_{6}
\end{array}\right]+} \\
& +\left[\begin{array}{c}
0 \\
0 \\
-\frac{k_{I} \cdot \Omega \cdot a_{I}}{b_{I}} \bar{x}_{2} \pm \frac{a_{I} \cdot \Omega}{b_{I}} \bar{x}_{1} \cdot \bar{x}_{2} \mp \frac{\Omega \cdot k_{I}}{b_{I}} \bar{x}_{1} \\
0 \\
0 \\
\left(\begin{array}{c}
\mp \frac{k_{I I} \cdot \Omega}{b_{I I}} \bar{x}_{1}+\frac{a_{I I} \cdot \Omega}{b_{I I}} \bar{x}_{1} \cdot \bar{x}_{2}- \\
-\frac{a_{I I} \cdot \Omega}{b_{I I}} \bar{x}_{1} \cdot \bar{x}_{5} \mp \frac{a_{I I} \cdot k_{I I} \cdot \Omega}{b_{I I}} \bar{x}_{2} \pm \frac{a_{I I} \cdot k_{I I} \cdot \Omega}{b_{I I}} \bar{x}_{5}
\end{array}\right)
\end{array}\right] \text { e } \dot{\Omega}=0
\end{aligned}
$$


Conforme requerido pelo TVC, o sistema (5.93) precisa ser reescrito na forma canônica de Jordan. Para isso, define-se um novo conjunto de variáveis de estado $V=\left(v_{1}, v_{2}, v_{3}, v_{4}, v_{5}, v_{6}\right)^{T}$ de modo que $\bar{X}=M \cdot V$, sendo $M$ uma base composta pelos autovetores de $J_{P}$ de (5.10). Assim, é possível se estabelecer a seguinte relação entre as variáveis de estado $X$ e $V$ :

$$
\begin{aligned}
& \bar{x}_{1}=v_{2}+\frac{1}{2}\left(c_{I} \cdot \delta_{I}-2 b_{I}\right) v_{3}+\frac{1}{2}\left(c_{I} \cdot \varepsilon_{I}-2 b_{I}\right) v_{4} \\
& \bar{x}_{2}=\frac{1}{2} \delta_{I} \cdot v_{3}-\frac{1}{2} \varepsilon_{I} \cdot v_{4} \\
& \bar{x}_{3}=v_{3}+v_{4} \\
& \bar{x}_{4}=v_{1}+\frac{1}{2}\left(c_{I I} \cdot \delta_{I I}-2 b_{I I}\right) v_{5}+\frac{1}{2}\left(c_{I I} \cdot \varepsilon_{I I}-2 b_{I I}\right) v_{6} \\
& \bar{x}_{5}=\frac{1}{2} \delta_{I I} \cdot v_{5}-\frac{1}{2} \varepsilon_{I I} \cdot v_{6} \\
& \bar{x}_{6}=v_{5}+v_{6}
\end{aligned}
$$

Sendo que em (5.94), tem-se $\delta=c-\sqrt{c^{2}-4 b}$ e $\varepsilon=c+\sqrt{c^{2}-4 b}$.

Dessa forma, é possível fazer $V=\left[M^{-1} \cdot J_{P} \cdot M\right] \cdot V+M^{-1} \cdot \bar{F}$ e $\dot{\Omega}=0$, ou seja:

$$
\left[\begin{array}{l}
\dot{v}_{1} \\
\dot{v}_{2} \\
\dot{v}_{3} \\
\dot{v}_{4} \\
\dot{v}_{5} \\
\dot{v}_{6}
\end{array}\right]=\left[\begin{array}{cccccc}
\lambda_{1} & 0 & 0 & 0 & 0 & 0 \\
0 & \lambda_{2} & 0 & 0 & 0 & 0 \\
0 & 0 & \lambda_{3} & 0 & 0 & 0 \\
0 & 0 & 0 & \lambda_{4} & 0 & 0 \\
0 & 0 & 0 & 0 & \lambda_{5} & 0 \\
0 & 0 & 0 & 0 & 0 & \lambda_{6}
\end{array}\right] \cdot\left[\begin{array}{l}
v_{1} \\
v_{2} \\
v_{3} \\
v_{4} \\
v_{5} \\
v_{6}
\end{array}\right]+\left[\begin{array}{l}
f_{1} \\
f_{2} \\
f_{3} \\
f_{4} \\
f_{5} \\
f_{6}
\end{array}\right] \text { e } \dot{\Omega}=0
$$

Em (5.95), tem-se que:

- $\lambda_{1}=0$

- $\lambda_{2}=0$ 
- $\lambda_{3}=-\frac{\left(c_{I}+\sqrt{c_{I}^{2}-4 b_{I}}\right)}{2 b_{I}}$

- $\lambda_{4}=\frac{\left(\sqrt{c_{I}^{2}-4 b_{I}}-c_{I}\right)}{2 b_{I}}$

- $\lambda_{5}=-\frac{\left(c_{I I}+\sqrt{c_{I I}^{2}-4 b_{I I}}\right)}{2 b_{I I}}$

- $\lambda_{6}=\frac{\left(\sqrt{c_{I I}^{2}-4 b_{I I}}-c_{I I}\right)}{2 b_{I I}}$

- $\left[\begin{array}{l}f_{1} \\ f_{2} \\ f_{3} \\ f_{4} \\ f_{5} \\ f_{6}\end{array}\right]=M^{-1} \cdot \bar{F}$

Por questão de simplicidade, optou-se por omitir os termos não-lineares $f_{1}$, $f_{2}, f_{3}, f_{4}, f_{5}$ e $f_{6}$ devido a sua extensão.

Então, de acordo com o Teorema da Variedade Central (Guckenheimer e Holmes, 1983; Wiggins, 1990; Monteiro, 2002), procura-se a variedade central $W^{C}$ que seja tangente subespaço central $E^{C}$ no ponto de equilíbrio $P$. Assim, para $y, z$ e $\Omega$ suficientemente pequenos, tem-se:

$$
\begin{aligned}
W^{c}(0)=\{ & \left(v_{1}, v_{2}, v_{3}, v_{4}, v_{5}, v_{6}\right) \in \mathfrak{R}^{4} / v_{1}=y, v_{2}=z, \\
& v_{3}=h_{1}(y, z, \Omega), v_{4}=h_{2}(y, z, \Omega), \\
& v_{5}=h_{3}(y, z, \Omega), v_{6}=h_{4}(y, z, \Omega), \\
& \left.h_{j}(0,0,0)=0, D h_{j}(0,0,0)=0, j=1,2,3,4\right\}
\end{aligned}
$$

Considere-se então, os seguintes polinômios estimadores compostos por monômios do segundo grau $h_{1}(y, z, \Omega), h_{2}(y, z, \Omega), h_{3}(y, z, \Omega)$ e $h_{4}(y, z, \Omega)$ :

$$
\begin{aligned}
& h_{1}(y, z, \Omega)=\alpha_{1} \cdot y^{2}+\alpha_{2} \cdot z^{2}+\alpha_{3} \cdot \Omega^{2}+\alpha_{4} \cdot y \cdot z+\alpha_{5} \cdot y \cdot \Omega \\
& \quad+\alpha_{6} \cdot z \cdot \Omega,\left(\alpha_{j} \in \mathbb{R}, j=1, \ldots, 7\right)
\end{aligned}
$$




$$
\begin{aligned}
& h_{2}(y, z, \Omega)=\beta_{1} \cdot y^{2}+\beta_{2} \cdot z^{2}+\beta_{3} \cdot \Omega^{2}+\beta_{4} \cdot y \cdot z+\beta_{5} \cdot y \cdot \Omega \\
& \quad+\beta_{6} \cdot z \cdot \Omega,\left(\beta_{j} \in \mathbb{R}, j=1, \ldots, 7\right) \\
& h_{3}(y, z, \Omega)=\gamma_{1} \cdot y^{2}+\gamma_{2} \cdot z^{2}+\gamma_{3} \cdot \Omega^{2}+\gamma_{4} \cdot y \cdot z+\gamma_{5} \cdot y \cdot \Omega \\
& \quad+\gamma_{6} \cdot z \cdot \Omega,\left(\gamma_{j} \in \mathbb{R}, j=1, \ldots, 7\right) \\
& h_{3}(y, z, \Omega)=\theta_{1} \cdot y^{2}+\theta_{2} \cdot z^{2}+\theta_{3} \cdot \Omega^{2}+\theta_{4} \cdot y \cdot z+\theta_{5} \cdot y \cdot \Omega \\
& \quad+\theta_{6} \cdot z \cdot \Omega,\left(\theta_{j} \in \mathbb{R}, j=1, \ldots, 7\right)
\end{aligned}
$$

Pelo teorema de Carr (Carr, 1981; Guckenheimer e Holmes, 1983; Wiggins, 1990), a variedade central $W^{c}$ deve satisfazer $N(h(y, z, \Omega))=0$. Portanto, tem-se:

$$
\begin{aligned}
& \frac{\partial h_{1}(y, z, \Omega)}{\partial y}\left[\lambda_{1} \cdot x+f_{1}\left(y, z, \Omega, h_{1}, h_{2}, h_{3}, h_{4}\right)\right]+ \\
& +\frac{\partial h_{1}(y, z, \Omega)}{\partial z}\left[\lambda_{2} \cdot x+f_{2}\left(y, z, \Omega, h_{1}, h_{2}, h_{3}, h_{4}\right)\right]- \\
& -\lambda_{3} \cdot h_{1}(y, z, \Omega)-f_{3}\left(y, z, \Omega, h_{1}, h_{2}, h_{3}, h_{4}\right)=0 \\
& \frac{\partial h_{2}(y, z, \Omega)}{\partial z}\left[\lambda_{2} \cdot x+f_{2}\left(y, z, \Omega, h_{1}, h_{2}, h_{3}, h_{4}\right)\right]+ \\
& +\frac{\partial h_{2}(y, z, \Omega)}{\partial z}\left[\lambda_{2} \cdot x+f_{2}\left(y, z, \Omega, h_{1}, h_{2}, h_{3}, h_{4}\right)\right]- \\
& -\lambda_{4} \cdot h_{2}(y, z, \Omega)-f_{4}\left(y, z, \Omega, h_{1}, h_{2}, h_{3}, h_{4}\right)=0 \\
& \frac{\partial h_{3}(y, z, \Omega)}{\partial y}\left[\lambda_{1} \cdot x+f_{1}\left(y, z, \Omega, h_{1}, h_{2}, h_{3}, h_{4}\right)\right]+ \\
& +\frac{\partial h_{3}(y, z, \Omega)}{\partial z}\left[\lambda_{2} \cdot x+f_{2}\left(y, z, \Omega, h_{1}, h_{2}, h_{3}, h_{4}\right)\right]- \\
& -\lambda_{4} \cdot h_{5}(y, z, \Omega)-f_{4}\left(y, z, \Omega, h_{1}, h_{2}, h_{3}, h_{4}\right)=0 \\
& \frac{\partial h_{4}(y, z, \Omega)}{\partial z}\left[\lambda_{2} \cdot x+f_{2}\left(y, z, \Omega, h_{1}, h_{2}, h_{3}, h_{4}\right)\right] \\
& +\frac{\partial h_{4}(y, z, \Omega)}{\partial z}\left[\lambda_{2} \cdot x+f_{2}\left(y, z, \Omega, h_{1}, h_{2}, h_{3}, h_{4}\right)\right]- \\
& -\lambda_{6} \cdot h_{4}(y, z, \Omega)-f_{6}\left(y, z, \Omega, h_{1}, h_{2}, h_{3}, h_{4}\right)=0
\end{aligned}
$$

Utilizando (5.95) e (5.97) em (5.98) e resolvendo o sistema de equações, é possível obter, igualando-se a zero os termos de igual potência, os coeficientes de $h_{1}(y, z, \Omega), h_{2}(y, z, \Omega), h_{3}(y, z, \Omega)$ e $h_{4}(y, z, \Omega)$ : 


$$
\begin{aligned}
& y^{2}: \alpha_{1}=0, \beta_{1}=0, \gamma_{1}=0, \theta_{1}=0 \\
& z^{3}: \alpha_{2}=0, \beta_{2}=0, \gamma_{2}=0, \theta_{2}=0 \\
& \Omega^{2}: \alpha_{3}=0, \beta_{3}=0, \gamma_{3}=0, \theta_{3}=0 \\
& y \cdot z: \alpha_{4}=0, \beta_{4}=0, \gamma_{4}=0, \theta_{4}=0 \\
& y \cdot \Omega: \alpha_{5}=\mp \frac{\sqrt{c_{I}^{2}-4 b_{I}}}{c_{I}^{2}-4 b_{I}} k_{I}, \beta_{5}= \pm \frac{\sqrt{c_{I}^{2}-4 b_{I}}}{c_{I}^{2}-4 b_{I}} k_{I}, \\
& \gamma_{5}=\mp \frac{\sqrt{c_{I I}^{2}-4 b_{I I}}}{c_{I I}^{2}-4 b_{I I}} k_{I I}, \theta_{5}= \pm \frac{\sqrt{c_{I I}^{2}-4 b_{I I}}}{c_{I I}^{2}-4 b_{I I}} k_{I I} \\
& z \cdot \Omega: \alpha_{6}=0, \beta_{6}=0, \gamma_{6}=0, \theta_{6}=0
\end{aligned}
$$

Com os coeficientes (5.99) dos polinômios de (5.97), pode-se obter, através do sistema (5.95), o campo de vetores restrito à variedade central, desprezando-se os termos de ordem superior:

$$
\left\{\begin{aligned}
\dot{y}= & z \cdot \Omega\left[-a_{I I} \cdot \Omega \cdot k_{I I}^{2}+\left(-c_{I} \cdot k_{I} \cdot \Omega+a_{I I}\left(c_{I} \cdot z \cdot \Omega \cdot k_{I}+k_{I} \pm z\right) \Omega \mp 1\right) k_{I I} \mp\right. \\
& \left.\mp a_{I I} \cdot k_{I} \cdot z \cdot \Omega\left( \pm c_{I} \cdot k_{I} \cdot \Omega+1\right)\right] \\
\dot{z}= & -k_{I} \cdot z \cdot \Omega\left[c_{I} \cdot k_{I} \cdot \Omega+a_{I}\left( \pm z+k_{I}\left(c_{I} \cdot z \cdot \Omega \mp 1\right)\right) \Omega \pm 1\right]
\end{aligned}\right.
$$

Para obter os pontos de equilíbrio de (5.100), basta fazer:

$$
\left\{\begin{array}{l}
\dot{y}=0 \\
\dot{z}=0
\end{array}\right.
$$

De (5.101) se obtém:

$$
y=r, \forall r \in \mathbb{R} \text { e } z=0
$$

Para o estudo da estabilidade do ponto de equilíbrio de (5.102), é preciso obter a matriz Jacobiana de (5.100):

$$
J_{P}=\left[\begin{array}{ll}
\left.\frac{\partial \dot{y}}{\partial y}\right|_{P} & \left.\frac{\partial \dot{y}}{\partial z}\right|_{P} \\
\left.\frac{\partial \dot{z}}{\partial y}\right|_{P} & \left.\frac{\partial \dot{z}}{\partial z}\right|_{P}
\end{array}\right]
$$


Sendo que em (5.103), tem-se:

$$
\begin{aligned}
\frac{\partial \dot{y}}{\partial y}= & \\
\frac{\partial \dot{y}}{\partial z}= & \Omega\left[-a_{I I} \cdot \Omega \cdot k_{I I}^{2}+\left(-c_{I} \cdot k_{I} \cdot \Omega+a_{I I}\left(2 c_{I} \cdot z \cdot \Omega \cdot k_{I}+k_{I}+2 z\right) \Omega-1\right) k_{I I}-\right. \\
& \left.-2 a_{I I} \cdot k_{I} \cdot z \cdot \Omega\left(c_{I} \cdot k_{I} \cdot \Omega+1\right)\right] \\
\frac{\partial \dot{z}}{\partial y}= & 0 \\
\frac{\partial \dot{z}}{\partial z}= & -k_{I} \cdot \Omega\left[c_{I} \cdot k_{I} \cdot \Omega+a_{I}\left(2 z+k_{I}\left(2 c_{I} \cdot z \cdot \Omega-1\right)\right) \Omega+1\right]
\end{aligned}
$$

Em seguida, verifica-se a parte real dos autovalores para a matriz Jacobiana (5.103) calculada no ponto de equilíbrio:

$$
\begin{aligned}
& \lambda_{1}=0, \lambda_{2}=-k_{I} \cdot \Omega\left[c_{I} \cdot k_{I} \cdot \Omega+a_{I}\left(\mp 2 z+k_{I}\left(2 c_{I} \cdot z \cdot \Omega \mp 1\right)\right) \Omega \pm 1\right] \\
& \left(\operatorname{Re}\left(\lambda_{2}\right)>0\right) \Leftrightarrow \operatorname{Para} \mathrm{P} 7 \\
& \left(c_{I}<a_{I} \wedge\left(\left(k_{I}<0 \wedge\left(\Omega<\frac{1}{a_{I} \cdot k_{I}-c_{I} \cdot k_{I}} \vee \Omega>0\right)\right) \vee\right.\right. \\
& \left.\left.\vee\left(k_{I}>0 \wedge\left(\Omega>\frac{1}{a_{I} \cdot k_{I}-c_{I} \cdot k_{I}} \vee \Omega<0\right)\right)\right)\right) \vee \\
& \vee\left(c_{I}=a_{I} \wedge\left(\left(k_{I}<0 \wedge \Omega>0\right) \vee\left(k_{I}>0 \wedge \Omega<0\right)\right)\right) \vee \\
& \vee\left(c_{I}>a_{I} \wedge\left(\left(k_{I}<0 \wedge 0<\Omega<\frac{1}{a_{I} \cdot k_{I}-c_{I} \cdot k_{I}}\right) \vee\right.\right. \\
& \left.\left.\vee\left(k_{I}>0 \wedge \frac{1}{a_{I} \cdot k_{I}-c_{I} \cdot k_{I}}<\Omega<0\right)\right)\right)
\end{aligned}
$$

Para P8,

$$
\left(c_{I}<-a_{I} \wedge\left(\left(k_{I}<0 \wedge\left(\Omega>\frac{1}{a_{I} \cdot k_{I}+c_{I} \cdot k_{I}} \vee \Omega<0\right)\right) \vee\right.\right.
$$




$$
\begin{aligned}
& \left.\left.\vee\left(k_{I}>0 \wedge\left(\Omega<\frac{1}{a_{I} \cdot k_{I}+c_{I} \cdot k_{I}} \vee \Omega>0\right)\right)\right)\right) \vee \\
& \vee\left(c_{I}=-a_{I} \wedge\left(\left(k_{I}<0 \wedge \Omega<0\right) \vee\left(k_{I}>0 \wedge \Omega>0\right)\right)\right) \vee \\
& \vee\left(c_{I}>-a_{I} \wedge\left(\left(k_{I}<0 \wedge \frac{1}{a_{I} \cdot k_{I}+c_{I} \cdot k_{I}}<\Omega<0\right) \vee\right.\right. \\
& \left.\left.\vee\left(k_{I}>0 \wedge 0<\Omega<\frac{1}{a_{I} \cdot k_{I}+c_{I} \cdot k_{I}}\right)\right)\right)
\end{aligned}
$$

A interpretação analítica e gráfica dos resultados apresentados será realizada no capítulo VI. 


\section{CAPÍTULO VI}

\section{SÍNTESE DOS RESULTADOS}

Neste capítulo será apresentada uma síntese dos resultados obtidos nos capítulos II, IV e V. Entretanto, as relações mais gerais que foram obtidas nesses capítulos agora serão refeitas, considerando-se valores não-hipotéticos para os parâmetros. Estes resultados, mais apurados, serão ilustrados através dos respectivos diagramas de bifurcações.

\subsection{SINCRONISMO PARA UMA VARIAÇÃO DO TIPO DEGRAU DE FASE NO SINAL DO MESTRE}

\subsubsection{SINCRONISMO DE UM NÓ TIPO I}

No estudo do sincronismo de um nó tipo I, isto é, diretamente conectado ao mestre, dois pontos de equilíbrio foram obtidos, $P_{1}=(0,0,0)$ e $P_{2}=(-\pi, 0,0)$.

$\mathrm{O}$ campo de vetores restrito à variedade central em torno de $\mathrm{P}_{1}$ é:

$$
\dot{x}=-\mu_{I} \cdot x
$$

Assim, o ponto de equilíbrio $\mathrm{P}_{1}$ é assintoticamente estável para $\mu_{I}>0 \mathrm{e}$ instável, para $\mu_{I}<0$. O diagrama de bifurcações correspondente à (6.1) encontra-se a seguir.

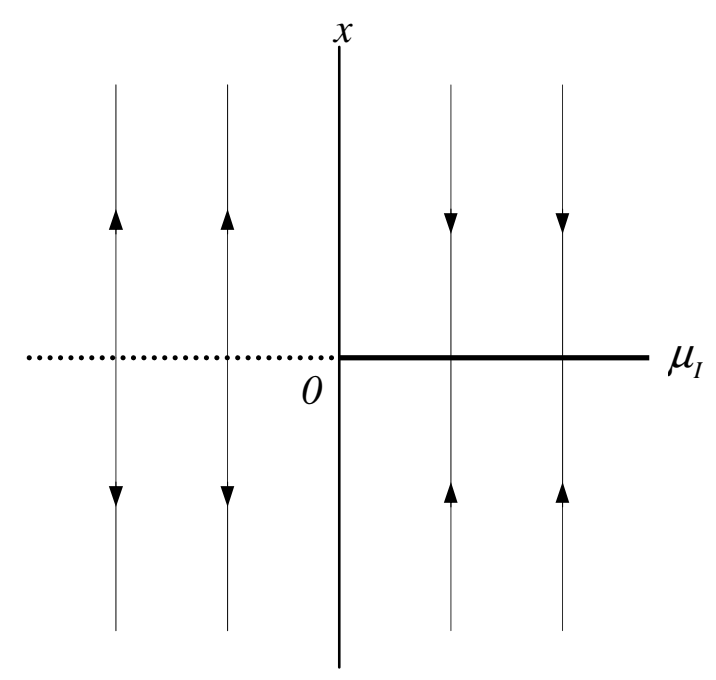

Fig.6.1: Diagrama de bifurcações para $P_{1}$. As setas indicam a evolução temporal de $x(t)$, para um dado valor de $\mu_{I}$ e para uma dada condição inicial sobre a seta. 
$\mathrm{O}$ campo de vetores restrito à variedade central em torno de $\mathrm{P}_{2}$ é:

$$
\dot{x}=+\mu_{I} \cdot x
$$

Assim, o ponto de equilíbrio $\mathrm{P}_{2}$ é instável para $\mu_{I}>0$ e assintoticamente estável para $\mu_{I}<0$. O diagrama de bifurcações correspondente a (6.2) encontra-se a seguir.

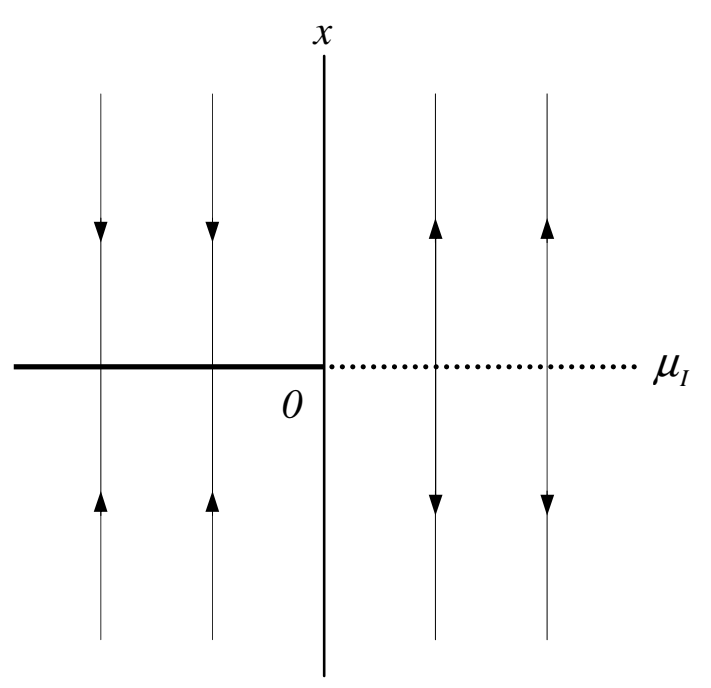

Fig.6.2: Diagrama de bifurcações para $P_{2}$. As setas indicam a evolução temporal de $x(t)$, para um dado valor de $\mu_{I}$ e para uma dada condição inicial sobre a seta.

Como é possível observar, a estabilidade do sincronismo se deve, em princípio, exclusivamente ao parâmetro $\mu_{I}$, dado que seja suficientemente pequeno.

Por outro lado, o parâmetro $\mu_{I}$ foi definido como sendo $\mu_{I} \stackrel{\Delta}{=} \frac{K_{I} \cdot K_{m, I} \cdot V_{M} \cdot V_{I}}{2}$, ou seja, é o produto entre os seguintes fatores positivos nãonulos:

- $\quad K_{m, I}$, ganho do PD do nó I;

- $K_{I}$, ganho do VCO do nó I;

- $\quad V_{M}$, amplitude do sinal do mestre

- $\quad V_{I}$, amplitude do sinal do nó I, assume-se que

Portanto, na prática, o parâmetro $\mu_{I}$ também é considerado positivo nãonulo. A conclusão para este caso é bastante simples, portanto. Para uma variação do 
tipo degrau de fase no nó mestre, o sistema dinâmico que descreve o sincronismo de um nó tipo I possui dois pontos de equilíbrio, sendo que um deles, $\mathrm{P}_{1}$, é assintoticamente estável e o outro, $\mathrm{P}_{2}$, é instável para qualquer valor que possa assumir o parâmetro $\mu_{I}$. Também é importante observar que o parâmetro $b_{I}$ do filtro, cuja função de transferência é $F_{I}(s)=\frac{a_{I} \cdot s+1}{b_{I} \cdot s^{2}+c_{I} \cdot s+1}$, deve ser não-nulo, condição que foi, inclusive, assumida na obtenção do sistema de equações no espaço de fases. Ainda, a estabilidade deste PLL não depende diretamente deste parâmetro. Os retratos de fases correspondentes à (6.1) e à (6.2) ilustram, para $\mu_{I}>0$, a estabilidade de $\mathrm{P}_{1}$ e de $\mathrm{P}_{2}$ :

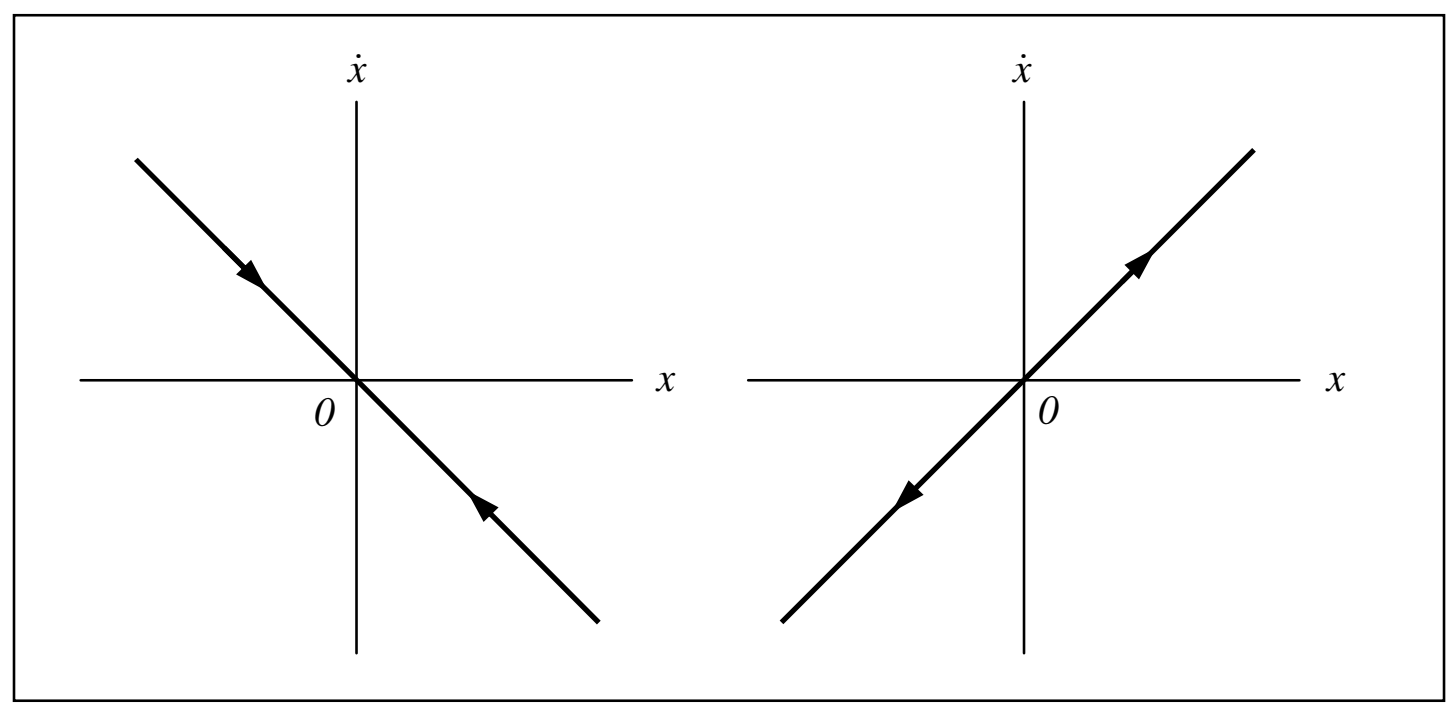

Fig.6.3: Retrato de fases em $P_{1}$ e $P_{2}$, para $\mu_{I}>0$.

Neste retrato, o sentido das setas indica a evolução temporal do sistema a partir de todas as condições iniciais definidas para (2.60) e (2.64), respectivamente.

\subsubsection{SINCRONISMO DE UM NÓ TIPO II}

No estudo do sincronismo de um nó tipo II, isto é, diretamente conectado a um nó tipo I, obteve-se quatro pontos de equilíbrio:

- $P_{1}=(0,0,0,0,0,0)$

- $\quad P_{2}=(-\pi, 0,0,0,0,0)$

- $\quad P_{3}=(-\pi, 0,0,-\pi, 0,0)$ 
- $\quad P_{2}=(0,0,0,-\pi, 0,0)$

$\mathrm{O}$ campo de vetores restrito à variedade central em torno de $\mathrm{P}_{1}$ é:

$\left\{\begin{array}{l}\dot{y}=-\mu_{I I} \cdot y+\mu_{I I} \cdot z \\ \dot{z}=-\mu_{I} \cdot z\end{array}\right.$

Assim, para $\mu_{I}>0$ e $\mu_{I I}>0$ o ponto de equilíbrio $\mathrm{P}_{1}$ é assintoticamente estável e, instável, nos outros casos. Os diagramas de bifurcações correspondentes à (6.3) encontram-se a seguir.
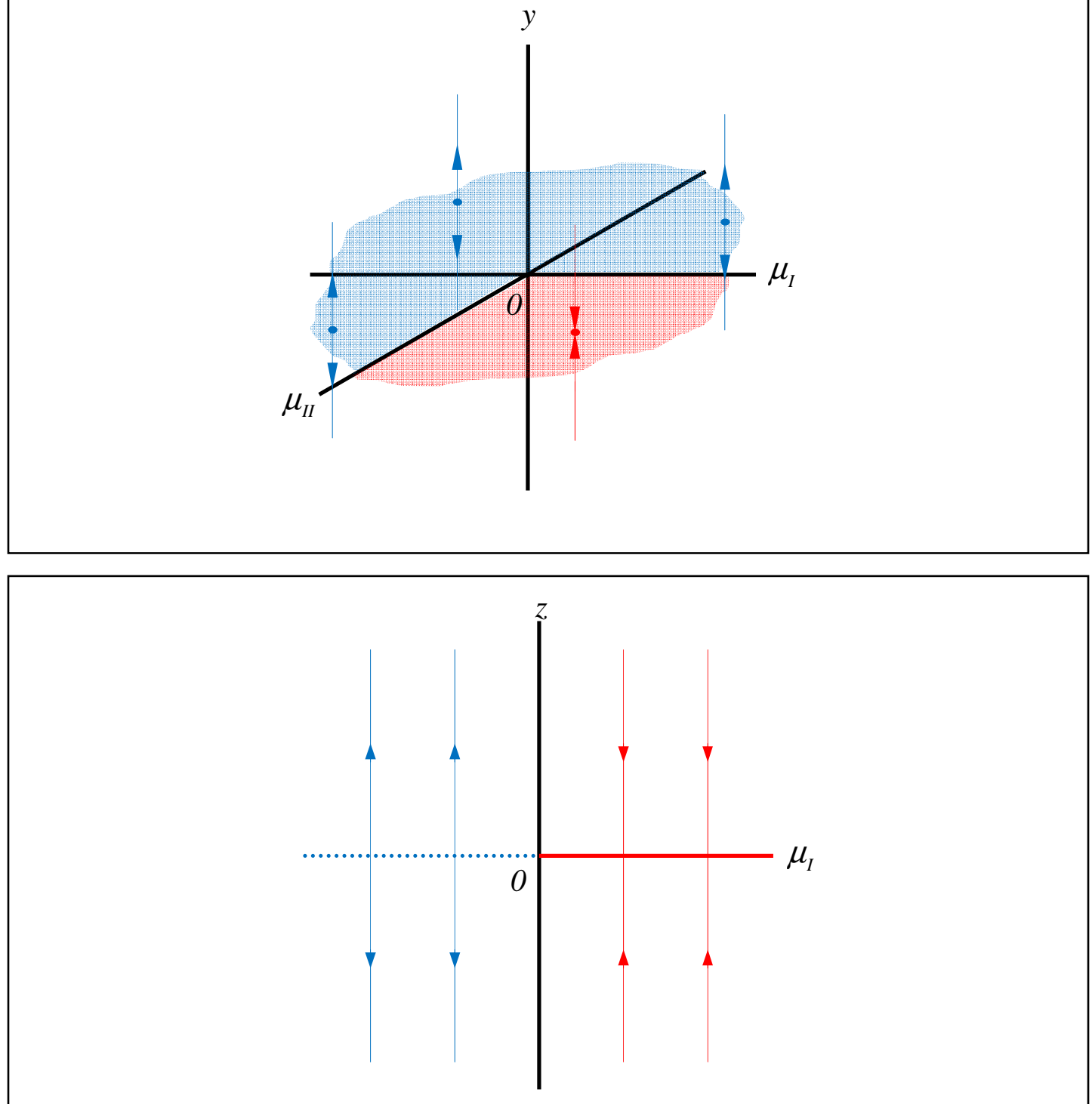

Fig.6.4: Diagramas de bifurcações para $P_{1}$. As setas indicam a evolução temporal de $y(t) ; z(t)$, para um dado valor de $\mu_{I}$ e $\mu_{I I}$ e para uma dada condição inicial sobre a seta. 
$\mathrm{O}$ campo de vetores restrito à variedade central em torno de $\mathrm{P}_{2}$ é:

$\left\{\begin{array}{l}\dot{y}=+\mu_{I I} \cdot y-\mu_{I I} \cdot z \\ \dot{z}=+\mu_{I} \cdot z\end{array}\right.$

Assim, $\mu_{I}<0$ e $\mu_{I I}<0$ o ponto de equilíbrio $\mathrm{P}_{2}$ é assintoticamente estável e, instável, nos outros casos. Os diagramas de bifurcações correspondentes à (6.4) encontram-se a seguir.
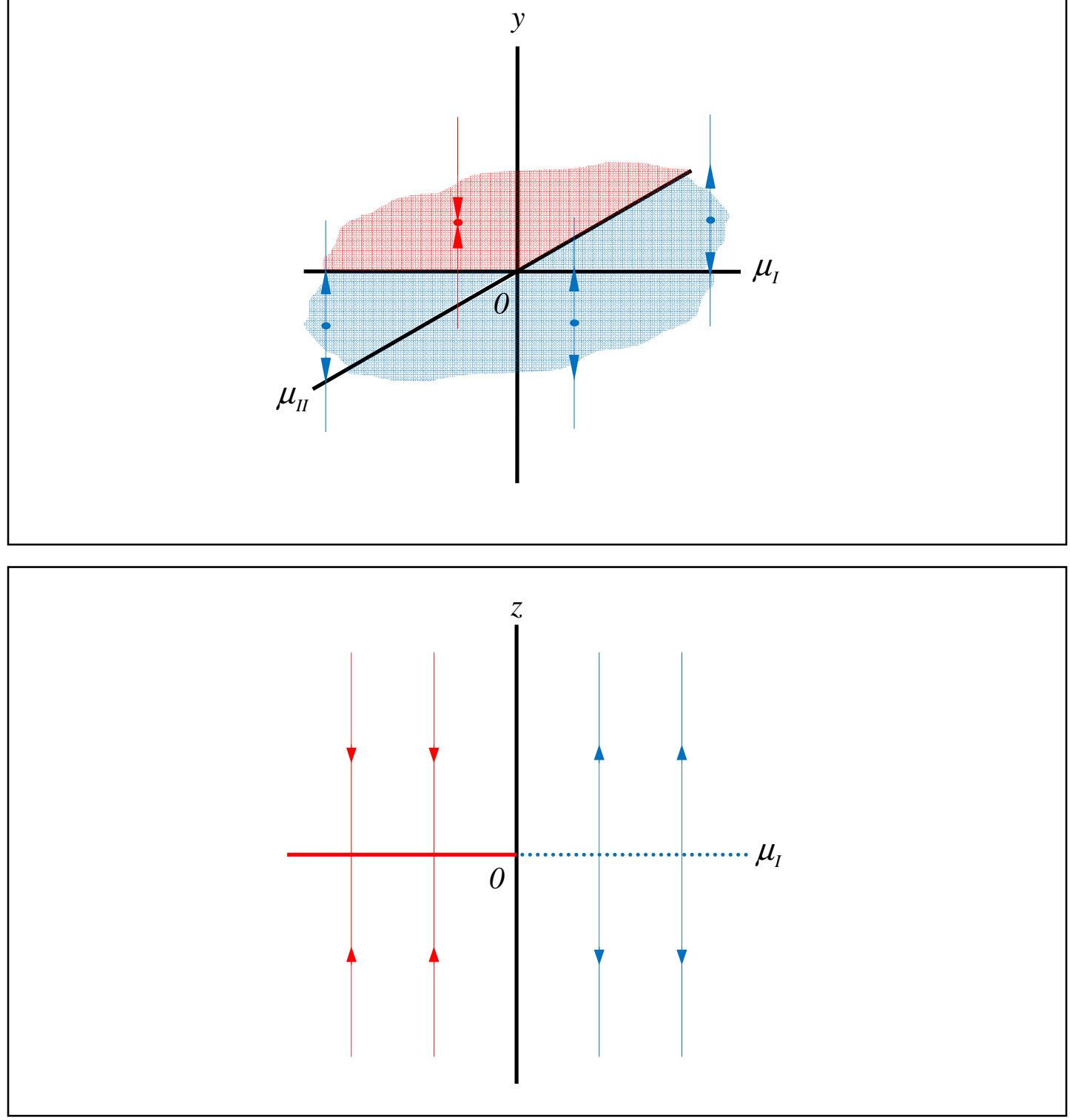

Fig.6.6: Diagramas de bifurcações para $P_{2}$. As setas indicam a evolução temporal de $y(t) ; z(t)$, para um dado valor de $\mu_{I}$ e $\mu_{I I}$ e para uma dada condição inicial sobre a seta.

$\mathrm{O}$ campo de vetores restrito à variedade central em torno de $\mathrm{P}_{3}$ é: 


$$
\left\{\begin{array}{l}
\dot{y}=-\mu_{I I} \cdot y+\mu_{I I} \cdot z \\
\dot{z}=+\mu_{I} \cdot z
\end{array}\right.
$$

Assim, $\mu_{I}<0$ e $\mu_{I I}>0$ o ponto de equilíbrio $\mathrm{P}_{3}$ é assintoticamente estável e, instável, nos outros casos. Os diagramas de bifurcações correspondentes à (6.5) encontram-se a seguir.
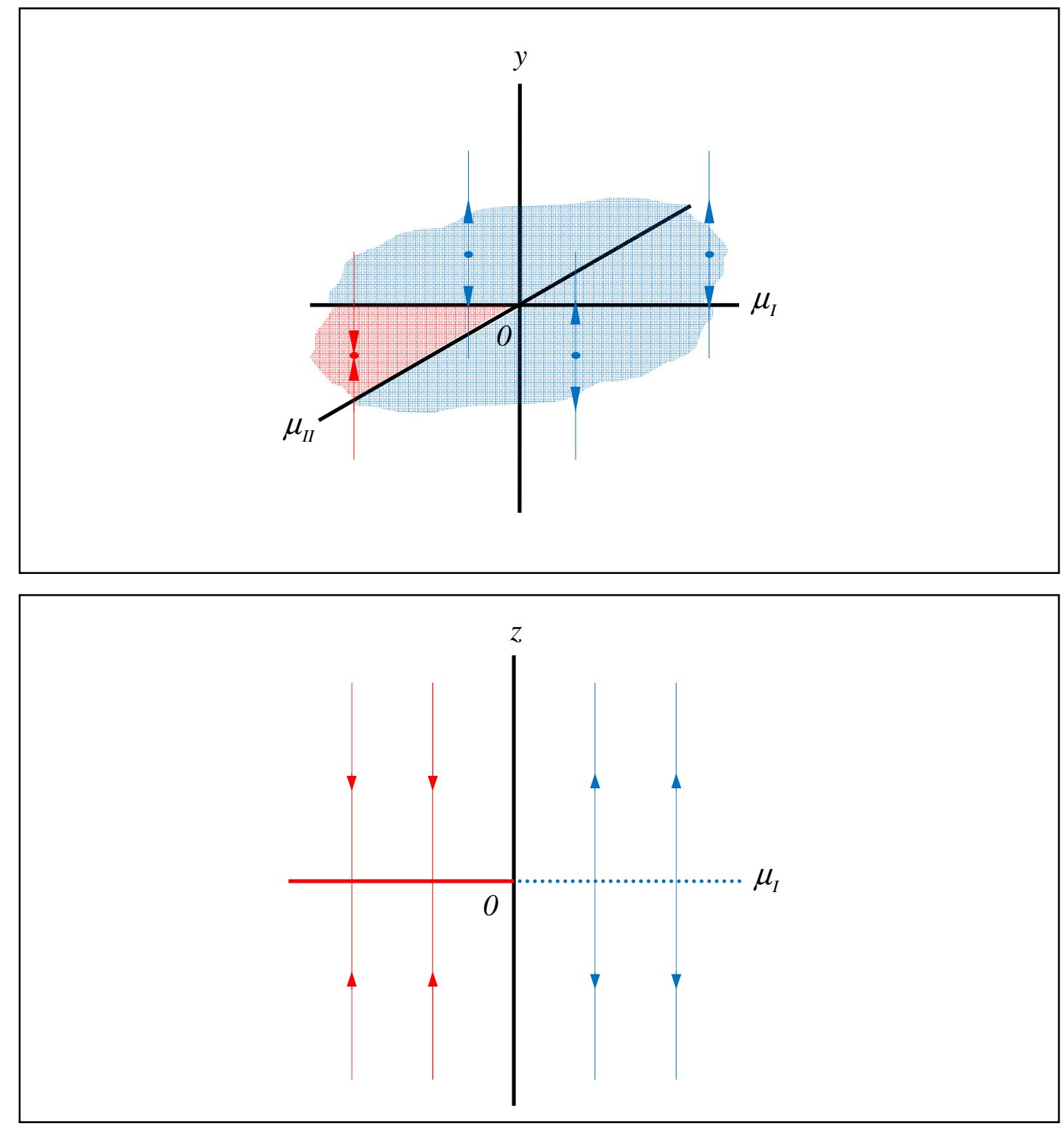

Fig.6.7: Diagramas de bifurcações para $P_{3}$. As setas indicam a evolução temporal de $y(t) ; z(t)$, para um dado valor de $\mu_{I}$ e $\mu_{I I}$ e para uma dada condição inicial sobre a seta.

O campo de vetores restrito à variedade central em torno de $\mathrm{P}_{4}$ é: 


$$
\left\{\begin{array}{l}
\dot{y}=+\mu_{I I} \cdot y-\mu_{I I} \cdot z \\
\dot{z}=-\mu_{I} \cdot z
\end{array}\right.
$$

Assim, $\mu_{I}<0$ e $\mu_{I I}>0$ o ponto de equilíbrio $\mathrm{P}_{4}$ é assintoticamente estável e, instável, nos outros casos. Os diagramas de bifurcações correspondentes à (6.5) encontram-se a seguir.
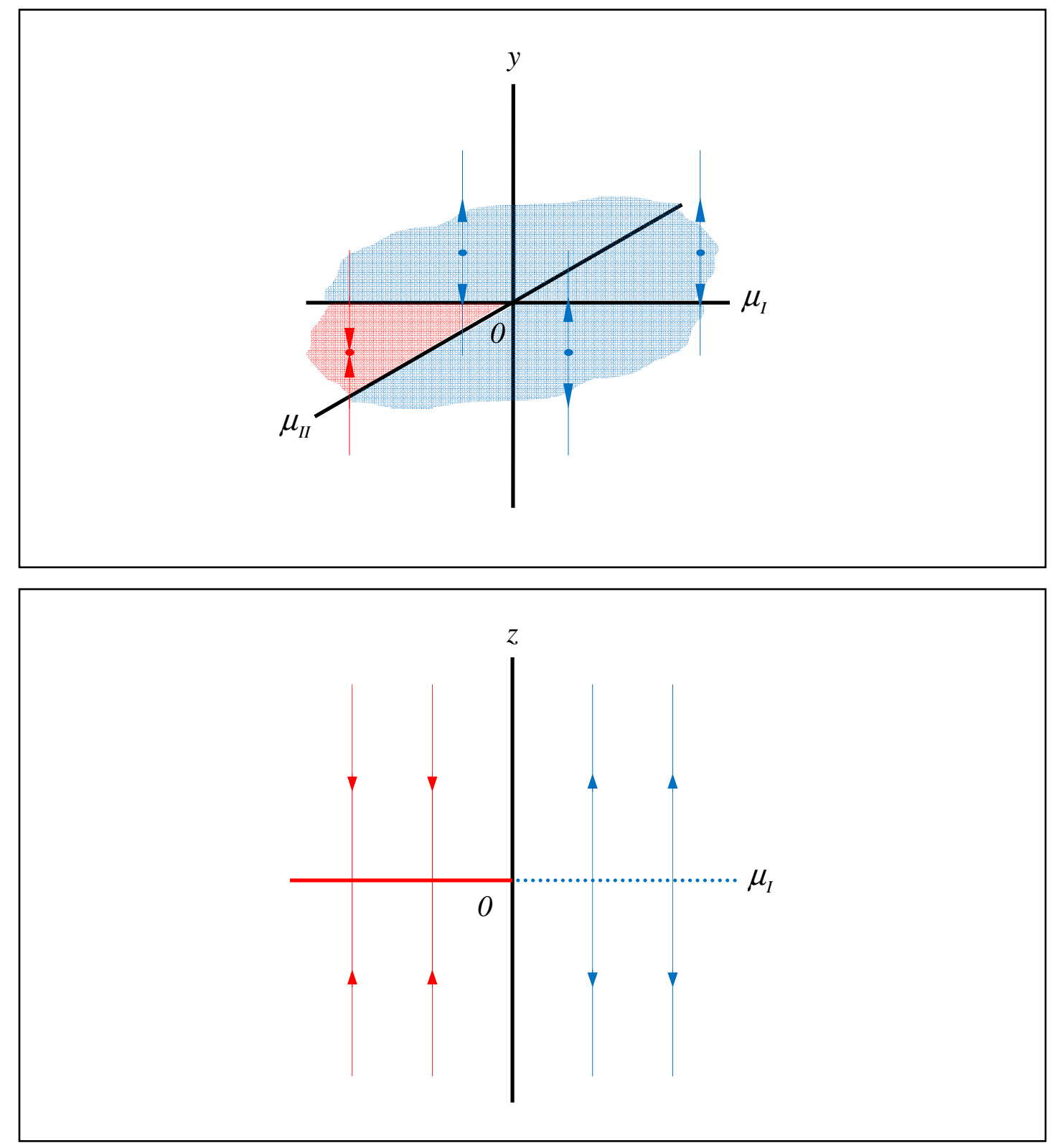

Fig.6.8: Diagramas de bifurcações para $P_{4}$. As setas indicam a evolução temporal de $y(t) ; z(t)$, para um dado valor de $\mu_{I}$ e $\mu_{I I}$ e para uma dada condição inicial sobre a seta.

Novamente, a estabilidade do sincronismo se deve, em princípio, exclusivamente aos parâmetros de $\mu_{I}$ e $\mu_{I I}$, dado que sejam suficientemente 
pequenos. É claro que o parâmetro $\mu_{I I}$ também é considerado positivo não-nulo. A conclusão para este caso também é bastante simples, portanto. Para uma variação do tipo degrau de fase no nó mestre, o sistema dinâmico que descreve o sincronismo de um nó tipo II possui quatro pontos de equilíbrio, sendo que um deles, $\mathrm{P}_{1}$, é assintoticamente estável e, os outros três, $\mathrm{P}_{2}, \mathrm{P}_{3}$ e $\mathrm{P}_{4}$, são instáveis para qualquer valor que possam assumir os parâmetros $\mu_{I}$ e $\mu_{I I}$. Mais uma vez, é importante ressaltar que os parâmetros $b_{I}$ e $b_{I I}$ dos filtros, respectivamente dos nós I e II, devem ser não-nulos, condição que foi assumida na obtenção do sistema de equações no espaço de fases. Ainda, a estabilidade destes PLLs não depende diretamente destes parâmetros.

\subsection{SINCRONISMO PARA UMA VARIAÇÃO DO TIPO RAMPA DE FASE NO SINAL DO MESTRE}

\subsubsection{SINCRONISMO DE UM NÓ TIPO I}

No estudo do sincronismo de um nó tipo I para uma rampa de fase no nó mestre de declividade $\Omega, \Omega>0$, obteve-se três pontos de equilíbrio:

- Se $\Omega=\mu_{I}$, então $P_{1}=\left(+\frac{\pi}{2}, 0,0\right)$

- Se $\Omega<\mu_{I}$, então $P_{2,3}=\left(\arccos \left( \pm \sqrt{1-\left(\frac{\Omega}{\mu_{I}}\right)^{2}}\right), 0,0\right)$

$\mathrm{O}$ campo de vetores restrito à variedade central, em torno de $\mathrm{P}_{1}$, é:

$$
\dot{x}=\frac{\mu_{I}^{2}\left(a_{I}-c_{I}\right)}{2} x^{3}+\frac{\mu_{I}}{2} x^{2}
$$

Assim, o ponto de equilíbrio $\mathrm{P}_{1}$, representado por $x^{*}=-\frac{1}{\left(a_{I}-c_{I}\right) \mu_{I}}$, é assintoticamente estável para $a_{I}>c_{I}$ e instável para $a_{I}<c_{I}$. O diagrama de bifurcação correspondente à (6.7) encontra-se a seguir. 


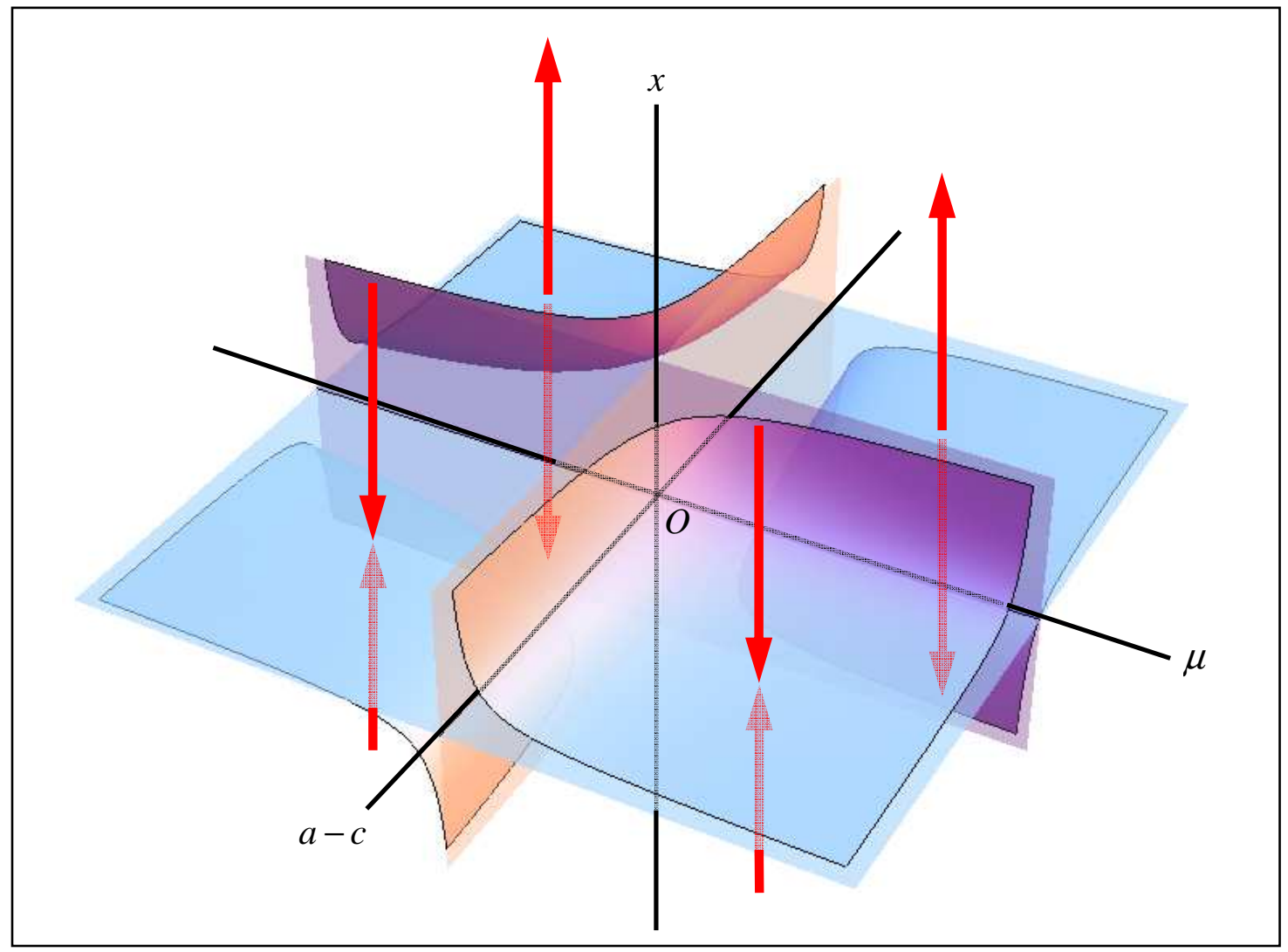

Fig.6.9: Diagrama de bifurcações para $P_{1}$. As setas indicam a evolução temporal de $x(t)$ para um dado valor de $(\mu,(a-c))$ e para uma dada condição inicial sobre a seta.

$\mathrm{O}$ campo de vetores restrito à variedade central, em torno de $\mathrm{P}_{2,3}$, é:

$$
\dot{x}=\frac{1}{2} x \cdot \Omega\left(x+k_{I}\left(c_{I}\left(c_{I}-2 a_{I}\right) k_{I} \cdot x \cdot \Omega^{2}+2\left(a_{I}-c_{I}\right)\left(k_{I} \mp x\right) \Omega \mp 2\right)\right)
$$

Sendo que em (6.8) tem-se que $k_{I} \stackrel{\Delta}{=} \sqrt{\frac{\mu_{I}^{2}}{\Omega^{2}}-1}$.

De (6.8) se obtém outros dois pontos de equilíbrio, $\mathrm{P}_{2 \mathrm{a}}$ e $\mathrm{P}_{3 \mathrm{a}}$, próximos, respectivamente, aos pontos $\mathrm{P}_{2}$ e $\mathrm{P}_{3}$ :

- $x_{1}^{*}=0$ (representando $\mathrm{P}_{2}$ e $\mathrm{P}_{3}$ )

- $x_{2}^{*}=\frac{k_{I}}{\left(c_{I}-2 a_{I}\right) k_{I} \cdot \Omega \pm 1}+\frac{k_{I}}{c_{I} \cdot k_{I} \cdot \Omega \pm 1}\left(\right.$ representando $\mathrm{P}_{2 \mathrm{a}}$ e $\left.\mathrm{P}_{3 \mathrm{a}}\right)$

De (6.9), observando que $k_{I}>0$, conclui-se que: 
- $\operatorname{Se}\left(\sqrt{\Omega^{2}+\frac{1}{\left(a_{I}-c_{I}\right)^{2}}}>\mu_{I} \vee a_{I} \leq c_{I}\right), \quad$ o ponto de equilíbrio $\mathrm{P}_{2}$ é assintoticamente estável e $\mathrm{P}_{2 \mathrm{a}}$, instável;

- $\operatorname{Se}\left(c_{I}<a_{I} \wedge \mu_{I}>\sqrt{\Omega^{2}+\frac{1}{\left(a_{I}-c_{I}\right)^{2}}}\right)$, o ponto de equilíbrio $\mathrm{P}_{2}$ é instável e $\mathrm{P}_{2 \mathrm{a}}$, assintoticamente estável;

- $\operatorname{Se}\left(c_{I}>a_{I} \wedge \mu_{I}>\sqrt{\Omega^{2}+\frac{1}{\left(a_{I}-c_{I}\right)^{2}}}\right), \quad$ o ponto de equilíbrio $\mathrm{P}_{3}$ assintoticamente estável e $\mathrm{P}_{3 a}$, instável;

- $\operatorname{Se}\left(\sqrt{\Omega^{2}+\frac{1}{\left(a_{I}-c_{I}\right)^{2}}}>\mu_{I} \wedge a_{I} \geq c_{I}\right)$, o ponto de equilíbrio $\mathrm{P}_{3}$ é instável e $\mathrm{P}_{3 \mathrm{a}}$, assintoticamente estável.

O diagrama de bifurcações correspondente à (6.10), (6.11), (6.12) e (6.13), encontra-se a seguir:

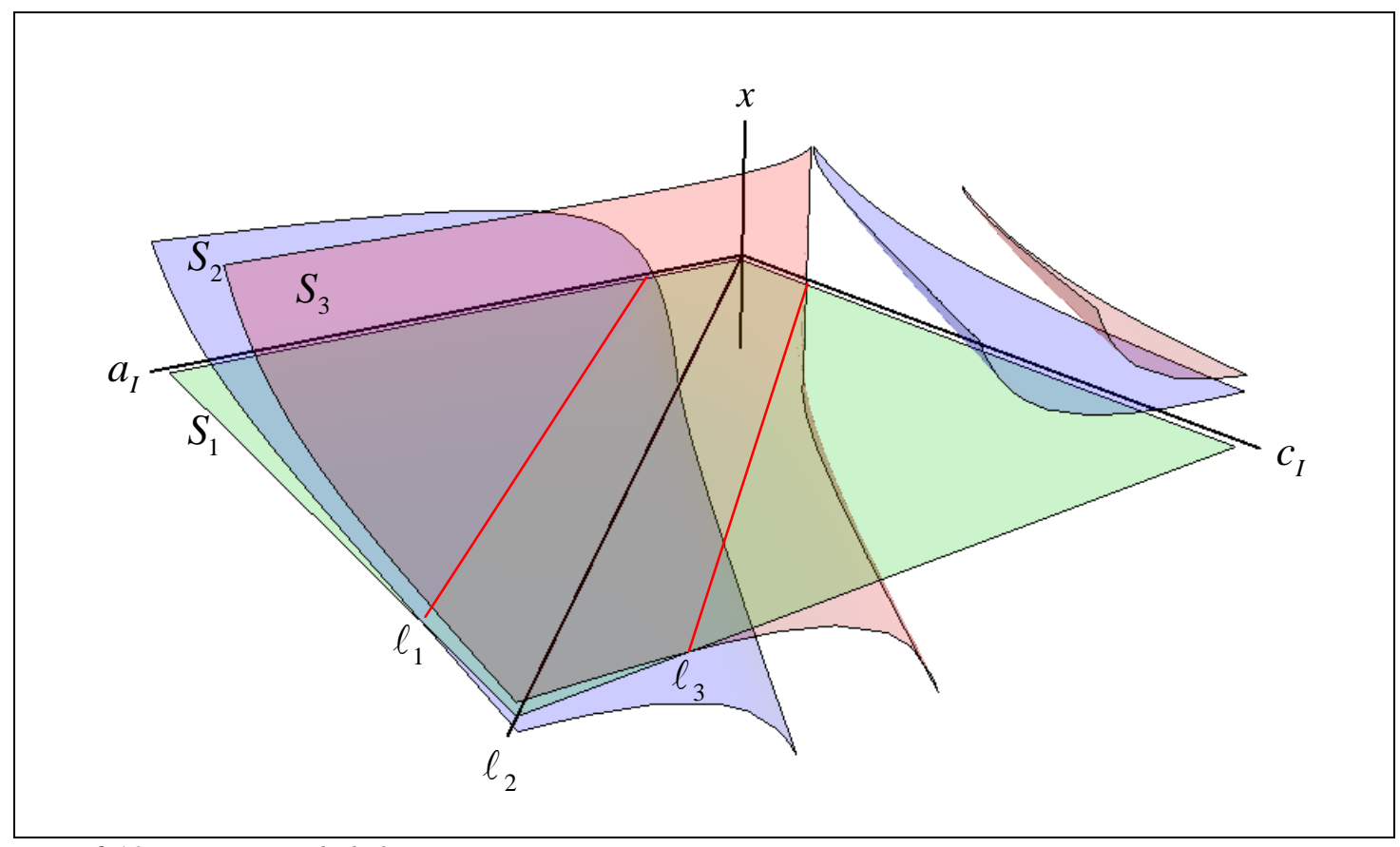

Fig.6.10: Diagrama de bifurcações para o ponto $P_{2,3}$. 
No diagrama da figura 6.10, o seguinte esquema de cores foi adotado:

- A superfície $S_{1}$ (esverdeada) representa os pontos $\mathrm{P}_{2}$ e $\mathrm{P}_{3}$;

- A superfície $S_{2}$ (azulada) representa o ponto $\mathrm{P}_{2 \mathrm{a}}$;

- A superfície $S_{3}$ (avermelhada) representa o ponto $\mathrm{P}_{3 \mathrm{a}}$;

- As linhas vermelhas $\ell_{1}$ e $\ell_{3}$ representam, respectivamente, os traços dos planos $\pi_{1}$ e $\pi_{3}$, ortogonais ao plano $a_{I} \times c_{I}$, tal que a expressão $\mu_{I}=\sqrt{\Omega^{2}+\frac{1}{\left(a_{I}-c_{I}\right)^{2}}}$ é satisfeita;

- A linha vermelha $\ell_{2}$ representa o traço do plano $\pi_{2}$, ortogonal ao plano $a_{I} \times c_{I}$, tal que a expressão $a_{I}=c_{I}$ é satisfeita.

Neste mesmo diagrama são discriminadas quatro regiões espaciais:

- Região R1 é aquela que contém todos os pontos do espaço $\left(x, a_{I}, c_{I}\right)$ que estão entre o plano $\pi_{1}$ e $c_{I}=0$;

- Região R2 1 é aquela que contém todos os pontos do espaço $\left(x, a_{I}, c_{I}\right)$ que estão entre o plano $\pi_{1}$ e $\pi_{2}$;

- Região $\mathrm{R} 2{ }_{3}$ é aquela que contém todos os pontos do espaço $\left(x, a_{I}, c_{I}\right)$ que estão entre o plano $\pi_{2}$ e $\pi_{3}$;

- Região R3 é aquela que contém todos os pontos do espaço $\left(x, a_{I}, c_{I}\right)$ que estão entre o plano $\pi_{3}$ e $a_{I}=0$;

A região $\mathrm{R} 1 \cup \mathrm{R} 3$ contém todos os pontos que satisfazem a condição $\sqrt{\Omega^{2}+\frac{1}{\left(a_{I}-c_{I}\right)^{2}}}>\mu_{I}$

A região $R 2_{1} \cup R 2_{3}$ contém todos os pontos que satisfazem a condição $\sqrt{\Omega^{2}+\frac{1}{\left(a_{I}-c_{I}\right)^{2}}}<\mu_{I}$.

A região $\mathrm{R} 1 \cup \mathrm{R} 22_{1}$ contém todos os pontos que satisfazem a condição $a_{I}>c_{I}$. 
A região $R 3 \cup R 2_{3}$ contém todos os pontos que satisfazem a condição $a_{I}<c_{I}$.

Para qualquer condição inicial $\left(x, a_{I}, c_{I}\right)$ que esteja na região $\mathrm{R} 3$, o sistema sempre evolui tendo a superfície S1 como atratora e a superfície S2 como repulsora (condição 6.10).

Para qualquer condição inicial $\left(x, a_{I}, c_{I}\right)$ que esteja na região $\mathrm{R} 2_{3}$, o sistema sempre evolui tendo a superfície S1 como atratora e a superfície S3 como repulsora (condição 6.12).

Para qualquer condição inicial $\left(x, a_{I}, c_{I}\right)$ que esteja na região $\mathrm{R} 2{ }_{1}$, o sistema sempre evolui tendo a superfície S1 como repulsora e a superfície S2 como atratora (condição 6.11).

Para qualquer condição inicial $\left(x, a_{I}, c_{I}\right)$ que esteja na região $\mathrm{R} 1$, o sistema sempre evolui tendo a superfície S1 como repulsora e a superfície S3 como atratora (condição 6.13).

Assim, quando $\Omega=\mu_{I}$, há somente o ponto de equilíbrio $\mathrm{P}$, assintoticamente estável para $a_{I}<c_{I}$. É importante perceber que as constantes do filtro $a_{I}$ e $c_{I}$ são ambas positivas e não-nulas. Quando $\Omega<\mu_{I}$, há, em princípio, dois pontos de equilíbrio, $\mathrm{P}_{2}$ e $\mathrm{P}_{3}$. Uma análise mais aprofundada revela a existência de outros dois pontos $\mathrm{P}_{2 \mathrm{a}}$ e $\mathrm{P}_{3 \mathrm{a}}$, próximos respectivamente a $\mathrm{P}_{2}$ e $\mathrm{P}_{3}$. Suas estabilidades estão dadas pelas condições expressas em (6.10), (6.11), (6.12) e (6.13), e dependem, em princípio, exclusivamente dos parâmetros $a_{I}, c_{I}, \mu_{I}$ e $\Omega$, todos positivos não-nulos. Mais uma vez, é importante ressaltar que os parâmetros $b_{I}$ e $b_{I I}$ dos filtros, respectivamente dos nós I e II, devem ser não-nulos, condição que foi assumida na obtenção do sistema de equações no espaço de fases. Ainda, a estabilidade destes PLLs não depende diretamente destes parâmetros.

\subsubsection{SINCRONISMO DE UM NÓ TIPO II}

No estudo do sincronismo de um nó tipo II, isto é, diretamente conectado a um nó tipo I, obteve-se nove pontos de equilíbrio: 
- $\Omega=\mu_{I}=\mu_{I I} \Rightarrow P=\left(+\frac{\pi}{2}, 0,0,-\pi, 0,0\right)$

- $\Omega=\mu_{I}<\mu_{I I} \Rightarrow P_{1,2}=\left(+\frac{\pi}{2}, 0,0,+\frac{\pi}{2}+\arccos \left[ \pm \sqrt{1-\left(\frac{\Omega}{\mu_{I I}}\right)^{2}}\right], 0,0\right)$

- $\Omega=\mu_{I I}<\mu_{I} \Rightarrow$

$$
\Rightarrow P_{3,4}=\left(\arccos \left[ \pm \sqrt{1-\left(\frac{\Omega}{\mu_{I}}\right)^{2}}\right], 0,0,+\frac{\pi}{2}+\arccos \left[ \pm \sqrt{1-\left(\frac{\Omega}{\mu_{I}}\right)^{2}}\right], 0,0\right)
$$

- $\Omega<\mu_{I}$ e $\Omega<\mu_{I I} \Rightarrow$

$$
\begin{aligned}
& \Rightarrow P_{5,6}=\left(\arccos \left[ \pm \sqrt{1-\left(\frac{\Omega}{\mu_{I}}\right)^{2}}\right], 0,0,\right. \\
& \left., \arccos \left[ \pm \sqrt{1-\left(\frac{\Omega}{\mu_{I}}\right)^{2}}\right]+\arccos \left[ \pm \sqrt{1-\left(\frac{\Omega}{\mu_{I I}}\right)^{2}}\right], 0,0\right) \\
& \Rightarrow P_{7,8}=\left[\arccos \left[ \pm \sqrt{1-\left(\frac{\Omega}{\mu_{I}}\right)^{2}}\right], 0,0,\right. \\
& \left., \arccos \left[ \pm \sqrt{1-\left(\frac{\Omega}{\mu_{I}}\right)^{2}}\right]+\arccos \left[\mp \sqrt{1-\left(\frac{\Omega}{\mu_{I I}}\right)^{2}}\right], 0,0\right)
\end{aligned}
$$

Os elucidativos diagramas de bifurcação relativos a estes nove pontos de equilíbrio não serão aqui apresentados uma vez que não foi possível simplificá-los adequadamente sem perda de generalidade. A dependência da estabilidade destes pontos em relação aos parâmetros já considerados será expressa apenas sob a forma de expressões matemáticas.

\subsubsection{ESTABILIDADE DO PONTO P}

O campo de vetores restrito à variedade central em torno de $\mathrm{P}$ é: 


$$
\left\{\begin{array}{l}
\dot{y}=\frac{\mu}{2}(y-z)\left\{\left(a_{I I}-c_{I I}\right) \mu \cdot y^{2}+2\left(c_{I I}-a_{I I}\right) z \cdot \mu \cdot y+y+z\left[\left(c_{I}-c_{I I}\right) z \cdot \mu-1\right]\right\} \\
\dot{z}=\frac{\mu}{2} z^{2}\left[\left(a_{I}-c_{I}\right) z \cdot \mu+1\right]
\end{array}\right.
$$

Em torno de $\mathrm{P}$, há outros quatro pontos de equilíbrio, $\mathrm{P}_{\mathrm{a}}, \mathrm{P}_{\mathrm{b}}, \mathrm{P}_{\mathrm{c}}$ e $\mathrm{P}_{\mathrm{d}}$ :

- $\mathrm{O}$ ponto $\mathrm{P}_{\mathrm{a}}$, de coordenadas $y=-\frac{1}{\left(a_{I}-c_{I}\right) \mu}$ e $z=-\frac{1}{\left(a_{I}-c_{I}\right) \mu}$, é assintoticamente estável para $\left(a_{I I}>a_{I} \wedge a_{I}<c_{I}<a_{I I}\right)$ e instável para $\left(a_{I I} \leq a_{I} \wedge c_{I} \neq a_{I}\right) \vee\left[a_{I I}>a_{I} \wedge\left(c_{I}<a_{I} \vee c_{I}>a_{I I}\right)\right] . \quad$ É importante ressaltar que estas condições são mais gerais que àquela obtida para o sincronismo do nó tipo $\mathrm{I}$, em (6.7). Para que o ponto $\mathrm{P}$ seja assintoticamente estável é necessário somente que $\left(a_{I}>c_{I}\right)$;

- O ponto $\mathrm{P}_{\mathrm{b}}$, de coordenadas $y=-\frac{1}{\left(a_{I I}-c_{I I}\right) \mu}$ e $z=0$, é instável para $\left(a_{I I}>c_{I I}\right)$. É interessante observar que este ponto é instável independentemente dos valores ou relações que possam assumir os parâmetros do nó tipo I. O diagrama de bifurcações correspondente ao ponto $\mathrm{P}_{\mathrm{b}}$ é idêntico, tanto para a variável $\mathrm{y}$, quanto para a variável $\mathrm{z}$, àquele da figura 6.9 .

- $\mathrm{O}$ ponto $\mathrm{P}_{\mathrm{c}}$, de coordenadas $y=-\frac{-a_{I}-2 a_{I I}+c_{I}+2 c_{I I}+\sqrt{a_{I}^{2}-2 c_{I} \cdot a_{I}+4 a_{I I}^{2}-4 a_{I I}\left(c_{I}+c_{I I}\right)+c_{I}\left(c_{I}+4 c_{I I}\right)}}{2\left(a_{I}-c_{I}\right)\left(a_{I I}-c_{I I}\right) \mu}$ e $z=-\frac{1}{\left(a_{I}-c_{I}\right) \mu}$, é assintoticamente estável para $\left(a_{I I}<a_{I} \wedge c_{I}>a_{I} \wedge c_{I I}>a_{I I}\right) \vee\left(a_{I I}=a_{I} \wedge c_{I}>a_{I I} \wedge c_{I I}>a_{I I}\right) \vee$ $\left(a_{I I}>a_{I} \wedge\left(\left(a_{I}<c_{I}<a_{I I} \wedge a_{I I}<c_{I I}<\frac{a_{I}^{2}-2 c_{I} \cdot a_{I}+4 a_{I I}^{2}+c_{I}^{2}-4 a_{I I} \cdot c_{I}}{4 a_{I I}-4 c_{I}}\right) \vee\right.\right.$ $\left.\left.\vee\left(c_{I} \geq a_{I I} \wedge c_{I I}>a_{I I}\right)\right)\right)$ e instável para 


$$
\begin{gathered}
\left(a_{I I}<a_{I} \wedge\left(c_{I}<a_{I} \vee\left(c_{I}>a_{I} \wedge \frac{a_{I}^{2}-2 c_{I} \cdot a_{I}+4 a_{I I}^{2}+c_{I}^{2}-4 a_{I I} \cdot c_{I}}{4 a_{I I}-4 c_{I}}<c_{I I}<a_{I I}\right)\right)\right) \vee \\
\vee\left(a_{I I}=a_{I} \wedge\left(c_{I}<a_{I I} \vee\left(c_{I}>a_{I I} \wedge \frac{a_{I}^{2}-2 c_{I} \cdot a_{I}+4 a_{I I}^{2}+c_{I}^{2}-4 a_{I I} \cdot c_{I}}{4 a_{I I}-4 c_{I}}<c_{I I}<a_{I I}\right)\right)\right) \vee \\
\vee\left(a_{I I}>a_{I} \wedge\left(c_{I}<a_{I} \vee\left(a_{I}<c_{I} \leq a_{I I} \wedge c_{I I}<a_{I I}\right) \vee\right.\right. \\
\left.\left.\quad \vee\left(c_{I}>a_{I I} \wedge \frac{a_{I}^{2}-2 c_{I} \cdot a_{I}+4 a_{I I}^{2}+c_{I}^{2}-4 a_{I I} \cdot c_{I}}{4 a_{I I}-4 c_{I}}<c_{I I}<a_{I I}\right)\right)\right) . \text { A mesma }
\end{gathered}
$$

observação que foi feita para o ponto $\mathrm{P}_{\mathrm{a}}$ também é válida para $\mathrm{P}_{\mathrm{c}}$;

- $O$ ponto $\mathrm{P}_{\mathrm{d}}$, de coordenadas

$$
\begin{gathered}
y=-\frac{a_{I}+2 a_{I I}-c_{I}-2 c_{I I}+\sqrt{a_{I}^{2}-2 c_{I} \cdot a_{I}+4 a_{I I}^{2}-4 a_{I I}\left(c_{I}+c_{I I}\right)+c_{I}\left(c_{I}+4 c_{I I}\right)}}{2\left(a_{I}-c_{I}\right)\left(a_{I I}-c_{I I}\right) \mu} \\
\text { e } z=-\frac{1}{\left(a_{I}-c_{I}\right) \mu}, \text { é assintoticamente estável para } \\
\left(a_{I I}<a_{I} \wedge c_{I}>a_{I} \wedge\left(\frac{a_{I}^{2}-2 c_{I} \cdot a_{I}+4 a_{I I}^{2}+c_{I}^{2}-4 a_{I I} \cdot c_{I}}{4 a_{I I}-4 c_{I}}<c_{I I}<a_{I I} \vee c_{I I}>a_{I I}\right)\right) \vee \\
\vee\left(a_{I I} \geq a_{I} \wedge c_{I}>a_{I I} \wedge\left(\frac{\left.\left.a_{I}^{2}-2 c_{I} \cdot a_{I}+4 a_{I I}^{2}+c_{I}^{2}-4 a_{I I} \cdot c_{I}<c_{I I}<a_{I I} \vee c_{I I}>a_{I I}\right)\right)}{4 a_{I I}-4 c_{I}}\right.\right. \\
\mathrm{e} \text { instável para } \\
\left(\left(( a _ { I I } < a _ { I } \wedge c _ { I } < a _ { I } ) \vee ( a _ { I I } = a _ { I } \wedge c _ { I } < a _ { I } ) \vee \left(a_{I I}>a_{I} \wedge\left(c_{I}<a_{I} \vee\left(a_{I}<c_{I}<a_{I I} \wedge\right.\right.\right.\right.\right. \\
\left.\left.\left.\left.\left.\wedge\left(c_{I I}<a_{I I} \vee a_{I I}<c_{I I}<\frac{a_{I}^{2}-2 c_{I} \cdot a_{I}+4 a_{I I}^{2}+c_{I}^{2}-4 a_{I I} \cdot c_{I}}{4 a_{I I}-4 c_{I}}\right)\right)\right)\right)\right)\right) . \text { As }
\end{gathered}
$$

mesmas observações que foram feitas para os pontos $\mathrm{P}_{\mathrm{a}}$ e $\mathrm{P}_{\mathrm{c}}$ também são válidas para $\mathrm{P}_{\mathrm{d}}$.

\subsubsection{ESTABILIDADE DOS PONTOS $P_{1,2}$}

$\mathrm{O}$ campo de vetores restrito à variedade central em torno de $\mathrm{P}_{1,2}$ é: 


$$
\left\{\begin{aligned}
\dot{y}= & \frac{\mu_{I}}{2}\left\{ \pm 2(y-z) \mu_{I}\left[c_{I I}+a_{I I}\left(c_{I I}(y-z) \mu_{I}-1\right)\right] k^{2}+\right. \\
& \left.+2(y-z)\left[a_{I I}(y-z) \mu_{I}+c_{I I} \cdot z \cdot \mu_{I}+1\right] k \pm z(y-z)\right\} \\
\dot{z}= & \frac{\mu_{I}}{2} z^{2}
\end{aligned}\right.
$$

Sendo $k \stackrel{\Delta}{=} \sqrt{\frac{\mu_{I I}^{2}}{\mu_{I}^{2}}-1}$.

Para $\left(c_{I I}<\frac{a_{I I} \cdot \sqrt{\mu_{I I}^{2}-\mu_{I}^{2}} \mp 1}{\sqrt{\mu_{I I}^{2}-\mu_{I}^{2}}}\right)$ o ponto $\mathrm{P}_{1,2}$ é instável. Em torno do ponto

$\mathrm{P}_{1,2}$ há outro ponto de equilíbrio, $\mathrm{P}_{1 \mathrm{a}, 2 \mathrm{a}}$, de coordenadas $y=\frac{a_{I I} \cdot k \cdot \mu_{I}-c_{I I} \cdot k \cdot \mu_{I} \mp 1}{a_{I I} \cdot \mu_{I}\left(c_{I I} \cdot k \cdot \mu_{I} \pm 1\right)} \mathrm{e}$ $z=0$, instável para $\left(c_{I I}>\frac{a_{I I} \cdot \sqrt{\mu_{I I}^{2}-\mu_{I}^{2}} \mp 1}{\sqrt{\mu_{I I}^{2}-\mu_{I}^{2}}}\right)$.

\subsubsection{ESTABILIDADE DOS PONTOS $P_{3,4}$}

$\mathrm{O}$ campo de vetores restrito à variedade central em torno de $\mathrm{P}_{3,4}$ é:

$\left\{\begin{array}{l}\dot{y}=-z \cdot \mu_{I I}\left[\left(a_{I I}-c_{I}\right) k \cdot \mu_{I I} \cdot y+y+a_{I I} \cdot k \cdot z \cdot \mu_{I I}\left(c_{I} \cdot k \cdot \mu_{I I}-1\right)\right] \\ \dot{z}=\mp k \cdot z \cdot \mu_{I I}\left[ \pm c_{I} \cdot k \cdot \mu_{I I}+a_{I}\left( \pm c_{I} \cdot z \cdot k \cdot \mu_{I I}+k \mp z\right) \mu_{I I} \mp 1\right]\end{array}\right.$

Sendo $k \stackrel{\Delta}{=\sqrt{\frac{\mu_{I}^{2}}{\mu_{I I}^{2}}-1}}$

Em torno de $\mathrm{P}_{3,4}$ há outros dois pontos de equilíbrio, $\mathrm{P}_{3 \mathrm{a}, 4 \mathrm{a}}$ e $\mathrm{P}_{3 \mathrm{~b}, 4 \mathrm{~b}}$ :

- $O$ ponto $\mathrm{P}_{3 \mathrm{a}, 4 \mathrm{a}}, y= \pm \frac{a_{I I} \cdot k\left(a_{I I} \cdot k \cdot \mu_{I I} \pm c_{I} \cdot k \cdot \mu_{I I} \mp 1\right)}{a_{I}\left(a_{I I} \cdot k \cdot \mu_{I I}-c_{I} \cdot k \cdot \mu_{I I}+1\right)} \mathrm{e}$ $z=\frac{\mp a_{I} \cdot k \cdot \mu_{I I}-c_{I} \cdot k \cdot \mu_{I I}+1}{a_{I} \cdot \mu_{I I}\left(c_{I} \cdot k \cdot \mu_{I I}-1\right)}$, não pode ser assintoticamente estável para nenhuma combinação de parâmetros; 
- $O$ ponto $\mathrm{P}_{3 \mathrm{~b}, 4 \mathrm{~b}} y= \pm \frac{a_{I I} \cdot k\left(a_{I I} \cdot k \cdot \mu_{I I} \pm c_{I} \cdot k \cdot \mu_{I I} \mp 1\right)}{a_{I}\left(a_{I I} \cdot k \cdot \mu_{I I}-c_{I} \cdot k \cdot \mu_{I I}+1\right)}$ e $z=0$, é instável para $c_{I}<\frac{1 \mp a_{I I} \cdot \sqrt{\mu_{I}^{2}-\mu_{I I}^{2}}}{\sqrt{\mu_{I}^{2}-\mu_{I I}^{2}}}$.

\subsubsection{ESTABILIDADE DOS PONTOS $P_{5,6}$}

$\mathrm{O}$ campo de vetores restrito à variedade central em torno de $\mathrm{P}_{5,6}$ é:

$$
\left\{\begin{aligned}
\dot{y}= & -z \cdot \Omega\left[a_{I I} \cdot \Omega \cdot k_{I I}^{2}+\left(-c_{I} \cdot k_{I} \cdot \Omega+a_{I I}\left(c_{I} \cdot z \cdot \Omega \cdot k_{I} \pm k_{I} \pm z\right) \Omega \mp 1\right) k_{I I}+\right. \\
& \left.+a_{I I} \cdot k_{I} \cdot z \cdot \Omega\left(c_{I} \cdot k_{I} \cdot \Omega \pm 1\right)\right] \\
\dot{z}=\mp & k_{I} \cdot z \cdot \Omega\left[ \pm c_{I} \cdot k_{I} \cdot \Omega+a_{I}\left(z+k_{I}\left( \pm c_{I} \cdot z \cdot \Omega \mp 1\right)\right) \Omega+1\right]
\end{aligned}\right.
$$

Sendo $k_{I} \stackrel{\Delta}{=} \sqrt{\frac{\mu_{I}^{2}}{\Omega^{2}}-1}$ e $k_{I I} \stackrel{\Delta}{=} \sqrt{\frac{\mu_{I I}^{2}}{\Omega^{2}}-1}$

De (6.23) se obtém $y=r, \forall r \in \mathbb{R}$ e $z=0$. Para

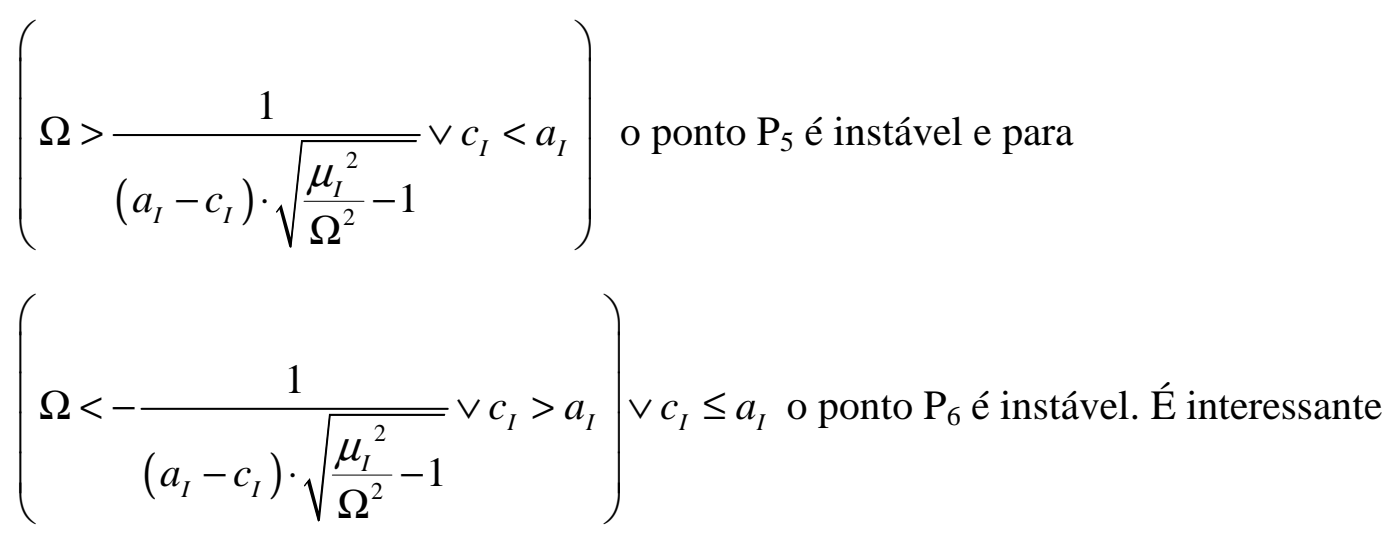
observar que os pontos $\mathrm{P}_{5}$ e $\mathrm{P}_{6}$ são instáveis independentemente dos valores ou relações que possam assumir os parâmetros do nó tipo II, dependendo somente daqueles relativos ao nó tipo I.

\subsubsection{ESTABILIDADE DOS PONTOS $\mathbf{P}_{7,8}$}

$\mathrm{O}$ campo de vetores restrito à variedade central em torno de $\mathrm{P}_{7,8}$ é: 


$$
\begin{aligned}
& \left\{\begin{aligned}
& \dot{y}=z \cdot \Omega\left[-a_{I I} \cdot \Omega \cdot k_{I I}^{2}+\left(-c_{I} \cdot k_{I} \cdot \Omega+a_{I I}\left(c_{I} \cdot z \cdot \Omega \cdot k_{I}+k_{I} \pm z\right) \Omega \mp 1\right) k_{I I} \mp\right. \\
&\left.\mp a_{I I} \cdot k_{I} \cdot z \cdot \Omega\left( \pm c_{I} \cdot k_{I} \cdot \Omega+1\right)\right] \\
& \dot{z}=-k_{I} \cdot z \cdot \Omega\left[c_{I} \cdot k_{I} \cdot \Omega+a_{I}\left( \pm z+k_{I}\left(c_{I} \cdot z \cdot \Omega \mp 1\right)\right) \Omega \pm 1\right]
\end{aligned}\right. \\
& \text { Sendo } k_{I} \stackrel{\Delta}{=} \sqrt{\frac{\mu_{I}^{2}}{\Omega^{2}}-1} \text { e } k_{I I}=\sqrt[\Delta]{\frac{\mu_{I I}^{2}}{\Omega^{2}}-1}
\end{aligned}
$$

De (6.24) se obtém $y=r, \forall r \in \mathbb{R}$ e $z=0$. Para

$$
\begin{aligned}
& \left(\Omega>\frac{1}{\left(a_{I}-c_{I}\right) \cdot \sqrt{\frac{\mu_{I}^{2}}{\Omega^{2}}-1}} \vee c_{I}<a_{I}\right) \text { o ponto } \mathrm{P}_{7} \text { é instável e para } \\
& \left(\Omega<-\frac{1}{\left(a_{I}-c_{I}\right) \cdot \sqrt{\frac{\mu_{I}^{2}}{\Omega^{2}}-1}} \vee c_{I}>a_{I}\right) \vee\left(c_{I} \leq a_{I}\right) \text { o ponto } \mathrm{P}_{8} \text { é instável. Mais uma vez, }
\end{aligned}
$$

é interessante observar que os pontos $\mathrm{P}_{7}$ e $\mathrm{P}_{8}$ são instáveis independentemente dos valores ou relações que possam assumir os parâmetros do nó tipo II, dependendo somente daqueles relativos ao nó tipo I.

No próximo capítulo, este texto se encerra com a contraposição entre os resultados esperados e obtidos, todas as conclusões pertinentes ao trabalho de pesquisa realizado, bem como as expectativas ou sugestões para a sua continuidade. 


\section{CAPÍTULO VII}

\section{CONCLUSÕES}

Neste trabalho foi possível determinar, via Teoria da Variedade Central e Teoria de Bifurcações, valores qualitativos ou relações entre os parâmetros de uma rede OWMS de PLLs de $3^{\mathrm{a}}$ ordem que proporcionem a existência e a estabilidade do estado síncrono. Para tanto, foram introduzidos dois sinais de excitação no oscilador mestre de grande ocorrência na prática, o degrau e a rampa de fase. As equações assim obtidas não são simples de se resolver analiticamente, mesmo com o auxílio de softwares específicos, como o Mathematica 6.

No caso de uma rede com apenas três osciladores, um mestre e dois escravos em cadeia simples, a descrição da dinâmica da rede pode depender de até 6 parâmetros, algebricamente relacionados por desigualdades. Quase todas foram determinadas, com algumas exceções em que somente foi possível predizer faixas de parâmetros para que o ponto de equilíbrio fosse instável. Provavelmente, seria necessário que se procedesse a uma aproximação de maior ordem, seja das funções trigonométricas em série de Taylor, seja nos polinômios estimadores do Teorema da Variedade Central. Seria interessante se os resultados aqui obtidos pudessem ser verificados na prática.

A grande expectativa de todo pesquisador é que o fruto do seu trabalho possa, no mínimo, contribuir com o trabalho de outro pesquisador. Caso isso ocorra, provavelmente será na mesma área de conhecimento de seu estudo. Seguindo essa linha de pensamento, espera-se que este texto tenha contribuído de alguma forma, para a Teoria de Sistemas Dinâmicos e para o desenvolvimento da Teoria do Sincronismo, dentro da Engenharia de Telecomunicações e Controle. 


\section{REFERÊNCIAS BIBLIOGRÁFICAS}

BANERJEE, D.. PLL Performance, Simulation and Design Hanbook. 4th Ed. Dog Ear Publishing, 2006.

BEAR, M. F.; CONNORS, B. W.; PARADISO, M. A.. Neurociências: desvendando o sistema nervoso. $2^{\mathrm{a}}$. ed.. Porto Alegre: Artmed, 2002.

BENNATON, J. F.; PIQUEIRA, J. R. C.; O teorema de Hopf, acoplado ao método do Lugar das Raízes, na Análise de Malhas de Sincronismo. In: X CONGRESSO NACIONAL DE MATEMÁTICA APLICADA E COMPUTACIONAL - 59/65, Gramado - R. S., setembro de 1987.

BEST, R. E., Phase-Locked Loops: Design, Simulation and Applications. $5^{\text {th }}$ ed.. New York: McGraw-Hill, 2003.

CARR, J. Applications of Centre Manifold Theory. New York: SpringerVerlag, 1981.

DIRETRIZES PARA APRESENTAÇÃO DE DISSERTAÇÕES E TESES. Serviço de Bibliotecas da Escola Politécnica da Universidade de São Paulo. São Paulo, 2001.

FIEDLER - FERRARA, N.; PRADO, C. P. C. DO. Caos: uma Introdução. $2^{\mathrm{a}}$. ed.. São Paulo: Editora Edgard Blücher LTDA, 1995.

GARCIA, P. A.. Redes Simples de Malhas de Sincronismo de Fase: uma análise via Teoria de Sistemas Dinâmicos. Dissertação (Mestrado). Engenharia Elétrica da Universidade Presbiteriana Mackenzie. São Paulo, 2000. 
GARDNER, F. M.. Phase Lock Techniques. New York: John Willey \& Sons, Inc., 1979.

GREEN, D. N.. Lock-In, Tracking and Acquisition of ACG - Aided PhaseLocked Loops. IEEE Transactions on Circuits and Systems. Vol. CAS-32, nº, dezembro, 1983.

GUCKENHEIMER, J.; HOLMES, P.J.. Nonlinear Oscillations, Dynamical Systems, and Bifucation of Vector Fields. New York: Springer-Verlag, 1983.

KROUPA, V. F.. Frequency Synthesis: Theory, Design et Applications. New York: J. Wiley, 1973.

LINDSEY, W. C.; GHAZVIVIAN, F.; HAGMANN, W. C.; DESSOUKY, K.. Network Syncronization. Proceedings of IEEE, vol. 73, no 10, 1445 - 1467, Outubro, 1985.

LINDSEY, W. C.. Syncronization Systems in Communication and Control. Prentice-Hall, Englewood Cliffs, New Jersey, 1972.

LINDSEY, W. C.; CHALK, M. C. Phase-Locked Loops, IEEE Press, New York, 1986.

MARGARIS, N. I. Non-linear Analog Phase Locked Loop. New York: Springer-Verlag, 2004.

MARMO, C. N.. Sincronismo em Redes Mestre-Escravo de Via Única: Cadeia Simples, Estrela Simples e Mista. Dissertação (Mestrado). Escola Politécnica, Universidade de São Paulo. São Paulo, 2003. 
MARMO, C. N.; FARIA, W. F. DE; PIQUEIRA, J. R. C.; MONTEIRO, L. H. A.. Sincronismo em Redes Mestre - Escravo: Comparação de Topologias. In: XIV CONGRESSO BRASILEIRO DE AUTOMÁTICA, Natal - RN, setembro de 2002 .

MARQUES, N.; MENNA - BARRETO, L. (orgs.). Cronobiologia: Princípios e Aplicações. São Paulo: Editora da Universidade de São Paulo, 1997.

MARTHA, L. A.; BRASELTON, J. P.. Differential Equations with Mathematica. Elsevier Academic Press 2004

MONTEIRO, L. H. A.. Sistemas Dinâmicos. São Paulo: Editora Livraria da Física, 2002.

MONTEIRO, L. H. A.; SANTOS, R. V.; PIQUEIRA, J. R. C.. Estimating the Critical Number of Slave Nodes in a Single Chain PLL Network. IEEE Communications Letters, Vol. 7. No. 9, September 2003.

MONTEIRO, L. H. A.; FAVARETTO, D. N; PIQUEIRA, J. R. C.. Bifurcation analysis for third-order phase-locked loops, IEEE Signal Process. Lett. 2004; 11(5):494-6.

OGATA, K.. Engenharia de Controle Moderno. $3^{\mathrm{a}}$. ed.. Rio de Janeiro: Editora Prentice - Hall do Brasil LTDA, 1998.

ORSATTI, F. M.. Redes Mutuamente Sincronizadas de DPLLs. Modelagem, Simulação e Otimização. Tese (Doutoramento). Escola Politécnica, Universidade de São Paulo. São Paulo, 2008.

PIQUEIRA, J. R. C.. Aplicação da teoria qualitativa de equações diferenciais a problemas de sincronismo de fase. Tese (Doutoramento). Escola Politécnica, Universidade de São Paulo. São Paulo, 1987. 
PIQUEIRA, J. R. C.. Uma contribuição ao estudo das redes com malhas de sincronismo de fase. Tese (Livre - Docência). Escola Politécnica, Universidade de São Paulo. São Paulo, 1997.

PIQUEIRA, J. R. C.; CASTILlO-VARGAS, S. A.; MONTEIRO, L. H. A.. Two-way master-slave double-chain networks: limitations imposed by linearmaster drift for second order PLLs as slave nodes. IEEE Communications Letters, vol. 9, no. 9, pp. 829-831, 2005.

PIQUEIRA, J. R. C.; MARMO, C. N.; MONTEIRO, L. H. A.. Using Central Manifold Theorem in the Analisys of Master-Slave Synchronization Networks. Journal of Communications and Networks, vol. 6, no. 3, pp. 197-201, 2004.

PIQUEIRA, J. R. C.; ORSATTI, F. M.; MONTEIRO, L. H. A.. Computing with Phase Locked Loops: choosing gains and delays. IEEE Transactions on Neural Networks, vol.14, n⿳0 1, janeiro, 2003.

SHAO, G.; BERMAN, F.; WOLSKI, R.. Master/slave computing on the grid. Proceedings of IEEE - 21st COMPSAC, 2000.

SOHAIL, S.; RAJ, G.. Replication of multimedia data using master-slave Architecture. Proceedings of IEEE - 21st COMPSAC, 1997.

VITERBI, A. J.. Principles of Coherent Communication. New York: MC Graw - Hill, 1966.

WIGGINS, S.. Introduction to Applied Nonlinear Dynamical Systems and Chaos. New York: Springer-Verlag, 1990. 